\title{
A revision of Nearctic Dasysyrphus (Diptera: Syrphidae)
}

\author{
Michelle Mary Locke
}

A thesis submitted to the Faculty of Graduate Studies and Research in partial fulfillment of the requirements for the degree of

Master of Science

in

Biology

Carleton University

Ottawa, Ontario

(C) 2012

Michelle Mary Locke 
Library and Archives

Canada

Published Heritage

Branch

395 Wellington Street

Ottawa ON K1A ON4

Canada
Bibliothèque et

Archives Canada

Direction du

Patrimoine de l'édition

395 , rue Wellington

Ottawa ON K1A ON4

Canada
Your file Votre référence

ISBN: 978-0-494-91543-1

Our file Notre référence

ISBN: 978-0-494-91543-1
NOTICE:

The author has granted a nonexclusive license allowing Library and Archives Canada to reproduce, publish, archive, preserve, conserve, communicate to the public by telecommunication or on the Internet, loan, distrbute and sell theses worldwide, for commercial or noncommercial purposes, in microform, paper, electronic and/or any other formats.

The author retains copyright ownership and moral rights in this thesis. Neither the thesis nor substantial extracts from it may be printed or otherwise reproduced without the author's permission.
AVIS:

L'auteur a accordé une licence non exclusive permettant à la Bibliothèque et Archives Canada de reproduire, publier, archiver, sauvegarder, conserver, transmettre au public par télécommunication ou par l'Internet, prêter, distribuer et vendre des thèses partout dans le monde, à des fins commerciales ou autres, sur support microforme, papier, électronique et/ou autres formats.

L'auteur conserve la propriété du droit d'auteur et des droits moraux qui protege cette thèse. $\mathrm{Ni}$ la thèse ni des extraits substantiels de celle-ci ne doivent être imprimés ou autrement reproduits sans son autorisation.
In compliance with the Canadian Privacy Act some supporting forms may have been removed from this thesis.

While these forms may be included in the document page count, their removal does not represent any loss of content from the thesis.
Conformément à la loi canadienne sur la protection de la vie privée, quelques formulaires secondaires ont été enlevés de cette thèse.

Bien que ces formulaires aient inclus dans la pagination, il n'y aura aucun contenu manquant. 
Abstract

Dasysyrphus Enderlein (Diptera: Syrphidae) has posed taxonomic challenges to researchers in the past, due to their low interspecific variation. In the present study, DNA data (mitochondrial cytochrome oxidase c subunit I) were combined with morphology to help sort out species concepts. This led to two species being resurrected from synonymy (D. laticaudus and D. pacificus) and the discovery of one new species (D. occidualis sp. nov.). An additional new species was described based on morphology alone (D. richardi sp. nov.), as the specimens were too old to obtain COI. Part of the taxonomic challenge presented by this group arises from missing type specimens. Neotypes are designated here for D. pauxillus and D. pinastri to bring stability to these names. An illustrated key to 13 Nearctic species is presented, along with descriptions, maps and supplementary data. A phylogeny based on COI is also presented and discussed. 


\section{Acknowledgements}

Funding support was provided by grants to J.H. Skevington (NSERCCANPOLIN, NSERC Discovery, and Agriculture and Agri-Food Canada) and scholarships to me (Carleton University Faculty of Graduate Studies and Research Travel Bursaries).

Thank you to J.H. Skevington and Agriculture and Agri-Food Canada for providing me with the resources and equipment required to complete this project and to the Biodiversity Institute on Ontario for DNA barcoding support.

Thank you to the insect collection managers who graciously provided me with specimens for study. Also to E. Proctor, P. Laska, T. Neilsen, W. van Steenis, M. Hauser for specimens.

Thanks to S. Kelso for his guidance in the molecular lab, to W. Knee for his help with the Bayesian analysis, to A. Young for putting up with me during all of our field work (and making me drive up a treacherous mountain to find a rare fly!), to D. Doczkal for advice on Dasysyrphus.

I would like to thank Vladimir Davydov for the beautiful photograph of the Dasysyrphus larva and A. Young for the photograph of the adult (Figure 1).

I would like to give a special thank you my parents, Dave and Karen, whose love and support have helped make this possible. Also, my sister, Stephanie, brother Sean, and best friends, Emily and Heather for their words of encouragement, which always put a smile on my face. Thank you. 
Table of Contents

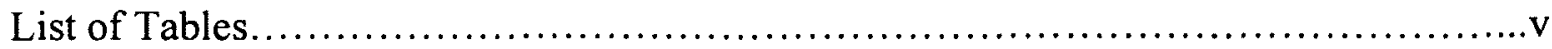

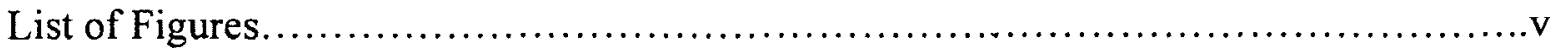

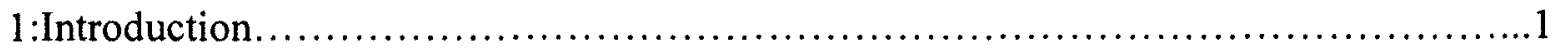

1.1: Species Concepts................................................

1.2: What is Dasysyrphus?...............................................

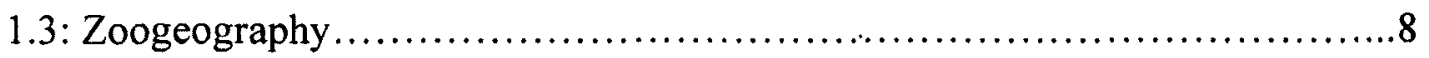

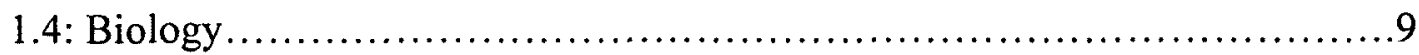

1.5: History of Dasysyrphus Taxonomy....................................11

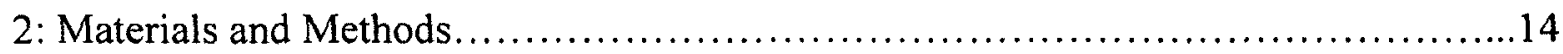

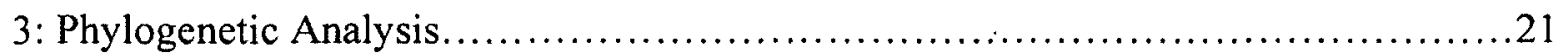

3.1 Results of Analyses................................................ 22

3.2 Discussion of Analyses........................................... 24

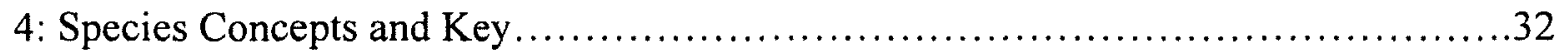

4.1 Generic Redescription............................................. 32

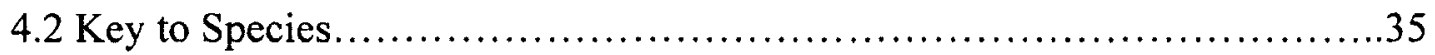

4.3 Species Descriptions............................................ 39

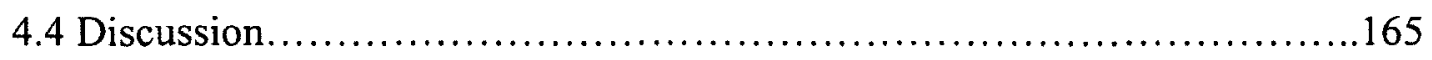

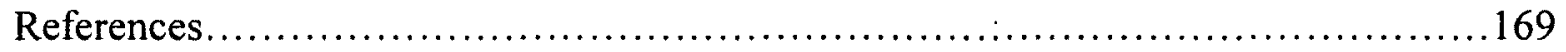

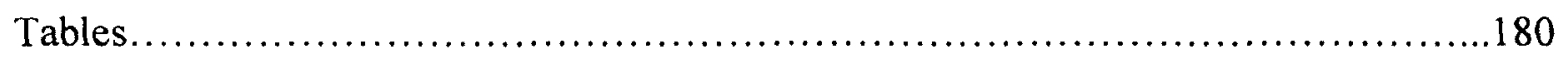

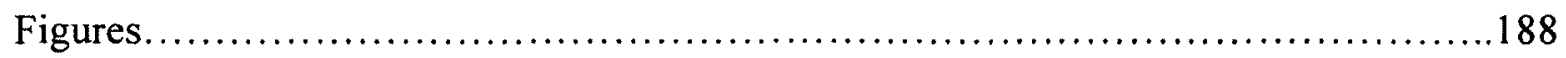




\section{List of Tables}

Table 1: Voucher information for molecular Dasysyrphus specimens................180

Table 2: Intraspecific variation and nearest neighbour values......................187

\section{List of Figures}

Fig. 1: Dasysyrphus larva and adult field photographs.......................... 188

Fig. 2: Semi-strict consensus tree from Parsimony analysis........................ 189

Fig. 3: Majority rule consensus tree from Bayesian analysis..................... 190

Fig. 4: Neighbour-Joining tree............................................... 191

Fig. 5: Dasysyrphus amalopis and D. creper dorsal and lateral habitus.................195

Fig. 6: Dasysyrphus intrudens complex dorsal and lateral habitus.................. 196

Fig. 7: Dasysyrphus nigricornis and laticaudus dorsal and lateral habitus.............197

Fig. 8: Dasysyrphus venustus and D. limatus dorsal and lateral habitus...............198

Fig. 9: Dasysyrphus lotus and richardi dorsal and lateral habitus.....................199

Fig. 10: Dasysyrphus occidualis and D. pacificus dorsal and lateral habitus............200

Fig. 11: Dasysyrphus pauxillus and D. pinastri dorsal and lateral habitus.............201

Fig. 12: Dasysyrphus amalopis and D. occidualis lateral genitalia....................202

Fig. 13: Dasysyrphus creper and D. limatus lateral genitalia......................203

Fig. 14: Dasysyrphus lotus and D. nigricornis lateral genitalia....................204

Fig. 15: Dasysyrphus laticaudus and D. pacificus lateral genitalia..................205

Fig. 16: Dasysyrphus pauxillus and D. pinastri lateral genitalia.....................206

Fig. 17: Dasysyrphus intrudens complex and D. venustus lateral genitalia...........207

Fig. 18: Dasysyrphus amalopis and D. creper range map.........................208 
Fig. 19: Dasysyrphus intrudens complex range map............................209

Fig. 20: Dasysyrphus laticaudus and D. richardi range map........................210

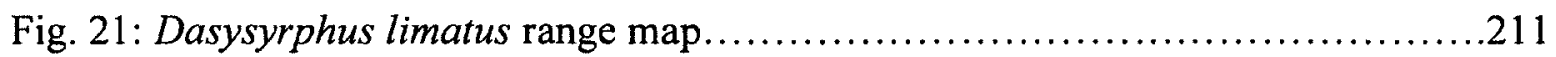

Fig. 22: Dasysyrphus lotus and D. nigricornis range map.........................212

Fig. 23: Dasysyrphus occidualis range map.....................................213

Fig. 24: Dasysyrphus pacificus range map......................................2 214

Fig. 25: Dasysyrphus pauxillus and D. pinastri range map........................215

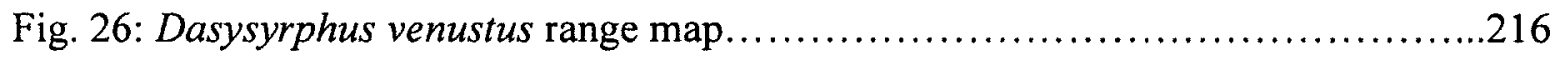


Chapter 1: Introduction

\section{1: Species Concepts}

The concept of a species is something that is widely debated in the scientific community, and has been for years. "No one definition has satisfied all naturalists; yet every naturalist knows vaguely what he means when he speaks of a species" (Darwin 1859). It is becoming more widely understood that biologists actually share a common concept of species. They can regularly discuss species without referencing the concept of species, and typically disagreement over a species does not usually arise unless specific species concepts are referred to in the discussion (Hey 2006). Over the years many species concepts have been proposed, some more widely accepted than others, and there is no consensus as to which one should be used (Hey 2006; De Queiroz 2007). The reason this matter is of such great debate is that the species is the fundamental unit in biology and has been likened to an atom in the field of physics (Du Rietz 1930; Mayr 1942; De Queiroz 2005). The species is the unit of study for many biologists, including systematists, ecologists, behaviourists, physiologists, evolutionary biologists, palaeontologists, geneticists and molecular biologists (De Queiroz 2005). Many scientific studies are based on what someone has identified as a species.

The most broadly recognized concept of species comes from Ernst Mayr (1942). He defined his Biological Species Concept (BSC) as "groups of actually or 
potentially interbreeding populations, which are reproductively isolated from other such groups". While widely used, there are many times when this definition is sometimes impossible and often impractical to apply. It does not work in the case of asexually reproducing organisms or sexual organisms that hybridize, as many plants and animals do (Simpson 1951). It is also not feasible to apply this concept when studying fossils or museum specimens that include little to no ecological data.

In insect taxonomy, we rarely have data to delimit species based on concepts that require ecological and/or biological data. Hennig (1966) recognized that the BSC did not account for the evolution of species through time and reproductive barriers could not exist as one species evolved from another so he proposed the Phylogenetic Species Concept (PCS). Over time this concept has been gradually refined and is currently defined as "...the smallest aggregation of (sexual) populations or (asexual) lineages diagnosable by a unique combination of character states" (Wheeler \& Platnick 2000). There is no subdivision of species into subspecies, therefore the PSC diagnoses more species than the BSC, which allows for subspecies (Dillon \& Fjeldså 2005).

Many other concepts have been put forth throughout the years (see Table 1 in De Queiroz 2007), each providing new and different criteria for delimiting species. The reason so many concepts exist is due to the fact that there are so many disciplines in biology (De Queiroz 2007). Niches are important to an ecologist, genes to a geneticist and diagnosable morphological characters to some 
taxonomists. One can expect each field to value the criteria it studies, placing it as the most significant tool in species diagnoses and delimitation (De Queiroz 2007). The problem with this is that some of these concepts can delimit different numbers of species (for example, in Dillon \& Fjeldså 2005). So how do we reconcile this? What approach do we use when we are fortunate enough to have multiple data sets?

De Queiroz (2007) has proposed an idea that removes the ambiguity of the species concept, bringing us closer to a global concept. He (2007) states that the way to solve the species problem is to separate the concept of a species from the ways in which a species is delimited. All of the current species concepts define a species as an independently evolving metapopulation lineage, whether explicitly stated or not (De Queiroz 2007). This means that a population is following one evolutionary path, so when a subpopulation breaks off and heads down another evolutionary path it then has become a new species. This is what De Queiroz (2007) defines as the primary defining property of a species and is what should be the only necessary property of a species. He says that to delimit species one can use the secondary defining properties, which are the other criteria given by alternate species concepts. These are such criteria as reproductive isolation as stated by the BSC; occupation of different niches, as in the Ecological Species Concept (ESC); monophyly, as in the PSC and so forth. Since multiple selection pressures can act on populations to produce new species, the order in which the secondary criteria arise is always different (De Queiroz 2007). Depending on what point species are at in 
their evolution, the current concepts could have different outcomes, predicting one species or two. However, if we use these secondary criteria just to delimit a species rather than define it, any one of these will be valid (De Queiroz 2007). As long as at least one of these criteria exist then we can interpret this as evidence for multiple species and the more evidence you have, the more convinced one will be that, in fact, there are two lineages with independently evolving trajectories (De Queiroz 2007). This total evidence approach now shifts that debate away from the definition of a species to how to delimit them (De Queiroz 2007).

A total evidence approach is quite practical in taxonomy. The main data sets used by most taxonomists are morphological and molecular characters. These are used primarily for practical reasons. Most taxonomists are working in collections that contain specimens that can vary in age from a few to over a hundred years old. Since they have specimens at their disposal, morphology is an easily obtainable data set. With DNA sequencing becoming faster, easier and cheaper it is also quite practical for most taxonomists to use a fragment of their study organism's DNA as another data set. The major drawback to this is that it is difficult to obtain DNA from the older specimens. Putative species distribution is often informative as most species follow predictable patterns. These data are easily obtained from specimen labels. Ecological and biological data can also be used, if available. Ecological data may be obtained directly from label data when recorded, or inferred by harvesting data layers (climate, elevation, vegetation, etc.) from geocoded locality data. Biological data may be obtained by observing pairs of specimens that were collected 
while in copula (usually pinned together or somehow associated). Literature can also be helpful in obtaining some of this data that has been reported in ecological studies, surveys and catalogues. All of this information is valuable in delimiting species, if available.

A revision of Nearctic Dasysyrphus Enderlein 1938 is needed to sort out species boundaries in this genus. In the past, the interpretation of morphology has produced incongruent species concepts, largely due to perceived intraspecific variation and low interspecific variation. A total evidence approach is used here to help delimit species and to facilitate a better understanding of Dasysyrphus.

\section{2: What is Dasysyrphus?}

Fluke (1933) was the first author to define what would later be called Dasysyrphus and he provided higher level and species keys with descriptions of all Nearctic species. He defined this group as the amalopis group under the genus Metasyrphus Matsumura (in Matsumura and Adachi), 1917. He stated that he was not prepared to associate his four distinct groups of Metasyrphus with genera due to the confusion of some of the generic concepts in the Old World and lack of material from the Old World to compare his specimens to. His four groups are still considered monophyletic lineages and are now known as Dasysyrphus (his amalopis group), Epistrophe Walker 1852 (his emarginatus group), Eupeodes (Lapposyrphus)

Dusek \& Laska 1967 (his lapponicus group) and Eupeodes (Metasyrphus) (his 
Metasyrphus s.s). Note that both the subgenera Epistrophe and Epistrophella Dusek \& Laska, 1967 were included in the emarginatus group by Fluke.

Dasysyrphus was erected as a subgenus of Syrphus Fabricius, 1775 in 1938 by Günther Enderlein (1938). Enderlein distinguished Dasysyrphus from the rest of the Syrphus group by the conspicuously dense hair on its eyes. Dasysyrphus is similar to many other genera in the subfamily Syrphinae, having yellow spots or stripes on a black abdomen, apparently mimicing Hymenoptera. Enderlein included 16 species in his new genus and described two more from the Palaearctic, Oriental and Afrotropical regions, although all seven species from the Oriental and Afrotropical regions and three from the Palaearctic have now been placed in other genera. Enderlein did not include any Nearctic species in Dasysyrphus. The Nearctic species were first moved into Dasysyrphus in 1965 when Stone et al. published their "A Catalog of the Diptera of America North of Mexico" (Stone et al. 1965). They referenced the amalopis group of Fluke (1933) for the species that they included in Dasysyrphus. Vockeroth (1992) provided a key to the Canadian and Alaskan species of Dasysyrphus and species descriptions. No other recent revisionary work has been done.

The first Dasysyrphus species to be described was Musca pinastri De Geer, 1776, from Europe. De Geer provided a description of the larvae and adults of this species and provided some ecological observations as well. De Geer was also one of the few authors to provide illustrations of the specimens he was describing. Forty- 
one additional species were described before Enderlein defined Dasysyrphus in 1938 and twenty-eight species have been erected since (including current synonyms; Pape \& Thompson 2010a). Previous to Dasysyrphus being erected, species of this genus were often included in Syrphus, Musca Linnaeus, 1758 and Scaeva Fabricius, 1805 (Pape \& Thompson 2010a). Of the species occurring in the Nearctic, Musca pinastri was the first to be described. In the Nearctic it is known to exist only in Greenland (Vockeroth 1992). Next, Syrphus venustus Meigen, 1822 was described from Europe. In 1875, Osten Sacken described Syrphus amalopis from the White Mountains in New Hampshire, making it the first species, of what is now Dasysyrphus, to be described from a specimen originating in the Nearctic region. Two years later Osten Sacken described Syrphus intrudens Osten Sacken, 1877 from California. Ten years later Williston described Syrphus lotus Williston, 1887 and Syrphus pauxillus Williston, 1887 from Arizona and New Mexico, respectively. Williston also included a key to the species of Syrphus in his paper. In 1895 Snow described two more species, Syrphus creper from Colorado and New Mexico, and Syrphus disgregus, which is currently considered a synonym of $D$. venustus, from New Mexico. Lovett then described Syrphus pacifica (in Cole \& Lovett), 1919, a synonym of pauxillus, from Oregon. The species Syrphus reflectipennis Curran, 1921, was then described from Ontario. Syrphus limatus Hine, 1922 was next described from Alaska. Curran (1925) provided descriptions for three more species: Syrphus laticaudus, Syrphus osburni and Syrphus laticaudatus. The former two species were described from Ontario, the latter from British Columbia. 


\section{3: Zoogeography}

Dasysyrphus is mainly a holarctic genus. Thirty-six of the 43 currently recognized species are known to occur in North America, Europe and Asia, with eight species recorded from the Oriental region and one species recorded from the Neotropical region (some species occur in more than one region; Pape \& Thompson 2010a). Vockeroth (1969) states that this is a typical distribution for a genus in the tribe Syrphini. Syrphini include many genera with low intrageneric diversity in the Old World and North America, and relatively few genera with high intrageneric diversity in South America (Vockeroth 1969). Vockeroth postulates that two forms of Syrphini entered South America in the Tertiary and diversified to fill different niches, while many forms existed in North America and the Old World to fill those same niches, allowing for less diversity to develop within a genus. He suggests that the forms that entered South America, did so before South America was isolated so had plenty of time to diversify. Syrphini fauna of the Old World and North America had frequent connections in the Bering Strait, allowing their fauna to develop essentially as a single unit (Vockeroth 1969). When North and South America became permanently connected in the Pliocene there was an interchange of a few species. This may explain why a genus like Dasysyrphus has a fairly low intrageneric diversity and why there are so many more species in the Holarctic than in the Neotropical region. Its absence from the Afrotropical region is not explained, although Vockeroth (1969) points out that among Syrphini of this region, the generic patterns are similar to those of the Nearctic. So perhaps the reason 
Dasysyrphus have not thrived in the Afrotropical region stems from other constraints, perhaps ecological in nature.

\section{4: Biology}

Little is known about the biology of Dasysyrphus. The majority of biological information we have comes from studies done in Europe. No larvae are known from endemic Nearctic species and as a result only adult ecology can be discussed. Much of what is known from the Nearctic and what will be discussed in this study has been gathered from label data from specimens.

Larvae are mottled, resemble bark and are easily recognized by a pair of long tapering projections on the anal segment, which help them to blend into their surroundings (Fig. 1A; Rotheray \& Gilbert 2011; Rotheray 1993). They are known to be arboreal in both coniferous and deciduous trees (Rotheray 1993). Larvae are predatory and feed mainly on homopterans, but records of them feeding on other insects have been noted. Rojo et al. (2003) provide a catalogue to Dasysyrphus species and their larval prey. Their review of the literature suggests that the larvae feed mainly on Aphididae, but have been found feeding on Adelgidae, Diptera larvae, Neuroptera, Lepidoptera, Hymenoptera and Coleoptera. Rojo et al. (2003) also recorded the plant associations of these Dasysyrphus larvae and their prey. Most studies found these larvae associated with trees of the families Pinaceae, Aceraceae, Ulmaceae, Fagaceae and Rosaceae. Some studies reported finding 
Dasysyrphus larvae on woody shrubs in the families Fabaceae, Rosaceae and Celastraceae and a few other studies reported finding them on herbaceous plants in the families Brassicaceae and Chenopodiaceae (Rojo et al. 2003). Goeldlin de Tiefenau (1974) reported that larvae he reared in the lab wrapped themselves around a stem and remained in one position to feed until ready to pupate. This position allowed them to block the path and intercept prey as they passed by.

Adults (Fig. 1B) are believed to be important pollinators. Their specific pollination habits are unknown but they are commonly found on flowers on sunny days. Most of the museum specimens I have observed have pollen grains stuck to their body hairs and in their gut. Adults have been recorded as visiting flowers of deciduous trees and smaller herbaceous plants (see Speight 2011). In the Nearctic region, specimens have been recorded as being collected from Prunus virginiana Linnaeus (Williston 1887) and Geranium Linnaeus (Snow 1895). I believe that Dasysyrphus are generalist pollinators. While Dasysyrphus has been an understudied genus, a review of pollination in syrphids by Rotheray and Gilbert (2011) shows that the genera that have been studied are effective generalist pollinators. New data on flower visitation (from labels) is included in the species accounts below. They are imperfect mimics, not exactly resembling any specific hymenopteran, but having the black and yellow colouration common to many Hymenoptera. Rotheray and Gilbert (2011) and Penney et al. (2012) provide overviews and discuss some hypotheses as to why this imperfect mimicry may exist in syrphids. Since Dasysyrphus larvae tend to be arboreal, the adults are typically found in or near 
forests. Speight (2011) reports in his European species accounts that the following species are found in mainly coniferous, but sometimes deciduous forests: albostriatus, eggeri, friuliensis, hilaris, lenensis, pauxillus, pinastri, postclaviger, tricinctus and venustus. Dasysyrphus nigricornis is reported as being found in taiga (Neilsen 1998) and dwarf-shrub tundra, although much confusion exists over the name, so the full extent of its range and habitat are not known (Speight 2011).

\section{5: History of Dasysyrphus Taxonomy}

Dasysyrphus is a poorly understood group taxonomically. Like many other genera in the tribe Syrphini it shows a narrow range of variation in characters among species, but has a very distinctive habitus, so that Dasysyrphus is easily recognizable from other genera (Vockeroth 1969). This similarity in appearance among species has made defining species based on morphology difficult. There is no consensus as to which characters are variable and which are important in species characterization, which has led to very different species concepts. Due to the difficulty in using morphological characters and defining species concepts, creating a diagnostic key to this group has been difficult. Dasysyrphus has never been treated on a worldwide scale. For the Nearctic, Fluke (1933) created his key to America north of Mexico (Canada and the USA), which treated 11 species. Vockeroth (1992) then created a key for his monograph on the Syrphinae of Canada, Alaska and Greenland. In this key he synonymized some of the species Fluke had treated, leaving a total of seven species (six that Fluke had treated, plus pinastri, which was 
not included by Fluke). While Vockeroth's key does not formally treat the USA he does discuss the full extent of all of the species ranges, and he does discuss species occurring in the contiguous USA, but not in Canada. Useful keys to other regions include: Bartsch et al. (2009) for Nordic countries, Barkalov (2007) for the Asian region of Russia, Huo et al. (2005) for China, van Veen (2004) for Northwestern Europe, Stubbs \& Falk (2002) for Britain, Ghorpade (1994) for India and Peck (1974) for the Palaearctic. A manuscript for the Neotropical region is currently being written by Thompson (pers. comm.), which is why only the Nearctic, as opposed to the New World, is revised here.

The majority of the problems with Dasysyrphus lie within the venustus and the pauxillus groups. With some authors defining new, very similar taxa, and others synonymizing them, they all used different criteria to define species, splitting them and lumping them again. Since many of the species concepts are relatively old they do not include an in-depth description of characters or illustrations. As well, these complexes have been treated as Holarctic, with no in-depth studies done to compare Nearctic and Palaearctic specimens. Laska \& Bicik (1996) tried to solve a piece of the venustus complex in the Palaearctic. There was an argument as to whether $D$. venustus and $D$. hilaris (Zetterstedt, 1842) were the same species. Laska and Bicik hypothesized that they are in fact two species, however there are several intermediate specimens that may be hybrids, as both species occur in the same localities. Alternatively, the 'intermediates' may simply be additional cryptic 
species. It is typically believed now that these are two different species, but in the past, they were grouped as one, creating confusion among authors.

In the Nearctic, the most recent concept of venustus (Vockeroth 1992) has typically included all of the specimens with abdominal maculae crossing the abdominal margin, excluding limatus, for which the species concepts are well understood. Within this broad venustus concept there is extremely high variation in the pattern of the maculae, much more so than in any other species of Dasysyrphus. These maculae range from almost straight, with little medial constriction to lunulate and deeply constricted. With this much variation it is likely that multiple species exist. Within the pauxillus group in the Nearctic there have been two other names proposed, but currently they are both synonymized under pauxillus. Furthermore, the name pauxillus has been used throughout the Palaearctic to describe species occurring there. There are many Palaearctic species that have a similar habitus to pauxillus and their concepts are even more confused. This group needs to be carefully examined on a worldwide scale to fully understand the species boundaries.

As an independent source of information, molecular data may help solve some of these issues. Molecular data have not been used before to attempt to untangle the species concepts of Nearctic Dasysyrphus. It has the potential to shed new light on these concepts and provide a new set of information to lend support to species concepts within Dasysyrphus. These data should also help to bridge the gap 
between Old World and New World research. Ongoing and unpublished morphological work by Doczkal (pers. comm.) and genetic work by Ståhls (pers. comm.) on the Palaearctic venustus group has illustrated new characters, and some new species. Doczkal has discovered subtle new characters and character systems that have never been applied to Dasysyrphus.species before. His research was corroborated by Ståhls who, sequenced COI (mitochondrial cytochrome oxidase c subunit I) and ITS2 (nuclear internal transcribed spacer region 2) for all of his putative species. Despite being unpublished, this Palaearctic research has helped form a ground plan for tackling the Nearctic species. Molecular data will also give us our first picture of the evolutionary relationships within Dasysyrphus.

With so much uncertainty surrounding Nearctic Dasysyrphus it needs to be studied in depth to attempt to find new characters, both morphological and molecular, and to produce new, more solid concepts of how many species exist in the Nearctic. The following revision attempts to describe each species in detail and paint a clearer picture of the boundaries of each species.

Chapter 2: Materials and Methods

Specimens for this project were obtained from the following collections (arranged in alphabetical order by codon): Biodiversity Institute of Ontario, (BIOUG), Guelph, ON; California Academy of Sciences (CAS), San Francisco, CA; Canadian National Collection of Insects, Arachnids and Nematodes (CNC), Ottawa, 
ON; California State Collection of Arthropods (CSCA), Sacramento, CA; C.P. Gillette Museum of Arthropod Diversity, Colorado State University (CSUC), Fort Collins, C0; University of Guelph Insect Collection (DEBU), Guelph, ON; Essig Museum of Entomology (EMEC), University of California Berkeley, Berkeley, CA; Utah State University Insect Collection (EMUS), Logan, UT; Field Museum of Natural History (FMNH), Chicago, IL; Instituto Nacional de Biodiversidad (INBio), Santo Domingo de Heredia, Costa Rica; J.B. Wallis Museum of Entomology, University of Manitoba (JBWM), Winnipeg, MB; Lyman Entomological Museum, McGill University (LEMQ), Ste. Anne de Bellevue, QC; Museum of Comparative Zoology, Harvard University (MCZ), Cambridge, MA; Martin Hauser personal collection (MHPC); Albert J. Cook Arthropod Research Collection, Michigan State University (MSUC), East Lansing, MI; Oregon State University (OSAC), Corvallis, OR; C.A. Triplehorn Insect Collection, Ohio State University (OSU), Columbus, $\mathrm{OH}$; Royal British Columbia Museum (RBCM), Victoria, BC; Royal Ontario Museum (ROME), Toronto, ON; Snow Entomological Museum, University of Kansas (SEMC), Lawrence, KS; Simon Fraser University (SFUC), Burnaby, BC; E.H. Strickland Entomological Museum, University of Alberta (UASM), Edmonton, AB; Spencer Museum, University of British Columbia (UBCZ), Vancouver, BC; United States National Museum (USNM), Washington, DC; Eleanor Proctor Personal Collection (donated to $\mathrm{CNC}$ ). Abbreviations for the collections are taken from Evenhuis (2012). Each specimen was given a unique identifying number (if one was not present from its home institution) and the label information was entered into the CNC's specimen database. 
To obtain DNA barcodes ( 658 base pairs from the $5^{\prime}$ end of cytochrome c oxidase subunit 1), single legs were removed from selected specimens and were sent to the Biodiversity Institute of Ontario in Guelph, ON. There they extracted, amplified, purified and sequenced the DNA following protocols given by Hajibabaei et al. (2005). Some COI sequences were obtained at the Agriculture \& Agri-Food Canada, Eastern Cereal and Oilseed Research Centre Core Sequencing Facility (Ottawa, ON, Canada) (protocols below). DNA was extracted using a DNeasy® Tissue kit (Qiagen Inc., Santa Clara, CA, USA), using their Animal Tissue SpinColumn Protocol. Originally whole legs were used, however better results were obtained when the leg was ground up using the TissurRuptor (Qiagen Inc., Santa Clara, CA, USA).

COI was amplified in $25 \mu \mathrm{L}$ reactions with $2.5 \mu \mathrm{L} 10 \mathrm{X}$ ExTaq PCR buffer, $0.65 \mu \mathrm{L} 25 \mathrm{mM} \mathrm{MgCl}_{2}, 1 \mu \mathrm{L}$ of each $10 \mu \mathrm{M}$ primer (one forward and one reverse), $2 \mu \mathrm{L}$ $10 \mu \mathrm{M}$ dNTPs, $13.7 \mu \mathrm{L} \mathrm{H}_{2} \mathrm{O}, 0.15 \mu \mathrm{L}$ ExTaq HS DNA polymerase (Takara Bio USA, Madison, WI, USA), and $4 \mu \mathrm{L}$ genomic DNA template. Different primers were used in an ongoing attempt to yield better results. The primers used were: LCO1490 (hebF; LC01490 5'-GGTCAACAAATCATAAAGATATTGG-3'; (Folmer et al. 1994)), COI-Dipt1858F (5'-GGNTGRCANGTNTAYCC-3'; (Gibson et al. 2011)), HCO2198 (hebR; 5'TAAACTTCAGGGTGACCAAAAAATCA-3'; (Folmer et al. 1994)), K699R (5'GGGGGTAAACTGTTCATCC-3'; Wahlberg 2010) and COI-Dipt-2183R (5'CCAAAAAATCARAATARRTGYTG-3'; (Gibson ê al. 2011)). PCR amplification was performed on an Eppendorf ep Gradient S Mastercycler (Eppendorf AG, Hamburg, 
Germany). The amplification cycle used was $94^{\circ} \mathrm{C}$ for $3 \mathrm{~min} . ; 40 \times\left(94^{\circ} \mathrm{C}\right.$ for $1 \mathrm{~min}$.; $52^{\circ} \mathrm{C}$ for $1 \mathrm{~min} . ; 2^{\circ} \mathrm{C}$ for $1.5 \mathrm{~min}$.); $72^{\circ} \mathrm{C}$ for $5 \mathrm{~min}$. The PCR amplification products were run through $1 \%$ agarose electrophoresis gels for visual confirmation of PCR success. The PCR products were then purified in preparation for sequencing using ExoSAP-IT@ protocol (USB Corp., Cleveland, OH, USA) or an E-Gel@ Agarose Gel Electrophoresis System (Invitrogen ${ }^{\mathrm{TM}}$, Carlsbad, CA, USA) according to the protocol described in Gibson et al. (2010). Sequencing reactions were conducted using an ABI BigDye $®$ Terminator v3.1 Cycle Sequencing kit (PE Applied Biosystems, Foster City, CA, USA). The reactions took place in a volume of $10 \mu \mathrm{L}$, consisting of $1.5 \mu \mathrm{L}$ BigDye ${ }^{8}$ Terminator v1.1/3.1 Sequencing Buffer (5X), $1 \mu \mathrm{L}$ of Ready Reaction Mix (2.5X), $0.5 \mu \mathrm{L} 10 \mu \mathrm{M}$ primer, $2 \mu \mathrm{L} \mathrm{H} \mathrm{H}_{2} \mathrm{O}$ and $5 \mu \mathrm{L}$ purified PCR product. Sequencing of the COI purified PCR product was performed at the Agriculture \& Agri-Food Canada, Eastern Cereal and Oilseed Research Centre Core Sequencing Facility (Ottawa, ON, Canada). Sequencing reactions were purified using the ABI ethanol/EDTA/sodium acetate precipitation protocol and analysed on an ABI 3130xl Genetic Analyzer (PE Applied Biosystems, Foster City, CA, USA). The resulting sequence chromatograms were edited and formed into contigs using Sequencher 4.7 (Gene Codes Corp., Ann Arbor, MI, USA). The contigs were examined in Mesquite version 2.73 (build 544; Maddison \& Maddison 2010) and aligned manually by the translated amino acid codons.

Specimens submitted to the Barcoding Institute of Ontario were chosen for sampling based on age. Specimens up to 40 years old were originally used; 
however, many of the older specimens did not yield any DNA. Specimens up to 10 years old were then used with a higher success rate. In all, $\sim 60 \%$ of the specimens yielded some sequence data. Sequencing performed by the author was done to attempt to target sequences from species that were not obtained previously, to gain complete sequences for species where only partial sequences were previously obtained and to gain sequences from newly obtained specimens. Specimens up to 50 years old were used, however younger specimens (10 years or younger) typically yielded DNA and specimens older than that were less reliable. In total, COI sequences were obtained for 20 species of Dasysyrphus.

To initially analyze the COI sequence data a neighbour-joining tree was created from 354 sequences (specimens listed in Table 1) using the Barcode of Life Data Systems (Ratnasingham \& Hebert 2007). The neighbour-joining method was used to identify COI sequences that clustered together in the tree. The specimens whose COI sequences made up these clusters were then studied under the microscope to determine if morphological characters existed to separate species. External morphological characters and male genitalic characters were examined. Male genitalia were removed from the specimen, cleared in hot $95 \%$ lactic acid, and mounted in glycerol to be observed. For storage, the genitalia were placed in glycerol in genitalia vials and placed on the original specimen's pin, below the specimen. The molecular data were important in settling the controversy over which characters were variable and which were useful species level synapomorphies. New and often subtle characters were also discovered that may 
have been lost in the morass of perceived variation during previous work on the genus.

A data matrix consisting of 42 specimens with $658 \mathrm{COI}$ characters each (bases, $A, T, C$ or $G$ ) was analyzed more rigorously. Of the 354 total sequences, one sequence that best represented each putative species was chosen; a second sequence was used if a large amount of intraspecific variation appeared on the NJ tree. Thirty-two Dasysyrphus sequences were combined with ten outgroup taxa. Outgroup taxa were chosen from the subfamily Syrphinae. The species Meligramma guttata (Fallén, 1817), M. triangulifera (Zetterstedt, 1843) and Fagisyrphus cinctus (Fallén, 1817) were used because they were found to be sister to Dasysyrphus (Mengual et al. 2008). The remaining seven specimens were chosen due to their hypothesized close relation to Dasysyrphus (Mengual et al. 2008). They are from the genera Scaeva, Eupeodes and Melangyna. A Parsimony analysis was conducted using PAUP* 4.0 (Swofford 2003). The outgroup was set to be paraphyletic. A heuristic search was launched, using step-wise addition and 1000 reps. Jackknife supports (JKS) were calculated, excluding $36 \%$ of the characters (as recommended by Farris et al. (1996)) and running 100 replicates. Bremer supports (BRS) were generated using TreeRot v3 (Sorenson and Franzosa 2007) and PAUP* 4.0 (Swofford 2003) using a heuristic search.

Bayesian analysis was conducted using MrBayes 3.2.1 (Ronquist et al. 2011), which was submitted remotely to run at the CIPRES Science Gateway V3.1 (Miller et 
al. 2010). The best-fit model of molecular evolution was determined using MrModeltest v2.3 (Nylander 2004). This model was determined to be GTR+I+G. In MrBayes a Markov Chain Monte Carlo (MCMC) method was used. The settings were as follows: two independent runs, with nucmodel $=4$ by $4, N_{s t}=6$, rates $=$ invgamma, samplefreq $=1000$, four chains $=$ one cold and three hot. The COI dataset was run for 5 million generations with a burn-in of 1000. A majority-rule consensus tree displaying the posterior probability supports for each node was generated in Mesquite. This was generated using the remaining trees, excluding the burn-in.

Terminology for morphological characters follows Thompson (1999) and Cumming \& Wood (2009) for the terms not outlined in Thompson's glossary. Terminology for the male genitalia follows Cumming \& Wood (2009). In the species descriptions the majority of the distribution and ecological data were taken from the CNC's specimen database, which contains the label data from all of the specimens loaned to the author by the institutions listed above. Primary type material is listed before the material examined list and it is indicated in brackets if it was examined or not. For types examined, the label data is listed using " " to indicate each label and / to separate each line of that label. Other information, including sex and deposition is stated here as well. Deposition codons used follow Evenhuis (2012). For type material not examined, the information presented was compiled from the literature. The material examined lists present the paratypes examined, followed by all other specimens examined, sorted by country, state/province and latitude. All plant names used in the ecology sections of the species descriptions 
were verified and updated to current generic and specific concepts through VASCAN (Brouillet et al. 2010) and the USDA PLANTS Database (USDA 2012). For research into the etymology of the scientific names the work by Brown (1956) was used. Range maps were created using SimpleMappr (Shorthouse 2010). Images for figures were taken on a Leica DM550B compound microscope (Leica Microsystems Inc., Concord, ON) and a Canon EOS 50D camera equipped with a $100 \mathrm{~mm}$ macro lens (Canon Canada Inc., Mississauga, ON). Zerene Stacker (Zerene Systems LLC, Richland, WA) was used to create the montage images.

Colour is an important character in many syrphids. It is useful in identifying genera and species. However, it has been shown that colour in Syrphidae can be temperature dependent. In an experiment by Dusek \& Laska (1974), it was shown that low pupal development temperatures result in darker adults and higher pupal development temperatures result in lighter adults. Preservation techniques and age of the specimen can also affect its colour. Colour characters must therefore be used with great caution and one should recognize that there is high probability for variation in these characters within a species. Specimens of Dasysyrphus typically have two colours, yellow and black. In some specimens the yellow has darkened, leaving areas on the specimen looking more brown. In other specimens, the black pigment has faded, causing areas to appear lighter, more brown, than they would on a live specimen. The following key and descriptions use the terms "light" and "dark", as opposed to specifically describing colours seen on specimens. Generally "dark" refers to black and "light" refers to yellow; however, because both colours can look 
brown and could possibly be described as black or yellow depending on the observer, the author has chosen to use "light" and "dark" to try to help remove ambiguity.

Chapter 3: Phylogenetic Analysis

This is the first quantitative phylogeny, molecular or morphological, proposed for Dasysyrphus. Based on morphology, Dusek \& Laska (1967) assigned subgenera and Vockeroth (1969) placed species into species groups. Analysis of the phylogeny based on COI data and a comparison of these results with these previous taxonomic proposals is discussed below.

\subsection{Results of Analyses}

A semi-strict consensus tree was generated from 26 most parsimonious trees. The length of the best tree was 505 steps. The Consistency Index (CI) excluding uninformative characters was 0.4141 . The Retention Index (RI) was 0.6826. For the Jackknife analysis, 143 characters were parsimony-informative. A Jackknife 50\% Majority Rule consensus tree was generated. Jackknife and Bremer support values were mapped onto the semi-strict consensus tree (Fig. 2). A majority-rule consensus tree was generated from 8002 trees from the Bayesian analysis (Fig. 3). 
Both Bayesian and Parsimony analyses support the contention that Dasysyrphus is monophyletic (Figs 2 \& 3). In the Bayesian analysis this is well supported with a Posterior Probability (BPP) of 1.0. Support from the parsimony analysis is weak with Jackknife support (JKS) of 63 and Bremer support (BRS) of 2 . The backbone of the Dasysyrphus phylogeny is poorly supported. The Bayesian analysis generates a weakly supported polytomy, with few well-supported clades. The semi-strict consensus tree is more resolved, but weakly supported at the deeper nodes.

Dasysyrphus limatus and tricinctus form a well-supported lineage (BPP = 1.0; $J K S=100 ; B R S=12$ ) with unique genitalia characters that support the hypothesis (see discussion below). However, placement of this clade is different in both analyses. There is weak support for both its placement as sister to the intrudens complex (Fig. 2) and as sister to all remaining species of Dasysyrphus (Fig. 3). The clade consisting of albostriatus and eggeri is another with high support (BPP = 1.0 ; $\mathrm{JKS}=98 ; \mathrm{BRS}=5$ ). This clade is also supported by unique genitalic synapomorphies (see discussion below). It too, is resolved in different positions in both trees. In the Bayesian analysis it sister to the clade of lotus, Dasysyrphus sp. 23 (from South Korea), lenensis, amalopis, pinastri, occidualis, nigricornis, creper, pacificus, laticaudus and pauxillus (BPP $=0.9955 ;$ Fig. 3 ) and in the parsimony analysis it is weakly supported as sister to all of the other Dasysyrphus (JKS =63; BRS = 2; Fig. 2). 
The clade of lotus, Dasysyrphus sp. 23 (from South Korea), lenensis, amalopis, pinastri, occidualis, nigricornis, creper, pacificus, laticaudus and pauxillus is well supported in both trees $(\mathrm{BPP}=0.9483 ; \mathrm{JKS}=82 ; \mathrm{BRS}=3$ ). Both analyses show similar relationships among species within the clade, with the parsimony analysis being slightly more resolved (Fig. 2).

Dasysyrphus sp. 19 (from Costa Rica) and bilineatus are poorly supported sister species in the parsimony analysis (Fig. 2) and both are part of the unresolved polytomy backbone of Dasysyrphus in the Bayesian analysis (Fig. 3).

The intrudens species complex is well supported with a BPP = 1 in the Bayesian analysis; intrudens sp. $1-10$ are strongly supported (JKS $=85$; $\mathrm{BRS}=3$ ) as a clade with intrudens sp. 11 weakly supported as sister to this group in the parsimony analysis (Fig. 2). The Bayesian analysis shows strong support for venustus being sister to the intrudens species complex (BPP =0.9699; Fig. 3), but venustus is sister to bilineatus $+s p .19+$ the intrudens group in the parsimony analysis (Fig. 2).

\subsection{Discussion of Analyses}

In 1967, Dusek \& Laska put forth subgeneric concepts for Dasysyrphus. They proposed Dasysyrphus s.s. for albostriatus and eggeri, Syrphella Goffe for tricinctus and Dendrosyrphus Dusek \& Laska for friuliensis, hilaris, lunulatus and venustus. 
Vockeroth (Vockeroth 1969) treated Syrphella as a synonym of Dasysyrphus for practical reasons (see below). In 1988, Peck treated Dendrosyrphus as a synonym of Dasysyrphus (Peck 1988), without justification. The subgenera proposed by Dusek \& Laska (1967) have been treated as synonyms ever since.

The other concept put forth to organize the species of Dasysyrphus into taxonomic groupings was put forth by Vockeroth (1969). He proposed using four species groups based on genitalia form, but did not go so far as to use subgeneric names. Vockeroth did not use subgeneric names because "the absence of definitive external characters makes this course impractical" (Vockeroth 1969). Vockeroth's species groups are: the albostriatus group, including albostriatus and eggeri (Schiner, 1861); the lunulatus Meigen, 1822 group, including laticaudus, lotus, lunulatus, pacificus, pauxillus and Dasysyrphus sp. (from Brazil); the arcuatus (Fallén 1817) group, including amalopis, arcuatus, bilineatus (Matsumura \& Adachi 1917), hilaris (Zetterstedt, 1843), koningsbergeri (Meijere, 1914), osburni, laticaudatus, reflectipennis and Dasysyrphus sp. (from New Guinea); and the tricinctus (Fallén 1817) group, including lotus and tricinctus. Of note: the name arcuatus was suppressed (ICZN 1972) as the name was in a state of confusion and venustus was used instead, as its concept was more stable (Dusek \& Laska 1969). The name lunulatus was in a confused state and had been used for both venustus (of authors) and pinastri (of authors; Vockeroth 1986). Vockeroth (1969) used the name as pinastri; however, its correct usage should be as a synonym of venustus (Vockeroth 1986). The name amalopis was also in a confused state having been used as 
amalopis s.s, occidualis $\mathbf{s p}$. nov. and as a species in the intrudens complex in the literature. Vockeroth treats amalopis incorrectly as a member of the intrudens complex. Lastly, lotus is treated twice in two different species groups by Vockeroth (1969). Both the Bayesian and Parsimony analyses presented here (Figs. 3 \& 2 , respectively) suggest that lotus is sister to pinastri and its allies and I believe Vockeroth was correct in placing it in his lunulatus group. His placement of lotus in the tricinctus group is odd, as his groupings are based mainly on genitalia and the genitalia of lotus are very different from that of tricinctus. I believe that the name lotus under the tricinctus group is a typo and that the name limatus was intended here. This would make much more sense as the genitalia and external morphological characters discussed by Vockeroth make sense if looking at limatus, but not lotus.

Based on the phylogeny proposed here, Vockeroth's species groups (with a few nomenclatural updates, as discussed), based on genitalia are quite accurate. The subgenera proposed by Dusek and Laska are also quite accurate, however another subgenus would need to be erected for the pinastri group to maintain monophyly. I choose to follow Vockeroth and agree that species groups are warranted and are useful in discussing natural groups within Dasysyrphus, but that differences between these groups are not great enough to warrant subgenera within this genus. I support the continued synonymy of subgenera with Dasysyrphus. 
Based on evidence from the Bayesian and Parsimony analyses and the material I have seen I propose the following modifications to Vockeroth's species groups:

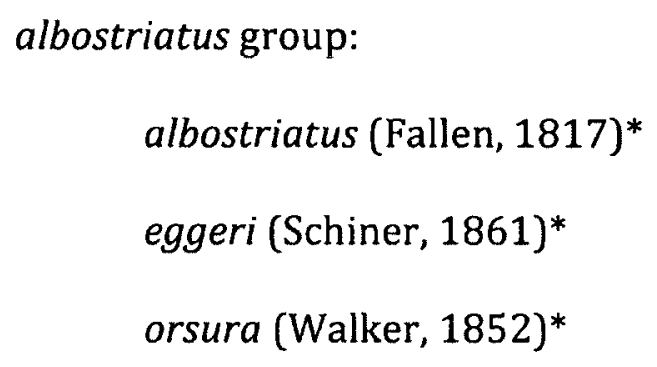

tricinctus group: 
limatus (Hine, 1922)*

tricinctus (Fallen, 1817)*

venustus group (formerly arcuatus group):

sp. 19 (from Costa Rica)*

bilineatus (Matsumura, 1917)*

friuliensis (Goot, 1960)*

hilaris (Zetterstedt, 1843)*

intrudens species complex (sensu Locke \& Skevington, this paper)*

koningsbergeri (Meijere, 1914)

postclaviger (Stys \& Moucha, 1962)

venustus (Meigen, 1822)*

* - species seen by author

Both the concepts by Dusek \& Laska and Vockeroth have highlighted $D$. tricinctus as a unique species. Genitalia characters given by Vockeroth (1969), mainly the shape and enlargement of the basiphallus and the short distiphallus (Fig. 13B), point to tricinctus and limatus being of close relation, and the Bayesian and Parsimony analyses agree. However, their placement as sister to the intrudens complex is not supported by genitalic synapomorphies that characterize the venustus group. Despite a lack of morphological evidence to place these species as sister to all Dasysyrphus, this strong evidence against their inclusion in the venustus 
group suggests that the Bayesian placement of these taxa is preferred over the parsimony analysis.

The albostriatus group is distinctive in both external morphology and genitalia (Vockeroth 1969). The long, slim distiphallus, without its apical end flared outwards, in a horn-like shape, and the distinct pollinose stripes on the scutum are particularly characteristic of this group. It was distinguished from other Dasysyrphus by both Dusek \& Laska (1967) and Vockeroth (1969). The COI evidence provided here also agrees with the concepts put forward by these authors. Their relationship to other Dasysyrphus is not clear from these analyses, although stronger support is given to its placement as sister to the pinastri group. There is some morphological evidence to back this up; the shape of the surstyli is similar between these two groups, as they are triangular in lateral view and flattened posteriorly.

The pinastri group (formerly the lunulatus group of Vockeroth (1969)) is well supported by the DNA evidence presented here. This group has variations within the shape of its gonostyli, basiphallus and distiphallus, but some similarities in the habitus of these structures occur that reflect the relation of this group. The gonostyli range from claw- (Fig. 14A) to boot-shaped (Fig. 16A), but the apical ends all taper to a point, and are very dissimilar to the rest of Dasysyrphus. The basiphallus usually has spines (Figs 14B, 15A, 15B, 16A, 16B) or pile (Fig. 13A) towards the apex, whereas it is smooth in the other Dasysyrphus (Fig. 14A). The 
basiphallus is faucet-shaped (Figs 13A, 14A, 14B, 15A, 15B, 16A, 16B) as in other Dasysyrphus, except for the tricinctus group. The pinastri group distiphallus is elongate, sometime bent basally and sometimes with a ventral projection. The apical end is flared out into a horn, both dorsally and ventrally, which is mostly sclerotized but membranous at the apex, usually with setulae dorsally (Figs 13A, 14A, 14B, 15A, 15B, 16A, 16B). Other Dasysyrphus either have a very shortened distiphallus (the tricinctus group), no apical horn (the albostriatus group) or the horn flares out on the dorsal edge considerably more than the ventral edge and the dorsal side is covered in setae (the venustus group).

Lastly is the venustus group (the arcuatus group of Vockeroth (1969) and Dendrosyrphus of Dusek \& Laska (1967)). This group is not recovered as monophyletic by either of the analyses presented here; however, they are of close relation in the trees presented. The Bayesian analysis has the majority of the species resolved in a clade, with two species (D. sp. 19 (of Costa Rica) and bilineatus) coming out as a polytomy with the rest of the group (Fig. 3). The parsimony analysis renders this group paraphyletic, with the tricinctus group in the middle (Fig. 2). The species listed in this group have similar genitalic characters. The shape of the gonostyli is similar throughout (Figs 17A, 17B), although it is similar to that of the tricinctus and albostriatus groups as well. The distiphallus shape is unique to the group, in that it is elongate and flared out into a horn at the apex, but the end is flared more dorsally and the dorsal side is covered in setae (Fig. Figs 17A, 17B). 
More data are required to test the monophyly of this group, however it is a convenient grouping to discuss such morphological characters.

While genitalia characters provide much insight into the relationships, a look should be taken at one other character commonly used to distinguish Dasysyrphus species. This is the pattern of the abdominal maculae; more specifically, if those maculae on tergites $3 \& 4$ cross the margin of the abdomen or not. In the tricinctus and venustus groups they cross the margin (Figs $6 \& 8$ ) and in the albostriatus and pinastri groups they do not (Figs $5,7,9,10,11$ ). The Parsimony analysis shows not crossing the margin as the ancestral state, with crossing the margin evolving once (Fig. 2), while the Bayesian analysis shows the opposite, that crossing the margin is the ancestral state and that not crossing the margin evolved once (Fig. 3). Either is plausible, although as discussed above, more merit is placed on the Bayesian results. It is hard to imagine why this character is of such significance and is not prone to homoplasy in the group. Both analyses support the high value that has been placed on this character in both past and present efforts to distinguish species or species groups.

This phylogeny supports Vockeroth's zoogeography hypotheses (Vockeroth 1969). There are no clear lineages dividing the Palaearctic and Nearctic, suggesting that there was regular and repeated contact of the fauna in these regions, thus no clear pattern of radiation in either region. With only a few Neotropical species in existence, it is conceivable that they diversified after arriving from the north once 
these two regions were connected (Vockeroth 1969). It is difficult to make too many comments on this matter, as this phylogeny is highly biased towards Nearctic taxa. More evidence needs to be gathered to paint a more accurate picture of how all of the species of Dasysyrphus are related and have evolved through time. The addition of more gene regions should add more depth to the analysis, making this a more robust phylogeny and helping to resolve some of the more poorly supported nodes. Based on recent work by Gibson et al. $(2010 ; 2011)$, we suggest that AATS, parts of CAD and cytochrome b would be good markers to use in future studies. Fresh material from all known species needs to be collected and preserved in alcohol at 80 degrees to assist in this endeavour.

Chapter 4: Species Concepts and Key

A neighbour-joining tree of $354 \mathrm{COI}$ sequences was produced (Fig. 4). Intraspecific variation and nearest neighbour values were calculated using BOLD Data Systems Nearest Neighbour.Summary analysis. The results are reported in Table 2.

4.1 Generic Redescription

Dasysyrphus Enderlein, 1938 
Figures: 1 (field photos), 5 - 11 (habitus photos), 12-17 (genitalia), $18-26$ (range maps)

Synonyms and References:

Conosyrphus Matsumura, 1918: 11 (name preoccupied by Conosyrphus Frey, 1915).

Type species Conosyrphus okunii Matsumura

Dasysyrphus Enderlein, 1932: 208. Type species Scaeva albostriata Fallén, 1817: 42

Syrphella Goffe, 1944: 129. Type species Scaeva tricincta Fallén, 1817

Dendrosyrphus Dusek \& Laska, 1967: 365. Type species Syrphus lunulatus Meigen, 1822

Diagnosis: Eye pilose; metasternum bare; wing densely microtrichose, at least at apical $1 / 3$; abdomen oval with abdominal margin present, dark with light maculae present on tergites $2,3 \& 4$.

Description:

Body Length: $5.0-11.7 \mathrm{~mm}$; Wing Length: $4.5-11.4 \mathrm{~mm}$

Head: Frons dark with light pollenose fascia (in males it runs along ventral edge of where eyes meet, in females it is variable and can be diagnostic); face light, typically with dark vitta $1 / 4-1 / 2$ the width of the face, reaching antennal socket or not; face with medial tubercle, more prominent in some species than others; gena dark or 
light; occiput dark, covered in light pollen, pile typically light; eyes covered in dense pile, male holoptic, female dichoptic; scape, pedicel and flagellomere light to dark, arista bare.

Thorax: Scutum dark, sometime shiny or metallic; scutellum variable in colour; wings densely microtrichose on apical $1 / 3$, location and extent of bare areas on basal $3 / 3$ varies between species; pterostigma elongate and dark (brown); haltere light, sometimes bulb darkened; calypter light with long light pile around edge and short light pile on both dorsal and ventral surfaces; legs cylindrical, femora typically basally dark, apically light, tibiae light, metatibia sometimes with dark band close to apex, tarsi light usually with darker anterior side.

Abdomen: Oval; tergites dark with light maculae, maculae on tergite 2 typically oval, while maculae on tergites $3 \& 4$ are typically longer, arcuate, lunulate transverse or oblique, sometimes meeting medially, maculae of some species cross abdominal margin, in others they do not (Figs 5-11); sternites light with dark fasciae, sometimes fascia absent on sternite 2 ; terminalia dark.

Male Genitalia: Hypandrium roughly shaped like a trapezoid, smooth, without pile; epandrium similarly shapes (Figs 12A-17B), smooth, without pile; surstylus more or less triangular in shape in lateral view, flattened posteriorly or anteroposteriorly, long pile on dorsal, laterodorsal and posterodorsal sides, row of spines on posteriomedial edge; cercus oval with long pile over entire surface; gonostylus 
variably shaped; basiphallus variable in shape, but roughly shaped like a faucet, posterodorsal edge can be bare or be covered in pile or spines; distiphallus elongate, basal end flared out into horn in most species, in D. albostriatus and $D$. eggeri it tapers to a point, it is mostly sclerotized but membranous at apex of the horn, with either setae, pile or bare dorsally (12A-17B)

Etymology: From the Greek, dasys, meaning thick with hair, hairy, shaggy; and syrphos (masculine), meaning a kind of fly.

Distribution: Throughout Nearctic and Palaearctic (excluding North Africa). Throughout the Neotropical and Oriental regions, however there is low diversity in these regions. Not recorded from the Afrotropical or Australasian regions. (Figs 1826)

\subsection{Key to Species}

\section{Key to Species of Nearctic Dasysyrphus Enderlein, 1938}

1. Tergites $3 \& 4$ with maculae that extend over the abdominal margin, usually reaching the edge of the abdomen

$1^{\prime}$. Tergites $3 \& 4$ with maculae that do not reach the abdominal margin . .2 . 
2'. Gena light in colour (yellow to light brown)

3. Tergites $3 \& 4$ with maculae arcuate, medial edge flattened, usually broadly contacting the anterior edge of the tergite (Figs 5C, D \& 13A) creper (Snow) Western (southern AB \& BC, south to CA \& NM; Fig. 18); Snow, 1895: 234. 3'. Tergites $3 \& 4$ with maculae oblique, more straight, with medial edge rounded and usually meeting in medially, sometimes contacting anterior edge of tergite, but not as broadly.

4. Gena separated from face by black vitta; wing cells br and bm almost entirely bare (Figs. 9B \& D) richardi (Locke \& Skevington) sp. nov. Southwestern (NM; Fig. 20); Locke \& Skevington, here described. 4'. Gena and face continuously yellow, not separated by black vitta; wing cells br and bm partly microtrichose (Figs 9A, C \& 14A) lotus (Williston) Southwestern (CA \& AZ, south to Mexico; Fig. 22); Williston, 1887: 75.

5. Sternite 2 with dark oval macula (Figs 11C, D \& 16B) ..pinastri (DeGeer) Greenland (Fig. 25); De Geer, 1776: 113, Pl. 7, Figs 1-7. $5^{\prime}$. Sternite 2 with dark fascia 6.

6. Tergites $3 \& 4$ with maculae not greatly constricted, more or less uniform in thickness. 8. 
6'. Tergites 3 \& 4 with maculae greatly constricted medially, sometimes completely divided. 7.

7. Male surstylus with a prominent posterolateral edge. Cell br usually with bare areas above base of spurious vein (sometimes very small); tergites $3 \& 4$ with maculae usually divided (Figs 10A, B, C \& 12B)

occidualis (Locke \& Skevington) sp. nov.

Western (AK, south to CO; Fig. 23); Locke \& Skevington, here described.

$7^{\prime}$. Male surstylus without a prominent posterolateral edge . Cell br densely microtrichose, tergites $3 \& 4$ with maculae either divided or not (Figs 5 A, B, \& 12A) amalopis (Osten Sacken) Northern (YK, east to northern NL; NH; Fig. 18); Osten Sacken, 1875: 148.

8. Tergite 2 with anterolateral corner of maculae extending anterolaterally into a point, in females reaching the edge of the abdomen; profemur light, usually entirely so but some dark basally (Figs 7D, E, F \& 15A) laticaudus (Curran) Eastern and boreal (AK, east to NL, south to NH, Fig. 20); Curran, 1925: 175, PI. XI, Fig. 151.

8'. Tergite 2 with anterolateral corner of maculae not extending anterolaterally into a point, lateral edges of maculae usually rounded; profemur dark basally, light apically to entirely dark 9. 
9. Small, dark flies; profemur $1 / 2$ - entirely dark; female frons complete, but sometimes not well defined; males with tergite 2 maculae typically reduced and positioned laterally; tergites $3 \& 4$ with maculae thick and slightly narrowed medially (Figs 7A, B, C \& 14B). nigricornis (Verrall) Northern (AK, east to northern NL; Greenland; Fig. 22); Verrall 1873: 251. 9'. Not as above. 10.

10. Male gonostylus with narrow, wavy projection on posterodorsal surface. Wing typically largely bare basally, with cells br and bm with large dense areas (Figs 10D, $E \& 15 B)$ pacificus (Lovett) Western, (southern BC, south to CA; Sierra Nevada, Cascades and Costal Mountain Ranges; Fig. 24); Lovett (in Cole \& Lovett), 1919: 245.

$10^{\prime}$. Male gonostylus with projection on posterodorsal surface straight; wing typically densely microtrichose, sometimes with bare areas in cells $r_{1}, b r, b m$ and/or c (Figs 11A, B \& 16A) pauxillus (Williston) Western (AK, south to NM; Rocky Mountain Range; Fig. 25); Williston, 1887: 74.

11. Tergites $3 \& 4$ with maculae transverse, usually narrow, with slight swelling on medial edge; tergite 2 with maculae very small, $<1 / 4$ width of tergite 2 (Figs 8 C, D \& 13B) limatus (Hine) Widespread (AK, east to NS, south to CO and MA; Fig. 21); Hine, 1922: 146. 
11 '. Tergites $3 \& 4$ with maculae distinctly arcuate or lunulate, with at least slight swelling on medial edge, if tergites $3 \& 4$ with maculae more straight then tergite 2 with maculae large, $>1 / 3$ width of tergite 2

12. Tergites $3 \& 4$ with maculae distinctly arcuate or lunulate, with anterior edge forming a distinct $\mathrm{U}$ or $\mathrm{V}$ shape, maculae are distinctly constricted, sometimes dividing in half with a large swelling on the medial edge (Figs $6 \mathrm{~A}-\mathrm{F} \& 17 \mathrm{~A}$ ) ...intrudens complex (Osten Sacken) Widespread (AK, east to NL, south to CA, NM and TN; Fig. 19); Osten Sacken, 1877: 326.

12'. Tergites $3 \& 4$ with maculae are more oblique, no distinctive constriction in the centre, never divided and not greatly swollen on medial edge (Figs 8A, B \& 17B)...... venustus (Meigen)

Widespread (AK, east to NS, south to NM \& NC; Fig. 26); Meigen, 1822: 299.

4.3 Species Descriptions

\section{Dasysyrphus amalopis (Osten Sacken)}

Figures: 5A, B, 12A \& 18

Synonyms and References:

Syrphus amalopis Osten Sacken, 1875: 148 (original description) 
Curran, 1925: 179 (discussion, amalopis s.l.)

Metasyrphus amalopis (Osten Sacken):

Fluke, 1933: 110 (in part; key, redescription and transfer to Metasyrphus) Dasysyrphus amalopis (Osten Sacken):

Stone et al., 1965 (in part): 563 (catalogue and transfer to Dasysyrphus)

Vockeroth, 1986: 202 (lectotype designation and discussion)

Vockeroth, 1992: 63 (in part; redescription)

Diagnosis: Abdominal maculae do not meet abdominal margin; maculae on tergites $3 \& 4$ are constricted medially and usually not divided (Figs 5A \& B). Wings cells are densely microtrichose. Surstylus without a prominent posterolateral edge (Fig. 12A). Similar to $D$. occidualis, but $D$. occidualis has maculae on tergites $3 \& 4$ either divided or undivided (Figs $10 \mathrm{~A} \& \mathrm{~B}$ ), cell br usually with a small bare area above base of spurious vein and a prominent posterolateral edge on the surstylus. (Fig. 12B).

Description:

Body Length: $7.8-10 \mathrm{~mm}$; Wing Length: $7.7-8.7 \mathrm{~mm}$

Head: Frons dark with light pollenose fascia (in males it runs along ventral edge of where eyes meet, in females it is continuous, $1 / 1,-1 / 2$ the length between the antennae and ocelli) and dark pile; face light with dark vitta $1 / 4-1 / 3$ the width of the face 
sometimes reaching antennal socket, face with dark pile; gena dark with pile light to dark; occiput dark, covered in light pollen, pile light; scape, pedicel and flagellomere dark.

Thorax: Scutum dark, sometime shiny or metallic, pile dark and light, more light anteriorly and posteriorly; scutellum dark with darker lateral edges, light pile along anterior edge and dark pile elsewhere; wing densely microtrichose, no bare areas in cells; haltere light; femora basally dark, apically light, tibiae light basally and dark apically, tarsi dark.

Abdomen: Maculae on tergite 2 large and oval, usually with slight narrowing medially, $1 / 3-<1 / 2$ the width of tergite 2 , maculae on tergites $3 \& 4$ are oblique, constricted medially and usually not divided, posterior edge of maculae straight, maculae do not reach abdominal margin (Fig. 5A); sternite 2 is light with a dark triangular macula, usually not connecting with the lateral edges of the sternite, sternites $3 \& 4$ are light with dark fasciae.

Male Genitalia: Surstylus more or less triangular in shape in lateral view, flattened posteriorly, long pile on dorsal, laterodorsal and posterodorsal sides, row of spines on posteriomedial edge; cercus oval with long pile over entire surface; gonostylus claw-shaped, pile on dorsal surface; basiphallus elongate, basally bent at about a 90 degree angle towards the anterodorsum, apical end curved ventrally with a widened apex and is partly membranous dorsally, with spines on posterodorsal 
side, apex; distiphallus elongate, apical end flared out into horn, which is mostly sclerotized but membranous at apex with minute setulae dorsally, basally bent towards dorsum and slightly enlarged ventrally at bend (Fig. 12A).

Intraspecific variation: From the specimens I have had the chance to observe the maculae on tergites $3 \& 4$ are usually not divided (Fig. 5A). Sometimes just the maculae on tergite 3 are divided and sometimes they are divided on both tergites 3 \& 4. The lectotype male has entire maculae, whereas the paralectotype female has divided maculae.

Etymology: From the Greek, amalos meaning soft, tender, weak and -opis, meaning having the appearance of, like.

Distribution: Northern Canada (MB, NL, QC, YK) and Northeastern USA (NH) (Fig. 18)

Ecology: Collected from June - July at elevations from sea level-1006m.

Discussion: Along with the two specimens nominated lectotype and paralectotype by Vockeroth (1986), Osten Sacken (1875) discusses a third specimen and includes it in his description of the species. This third specimen has abdominal maculae that cross the abdominal margin. This third specimen is not a specimen of $D$, amalopis but a specimen of the $D$. intrudens complex, and has caused much confusion over the 
name D. amalopis. Vockeroth (1986) pointed out that Fluke (1933) discusses many specimens of $D$. amalopis with maculae reaching the margin. Vockeroth stated that he believed those specimens belonged to his concept of $D$. venustus. All specimens described as $D$. amalopis that have maculae that cross the abdominal margin belong to the $D$. intrudens complex. Fluke (1933) does have D. amalopis key out in two places in his key. The D. amalopis keyed out in couplet 54 pertains to D. amalopis s.s. and $D$. occidualis, while the $D$. amalopis keyed out in couplet 56 pertains to the $D$. intrudens complex.

The lectotype and paralectotype are the only known specimens of this species from eastern North America, south of northern Quebec and Labrador. Dasysyrphus occidualis has often been confused with D. amalopis. However, the COI sequences and male genitalia characters provide evidence for these being two distinct species with an identical habitus. Dasysyrphus amalopis ranges from southern Yukon to northern Labrador (Fig. 18), while D. occidualis ranges from Alaska, south to Colorado (Fig. 23). Specimens referred to as D. amalopis from western Canada or the US are most likely that of $D$. occidualis and in the overlap zone in southern Yukon male genitalia and/or COI sequences should be used to confidently identify specimens. Despite the long standing use of the name $D$. amalopis, the similar new species, $D$. occidualis, has been much more frequently collected.

It is not known why no other specimens are known from as far south as the 
lectotype and paralectotype. They were both collected in the White Mountains, in New Hampshire. The date of their collection is unknown, however their description by Osten Sacken was published in 1875 , so they would have been collected previous to that. It is possible that they are rare and have not been collected again or that their habitat or climatic conditions have changed resulting in their extirpation. More collecting should be done in the Appalachians at high elevation to see if this species still exists this far south.

Type Material: "White Mts./ Austin", "Osten/ Sacken/ Coll.", red label "Type/ 4092", "Syrphus/ amalopis/ Type Species O.S.", yellow label "LECTOTYPE/ Syrphus/ amalopsis O.S./ Desig. Thompson 1971", "Jan.-July 2003/ MCZ Image/ Database", J. Skevington/ Specimen \#/ 23785", male, deposited in the Museum of Comparative Zoology, Harvard University (MCZ). Missing head. (examined)

Material Examined:

Paralectotype: United States of America: New Hampshire: Gorham, [44.387788 N, -71.17313 W], 1 female, JSS 23786 (MCZ). Other Material Examined: Canada: Manitoba: Churchill Region; Weir, [58.4026 N, -94.129 W], 1m, 25.vii.2007, 1 female, 07PROBE 4723, L.E. Roscoe (CNC); Churchill Area, Burn site N[orth] of Twin lakes, [58.61861111 N, -93.82888889 W], 5.vii.2007, 1 male, CNCD 9607, J. Skevington (CNC); 26km SE Churchill, Twin Lakes, [58.63 N, -93.819 W], 14m, 19.vii.2009, 1 female, 09PROBE 176, Arctic Ecology 2009 (CNC); 26km SE Churchill, Twin Lakes, [58.63 N, -93.819 W], 14m, 26.vi.2010-2.vii.2010, 1 male, 10PROBE 
13673, J. Wang (CNC); 26km SE Churchill, Twin Lakes, [58.63 N, -93.819 W], 14m, 5.vii.2010-9.vii.2010, 1 female, 10PROBE 13805, J. Wang (CNC); $11 \mathrm{~km} \mathrm{~S} \mathrm{Churchill;}$ Goose Creek, [58.649 N, -94.193W], 19.vii.2009, 1 female, 09PROBE 25, A. Thielman (CNC); 10km SE Churchill, Farnworth Lake (Landing L), [58.7045 N, 94.0525 W], 14m, 14.vii.2010, 1 female, 10PROBE 14269, J. Wang (CNC); 10km SE Churchill, Farnworth Lake (Landing L), [58.7045 N, -94.0525 W], 14m, 14.vii.201019.vii.2010, 1 male, 10PROBE 15042, J. Wang (CNC); 10km SE Churchill, Farnworth Lake (Landing L), [58.7045 N, -94.0525 W], 14m, 28.vi.2010, 1 male, 10PROBE 10909, J. Wang (CNC), 28.vi.2010-6.vii.2010, 1 female, 10PROBE 15283, J. Wang (CNC); Newfoundland and Labrador: Nain, Labrador, Anatalak Bay, [56.5429 N, 61.689989 W], 16.vii.1928, 1 male, JSS 19248, A.C. Weed (FMNH ); Quebec: Indian House Lake, [56.328482 N, -64.720845 W], 19.vii.1954; 1 female, CNCD 13616, W.R. Richards (CNC); Indian House Lake, [56.328482 N, -64.720845 W], 22.vii.1954, 1 female, CNCD 13617, R. Coyles (CNC); Indian House Lake, [56.328482 N, 64.720845 W], 25.vii.1954, 1 female, CNCD 13618, W.R. Richards (CNC); Yukon Territory: La Force Lake, [62.683333 N, -132.333333 W], 1006m, 26.vi.1960, 1 male, CNCD 13587, J.E.H. Martin (CNC).

\section{Dasysyrphus creper (Snow)}

Figures: 5C, D, 13A \& 18

Synonyms and References: 
? Syrphus lotus Williston, 1887: 75 var. (short description of a variety of lotus)

Syrphus pauxillus Snow, 1892: 37 (nec. Williston; short description)

Syrphus creper Snow, 1895: 234 (original description)

Syrphus lotus Curran, 1925: 92 Pl. VI Fig. 51 (nec. Williston; redescription)

Syrphus lotus var. creper Curran, 1925: 180 (discussion)

Metasyrphus creper (Snow):

Fluke, 1933: 107 (key, redescription and transfer to Metasyrphus)

Dasysyrphus creper (Snow):

Stone et al., 1965: 563 (catalogue and transfer to Dasysyrphus)

Vockeroth, 1986: 202 (lectotype designation)

Vockeroth, 1992: 65 (key and redescription)

Diagnosis: Abdominal maculae do not meet abdominal margin; maculae on tergites $3 \& 4$ arcuate with medial edge flattened and usually broadly contacting anterior edge; markings unlike any other species (Fig. 5C, D).

Description:

Body Length: 6.7 - 10.5mm; Wing Length: $6.3-9.2 \mathrm{~mm}$

Head: Frons dark with light pollenose fascia (in males it runs along ventral edge of where eyes meet, in females it is $1 / 2$ the length between the antennae and ocelli) and dark pile; face light with dark vitta $1 / 4-1 / 3$ the width of the face, usually not reaching 
antennal socket, face with light pile, dark pile lateral to the eye; gena light (Fig. 5D) with light pile and usually some dark pile below eye; occiput dark, covered in light pollen, pile light; scape and pedicel light to dark, flagellomere dark.

Thorax: Scutum dark, sometime shiny or metallic, pile light; scutellum light to dark with darker lateral edges, light pile along anterior edge and dark pile elsewhere; wing cells br and bm entirely bare, cell $c$ bare from base to $3 / 4, r_{1}$ bare at base and cup bare at base and along vein CuP; haltere light; femora basally dark, apically light, tibiae light, metatibia sometimes with dark band close to apex, tarsi light with darker anterior side.

Abdomen: Maculae on tergite 2 large and oval, maculae on tergites $3 \& 4$ are arcuate, with medial edge widened, flattened and usually broadly contacting the anterior edge of tergite, sometimes meeting medially, maculae do not reach abdominal margin (Figs 5 C, D); sternites light with dark fasciae.

Male Genitalia: Surstylus more or less triangular in shape in lateral view, flattened posteriorly, long pile on dorsal, laterodorsal and posterodorsal sides, row of spines on posteriomedial edge; cercus oval with long pile over entire surface; gonostylus boot-shaped with projection on posterodorsal surface that projects dorsally, pile on dorsal surface; basiphallus elongate, basally bent at 90 degree angle towards dorsum, apical end curved ventrally and is partly membranous with pile on dorsa! side; distiphallus elongate, apical end flared out into horn, which is mostly 
sclerotized but membranous at apex with minute setulae dorsally, basally bent towards dorsum and slightly enlarged at bend (Fig. 13A).

Intraspecific variation: Scutellum colour varies from light to dark; maculae on tergites $3 \& 4$ sometimes meet medially and sometimes are separated, medial edge usually broadly contacts anterior edge of tergite but if not then it comes close to anterior edge and edge of maculae are rounded; dark fasciae on sternites vary in width.

Etymology: The word creper is Latin, meaning dark or obscure.

Distribution: Southwestern Canada $(A B, B C)$ and western USA (AZ, CA, CO, ID, MT, NM, OR, SD, UT, WA, WY). (Fig. 18)

Ecology: Collected on ponderosa pine (Pinus ponderosa Douglas ex Lawson \& C. Lawson, Pinaceae), sierra willow (Salix orestera C.K. Schneid., Salicaceae), willow (Salix Linnaeus, Salicaceae), chokecherry (Prunus virginiana, Rosaceae), Ross' avens (Geum rossii (R. Brown) Seringe, Rosaceae), avens (Geum Linnaeus, Rosaceae), tall ragwort (Senecio serra Hooker, Asteraceae), threenerve goldenrod (Solidago velutina DC., Asteraceae), rabbitbush (Chrysothamnus Nuttall, Asteraceae). Habitats listed on labels include "along timberline", "creek margin" and "on tundra". The specimen collected on the "tundra" was collected in Colorado at $3505 \mathrm{~m}$, so this is in reference to an alpine tundra habitat. Collected April to October, although much 
more frequently collected in July and August. Collected from elevations of $305 \mathrm{~m}-$ $3688 \mathrm{~m}$, although most frequently collected from elevations above $2000 \mathrm{~m}$.

Discussion: Presumably Williston (1887) was referring to a specimen of $D$. creper when he described the female from New Mexico as a second specimen in his description of lotus. He noted there were differences but that it was "perhaps allied" with the female from Arizona that he described as lotus. He describes the abdominal maculae of this second specimen as "more oblique, and strongly concave in front" and "more convex behind" and the gena as having a "black stripe", which are characters more befitting of Snow's description of creper, which came some seven years later.

Three years before Snow formally described creper in 1895 he described specimens as pauxillus (Snow 1892). In his description of creper he synonymizes his 1892 pauxillus description with creper. Snow is also the one who suggests Williston's (1887) variety of lotus is probably creper.

In Curran's monograph (Curran 1925) he demotes S. creper from the rank of species to a variety of $S$. lotus. He claimed that there were no differences between the two except for the amount of interruption in the second and third abdominal bands. He believed it was just an extreme in coloration and did not warrant the rank of species. However, in the same paper he provides a redescription of lotus and based on the characters discussed and illustrated (Plate VI: 51), it is clear that his 
concept of lotus is not the same as Williston's and he is actually describing creper. In 1933, Fluke re-elevated creper to the rank of species. Fluke states that at that time lotus was only known from the type and that published records of lotus probably refer to creper. Fluke also notes that Curran was probably looking at specimens of creper, not lotus.

Fluke (1933) clearly distinguishes between these two species based on the abdominal markings, with lotus having more straight bands and those of creper being more arcuate. Fluke also places both species into Metasyrphus and into the amalopis group with other species currently recognized as Dasysyrphus.

Type Material: "Magdalena,/ New Mexico", "Type", blank red label, red label "Syrphus/ creper Snow/ LECTOTYPE/ Des. Vockeroth/ 1984", "Jeff Skevington/ Specimen \#/ 23782"; male; deposited in the Snow.Entomological Museum, University of Kansas. (examined)

Material Examined:

Paralectotypes: United States of America: Colorado: Estes Park, [40.376877 N, 105.52137 W], viii.1892, 1 male, JSS 20203, F.H. Snow (FMNH); Estes Park, [40.377201 N, -105.521661 W], viii.1892, 1 male, OSUC 319691, F.H. Snow (OSU); Other Material Examined: Canada: Alberta: 5 km South of Elkwater, Hwy 41, [49.65 N, -110.2666667 W], 7.vii.1982, 1 female, JSS 18293, B.V. Peterson (CNC); Medicine Hat, Division No. 1, [50.040483 N, -110.673523 W], 15.v.1930, 1 male,JSS 
19477, F.S. Carr (CNC); Kananaskis, Forest Experimental Station Seebe, [51.100879 N, -115.087692 W], 15.vi.1968, 1 male, CNCD 13634, H.J. Teskey (CNC); Kananaskis, Forest Experimental Station Seebe, [51.100879 N, -115.087692 W], 20.vi.1950, 1 female, CNCD 13643 (CNC); Sulphur Mountain, Banff National Park, [51.149438 N, 115.583484 W], 2195m, 28.vii.1967, 21 males, CNCD 13635, 13636, J.R. Vockeroth (CNC); Banff, Banff National Park, [51.180275 N, -115.568433 W], 27.vi.1925, 1 female, JSS 19442, 0. Bryant (CNC); Snow Creek Pass, Banff National Park, [51.60524 N, -115.808617 W], 2256m, 28.vii.1962, 1 female, CNCD 13642, K.C. Herrmann (CNC); Snow Creek Pass, Banff National Park, [51.60524 N, -115.808617 W], 2256m, 13.viii.1962, 1 female, CNCD 13644, K.C. Herrmann (CNC); Peyto Lookout, Banff National Park, [51.717631 N, -116.508919 W], 2438m, 23.vii.1962, 1 male, CNCD 13630, 1 female, CNCD 13641, K.C. Herrmann (CNC); British Columbia: Glacier Lake, Cathedral Provincial Park, [49.055785 N, -120.207299 W], 6.vii.1986, 1 male, CNCD 13631, R.A. Cannings (CNC); Cathedral Prov. Park, Glacier Lake, [49.055785 N, -120.207299 W], 7.vii.1986, 1. male, Royal British Columbia Museum ENT 9881939, 1 female, Royal British Columbia Museum ENT 9881942, R.A. Cannings (RBCM); Manning Provincial Park, Valleyview, OkanaganSimilkameen R.D., [49.07995 N, -120.766842 W], 1830m, 14.vii.1986, 1 male, JSS 18368, S.G. Cannings (CNC); Cathedral Prov. Park, Quiniscoe Mtn. summit, [49.08 N, -120.18 W], 26.vii.1988, 1 male, JSS 20170, S.G. Cannings (UBCZ); Manning Park, Blackwall Mountain, Okanagan-Similkameen,R.D., [49.098666 N, -120.766925 W], 1828m, 7.viii.1953, 1 male, CNCD 13632, D.F. Hardwick (CNC); Mount Kobau, Osoyoos, Okanagan-Similkameen R.D., [49.111594 N, -119.664702 W], 9.vii.1968, 1 
male, CNCD 13627, 1 female, CNCD 13640, D. Allen (CNC); Mount Kobau, Osoyoos, Okanagan-Similkameen R.D., [49.111594 N, -119.664702 W], 1890m, 13.viii.1967, 1 male, CNCD 13628, J.R. Vockeroth (CNC); Oliver, Okanagan-Similkameen R.D., [49.182338 N, -119.550442 W], 305m, 23.viii.1953, 1 female, CNCD 13637, J.E.H. Martin (CNC); Oliver, Okanagan-Similkameen R.D.; [49.182338 N, -119.550442 W], 14.ix.1923, 1 female, CNCD 13638, C.B. Garrett (CNC); Robson, Cascade, [49.342029 N, -117.697831 W], 22.vi.1959, 1 female, CNCD 13639, H.R. Foxlee (CNC); Hedley, Okanagan-Similkameen R.D., [49.357784 N, -120.075962 W], 7.viii.1953, 1 male, CNCD 13633, D.F. Hardwick (CNC); Penticton Apex Mountain, Jct of powerline road to summit, [49.482486 N, $-119.934458 \mathrm{~W}], 1900 \mathrm{~m}, 15 . v i i .1988,1$ male, Royal British Columbia Museum ENT 9885672, R.A. Cannings (RBCM); Cranbrook, Elizabeth Lake, [49.497195 N, -115.791152 W], 6.vii.1980, CNCD 60067, R.A. Cannings (CNC); First Summit, Mount Harry, Mount Revelstoke National Park, [51.035979 N, -118.142509 W], 2316m, 20.viii.1952, 1 male, CNCD 13629, G.P. Holland (CNC); United States of America: Arizona: Rustler Park, w. Portal, [31.90556 N, -109.27917 W], 2560m, 2.viii.1977, 1 female, USNM ENT 258093, CW Sabrosky (USNM); Rustler Park, Cochise County, [31.906851 N, -109.278543 W], 2560m, 13.ix.1965, 1 female, JSS 19475, C.W. Sabrosky (CNC); South Arizona, [32.948115 N, -111.88073 W], viii.1902, 21 females, CNCD 26379, JSS 19474, C.W. Johnson (CNC); S. Arizona, [32.948115 N, -111.544233 W], viii.1902, 1 male, JSS 21342, 1 female, JSS 21340, F.H. Snow (MCZ); Bradshaw Mountains, [34.41472222 N, -112.4038889 W], 1951m, 7.v.1999, 1 male, ISS 20683, R.S. Beal (CSUC); Oak Creek Canyon, Coconino County, [34.961452 N, -111.753046 W], 1 female, CNCD 
26378, F.H. Snow (CNC); Weimer Spring, Coconino County, [34.973632 N, 111.528763 W], 4.vi.1963, 1 male, JSS 19445, C.H. Spitzer (CNC); San Francisco Mountains, Flagstaff, [35.340306 N, -111.670881 W], 3505m, 14.viii.1934, 1 male, JSS 19435, 1 female, JSS 19436, E.L. Bell (CNC); North Rim of Grand Canyon, [36.17849 N, -113.226775 W], 17.viii.1966, JSS 25081, W.J. Hanson (EMUS);

California: Quaking Aspen, Riverside Co., [33.69554 N, -117.163778 W], 5.vii.1984, 1 male, JSS 18296, D.J. Burdick (CNC); San Jacinto Mountains, Riverside County, [33.814491 N, -116.679201 W], 1 female, JSS 19459, J.M. Aldrich (CNC); Westwood, Los Angeles County, [34.056121 N, -118.430635 W], 17.vi.1959, 1 female, CNCD 26374, Kelton \& Madge (CNC); Upper Santa Ana River, San Bernardino County, [34.1003 N, -117.020931 W], 7.vii.1957, 31 males, JSS 19466-19468, 21 females, JSS 19438, 19465, A.L. Melander (CNC); Glacier Point Road, Yosemite National Park, [37.673917 N, -119.637698 W], 1.vii.1947, 1 female, JSS 19464, A.L. Melander (CNC); Yosemite, [37.741951 N, -119.577767 W], 1183m, 26.v.1931, 1 male, JSS 20584, (EMEC); Mather Site, Buck Meadows, Tuolumne County, [37.880173 N, 119.856977 W], 1970, 1 male, CNCD 26352, 1 female, CNCD 26375, A.R. Moldenke (CNC); Tioga Pass, Hall Area site, Mono County, [37.919391 N, -119.255072 W], 26.vii.1969, 21 females, CNCD 26376, 26377, A.R. Moldenke (CNC); Calaveras Big Trees SP, S. grove, banks of Beaver Creek, MT \#2, [38.26166667 N, -120.2577778 W], 1375m, 22.v.-11.vi.2007, 31 males, JSS 20954-20956, 1 female, JSS 20951, A.R. Cline \& P.H. Kerr (MHPC); Wright's Lake, [38.84944444 N, -120.2269444 W], 2120m, 20.vi.2006, 1 male, JSS 20962, M. Hauser, S. Winterton, \& E. Fisher (MHPC); Yuba Pass, [39.323059 N, -120.599169 W], 2042m, 9.vii.1954, 1 female, JSS 20589, 
R.H. Goodwin (EMEC); So. Calif., v.1908, 41 males, JSS 20585-20588, R. Woglum (EMEC); Blanco's Corral White Mt., 3048m, 30.vi.1953, 1 female, JSS 20590, J.W. MacSwain (EMEC); Truckee, 25.vi.1943, 31 males, JSS 23769, 23771, 23772, 1 female, JSS 23770, P.H. Arnaud (USNM); Colorado: 10 Miles East of Silverton, San Juan County, [37.809565 N, -107.485032 W], 2743m, 13.viii, 1 male, CNCD 26351, F.M. Carpenter (CNC); Bennet Crk., [37.941103 N, -108.309527 W], 7.ix.1933, 1 female, JSS 20707 (CSUC); Garfield, [38.55185 N, -106.292119 W], 3658m, 5.viii.1971, 1 female, USNM ENT 258077, W.E. Steiner (USNM); Paonia, [38.86832 N, -107.592002 W], 16.ix.1912, JSS 20711, Chas L. Fluke (CSUC); Colorado State, [38.98829 N, -105.827992 W], 41 males, JSS 19450-19453, 21 females, JSS 19454, 19455 (CNC); Independence Pass, Lake County, [39.104722 N, -106.557321 W], 3688m, 31.vii.1961, 1 male, CNCD 26354, B.H. Poole (CNC); Mount Evans, Park County, [39.25 N, -106.1666667 W], 3658m, 3.viii.1961, 1 male, CNCD 26355, 1 female, CNCD 26368, S.M. Clark (CNC); Tennessee Pass, Lake County, [39.357246 N, -106.313889 W], 3131m, 7.vii, 1 female, JSS 19458, J.M. Aldrich (CNC); Tenmile Range, unamed Cr. avalanche chute W side, [39.425 N, -106.111 W], 3200m, 15.vii.17.vii.1998, 1 male, JSS 20682, S. Fitzgerald \& A. Foley (CSUC); Rogers Pk., [39.615821 N, -105.61778 W], 3660m, 12.vii.1997, 1 male, JSS 20805, C. Slater (CSUC); Rogers Pk., [39.615821 N, -105.61778 W],.3660m, 10.viii.2002, 1 female, JSS 20806, C. Slater (CSUC); Timberline, Mount Evans, Clear Creek County, [39.645575 N, -105.594897 W], 3566m, 22.vii.1961, 1 female, CNCD 26367, B.H. Poole (CNC); Arapaho National Forest, Mt. Evans, Echo Lake, Path on west side of lake, [39.659767 N, -105.604767 W], 3176m, 29.vi.2010, 21 males, CNCD 60033- 
60034, 1 female, JSS 24626, M.M. Locke (CNC); Doolittle Ranch, Mt. Evans, Clear Creek County, [39.675739 N, -105.601348 W], 2987m, 10.vii.1961, 1 female, CNCD 26364, W.R.M. Mason (CNC); Doolittle Ranch, Mt. Evans, Clear Creek County, [39.675739 N, -105.601348 W], 2987m, 3.viii.1961, 1 female, CNCD 26365, W.R.M. Mason (CNC); Nederland, Science Lodge, Boulder County, [40.031645 N, 105.533886 W], 2896m, 1.vii.1961, 1 male, CNCD 26357, A.A. Bucknell (CNC); Nederland, Science Lodge, Boulder County, [40.031645 N, -105.533886 W], 2896m, 28.vi.1961, 1 female, CNCD 26361, W.R.M. Mason (CNC); Nederland, Science Lodge, Boulder County, [40.031645 N, -105.533886 W], 2896m, 6.vii.1961, 1 female, CNCD 26362, B.H. Poole (CNC); Nederland, Science Lodge, Boulder County, [40.031645 N, -105.533886 W], 2896m, 1.vii.1961, 1 female, CNCD 26363, B.H. Poole (CNC); Niwot Ridge, Near Ward, Boulder County, [40.069933 N, =105.607397 W], 3505m, 4.vii.1961, 1 male, CNCD 26356, 1 female, CNCD 26366, C.H. Mann (CNC); Niwot Ridge, Near Ward, Boulder County, [40.069933 N, -105.607397 W], 7.vii.1965, 1 female, JSS 19446, B. Petersen (CNC); Ward, [40.072208 N, -105.508332 W], 9.viii.1913, 1 male, JSS 20712, (CSUC); Estes Park, [40.376877 N, -105.52137 W], 8.viii.1931, 1 female, JSS 20669, (CSUC); Estes Park, [40.376877 N, -105.52137 W], 20.v.1933, 1 female, JSS 20671, (CSUC); Estes Park, [40.376877 N, -105.52137 W], viii.1892, 1 male, JSS 21335, F.H. Snow (MCZ); Rocky Mountain National Park, Fall R. entrance, [40.40344 N, -105.589203 W], 4.vi.1999, 1 female, JSS 20684, D. Leatherman (CSUC); Rocky Mountain National Park, Fall R. entrance, [40.40344 N, 105.589203 W], 1.viii.1997, 1 female, JSS 20685, D. Leatherman, Manhattan, W. Rustic (CSUC); Masonville, [40.487171 N, -105.210056 W], 5.ix.1933, 1 female, JSS 
20674, (CSUC); Cameron Pass, [40.520816 N, -118.227668 W], 7.vii.1931, 1 female, JSS 20709, (CSUC); Cameron Pass, [40.520816 N, -118.227668 W], 3048m, vii, 1 male, JSS 19981, (FMNH); Pingree Park, [40.561094 N, -105.597778 W], 19.viii.1932, 1 male, JSS 20668, C.M. Drage (CSUC); Pingree Park, [40.561094 N, 105.597778 W], 20.viii.1924, 1 female, JSS 20710, Chas L. Fluke (CSUC); Lory State Park Well Gulch Trail, [40.572996 N, -105.191669 W], 18.vi.1995, 1 female, JSS 20809, D. Leatherman (CSUC); Fort Collins, [40.58333333 N, -105.0833333 W], 5.x.1921, 1 female, JSS 20676, (CSUC); Ft. Collins, [40.58333333 N, -105.0833333 W], 19.ix.1932, 1 female, JSS 20680, (CSUC); Ft. Collins, [40.58333333 N, 105.0833333 W], 21.ix.1932, 1 female, JSS 20697, (CSUC); Ft. Collins, [40.58333333 N, -105.0833333 W], 20.ix.1932, 1 female, JSS 20698, (CSUC); Ft. Collins, [40.58333333 N, -105.0833333 W], 30.ix.1942, 1 female, JSS 20703, R.F. duChanois (CSUC); Ft. Collins, [40.58333333 N, -105.0833333 W], 8.ix.1913, 1 female, JSS 20742, (CSUC); Ft. Collins, [40.58333333 N, -105.0833333 W], 19.ix.1942, 1 female, JSS 20743, R.F. duChanois (CSUC); Chambers Lake, [40.603663 N, -105.853645 W], 25.vi.1932, 1 male, JSS 20672, 2 females, JSS 20675, 20701, C.R. Jones (CSUC); North Park, [40.73 N, -106.18 W], 1.vii.1932, 3 males, JSS 20695, 20696, 20705, 6 females, ISS 20670, 20673, 20677, 20678, 20699, 20700, (CSUC); Glendevey, [40.8078 N, 105.935501 W], 7.vii.1932, 1 female, JSS 20679, C.R. Jones (CSUC); Glendevey, [40.8078 N, -105.935501 W], 3.vii.1932, 1 male, JSS 20704, C.R. Jones (CSUC); Glendevey, [40.8078 N, -105.935501 W], .iv.1931, 1 female, JSS 20708, (CSUC); Homer, 7.ix.1934, 1 female, JSS 20706, (CSUC); Boulder Canyon, 2377m, 8.viii.1960, 1 female, JSS 21113, R.\&K. Dreisbach (MSUC); Pingree Park, 14.viii.1934, 1 male, JSS 
23764, C.W.Sabrosky (USNM); Idaho: 4th of July Creek, Custer County, [44.39658 N, -114.672022 W], 27.vii.1975, JSS 25139, T. Griswold (EMUS); Mountains Near Moscow, Latah County, [46.807258 N, -116.868969 W], 9.vii.1911, 1 male, CNCD 26358, 1 female, CNCD 26373, F.M. Hull (CNC); Mountains Near Moscow, Latah County, [46.807258 N, -116.868969 W], 8.vii.1911, 1 female, JSS 19470, (CNC); Mountains Near Moscow, Latah County, [46.807258 N, -116.868969 W], 6.vii.1912, 1 female, JSS 19471, (CNC); Mountains Near Moscow, Latah County, [46.807258 N, 116.868969 W], 5.vii.1912, 1 male, JSS 19472, (CNC); Craig's Mountain, 29.iv.1916, 1 female, JSS 21330, (MCZ); 4 mi. N.W. Holbrook, 16.v.1969, JSS 25080, G.F. Knowlton \& G.E. Bohart (EMUS); Montana: Missoula County, Missoula, [46.871403 N, -113.994267 W], 22.viii.1904, 1 female, JSS 19473, (CNC); New Mexico: Las Cruces, Dona Ana, [32.3 N, -106.7666667 W], 2 males, CNCD 26359, 26360, 2 females, CNCD 26380, 26381, F.M. Hull (CNC); North Fork Ruidoso, White Mountains, Lincoln County, [33.331649 N, -105.672958 W], 2499m, viii.1920, 1 male, JSS 19440, Townsend (CNC); Trout Spring Canyon, San Miguel County, [35.659688 N, -105.34231 W], iv.1927, 1 female, JSS 19449, (CNC); Truchos Peak, [35.974028 N, -105.636663 W], 4.viii, 1 female, JSS 19456, W.P. Cockerell (CNC); Embudo, Rio Arriba, [36.207523 N, -105.961132 W], 25.ix.1897, 1 female, JSS 19441, (CNC); Gallinas River at Las Valles, 3505m, 20.iv, 1 female, JSS 19437, E.L. Bell (CNC); Oregon: Crater Lake National Park, Kalmoth Co., [42.925321 N, 122.172722 W], 10.vii.1968, 1 female, JSS 18297, B.V. Peterson (CNC); 15 mi S Sisters, [44.060385 N, -121.542268 W], 20.vii.1976, 1 male, USNM ENT 258064, George Steyskal (USNM); Whitman Natl. Fst. Anthony Lake, [44.958805 N, - 
118.23057 W], 2164m, 28.vii.1954, 1 female, OSAC 133387, J.H. Baker (OSAC); Anthony Lake, Blue Mts., [45.654334 N, -118.227668 W], 2438m, 4.viii.1929, 1 female, OSAC 133388, H.A. Scullen (OSAC); Whitman National Forest; Anthony Lake, 28.vii.1954, 1 male, JSS 23767, 3 females, JSS 23765, 23766, 23768, J.H. Baker (USNM); South Dakota: Spearfish Creek, Lawrence County, [44.589399 N, 103.886595 W], 14.vi.1969, 1 male, JSS 19476, W.W. Wirth (CNC); Hardy W.C. T3N, R1E, S30, 3.vii.1965, 1 male, CNCD 5445, R.W. Hodges (USNM); Utah: 24 Miles South of Hanksville, Henry Mountains, Garfield Co., [38.028415 N, -110.712679 W], 2286m, 29.vii.1968, 1 male, JSS 18295, J.E.H. Martin (CNC); Capitol Reef National Park, Wayne County, [38.177815 N, -111.176958 W], 3.x.1984, JSS 25137, W.P. Nye (EMUS); Mirror Lake, Duchesne County, [38.31073 N, -112.367492 W], 2743m, 21.vii.-9.viii.1977, JSS 25135, (EMUS); Mirror Lake, Duchesne County, [38.31073 N, 112.367492 W], 2.viii.1971, JSS 25138, G.F. Knowlton (EMUS); Willard Basin, Box Elder County, [41.394101 N, -111.973766 W], 2743m, 26.viii.1964, JSS 25134, W.J Hanson (EMUS); Tony Grove Creek, Logan, Cache County, [41.886599 N, 111.564102 W], 10.viii.1976, JSS 25136, (EMUS); Rt. 44, 2438m, 10.vii.1962, 1 female, JSS 21110, R.\&K. Dreisbach (MSUC); Cache Co., Green Canyon M.T., 31.viii.2003-25.viii.2003, 1 female, JSS 24611, W.J. Hanson \& Brammer (EMUS); Summit Co., Henry's Fork Pk., 2926m, 1.viii.1979-10.viii.1979, 1 female, CNCD 5807, S. \& J. Peck (CNC); Washington: Mount Adams, [46.204174 N, -121.493902 W], 3.viii.1930, 1 female, JSS 19460, F.P. Dean (CNC); Mt. Ranier; White River, [46.782709 N, -121.727952 W], 20.vii.1924, 1 male, JSS 23763, A.L. Melander (USNM); Spokane County, Buckeye, [47.842393 N,-117.374385 W], 21.vi.1930, 1 
male, JSS 19444, J.M. Aldrich (CNC); Spokane County, Mount Spokane, [47.923711 N, -117.111646 W], 2.vii.1930, 1 female, JSS 19447, J.M. Aldrich (CNC); Spokane County, Mount Spokane, [47.923711 N, -117.111646 W], 27.vi.1930, 1 female, JSS 19448, J.M. Aldrich (CNC); Spokane County, Mount Spokane, [47.923711 N, 117.111646 W], 22.vi.1930, 1 female, JSS 19457, J.M. Aldrich (CNC); Wawawai, 28.v.1922, 1 male, JSS 23762, A.L. Melander (USNM); Wyoming: Albany County, [41.727896 N, -105.684712 W], 2.viii.1927, 1 male, CNCD 26353, C.L. Corkins (CNC); Camp Guernsey Ryan Springs, [42.260113 N, -104.728624 W], 31.v.2005, 1 male, JSS 20686, B. Kondratieff, J. Schmidt, J. Owens (CSUC); Wyoming, [43.084281 N, -107.556417 W], 1952, 4 females, CNCD 26369-26372, F.M. Hull (CNC); Jet Lake, Yellowstone National Park, [44.550878 N, -110.808744 W], 16.viii.1927, 1 female, JSS 19439, J.M. Aldrich (CNC); Yellowstone Nat'l Park, Canyon Village, [44.587719 N, -110.537673 W], 21.vii.1971, 1 male, CNCD 5444, G.C. Steyskal (USNM); Canyon Campground, Yellowstone National Park, [44.733262 N, -110.486225 W], 12.viii.1918, 1 male, JSS 19461, 1 female, JSS 19462, A.L. Melander (CNC); Mount Washburn Yellowstone Park, [44.797716 N, -110.434368 W], 1 male, JSS 21343, C.T. Brues (MCZ); Snowy Range, 3048m, 8.ix.1950, 1 female, JSS 21105, R.R.D. \& R.K. Schwab (MSUC); Snowy Range, 23.viii.1951, 1 male, JSS 21108, 2 females, JSS 21106-21107, R.R. Dreisbach (MSUC); Centennial, 23.viii.1951, 2 female, JSS 21109, 21111, R.R. Dreisbach (MSUC); Centennial, Snowy Range, 3048m, 8.ix.1950, 1 female, JSS 21114, R.R.D. \& R.K. Schwab (MSUC).

\section{Dasysyrphus intrudens species complex (Osten Sacken)}


Figures: $6 \mathrm{~A}-\mathrm{F}, 17 \mathrm{~A} \& 19$

Synonyms and References:

Syrphus amalopis Osten Sacken, 1875: 148 (in part, description)

Syrphus intrudens 0sten Sacken, 1877: 326 (original description)

Syrphus amalopis Osten Sacken:

Williston, 1887: 69 (in part; redescription)

Syrphus disgregus Snow, 1895: 233 (original description; comb. nov.)

Curran, 1925: 180 (discussion)

Syrphus laticaudatus Curran, 1925: 176, Pl. XI Fig. 152 (original description; comb.

nov.)

Syrphus osburni Curran, 1925: 177, Pl. XI Fig. 153 (original description; comb. nov.) Metasyrphus amalopis (Osten Sacken):

Fluke, 1933: 110 (in part, includes disgregus and intrudens; key, redescription and transfer to Metasyrphus)

Metasyrphus laticaudatus (Curran):

Fluke, 1933: 111 (key, redescription and transfer to Metasyrphus)

Metasyrphus osburni (Curran):

Fluke, 1933: 112 (key, redescription and transfer to Metasyrphus) Dasysyrphus amalopis (Osten Sacken):

Stone et al., 1965: 563 (in part; includes intrudens, catalogue and move to Dasysyrphus] 
Dasysyrphus disgregus (Snow):

Stone et al., 1965: 563 (catalogue and move to Dasysyrphus) Dasysyrphus laticaudatus (Curran):

Stone et al., 1965: 563 (catalogue and move to Dasysyrphus) Dasysyrphus osburni (Curran):

Stone et al., 1965: 564 (catalogue and move to Dasysyrphus) Dasysyrphus venustus (Meigen):

Vockeroth, 1986: 203 (in part, includes disgregus, intrudens, laticaudatus and osburni; lectotype designation for disgregus and intrudens and discussion) Vockeroth, 1992: 70 (in part, includes intrudens, disgregus, laticaudatus and osburni; key and redescription)

Diagnosis: Abdominal maculae cross abdominal margin; maculae on tergite 2 large ( $>1 / 3$ width of tergite) oval-shaped; maculae on tergites $3 \& 4$ arcuate or lunulate, usually strongly constricted medially, sometimes dividing maculae in half, with medial edge swollen, never meeting medially, maculae reach abdominal margin, usually broadly contacting edge of abdomen (Figs $6 \mathrm{~A}-\mathrm{F}$ ). Has previously been confused with $D$. venustus, however, species in this complex have maculae that are arcuate, lunulate and/or greatly constricted medially, whereas in D. venustus they are more transverse and much less constricted medially (Fig. 8B).

Description: 
Body Length: $7.0-11.7 \mathrm{~mm}$; Wing Length: $7.0-10.7 \mathrm{~mm}$

Head: Frons dark with light pollenose fascia (in males it runs along ventral edge of where eyes meet, in females it is $1 / 4-1 / 2$ the length between the antennae and ocelli and narrowly to widely separated medially) and dark pile; face light with dark vitta $1 / 4-1 / 2$ the width of the face, usually reaching antennal socket, face with dark or light pile, sometimes with a both light and dark pile; gena dark with light pile; occiput dark, covered in light pollen, pile light; scape and pedicel light to dark, flagellomere usually light basally and darker apically, sometimes mostly dark.

Thorax: Scutum dark, sometime shiny or metallic, pile light; scutellum light with darker anterolateral edges, light pile some with varying amounts of dark pile posteriorly; cell $\mathrm{c}$ usually densely microtrichose but sometimes bare basally, $\mathrm{r}_{1}$ is usually bare at the base, but can be entirely microtrichose, br and bm range from densely microtrichose to extensively bare, but often with some bare areas, cup either densely microtrichose or bare at base and/or along CuP; haltere light; femora basally dark, apically light, tibiae light, pro- and mesotibiae sometimes with brown band toward apex, metatibia usually with brown band toward apex, tarsi usually dark anteriorly and light posteriorly, sometimes entirely light.

Abdomen: Maculae on tergite 2 large and oval, sometimes with anterolateral edge extending to edge of abdomen, maculae on tergites $3 \& 4$ arcuate or lunulate, usually strongly constricted medially, sometimes dividing maculae in half, with medial edge 
swollen, never meeting medially, maculae reach abdominal margin, usually broadly contacting edge of abdomen (Figs 6A-F); sternite 2 either all light, with dark oval macula or with dark fascia (usually distinct, sometimes faint), sternites $3 \& 4$ light with dark fasciae.

Male Genitalia: Surstylus more or less triangular in shape in lateral view, flattened anteroposteriorly with long pile on posterior side and spines on anteroventral side, similar to D. venustus (Fig. 17B) and D. limatus (Fig. 13B); cercus oval with long pile over entire surface; gonostylus is pointed ventrally, dorsally flattened at base then it expands dorsally towards apex, oblique ridge near apex, with ends of ridge projecting into points; pile on dorsal surface; basiphallus is basally bent at about a 90 degree angle towards the dorsum, at apical end it is curved ventrally, fully sclerotized all the way around with no pile or spines; distiphallus is long with no enlarged area basally, shaft sometimes with small bump dorsally near base, apical end flared out into horn with the dorsal side flattened slightly, which is mostly sclerotized but membranous at apex with setulae dorsally (Fig. 17A).

Intraspecific variation: The abdominal maculae are highly variable (Figs 6A-F), likely because multiple, unresolved species lie within this complex. Tergites $3 \& 4$ have maculae that can be arcuate or lunulate, usually strongly constricted medially, sometimes dividing maculae in half, medial edge swollen but degree of swelling varies, maculae usually broadly contact edge of abdomen, but in few specimens they end just past margin and do not contact edge; sternite 2 colouring variable, either 
all light, with dark oval macula or with dark fascia (usually distinct, sometimes faint); wing cells vary in microtrichia density with c usually densely microtrichose but sometimes bare basally, $r_{1}$ is usually bare at the base, but can be entirely microtrichose, br and bm range from densely microtrichose to extensively bare, but often with some bare areas, cup either densely microtrichose or bare at base and/or along CuP; distiphallus shaft sometimes with small bump dorsally near base.

Etymology: Presumably comes from the Latin, intrudo, meaning enter without invitation or permission.

Distribution: Canada (AB, BC, NF, NS, ON QC, YT) and US (AK, AZ, CA, CO, ID, MA, MD, ME, MI, NC, NH, NM, NY, OR, PA, TN, UT, VA, WA, WV, WY), also widespread over Europe and Asia (Fig. 19)

Ecology: Collected on great camas (Camassia leichtlinii (Baker) S. Watson, Liliaceae), common camas (Camassia quamash (Pursh) Greene, Liliaceae), Carolina spring beauty (Claytonia caroliniana Michaux, Portulacaceae), small-flowered woodlandstar (Lithophragma parviflorum (Hooker) Nuttall ex Torrey \& A. Gray , Saxifragaceae), heart-leaved foamflower (Tiarella cordifolia Linnaeus, Saxifragaceae), birch (Betula Linnaeus, Betulaceae), common cowparsnip (Heracleum maximum W. Bartram, Apiaceae), cowparsnip (Heracleum Linnaeus, Apiaceae), blackberry (Rubus Linnaeus, Rosaceae), Pacific blackberry (Rubus ursinus Chamisso \& Schlechtendal, Rasaceae), Pacific silver fir (Abies amabilis 
(Douglas ex Loudon) Douglas ex J. Forbes, Pinaceae), Labrador tea (Ledum

Linnaeus, Ericaceae), spotted-water hemlock (Cicuta maculata Linnaeus, Apiaceae), blueberry (Vaccinium Linnaeus, Ericaceae), dogwood (Cornus Linnaeus, Cornaceae), jack pine (Pinus banksiana Lambert, Pinaceae), mountain maple (Acer spicatum Lamarck, Aceraceae), nannyberry (Viburnum lentago Linnaeus, Caprifoliaceae), beachgrass (Ammophila Host, Poaceae), herb-Robert (Geranium robertianum Linnaeus, Geraniaceae), slender phlox (Microsteris gracilis (Hooker) Greene, Polemoniaceae), spreading groundsmoke (Gayophytum diffusum subsp. parviflorum F.H. Lewis \& Szweykowski, Onagraceae), chokecherry (Prunus virginiana, Rosaceae), bristly bearberry (Arctostaphylos columbiana Piper, Ericaceae), slender stitchwort (Minuartia tenella (J. Gay) Mattfeld, Caryophyllaceae), hairy cat's ear (Hypochaeris radicata Linnaeus, Asteraceae). Habitats listed on labels include "Ainus - Populus - Picea" or "Alder - Poplar - Spruce", "along creek in forested ravine", "along roadside", "Betula woods", "birch \& fir", "black spruce forest", "canopy gap in hardwood forest", "dry sand beach ridge", "gap in Mh/Be forest", "hardwood forest", "hemlock forest", "hot springs", "in clearing near river", "Ledum-Kalmia bog", "low vegetation and leaf litter at campsite", "marshy meadow", "on tide flats", "Picea forest", "pine forest", "Pinus-Salix forest", "riverbank scrub", "Salix-Picea", "scrub poplar and low vegetation", "seepage area", "fen", "sphagnum bog", "Vaccinium wood" and "wet, scrubby clearing". This species complex is also collected from hilltops. Collected January to November, although much more frequently collected in May to August. Collected from elevations from sea level $-4000 \mathrm{~m}$. 
Discussion: Dasysyrphus intrudens is most certainly a complex of multiple species. However, the boundaries between these species are still unclear. The COI clusters produced in the NJ tree (Fig. 4) yielded no clues to the number of species existing in this clade. When sorting the specimens by barcodes, no morphological patterns were visible. Sometimes characters were similar between two or more clusters and sometimes within one cluster there were multiple character states. As the barcoding region of COI appears to be of little help to sort this complex into species, other markers should be sequenced to look for insight into species boundaries. Fresh material collected into absolute alcohol will be required to sequence the necessary genes.

Note that this group has been referred to as the friuliensis group in conference abstracts, but never in a refereed publication. The oldest group name, intrudens, best serves as the name for the complex.

Type Material: "Lagunitas Creek, Cal./ April 15. O. Sacken”, "Osten/ Sacken/ Coll.”, red label "Type/ 875", yellow label "LECTOTYPE/ Syrphus/ intrudens 0.S./ Desig. Thompson 1977", "Syrphus/ intrudens 0.S.", J. Skevington/ Specimen \#/ 23783", male, deposited in the Museum of Comparative Zoology, Harvard University (MCZ). Both flagellomeres and right metaleg missing. Genitalia have been removed at some point and are not associated with the specimen (presumed to be lost). (examined)

Material Examined: Because this is a complex and species boundaries need to be 
resolved, it is of little use to list all information for material examined. The regions, specimen database numbers and depositions have been listed to make it easier to track these specimens for further study.

Canada: Alberta: CNCD 5818, 14511-14521, 14523-14536, 14539, 14541-14545, 106809, JSS 18347, 19367, 19368, 19525, 19526 (CNC); JSS 21072 (FAHS); JSS 25587 (WVSC); JSS 25659 (USNM); BOC 34679-34681 (ROME); UASM\# 149010149014, 149018-149022 (UASM); RBCM ENT 6006034 (RBCM); British Columbia: CNCD 5816, 5817, 7974, 8116, 8119, 8123, 14065-14080, 14084-14216, 14218$14222,14224-14226,14228-14230,14232-14241,14243-14250,14253-14283$, 14285-14288, 14290-14302, 14304-14313, 14315-14340, 14342-14357, 14359, 14361-14398, 14400-14404, 14406-14472, 14474-14508, 15381, 60084, 60086, 60089-60093, 60095-60099, 30904, 30905, 30907, 30910, 30911, JSS 18372, 19268, 19269, 19374, 19405, 19409-19411, 20781, 20827, 20828 (CNC); JSS $19995-19997,20000,20001,20003-20011,20013,20014,20016-20018,20020-$ $20022,20024,20026-20033,20036,20047,20062,20075-20086,20089-20091$, 20093, 20094, 20096-20107, 20109-20111, 20113, 20115-20121, 20124-20126, 20134, 20137, 20141-20145, 20147-20151, 20153-20156, 20158, 20161-20163, 20165, 20172, 20174, 20175, 20180, 20182, 20188, 20189, 20744 (UBCZ); JSS 20823, 20826 (RBCM); JSS 21308 (MCZ); JSS 23713 (CAS); JSS 25560-25570, 25572-25585 (WVSC); BOC 34682 (ROME); OSAC 133397, 133400, 133401, 133403, 133425, 133429, 133431, 133432, 133441 (OSAC); UASM 141937, 149009 (UASM); SFU 715497, 715565, 715868, 715931, 716133, 716181, 716481, 716544, $716878,716919,716995,716998,717335,717383,717389,717487,720561$ 
(SFUC); RBCM ENT 9881952, 9881953, 9881967, 9882348, 9882363, 9882364, $9911452,9911764,9915006,9915570,99166568,99166656,99166851$, 99188121, 99188123, 99188126-99188150 (RBCM); Manitoba: CNCD 14556, 14557 (CNC); JBWM 212507 (JBWM); New Brunswick: CNCD 14811-14814 (CNC); Newfoundland and Labrador: CNCD 14822-14825, JSS 18344 (CNC); Northwest Territories: CNCD 14037-14043, 14045, 14046, 14048-14051, 14054, 14055, 14057, 14059, 14061, 14063, 14064 (CNC); Nova Scotia: CNCD 14756, 14761, 14767, 14768, 14772, 14773 (CNC); Nunavut: CNCD 14044 (CNC); Ontario: CNCD $14558-14566,14568-14570,14577,14580,14581,14583,14584,14596,14597$, 14601-14605, 15387, 60347, 106800, JSS 19369, 19742, 21352, 21359-21370, 21372-21384, 21386-21393, 21395, 21396, 21398, 21399, 22443-22459, 24624, 24634-24636, 24720-24737, 24739-24749, 24780-24799, 24960-25001 (CNC); BOC 34662-34677, ROMEnt Spec. No. 57891, 74269, 98517, 98520, 98521, 98529, 98537-98539, 98542, 115134 (ROME); UASM\# 149017 (UASM); Quebec: LEM $8802,8809,8816,13604,13626,13685,13688,13843,13957$ (LEMQ); CNCD $14613-14645,14647-14650,14652,14655-14666,14668-14670,14673,14674$, $14676-14678,14682,14685,14687,14690,14691,14696-14710,14720,14723$, $14726-14735,14739-14744,14746-14752,14754,30877,30878,60060-60063$, 106801-106808, JSS 15331, 17748, 18343, 19675, 19725, 20760-20762, 20766, 20768, 20792-20794, 20800, 21317, 22101-22103, 24620 (CNC); JSS 25571 (WVSC); BOC 34678 (ROME); Saskatchewan: CNCD 14549 (CNC); Yukon Territory: CNCD 5814, 5815, 13910-13914, 13916-13948, 13950-13965, 13969$13973,13977-13980,13982-14004,14007-14009,14011-14028,14030-14036$, 
$60070,60071,60073,60074,60076,60078,106810$ (CNC); LEM 14302, 14304, 14466, 14469 (LEMQ); JSS 18369, 18377, 20769, 20771, 20772 (CNC); JSS 20073, 20133, 20157, 20160 (UBCZ); JSS 20817, 20818, 20821 (RBCM); ISS 23711, 23712, $23716,23720,23723,23739,23742,23749$ (CAS); United States of America: Alaska: JSS 18326-18329, 19272, 19338-19359, 19365, 19366, 19373, 19378, 19383-19389, 19403, 19406, 19528-19531, 19550, 19555, 19556, 20775-20777, 20779 (CNC); ISS 20136 (UBCZ); JSS 21298, 21313 (MCZ); JSS 23715, 23717, $23726,23727,23733,23738,23754,23756-23759$ (CAS); ISS 25642 (USNM); CNCD $26551,26552,26554-26573,26576,26580-26592,30879,30880,30891,30892$, 30894, 30900, 30901, 60085 (CNC); OSAC 133392, 133404, 133433, 133455, 133458 (OSAC); Arizona: JSS 25553 (WVSC); CNCD 26636 (CNC); OSAC 133384 (OSAC); USNM ENT 258088 (USNM); California: JSS 18332, 18333, 18337, 19270, 19408, 19706, 20791 (CNC); JSS 20576-20579, 20581-20583, 20591, 20592, 20612, 20619, 20621, 20624 (EMEC); ISS 20952, 20953, 20959-20961, 20964, 20965, 20973, 20990, 20991, 20994 (MHPC); JSS 21071 (CDFA); JSS 21303, 21304, 21312 (MCZ); JSS 23725, 23731, 23734, 23740, 23745, 23753, 23881, 23882, 23884, 23891, 23896, 23899, 23903 (CAS); JSS 25070 (EMUS); JSS 25639, 25643, 25649, 25650, 25658 (USNM); CNCD 26637-26648, 26686-26708 (CNC); Colorado: CNCD 5441, 5455, 26606-26635, 26660-26684, 60031, 60032, JSS 19260 , $19261,19331,19375,19376,19382,19400,19434,19567,23700-23703,23705$, 24637, JSS 20634-20658, 20660, 20661, 20663, 20664, 20666, 20667, 20687, 20688, 20691, 20731, 20832 (CSUC); ISS 20815 (FMNH); ISS 21104 (MSUC); JSS 21130-21133, 21294 (MCZ); JSS 23704, 24621 (DEBU); JSS 25551, 25552, 25554, 
25557 (WVSC); USNM ENT 258075 (USNM); Connecticut: JSS 19569, 19570, 19573-19576 (CNC); Idaho: JSS 19266, 19267, 19271, 19274, 19285, 19286, 19362-19364, 19370, 19379, 19380, 19391-19395, 19407, 19429, 19430, 19527, 19532, 19566 (CNC); JSS 20617 (EMEC); JSS 21150 (MSUC); ISS 21154, 21302, 21311 (MCZ); JSS 25062, 25073 (EMUS); JSS 25635, 25653-25656 (USNM); CNCD 26603, 26604, 26654 (CNC); RBCM ENT 99188125 (RBCM); Maine: LEM 8808 (LEMQ); JSS 19662, 19663, 19674 (CNC); Maryland: CNCD 26727, 26729-26731 (CNC); Massachusetts: JSS 19428, 19548, 19571, 19577, 19578 (CNC); ISS 21322, 21327 (MCZ); JSS 23755, 23906 (CAS); USNM ENT 258061, 258071 (USNM); Michigan: JSS 21098, 21099, 21120-21128, 21135, JSS 21137 (MSUC); Missouri: JSS 25558, 25559 (WVSC); Montana: JSS 19273, 19401, 19426, 19463 (CNC); JSS 25652 (USNM); CNCD 26594, 26605 (CNC); Nevada: JSS 19381 (CNC); JSS 24738 (EMUS); New Hampshire: USNM ENT 258089, 258096-258104 (USNM); CNCD $5439,5440,5446,5447,5451,26723,26724,26732,26736$, JSS 19523, 1964019661, 19664, 19665 (CNC); JSS 21295, 21301 (MCZ); New Mexico: JSS 18334, 18345, 19568 (CNC); JSS 19982 (FMNH); JSS 20989, 20993 (MHPC); ISS 2134521347 (MCZ); CNCD 26517 (CNC); New York: JSS 19249-19252, 19259 (FMNH); JSS 19673 (CNC); JSS 21155, 21289, 21291-21293, 21296, 21297, 21307, 21316, 21344 (MCZ); CNCD 26725, 26737, 26738, 26744 (CNC); USNM ENT 258095 (USNM); North Carolina: JSS 19666-19672 (CNC); JSS 20982, 20984-20986 (MHPC); CNCD 26726 (CNC); Oregon: JSS 18330, 18331, 18335, 19275, 19279 19284, 19399, 19402, 19549, 20812, 20813 (CNC); JSS 20615, 20618, 20631 (EMEC); JSS 24641 (DEBU); JSS 25555, 25556 (WVSC); JSS 25646-25648 (USNM); 
CNCD 265952665126658 (CNC); OSAC 133406, 133409, 133411-133413, $133415,133416,133437,133454,133456,133457,133459$ (OSAC); USNM ENT 258060 (USNM); Pennsylvania: USNM ENT 258079, 258080, 258082-258087 (USNM); Tennessee: JSS 20798, 20799 (CNC); Utah: JSS 19390, 19404, 19427 (CNC); JSS 21129, 21134 (MSUC); JSS 23890 (CAS); JSS 24619, 24628-24633, $25004,25010-25022,25024-25039,25041-25061,25063-25069,25071,25072$, 25074-25079, 25110-25120 (EMUS); JSS 25634 (USNM); CNCD 26659, 26714, 26715-26722, 60036, 60041 (CNC); USNM ENT 258078 (USNM); Virginia: JSS 18340 (CNC); CNCD 26733 (CNC); USNM ENT 258076 (USNM); Washington: CNCD $5462,26593,26596-26602,26649,26650,26652,26653,26655-26657,60044$, $60045,60048-60053,60055-60057,60059$, JSS 19262-19265, 19276-19278, 19287-19330, 19332-19336, 19360, 19361, 19371, 19372, 19377, 19396-19398, 19416-19425, 19469, 20811, 23707, 24622, 24627, 24639, 24640 (CNC); ISS 20608-20611, 20614, 20616 (EMEC); JSS 20689, 20690, 20803 (CSUC); JSS 20738, 20740 (LEMQ); JSS 21100, 21101 (MSUC); JSS 21306, 21309, 21310 (MCZ); JSS $23730,23735,23737,23744,23746,23885,23892,23895,23898,23901,23902$, 23909 (CAS); JSS 24623, JSS 24638 (DEBU); JSS 25636-25638, 25640, 25641, 25644, 25645, JSS 25657 (USNM); OSAC 133393-133396, 133398, 133399, 133402, $133405,133407,133408,133410,133414,133417-133424,133426,133428$, $133430,133434-133436,133438-133440,133442,133443,133445-133453$ (OSAC); USNM ENT 258090, 258094 (USNM); West Virginia: CNCD 5454 (USNM); USNM ENT 258092 (USNM); Wisconsin: JSS 21326 (MCZ); Wyoming: ISS 19254 (FMNH); JSS 19412-19415 (CNC); JSS 21102 (MSUC); JSS 21156, 21315, 21321, 
21323-21325 (MCZ); JSS 25023 (EMUS); JSS 25651 (USNM); CNCD 26685, 26709, 26713 (CNC).

\section{Dasysyrphus laticaudus (Curran)}

Figures: 7D-F, 15A \& 20

Synonyms and References:

Syrphus laticaudus Curran, 1925: 175, Pl. XI Fig. 151 (original description) Metasyrphus laticaudus:

Fluke, 1933: 117 (key, redescription and transfer to Metasyrphus) Dasysyrphus laticaudus:

Stone et al., 1965: 563 (catalogue and transfer to Dasysyrphus)

Vockeroth, 1986: 203 (synonymized under pauxillus)

Vockeroth, 1992: 68 (key and redescription)

Diagnosis: Maculae oblique and fairly uniform in thickness. Like D. pauxillus, $D$. pacificus and D. nigricornis, maculae on tergites $3 \& 4$ do not reach edge of abdominal margin (Figs 7D-F). Dasysyrphus laticaudus differs from the other three in that the markings on tergite 2 in the female extend anterolaterally to the edge of the abdomen (Fig. 7D) and in the male they extend similarly, sometimes only slightly, but usually do not reach the edge (Fig. 7F). Maculae on tergites $3 \& 4$ usually come 
very close to the margin, if not narrowly touching it, but never crossing it (Figs 7DF).

Description:

Body Length: $5.0-8.2 \mathrm{~mm}$; Wing Length: $4.8-7.8 \mathrm{~mm}$

Head: Frons dark with light pollenose fascia (in males it runs along ventral edge of where eyes meet, in females it is $1 / 3-1 / 2$ the length between the antennae and ocelli with a separation medially and edges triangular to rounded) and dark pile, some females with light pile; face light with dark vitta $1 / 4-1 / 3$ the width of the face, usually coming close but not reaching antennal socket, face with dark pile lateral to the eye, shorter in females; gena dark with light to dark pile; occiput dark, covered in light pollen, pile light; scape and pedicel light to dark, flagellomere usually dark with light base.

Thorax: Scutum dark, sometime shiny or metallic, pile variable, light to dark; scutellum light to dark with darker lateral edges, pile on female mostly light with some dark, male typically with dark pile, but sometimes with some light pile anteriorly; wings densely microtrichose, some with small bare areas in br above the base of the spurious vein; haltere light; femora of male dark basally, apically light, of female light (Fig. 7D), sometimes with dark base, tibiae light, metatibia sometimes 
with dark band close to apex, tarsi light with darker anterior side, mesotarsus of female light.

Abdomen: Maculae on tergite 2 large and oval, in female the anterolateral edge projects to contact the edge of the tergite (Fig. 7D), in males there is a small projection but it terminates in a point before the edge (Fig. 7F), maculae on tergites $3 \& 4$ are oblique fairly straight and of uniform thickness, maculae do not reach abdominal margin (Figs 7D-F); sternites light with dark fasciae of varying thickness.

Male Genitalia: Surstylus more or less triangular in lateral view, flattened posteriorly, long pile on dorsal, laterodorsal and posterodorsal sides, row of spines on posteriomedial edge; cercus oval with long pile over entire surface; gonostylus boot-shaped but slightly more narrow apically than basally, with projection on posterodorsal surface, projecting dorsally, pile on dorsal surface; basiphallus elongate, basally bent at a slightly more than 90 degree angle towards dorsum, apical end curved ventrally, but does not greatly extend ventrally and is partly membranous dorsally, with spines on posterodorsal side; distiphallus elongate, apical end flared out into horn, which is mostly sclerotized but membranous at apex, with no setulae dorsally, basally bent towards dorsum and usually greatly enlarged at bend with a triangular projection, projecting ventrally (Fig. 15A). Extremely similar to D. pauxillus (Fig. 16A). 
Intraspecific Variation: Female maculae on tergite 2 tend to be similar across individuals, however on males, the extent of projection on the anterolateral edge can vary. Male femora are always dark basally and light apically. Many females have predominantly light femora (especially the profemur; Fig. 7D). Females from the northwestern part of the range (Yukon) have femora that are dark basally and light apically. Wing cells are usually densely microtrichose, but sometimes have very small bare areas in cell br, above the spurious vein. Basal horn of distiphallus occasionally has few minute setulae dorsally.

Etymology: Comes from the Latin words latus, meaning broad and cauda, meaning tail.

Distribution: Eastern and northern Canada (MB, NB, NT, NS, NU, ON, QC, YK) and northeastern and northern western USA (AK, CT, ME, MA, MI, NH, NY). (Fig. 20)

Ecology: Collected from April - July, but more commonly in June and July. Collected at elevations of $792-1067 \mathrm{~m}$. Habitats this species has been collected from, according to label data, are "Mh/Be forest" (mixed hardwood/beech), "hardwood forest", "mixed forest", "pine forest", "coniferous woods", "at top of limestone bluff" and "top of open S-facing bluff". It has been recorded as being collected on pine trees (Pinus Linnaeus; Pinaceae).

Discussion: Dasysyrphus laticaudus is hereby resurrected from synonymy with 
Dasysyrphus pauxillus (synonymized by Vockeroth (1986)). Vockeroth (1986) considered them to be the same species, presumably with only slight morphological differences between the species being mistaken for intraspecific variation.

Dasysyrphus laticaudus is very similar to pauxillus (Figs 11A, B) and pacificus (Figs 10D, E), with the later two being extremely difficult to distinguish from each other. There are however, morphological and genetic characters that distinguish laticaudus from pauxillus and pacificus. Their distributions are also predictable and largely non-overlapping.

Type Material: “Orillia Ont/ 5.v.1921", “Collector/ H. Curran”, red label "HoloTYPE/ Syrphus/ laticaudus/ CNC No. 497 Curr.", "CNC Diptera/ \#15382"; male, deposited in the Canadian Collection of Insects, Arachnids and Nematodes (CNC), Ottawa, ON. (examined)

Material Examined:

Paratypes: Canada: Ontario: Orillia, Simcoe Co., [44.609505 N, -79.42068 W], 4.v.1921, 1 female, CNCD 13851, H. Curran (CNC); Orillia, Simcoe Co., [44.609505 N, -79.42068 W], 5.v.1921, 1 male, CNCD 13885, H. Curran (CNC); Other Material Examined: Canada: Manitoba: Winnipeg, [49.88333333 N, $-97.13333333 \mathrm{~W}]$, 20.vii.1948, 1 male, JBWM 206845, W.H. Fell (JBWM); Winnipeg, [49.88333333 N, 97.13333333 W], 24.v.1949, 1 female, JBWM 206517, C.F. Barrett (JBWM); New Brunswick: Charlotte County, [45.23102 N, -66.925304 W], 9.vi.1955, 1 female, CNCD 13909, W.T.A. Neilson (CNC); York County, Fredericton, [45.959225 N, - 
66.640351 W], 3.v.1913, 1 male, CNCD 13900, (CNC); York County, Fredericton, Neville's Field, [45.959225 N, -66.640351 W], 5.vi.1958, 1 male, CNCD 13901, (CNC); Aboushagan Rd., 10km N Sackville, [45.998 N, -64.3591 W], 7.v.2010, 1 male, JK 969, J. Klymko, S.L. Robinson, D.M.Mazerolle (NBMB); Kouchibouguac National Park, Kent Co., [46.819201 N, -64.96788 W], 19.v.1977, 1 male, JSS 18365, W.P. Hanley (CNC); Kouchibouguac National Park, Kent Co., [46.819201 N, 64.96788 W], 20.v.1977, 1 male, CNCD 13897, B. E. Cooper (CNC); Kouchibouguac National Park, Kent Co., [46.819201 N, -64.96788 W], 22.v.1977, 1 male, CNCD 13899, 2 females, CNCD 13905, 13906, Hanley \& Cooper (CNC); Kouchibouguac National Park, Kent Co., [46.819201 N, -64.96788 W], 23.v.1977, 1 male, JSS 18364, J.D. Lafontaine (CNC); Kouchibouguac N.P., 23.v.1977, 1 male, CNCD 106793, Hanley \& Cooper (CNC); Kouchibouguac National Park, Kent Co., [46.819201 N, -64.96788 W], 24.v.1977, 1 male, CNCD 13898, 1 female, CNCD 13907, B. E. Cooper (CNC); Kouchibouguac National Park, Kent Co., [46.819201 N, -64.96788 W], 26.v.1977, 1 female, CNCD 13908, W.P. Hanley (CNC); Northwest Territories: Wholdaia Lake, [60.680702 N, -104.293128 W], 2.vii.-4.vii.1966, 1 female, CNCD 13779, J.G. Chillcott (CNC); 21 miles east Tuktoyaktuk, [69.426844 N, -132.172475 W], 20.vi.197125.vi.1971, 1 female, CNCD 13773, D.M. Wood (CNC); Nova Scotia: Cape Breton Highlands National Park, Pleasant Bay, [46.73333333 N, -60.63333333 W], 9.vi.1984, CNCD 60066, B.E. Cooper (CNC); Inverness Co., Cape Breton Highlands National Park, Pleasant Bay, [46.822961 N, -60.799065 W], 12.vi.1984, 1 female, CNCD 13904, B.E. Cooper (CNC); Nunavut: Ford Lake, M.T.S. Gravity Survey Camp, [63.133386 N, -107.416561 W], 28.vi.1966, 3 males, CNCD 13716-13718, G.E. 
Shewell (CNC); Ontario: Fenolon Falls, Kawartha Lakes Div., [44.538149 N, 78.735433 W], 27.v.1927, 1 female, CNCD 13856, F.P. Ide (CNC); Chaffeys Locks area, [44.56666667 N, -76.31666667 W], 15.v.1975, 1 male, ROMEnt Spec. No. 115136, J.C.E. Riotte (ROME); Maynooth, Hastings Co., [45.229716 N, -77.940949 W], 25.v.1951, 1 female, CNCD 13857, J.F. McAlpine (CNC); Maynooth, Hastings Co., [45.229716 N, -77.940949 W], 22.v.1970, 2 males, CNCD 13883, 13884, D.M. Wood (CNC); Hastings County, Maynooth, [45.229716 N, -77.940949 W], 10.v.1987, CNCD 60346, D. Bell \& M. Wood (CNC); Griffith, 7 miles East, [45.243279 N, -77.031193 W], 31.v.1983, 1 female, CNCD 13858, B.E. Cooper (CNC); By Cranjelly Lake, Algonquin Provincial Park, [45.28719 N, -78.28012 W], 7.v.-21.v.2009, JSS 22433, 22435, E. Proctor (CNC); By Cranjelly Lake, Algonquin Provincial Park, [45.28719 N, -78.28012 W], 21.v.-4.vi.2009, JSS 22436, E. Proctor (CNC); Ottawa, Stack Road, Sand, [45.320852 N, -75.736188 W], 7.v.2007, 6 males, CNCD 30882-30887, J.R. Vockeroth (CNC); By Madawaska Lake, Algonquin Provincial Park, [45.3261 N, 78.3056 W], 12.v.-27.v.2007, 2 male, JSS 20747, 21358, E. Proctor (CNC); By Madawaska Lake, Algonquin Provincial Park, [45.32658 N, -78.30506 W], 9.vi.24.vi.2008, JSS 22430, 24717, 24718, E. Proctor (CNC); By Madawaska Lake, Algonquin Provincial Park, [45.32658 N, -78.30506 W], 15.v.-29.v.2009, JSS 22434, E. Proctor (CNC); By Madawaska Lake, Algonquin Provincial Park, [45.32936 N, 78.30364 W], 7.vi.-21.vi.2008, JSS 24719, E. Proctor (CNC); By Madawaska Lake, Algonquin Provincial Park, [45.32936 N, -78.30364 W], 1.v.-15.v.2009, JSS 22431, E. Proctor (CNC); Algonquin Park, By Crossbar Lake, [45.32686 N, -78.29997 W], 15.v.29.v.2009, 1 male, JSS 24614, 22432, E. Proctor (CNC); By Florence L. Algonquin P.P. 
[Florence Lake, Algonquin Provincial Park], [45.44371 N, -78.49012 W], 13.v.29.v.2007, 1 male, JSS 21353, E. Proctor (CNC); By Florence Lake, Algonquin Provincial Park, [45.44371 N, -78.49012 W], 19.v.-3.vi.2009, JSS 22437, E. Proctor (CNC); By Sitting Duck Lake, Algonquin Provincial Park, [45.44985 N, -78.46831 W], 21.v.-21.v.2008, JSS 24643, E. Proctor (CNC); By Cecil Lake, Algonquin Provincial Park, [45.4511 N, -78.4956 W], 6.vi.-20.vi.2008, JSS 25250, E. Proctor (CNC); By Pondweed Lake, Algonquin Provinical Park, [45.46488 N, -78.43066 W], 6.vi.19.vi.2008, ISS 23709, E. Proctor (CNC); By Pondweed Lake, Algonquin Provinical Park, [45.46552 N, -78.42949 W], 26.v.-9.vi.2009, JSS 22441, E. Proctor (CNC); 2 km E of Pondweed L. [Lake], Algonquin Provincial Park, [45.4654 N, -78.4298 W], 16.v.28.v.2007, 2 females, JSS 21354, 21355, E. Proctor (CNC); 2 km E of Pondweed L. [Lake], Algonquin Provincial Park, [45.4654 N, -78.4298 W], 9.v.-16.v.2007, 2 males, JSS 21356, 21357, E. Proctor (CNC); Constance Bay, meadow, [45.495933 N, 76.08694444 W], 18.iv.2010, 1 male, JSS 19757, J. Skevington (CNC); Algonquin Park, By Lake of Two Rivers, [45.57177 N, -78.46515 W], 11.v.-25.v.2009, 1 male, JSS 24613, E. Proctor (CNC); By Lake of Two Rivers, Algonquin Provincial Park, [45.57177 N, -78.46515 W], 11.v.2009, JSS 22440, E. Proctor (CNC); By Lake of Two Rivers, Algonquin Provincial Park, [45.57219 N, -78.46486 W], 11.v.200925.v.2009, JSS 22438, 22439, E. Proctor (CNC); Algonquin [Provincial] Park, Kearney Lake Campround, [45.57267 N, -78.439397 W], 11.vi.2005, 1 female, JSS 19761, L. Bartels (CNC); Moose Pens, L. Sasajewun, Algonquin Pk., [45.58 N, -78.36 W], 19.v.1965, 1 male, ROMEnt Spec. No. 115125, S.M. Smith (ROME); By Brewer Lake, Algonquin Provincial Park, [45.58551 N, -78.31044 W], 18.v.2009-2.vi.2009, 
JSS 22442, E. Proctor (CNC); Algonquin Park, [45.86666667 N, -78.38333333 W], 11.vi.2003-18.vi.2003, 1 male, ROMEnt Spec. No. 98519, 1 female, ROMEnt Spec. No. 98518, Mark Vanderwel (ROME); Low Bush, Lake Abitibi, Cochrane District, [48.91608 N, -80.139852 W], 7.vi.1925, 1 female, CNCD 13852, N.K. Bigelow (CNC); Low Bush, Lake Abitibi, Cochrane District, [48.91608 N, -80.139852 W], 9.vi.1925, 1 female, CNCD 13853, N.K. Bigelow (CNC); Low Bush, Lake Abitibi, Cochrane District, [48.91608 N, -80.139852 W], 14.vi.1925, 1 female, CNCD 13854, N.K. Bigelow (CNC); Low Bush, Lake Abitibi, Cochrane District, [48.91608 N, -80.139852 W], 16.vi.1925, 1 female, CNCD 13855, N.K. Bigelow (CNC); LowBush, Lake Abitibi, [48.91608 N, -80.139852 W], 9.vi.1923, UASM\# 149023, N.K. Bigelow (UASM); LowBush, Lake Abitibi, [48.91608 N, -80.139852 W], 6.vi.1925, BOC 34683, N.K. Bigelow (ROME); LowBush, Lake Abitibi, [48.91608 N, -80.139852 W], 7.vi.1925, 1 female, BOC 34684, N.K. Bigelow (ROME); LowBush, Lake Abitibi, [48.91608 N, 80.139852 W], 9.vi.1925, 2 females, BOC 34685, 34686, N.K. Bigelow (ROME); LowBush, Lake Abitibi, [48.91608 N, -80.139852 W], 13.vi.1925, 1 female, BOC 34687, N.K. Bigelow (ROME); LowBush, Lake Abitibi, [48.91608 N, -80.139852 W], 16.vi.1925, 2 females, BOC 34688, 34689, N.K. Bigelow (ROME); LowBush, Lake Abitibi, [48.91608 N, -80.139852 W], 24.vi.1925, 1 female, BOC 34690, N.K. Bigelow (ROME); Low Bush, Lake Abitibi, [48.91608 N, -80.139852 W], 14.vi.1925, 1 female, JSS 21337, N.K. Bigelow (MCZ); Low Bush, Lake Abitibi, [48.91608 N, -80.139852 W], 15.vi.1925, 1 female, JSS 21338, N.K. Bigelow (MCZ); Almonte, 18.v.1951, 1 male, CNCD 106791, J.F. McAlpine (CNC); Quebec: Aylmer, [45.400224 N, 75.817137 W], 28.v.1923, 2 females, CNCD 13866, 13867, C.H. Curran (CNC); 
Aylmer, [45.400224 N, -75.817137 W], 29.v.1923, 1 female, CNCD 13868, C.H. Curran (CNC); Aylmer, [45.400224 N, -75.817137 W], 16.v.1924, 5 males, CNCD 13887-13890, 106792, 2 females, CNCD 13864, 13865, C.H. Curran (CNC); Aylmer, [45.400224 N, -75.817137 W], 10.v.1925, 1 male, CNCD 13886, C.H. Curran (CNC); Hull, Communaute-Urbaine-de-l'Outaouais, [45.447639 N, -75.733192 W], 26.v.1923, 4 females, CNCD 13859-13862, C.H. Curran (CNC); Hull, CommunauteUrbaine-de-l'Outaouais, [45.447639 N, -75.733192 W], Hull, Communaute-Urbainede-l'Outaouais, [45.447639 N, -75.733192 W], 24.v.1923, 1 female, CNCD 13863, C.H. Curran (CNC); Hull, Communaute-Urbaine-de-l'Outaouais, [45.447639 N, 75.733192 W], 14.v.1924, 2 males, CNCD 13891, 13892, C.H. Curran (CNC); Rigaud, Summit of Mount Rigaud, Vaudreuil-Soulanges, [45.466534 N, -74.325875 W], 13.v.2008, 1 male, JSS 17647, J. Skevington, J. Gibson, A. Sugarman (CNC); Rigaud, Summit of Mount Rigaud, Vaudreuil-Soulanges, [45.466534 N, -74.325875 W], 1.v.1986, 1 male, JSS 18342, B.E. Cooper (CNC); Summit Mtn. Rigaud, [45.466534 N, -74.325875 W], 10.v.1986, 3 males, JSS 20763-20765, B.E. Cooper (CNC); Hull, [45.5 N, -75.83333333 W], 27.v.1923, 1 female, BOC 34691, C.H. Curran (ROME); Beechgrove, Les Collines-de-l'Outaouais, [45.64508 N, -76.123602 W], 15.v.1951, 1 female, CNCD 13874, J.F. McAlpine (CNC); Beechgrove, Les Collines-de-l'Outaouais, [45.64508 N, -76.123602 W], 3.v.1968, 1 male, CNCD 13894, 1 female, CNCD 13875, J.F. McAlpine (CNC); Beechgrove, Les Collines-de-l'Outaouais, [45.64508 N, 76.123602 W], 27.v.1963, 1 female, CNCD 13876, J.R. Vockeroth (CNC); Beechgrove, Les Collines-de-l'Outaouais, [45.64508 N, -76.123602 W], 15.v.1961, 1 male, CNCD 13895, 1 female, CNCD 13877, J.R. Vockeroth (CNC); Duncan Lake, near Rupert, 
[45.68138889 N, -76.05027778 W], 5.vi.1971, 1 female, CNCD 13881, J.F. McAlpine (CNC); Lac Mondor, Near Ste. Flore, Le Centre-de-la-Maurice, [46.625254 N, 72.768842 W], 15.v.1951, 1 female, CNCD 13869, E.G. Munroe (CNC); Lac Mondor, Near Ste. Flore, Le Centre-de-la-Maurice, [46.625254 N, -72.768842 W], 16.v.1951, 1 male, CNCD 13893, 1 female, CNCD 13870, E.G. Munroe (CNC); Lac Mondor, Near Ste. Flore, Le Centre-de-la-Maurice, [46.625254 N, -72.768842 W], 21.v.1951, 2 females, CNCD 13871, 13872, E.G. Munroe (CNC); Mont Albert, La Haute-Gaspesie, [49.131813 N, -66.464367 W], 1067m, 1.vii.1954, 1 male, CNCD 13896, W.J. Brown (CNC); Rupert River, [51.483333 N, -78.766667 W], 19.vi.1956, 1 female, CNCD 13878, J.R. Lonsway (CNC); 37 km North West of Schefferville, Sept-RivieresCaniapiscau, [55.038335 N, -66.424521 W], 12.vi.1981, 1 female, CNCD 13880, F. Brodo (CNC); Great Whale River, [55.116816 N, -76.405554 W], 7.vii.1949, 1 female, CNCD 13882, J.R. Vockeroth (CNC); Yukon Territory: 10 km North of Carcross on Highway 2, [60.2596 N, -134.749909 W], 5.vi.1981, 1 female, JSS 18373, C.S. Guppy (CNC); Bluefish Ridge, [67.15 N, -140.6166667 W], 792m, 2.vii.1983, 2 females, JSS 20049, 20050, R.J. Cannings (UBCZ); Bluefish Ridge, [67.15 N, -140.6166667 W], 792m, 7.vii.1983, 1 female, JSS 20069, R.J. Cannings (UBCZ); 6 km East of Old Crow, [67.566667 N, -139.683333 W], 13.vii.1981, 1 female, JSS 18366, C.S. Guppy (CNC); Old Crow, [67.571219 N, -139.833877 W], 4.vii.1983, JSS 20071, R.A. Cannings (UBCZ); Old Crow, [67.61666667 N, -139.25 W], 13.vii.1981, CNCD 60075, C.S. Guppy (CNC); Firth River, [69.207056 N, -140.071033 W], 22.vi.1984, 1 female, JSS 18375, S.G. Cannings (CNC); United States of America: Alaska : Matanuska-Susitna County, Matanuska, [61.541945 N, -149.229674 W], 12.vi.1944, 1 female, JSS 19515, 
J. Chamberlin (CNC); Connecticut: New London County, Lyme, [41.400831 N, 72.342951 W], 4.v.1918, 1 male, JSS 19491, C.T. Greene (CNC); Maine: Crystal, Aroostook County, [45.95953 N, -68.361018 W], vi.1980, 1 male, CNCD 26441, S.N. Pease (CNC); Maryland: Prince George's County, Laurel, [39.101761 N, -76.860489 W], 25.v.1965, 1 male, CNCD 26728, (CNC); Massachusetts: Worcester County, Sunderland, [42.244545 N, -71.77175 W], 15.v.1965, 1 male, JSS 19492, F.C. Thompson (CNC); Hampshire County, Amherst, [42.34038 N, -72.496818 W], 3.v.1963, 4 males, JSS 19493,-19496, F.C. Thompson (CNC); Amherst, Hampshire County, [42.34038 N, -72.496818 W], 7.v.1963, 1 male, JSS 19497, F.C. Thompson (CNC); Amherst, Hampshire County, [42.34038 N, -72.496818 W], 14.v.1963, 1 male, JSS 19498, F.C. Thompson (CNC); Michigan: Crawford County, 27.v.1958, 1 female, JSS 21151, 21152, R. \& K. Dreisbach (MSUC); New Hampshire: Cheshire County, Jaffrey, [42.814957 N, -72.022399 W], 4.vi.1919, 1 female, CNCD 26424, C.W. Johnson (CNC); Glen House, [44.289163 N, -71.225764 W], 14.vi.1911, 1 female, JSS 21339, N.K. Bigelow (MCZ); Mt. Monadnock, 11.vi.1932, 1 female, JSS 23773, A.L. Melander (USNM); New York: Ithaca, Tompkins County, [42.444589 N, 76.499268 W], 11.v.1898, 1 female, CNCD 26425, C.W. Johnson (CNC); Essex County, Corlear Bay, Lake Champlain, [44.483961 N, -73.418207 W], vi.1939, 1 female, JSS 19499, R.C. Shannon (CNC).

\section{Dasysyrphus limatus (Hine)}

Figures: 8C, D, 13B \& 21 
Synonyms and References:

Syrphus limatus Hine, 1922: 146 (original description)

Curran, 1925: 178, Pl. XI Fig. 154 (redescription)

Metasyrphus limatus:

Fluke, 1933: 114 (key, redescription and transfer to Metasyrphus)

Dasysyrphus limatus:

Stone et al., 1965: 563 (catalogue and transfer to Dasysyrphus)

Vockeroth, 1986: 202 (lectotype designation)

Vockeroth, 1992: 66 (key and redescription)

Diagnosis: Abdominal maculae cross abdominal margin; maculae on tergite 2 very small oval-shaped; maculae on tergites $3 \& 4$ transverse, narrow and slightly widened medially (Fig. 8C). Similar to D. venustus (Fig. 8B) however the maculae on tergite 2 are always small in $D$. limatus, less than $1 / 4$ the width of the tergite, and are much larger in $D$. venustus, greater than $1 / 3$ the width of the tergite.

Description:

Body Length: $7.5-10.9 \mathrm{~mm}$; Wing Length: $6.8-10.4 \mathrm{~mm}$

Head: Frons dark with light pollenose fascia (in males it runs along ventral edge of where eyes meet, in females it is $1 / 2$ or less the length between the antennae and 
ocelli, with a distinct separation medially) and dark pile; face light with dark vitta $1 / 3-1 / 2$ the width of the face and reaches the antennal socket, face with dark pile, bare medially; gena dark with some light pollen ventral to eye, light and dark pile; occiput dark, covered in light pollen, pile light, some specimens with some dark pile; scape, pedicel and flagellomere dark.

Thorax: Scutum dark, sometime shiny or metallic, pile dark in males and light in females; scutellum light to dark with darker lateral edges, pile dark with some light pile along lateral edges to light with some dark on posterior edge; wing cell br bare at base, above spurious vein, $\mathrm{bm}$ bare on anterior half, $\mathrm{r}_{1}$ bare at base and some with cup bare posterior to vein CuP; haltere light; femora basally dark, apically light, metafemur light only at apex, pro- and mesotibiae light, metatibia dark, light at proximal end, pro- and mesotarsi light with darker anterior side, metatarsi dark.

Abdomen: Maculae on tergite 2 small, slender and oval, $<1 / 4$ width of tergite, maculae on tergites $3 \& 4$ are transverse, slender and widen medially, positioned anteriorly on the tergite, maculae reach abdominal margin (Fig. 8C); sternites light with thick, dark fasciae.

Male Genitalia: Surstylus more or less oval in shape in lateral view, flattened with long pile on posterior side and spines on anteroventral side; cercus oval with long pile over entire surface; gonostylus triangular-shaped in lateral view, wide at base and elongated posterodorsal with small pointed projection on posterodorsal edge, 
posterior edge flares laterally, making it difficult to see in lateral view; pile on dorsal surface; basiphallus is greatly enlarged dorsally, fully sclerotized all the way around with no pile or spines; distiphallus is short with no enlarged area basally, apical end flared out into horn, which is mostly sclerotized but membranous at apex with no setulae dorsally (Fig. 13B).

Intraspecific variation: Maculae on abdomen can be of varying thickness, however they are usually quite narrow.

Etymology: The word limatus is Latin, meaning polished, refined.

Distribution: Canada (AB, BC, NT, NS, QC, YT), USA (AK, CO, MA, ME, NY, UT) (Fig. 21)

Ecology: Collected on common cow parsnip (Heracleum maximum). Collected from "sand dunes" and "outlet marsh" habitats. Collected April to September, although much more frequently collected from June through August. Collected from elevations of $320 m-3002 m$.

Discussion: The concept of limatus has been applied consistently since Hine erected the name in 1922. 
Type Material: "Savonoski/ Naknek Lake/ Alaska/.July '19", "Jas. S. Hine/ Collector", red label "Allotype", yellow label "PARALECTOTYPE/ Syrphus/ limatus/ Hine", barcode label "OSUC 0373263"; male; deposited in the Ohio State University Collection (OSU). (examined)

Material Examined:

Paralectotype: United States of America: Alaska : Savonoski; Naknek Lake, [58.497288 N, -155.266794 W], vii.1919, 1 female, OSUC 319689, Jas. S. Hine (OSU); Savonoski, Naknek Lake, [58.717222 N, -156.863889 W], vii.1919, 1 male, CNCD 87874, J. S. Hine (CNC); Other Material Examined: Canada: Alberta: Sulphur Mountain, Banff National Park, [51.149438 N, -115.583484 W], 2195m, 28.vii.1967, 1 female, CNCD 13699, J.R. Vockeroth (CNC); Banff, Banff National Park, [51.180275 N, -115.568433 W], 9.vi.1922, 1 male, CNCD 13647, C.B.D. Garrett (CNC); Mile 14 Banff, Banff-Jasper Highway, [51.231247 N, -115.838509 W], 3.viii.1955, 1 female, CNCD 13686, R. Coyles (CNC); Snow Creek Pass, Banff National Park, [51.60524 N, 115.808617 W], 2256m, 28.vii.1962, 1 female, CNCD 13687, W.R.M. Mason (CNC); British Columbia: Vaseux L. Hills to E, [49.316685 N, -119.523082 W], 2.v.1983, 1 male, JSS 20038, S.G. Cannings (UBCZ); Robson, [49.333015 N, -117.692871 W], 6.v.1958, 1 male, Royal British Columbia Museum ENT 99188122, H.R. Foxlee ( RBCM ); Robson, [49.333015 N, -117.692871 W], 11.v.1966, 1 male, Royal British Columbia Museum ENT 99188124, H.R. Foxlee ( RBCM ); Robson, [49.333015 N, 117.692871 W], 12.v.1956, 1 male, !SS 20040, H.R. Foxlee (UBCZ); Robson, [49.333015 N, -117.692871 W], 17.vii.1955, 1 female, JSS 20041, H.R. Foxlee 
(UBCZ); Robson, [49.333015 N, -117.692871 W], 28.iv.1958, 1 female, JSS 20043, H.R. Foxlee (UBCZ); Robson, [49.333015 N, -117.692871 W], 12.ix.1952, 1 female, ISS 20044, H.R. Foxlee (UBCZ); Robson, [49.333015 N, -117.692871 W], 13.v.1967, 1 male, JSS 20088, H.R. Foxlee (UBCZ); Robson, [49.333015 N, -117.692871 W], 22.v.1956, 1 male, JSS 20108, H.R. Foxlee (UBCZ); Robson, [49.333015 N, 117.692871 W], 27.vii.1959, 1 male, JSS 20129, H.R. Foxlee (UBCZ); Robson, Central Kootenay L.D., [49.342029 N, -117.697831 W], 15.v.1948, 1 male, CNCD 13656, H.R. Foxlee (CNC); Robson, Central Kootenay L.D., [49.342029 N, -117.697831 W], 11.v.1948, 1 male, CNCD 13657, H.R. Foxlee (CNC); Robson, Central Kootenay L.D., [49.342029 N, -117.697831 W], 11.v.1948, 1 male, CNCD 14473, H.R. Foxlee (CNC); Squamish, Diamond Head Trail, [49.811126 N, -123.07434 W], 975m, 9.viii.1953, 1 female; CNCD 13683, G.J. Spencer (CNC); M[oun]t Revelstoke, [50.98333333 N, 118.2 W], 1.viii.1952, 1 female, CNCD 533, G.P. Holland (CNC); Mount Harry, Mount Revelstoke National Park, [51.035979 N, -118.142509 W], 1890m, 20.vii.1952, 1 female, CNCD 13682, G.J. Spencer (CNC); Mt. Revelstoke, [51.046972 N, 118.139408 W], 13.viii.1952, 1 female, CNCD 48545, G. J. Spencer (CNC); Moosehorn Lake, Cassiar L.D., [58.164009 N, -132.12694 W], 1372m, 1.viii.1960, 1 female, CNCD 13678, W.W. Moss (CNC); Moosehorn Lake, Cassiar L.D., [58.164009 N, -132.12694 W], 1372m, 30.vii.1960, 1 female, CNCD 13679, R. Pilfrey (CNC); Summit Lake, Mile 392 of Alaska Highway, Northern Rockies R.D., [58.648201 N, 124.666912 W], 1280m, 21.vii.1959, 1 female, CNCD 13680, R.E. Leech (CNC); Summit Lake, Mile 392 of Alaska Highway, Northern Rockies R.D., [58.648201 N, 124.666912 W], 1433m, 15.vii.1959, 1 female, CNCD 13681, R.E. Leech (CNC); Liard 
R. hotsprings, [59.425991 N, -126.096398 W], 23.vii.1987, 1 female, JSS 20046, S.G. Cannings (UBCZ); Atlin, [59.5775 N, -133.69236 W], viii., 1 female, CNCD 13684, (CNC); Campbell Mtn., Pentiction, 880m, 13.v.1983, 1 male, JSS 20039, S.G. Cannings (UBCZ); Northwest Territories: Muskox Lake, [64.633135 N, -108.249285 W], 20.vii.1953, 1 male, CNCD 13645, J.G. Chillcott (CNC); 21 miles east Tuktoyaktuk, [69.426844 N, -132.172475 W], 2-5.vii.1971, 1 male, CNCD 13646, D.M. Wood (CNC); 21 miles east Tuktoyaktuk, [69.426844 N, -132.172475 W], 20-25.vi.1971, 1 female, CNCD 13694, D.M. Wood (CNC); Nova Scotia : Cape Breton Highlands National Park, Mackenzie Fire Tower, [46.773977 N, -60.818772 W], 8.vii.1983, 1 male, CNCD 13665, D.M. Wood (CNC); Quebec: Cté Missisquoi summit Mt Pinacle, [45.018184 N, -72.855064 W], 700m, 20.vi.1991, 1 male, JSS 20795, D.M. Wood (CNC); Knowlton, Brome-Missisquoi, [45.216716 N, -72.514769 W], 12.vi.1928, 1 female, CNCD 13651, W.J. Brown (CNC); Summit of Mt. Rigaud, [45.46638889 N, 74.32638889 W], 4.vi.1978, 4 males, CNCD 5809, 13659-13661, D.M. Wood (CNC); Mont Saint-Hilaire, La Vallee-du-Richelieu, [45.552948 N, -73.155276 W], 17.vi.1965, 1 male, CNCD 13667, J.W. Boyes (CNC); Mont Saint-Hilaire, La Vallee-duRichelieu, [45.552948 N, -73.155276 W], 27.vi.1965, 4 males, CNCD 13668-13671, J.W. Boyes (CNC); Laniel, Temiscamingue, [47.045828 N, -79.268979 W], 10.vi.1931, 1 female, CNCD 13688, H.S. Fleming (CNC); Sakami (LG3), [53.71666667 N, 76.06666667 W], 13-18.vii.1977, 2 males, CNCD 5808, 13658, D.M. Wood (CNC); Lac Duplanter, Lac Delorme, [54.883534 N, -69.78209 W], 7-12.vii.1977, 3 males, CNCD 13662-13664, D.M. Wood (CNC); Kuujjuaq (Fort Chimo), [58.100076 N, 68.406179 W], 28.vii.1948, 2 females, CNCD 13689, 13690, R.H. MacLeod (CNC); 
Yukon Territory: Carcross, sand dunes, [60.186691 N, -134.692703 W], 1618.vi.1982, 1 male, CNCD 13666, G. \& M. Wood (CNC); Whitehorse, [60.733402 N, 135.082092 W], 22.vii.1959, 1 female, CNCD 13698, R. Madge (CNC); Haines Jct., [60.752472 N, -137.510006 W], 29.vi.1974, 1 female, JSS 20181, G.G.E. Scudder (UBCZ); Otter Lake, [62.486817 N, -130.4166667 W], 1219m, 28.vii.1960, 1 female, CNCD 13650, E.W. Rockburne (CNC); Otter Lake, [62.486817 N, -130.4166667 W], 1219m, 29.vii.1960, 1 female, CNCD 13696, E.W. Rockburne (CNC); Sheldon Lake, [62.616763 N, -131.266603 W], 1067m, 3.vii.1960, 1 male, CNCD 13654, E.W. Rockburne (CNC); Sheldon Lake, [62.616763 N, -131.266603 W], 1067m, 3.vii.1960, 1 female, CNCD 13915, J.E.H. Martin (CNC); Sheldon Lake, [62.616763 N, 131.266603 W], 1067m, 7.vii.1960, 1 male, CNCD 13655, 1 female, CNCD 13695, J.E.H. Martin (CNC); La Force Lake, [62.683333 N, -132.333333 W], 1006m, 27.vi.1960, 1 male, CNCD 14010, J.E.H. Martin (CNC); Borth Fork Pass, [64.51666667 N, -138.2166667 W], 1067m, 27.vii.1982, 1 female, JSS 20045, S.G. Cannings, L. Vasington, R.A. Moore (UBCZ); North Fork Crossing, Mile 43 Peel Pit Road, [64.565983 N, -138.250648 W], 1067m, 6.vii.1962, 1 male, CNCD 13652, R.E. Leech (CNC); North Fork Crossing, Mile 43 Peel Pit Road, [64.565983 N, 138.250648 W], 1067m, 3.vii.1962, 1 male, CNCD 13653, P.J. Skitsko (CNC); North Fork Crossing, Ogilvie Mountains, [64.565983 N, -138.250648 W], 7.vii.1962, 1 female, CNCD 13697, P.J. Skitsko (CNC); km 82, Dempster Highway, [64.581259 N, 138.2617.21 W], 1300m, 20.vii.1981 21.vii.1981, 1 female, CNCD 13685, Lafontaine and G. \& M. Wood (CNC); Mile 87, Dempster Highway, [65.054721 N, -138.128324 W], 27-30.vi.1973, 1 male, CNCD 13649, G.\&D.M. Wood (CNC); Mile 87, Dempster 
Highway, [65.054721 N, -138.128324 W], 5-7.vii.1973, 1 female, CNCD 13691, G. \& D.M. Wood (CNC); Mile 87, Dempster Highway, [65.054721 N, -138.128324 W], 48.viii.1973, 1 female, CNCD 13692, G. \& D.M. Wood (CNC); Mile 87, Dempster Highway, [65.054721 N, -138.128324 W], 16-17.vii.1973, 1 female, CNCD 13693, G. \& D.M. Wood (CNC); Richardson M[oun]t[ain]s, [66.04166667 N, -135.5333333 W], 1219m, 6.vii.1982, 1 male, CNCD 5810, D.M. Wood (CNC); Richardson M[oun]t[ain]s, [66.525 N, $-135.1166667 \mathrm{~W}$ ], 762m, 7.vii.1982, 1 male, CNCD 532, M. Wood (CNC); km 416 Dempster Highway, [66.644581 N, -136.324471 W], 750m, 22-28.vi.1980, 3 males, CNCD 13675-13677, Wood \& Lafontaine (CNC); km 465, Dempster Highway, [67.046392 N, -136.209668 W], 800m, 5-7.vii.1980, 1 male, CNCD 13674, Wood \& Lafontaine (CNC); Richardson Mountains, [68.163651 N, 136.988868 W], 762m, 7.vii.1982, 2 males, CNCD 13672, 13673, D.M. Wood (CNC); British Mts., [69.21666667 N, -140.0833333 W], 320m, 21.vi.1984 26.vi.1984, 1 female, JSS 20774, G. \& M. Wood \& D. Lafontaine (CNC); United States of America: Alaska: Katmai, [58.033889 N, -154.876944 W], viii.1917, 1 female, CNCD 112302, J. S. Hine (CNC); Savonoski, Naknek River, Bristol Bay County, [58.716814 N, 156.865366 W], vii.1919, 1 male, JSS 19479, 1 female, JSS 19478, J.S. Hine (CNC); Matanuska, Matanuska-Susitna County, [61.541945 N, -149.229674 W], vii.1944, 3 females, JSS 19483, 19484, 19489, J. Chamberlin (CNC); Matanuska, [61.541945 N, 149.229674 W], vii.1944, 1 female, OSAC 133385, J. Chamberlin (OSAC); Healy, Denali County, [63.845424 N, -149.013681 W], 26.vi.1921, 1 female, JSS 19485, J.M. Aldrich (CNC); Healy, Denali County, [63.845424 N, -149.013681 W], 27.vi.1921, 1 male, JSS 19524, J.M. Aldrich (CNC); Unalakleet, [63.86666667 N, -160.7833333 W], 
15.viii.1961, 1 female, CNCD 26574, B.S. Heming (CNC); Unalakleet, [63.873056 N, 160.788056 W], 8.viii.1961, 1 female, CNCD 112193, B. S. Heming (CNC); Unalakleet, [63.873056 N, -160.788056 W], 9.viii.1961, 1 female, CNCD 112194, B. S. Heming (CNC); Umiat, [69.36666667 N, -152.1333333 W], 6.vii.1959, 1 female, CNCD 26579, J.E.H. Martin (CNC); Colorado: Tennessee Pass, Lake County, [39.357246 N, -106.313889 W], 24.vii.1917, 1 female, JSS 19490, J.M. Aldrich (CNC); Loveland Pass, Western Slope, [39.65 N, -105.8666667 W], 3002m, 28.vii.1961, 2 males, CNCD 26385, 26386, J.G. Chillcott (CNC); Doolittle Ranch, Mt. Evans, Clear Creek County, [39.675739 N, -105.601348 W], 2987m, 23.vii.1961, 1 male, CNCD 26383, B.H. Poole (CNC); Doolittle Ranch, Mt. Evans, Clear Creek County, [39.675739 N, -105.601348 W], 2987m, 22.vii.1961, 1 male, CNCD 26384, W.R.M. Mason (CNC); Doolittle Ranch, Mt. Evans, [39.675739 N, -105.601348 W], 8.vii.1961, 1 female, CNCD 111622, W. R. M. Mason (CNC); Doolittle Ranch, Mt. Evans, [39.675739 N, -105.601348 W], 11.viii.1961, 1 female, CNCD 112192, W. R. M. Mason (CNC); Doolittle Ranch, Mount Evans, [39.675739 N, -105.601348 W], 2987m, 23.vii.1961, 1 female, CNCD 35315, J.G. Chillcott (CNC); Chambers Lake, [40.603663 N, -105.853645 W], 3.ix.1932, 1 male, JSS 20739, (CSUC); North Park, [40.73 N, -106.18 W], 1.vii.1932, 1 female, JSS 20733, (CSUC); Glendevey, [40.8078 N, -105.935501 W], 7.vii.1931, 1 female, JSS 20741, C.R. Jones (CSUC); Maine: Mount Katahdin, Piscataquis County, [45.904968 N, -68.921017 W], 5.vii.1958, 1 male, JSS 19481, (CNC); Massachusetts: Wellesley Hills, Norfolk County, [42.308427 N, -71.278693 W], 1963, 1 female, ISS 19482, D. Meissner (CNC); Amherst, Hampshire County, [42.34038 N, -72.496818 W], 15.v.1963, 1 female, JSS 
19486, S.T. William (CNC); Amherst, Hampshire County, [42.34038 N, -72.496818 W], 8.v.1965, 1 female, JSS 19487, Mansfield (CNC); Amherst, Hampshire County, [42.34038 N, -72.496818 W], 8.v.1965, 1 male, JSS 19488, B. Scott (CNC); Amherst, Massachusetts, [42.34038 N, -72.496819 W], 6.v.1973, 1 male, USNM ENT 258065, A. Lavallee (USNM); New York: Mount MacIntyre Summit, Essex County, [44.132434 N, -74.007381 W], 26.vi.1941, 1 male, CNCD 26387, H. Dietrich (CNC); Mount Hurricane, Essex County, [44.233601 N, -73.708728 W], vi.1939, 1 male, JSS 19480, R.C. Shannon (CNC); Utah: Cowley Canyon, Cache County, [41.772669 N, 111.60407 W], 10-17.viii.1989, JSS 25132, W.J. Hanson (EMUS); Cache Co., Logan Cyn., Turner Campground, [41.85 N, -111.5666667 W], 2.vii.2003 6.vii.2003, 1 female, JSS 24607, W.J. Hanson \& Brammer (EMUS); Tony Grove Creek, Cache County, [41.886599 N, -111.564102 W], 8-15.vii.1994, JSS 24608, 25121-25124, 25127, W.J. Hanson (EMUS); Tony Grove Creek, Cache County, [41.886599 N, 111.564102 W], 12-19.viii.1994, JSS 24609, 25125, 25126, 25129, 25130, W.J. Hanson (EMUS); Tony Grove Creek, Cache County, [41.886599 N, -111.564102 W], 15-26.vii.1994, JSS 25128, W.J. Hanson (EMUS); Tony Grove Creek, Cache County, [41.886599 N, -111.564102 W], 4-12.viii.1994, JSS 25131, W.J. Hanson (EMUS); Cache Co., Tony Grove Crk, [41.892197 N, -111.634869 W], 29.vi.-8.vii.1994, 1 male, JSS 24610, W.J. Hanson (EMUS); West Hodges Canyon, Cache County, [41.910305 N, -111.42277 W], 8.viii.1978 11.viii.1978, JSS 25133, Hanson, Knowlton (EMUS).

\section{Dasysyrphus lotus (Williston)}


Figures: 9A, C, 14A, 22 .

Synonyms and References:

Syrphus lotus Williston, 1887: 75 (original description)

Metasyrphus lotus

Fluke, 1933: 108 (key, redescription and transfer to Dasysyrphus)

Dasysyrphus lotus

Stone et al., 1965: 564 (catalogue and transfer to Dasysyrphus)

Vockeroth, 1992: 67 (key and redescription)

Diagnosis: Abdominal maculae do not cross abdominal margin; maculae on tergites $3 \& 4$ oblique and usually meet in the middle; face and gena continuously light (Figs $9 A, C)$.

Description:

Body Length: $9.4-11.7 \mathrm{~mm}$; Wing Length: $8.7-11.4 \mathrm{~mm}$

Head: Frons dark with light pollenose fascia (in males it runs along ventral edge of where eyes meet, in females it is complete and $>1 / 2$. the length between the antennae and ocelli) and dark pile; face light with dark vitta $1 / 4-1 / 3$ the width of the face and does not reach the antennal socket, face with light pile lateral to the eye, sometimes dark pile along fascia or dorsally, near frons; gena light (Fig. 9C) with light pile; 
occiput dark, covered in dense, light pollen, pile light, some with some dark; scape, pedicel and flagellomere dark.

Thorax: Scutum dark, sometime shiny or metallic, pile light; scutellum light with light pile along anterior edge, dark posteriorly; wing cell $c$ and $r_{1}$ bare at base, br bare above spurious vein and small to large areas below, bm bare on most of anterior half, and cup bare under CuP; haltere light; femora basally dark, apically light, tibiae and tarsi light, metatarsus slightly darkened anteriorly.

Abdomen: Maculae on tergite 2 large and triangular (lateral edge flattened and medial edge with rounded point), $1 / 3-<1 / 2$ width of tergite, maculae on tergites $3 \& 4$ are oblique and fairly straight, usually meeting medially, fairly uniform in thickness, narrowing toward medial edge slightly, maculae do not reach abdominal margin (Figs 9A, C); sternites light with dark fasciae, anterior edge of fascia on sternite two usually angled forming a point in the centre.

Male Genitalia: Surstylus more or less triangular in shape in lateral view, with posterodorsal edge rounded, long pile on posteriolateral surface, spines on anteroventral edge; cercus oval with long pile over entire surface; gonostylus hookshaped with few pile on dorsal surface; basiphallus elongate, basally bent at about a 90 degree angle towards dorsum, apical end curved ventrally and fully sclerotized all the way around without pile or spines; distiphallus elongate, apical end flared out into small horn, which is mostly sclerotized but membranous at apex with 
minute setulae dorsally, basally curved towards dorsum and not enlarged (Fig. $14 A)$.

Intraspecific variation: Abdominal maculae on tergites $3 \& 4$ usually meet medially, but sometimes they are completely separated from each other.

Etymology: The word lotus is Latin, meaning washed.

Distribution: Southwestern USA (AZ, CA) and Mexico (Fig. 22).

Ecology: Collected from March to November at elevations from 1524-2743m.

Discussion: Williston (1887) discusses two different specimens in his species description. The first, the type, and another that he speculates is "allied". This second specimen is reported to have several differences, mainly the shape of the abdominal maculae and gena colour. Williston (1887) describes this second specimen as having a "black stripe on the cheeks" and he describes the abdominal maculae as being "more oblique, and strongly concave in front" and "more convex behind". In 1895 Snow describes this lotus variation as D. creper. Fluke (1933) states that most published records of lotus probably refer to creper and that most of the specimens identified as lotus agree with Williston's (1887) variation (the female from New Mexico). Fluke postulates that the specimen might be teneral due to the light head, but then goes on to say that it does not explain the differently shaped 
maculae. Curran's (1925) redescription of lotus also refers to creper. It is clear from the description of the abdominal maculae and the figure that he was not looking at Williston's (1887) lotus.

Dasysyrphus lotus has a high mean intraspecific variation of 3.09\% (Table 2). In the NJ tree (Fig. 4), the two Nearctic specimens cluster closely together $(1.34 \%$ different), while the Neotropical specimen is $3.29-4.38 \%$ different from the others. This is highly suggestive that lotus is a complex as currently defined. With an extremely similar habitus among these three specimens as well as the 21 specimens where it was not possible to extract DNA, it can be concluded that lotus s.l. occurs from the southwestern Nearctic through to the Neotropical region, but that it likely includes at least two species. Further investigation needs to be conducted to help discern whether the Neotropical specimens are indeed a new species.

Type Material: “Arizona,/ C.U. Lot 35”, “Acc. 19,702/ Williston”, "Syrphus/ lotus/ Will.", red label "Type/ No. 129/ U.S.N.M.", "Jeff Skevington/ Specimen \#/ 23780"; female; deposited in the United States National Museum of Natural History, Washington, D.C (USNM). (examined)

Material Examined:

Mexico: 10 Miles West of El Salto, [23.727593 N, -105.49364 W], 2743m, 10.vii.1964, 2 males, CNCD 26391, 26392, J.F. McAlpine (CNC); Amecameca, [19.119148 N, -98.764597 W], ix.1900, 1 male, USNM ENT 35111, 0.W. Barrett 
(CNC); Atlacomulco, 8.x.1954, 1 male, JSS 21093, R. Dreisbach (MSUC); United States of America: Arizona: Ramsey Canyon, 15 Miles South of Sierra Vista, Huachuca Mountains, [31.337025 N, -110.302646 W], 1585m, 28.vii.1967, CNCD 60065, R.F. Sternitzky (CNC); Carr Canyon, 15 miles South of Sierra Vista, Cochise County, [31.440158 N, -110.289641 W], 1524m, 29.x.1967, 1 male, CNCD 26388, 2 females, CNCD 26393, 26394, R.F. Sternitzky (CNC); Huachuca Mountains, Ramsey Canyon, 1.4 miles along Hamburg trail, [31.446077 N, $-110.311099 \mathrm{~W}], 1920 \mathrm{~m}$, 23.ix.1994, 1 male, JSS 18303, J.E. O'Hara (CNC); Ramsey Canyon, 15 Miles South of Sierra Vista, Cochise County, [31.447697 N, -110.307712 W], 1585m, 1.ix.1967 15.ix.1967, 1 male, CNCD 26389, R.F. Sternitzky (CNC); Ramsey Canyon, 15 Miles South of Sierra Vista, Cochise County, [31.447697 N, -110.307712 W], 1585m, 12.vi.1967, 2 females, CNCD 26395, 26396, R.F. Sternitzky (CNC); Ramsey Canyon, 15 Miles South of Sierra Vista, Cochise County, [31:447697 N, -110.307712 W], 1585m, 10.iv.1967, 1 female, CNCD 26397, R.F. Sternitzky (CNC); Ramsey Canyon, 15 Miles South of Sierra Vista, Cochise County, [31.447697 N, -110.307712 W], 1585m, 1-8.x.1967, 1 female, CNCD 26398, R.F. Sternitzky (CNC); Canelo, Santa Cruz County, [31.542591 N, -110.514478 W], 1372m, 17.iii.1967, 1 male, CNCD 26390, R.F. Sternitzky (CNC); Mount Lemmon, San Catalina Mountains, Pima County, [32.443131 N, -110.788429 W], 2377m, 29.vii.1917, 1 female, CNCD 26399, (CNC); Huachuca Mts.; Ramsey Cyn.; 15 mi. S. Sierra Vista, 15.xi.1967, 1 male, CNCD 106795, R.F. Sternitzky (CNC); Huachuca Mts.; Miller Can., 3.v.1948, 1 male, JSS 23760, A.L. Melander (USNM); California: Blodgett Experimental Forest, Eldorado Co., [38.87882 N, -120.659071 W], 7.vi.1975, 1 male, JSS 18302, D. Voeatlia (CNC). 


\section{Dasysyrphus nigricornis (Verrall)}

Figures: 7A-C, $14 \mathrm{~B} \& 22$

Synonyms and References:

Scaeva obscura Zetterstedt, 1838: 601 (original description, name preoccupied)

Bicik \& Laska, 1996 (lectotype designation and discussion)

Syrphus nigricornis Verrall, 1873, 251 (new name given, short description and transfer to Syrphus)

Verrall, 1901: 353 (discussion)

Becker, 1921: 44 (redescription)

Syrphus obscuratus Ringdahl, 1928: 19 (original description)

Syrphus nigricornis var. obscuraticeps Frey, 1950: 10 (original description)

Dasysyrphus nigricornis Verrall:

Peck, 1974: 128 (key and transfer to Dasysyrphus)

Peck, 1988: 15 (catalogue)

Barkalov, 2007: 283 (key and redescription)

Speight, 2011: 70 (discussion)

Diagnosis: Abdominal maculae of tergites $3 \& 4$ oblique, fairly uniform in thickness with slight constriction in the middle and do not cross abdominal margin (Figs 7AC). Maculae on tergite 2 of male small ( $<1 / 4$ width of tergite), triangular to oval and 
close to lateral edges (Fig. 7A), in females are larger ( $1 / 4-1 / 3$ width of tergite) and narrowly oval (Fig. 7C); usually darker in appearance due to reduced maculae. Wing densely microtrichose. Similar in appearance to D. pauxillus (Fig. 11A), pacificus (Fig. 10D) and laticaudus (Fig. 7E).

Description:

Body Length: $5.5-7.7 \mathrm{~mm}$; Wing Length: $4.5-7.7 \mathrm{~mm}$

Head: Frons dark with light pollenose fascia (in males it runs along ventral edge of where eyes meet, in females it is complete and $1 / 3-1 / 2$ the length between the antennae and ocelli) and dark pile; face light with dark vitta $1 / 4-1 / 2$ the width of the face, usually not reaching antennal socket, face with dark pile lateral to the eye; gena typically dark pile, but sometimes light; occiput dark, covered in light pollen, pile light; scape and pedicel dark, flagellomere dark.

Thorax: Scutum dark, sometime shiny or metallic, pile varies between all light and dark anteriorly and light posteriorly; scutellum light to dark with darker lateral edges, light pile sometimes with light and dark pile; wings entirely microtrichose with no bares areas in any cell; haltere light; femora dark with light apical end, tibiae light basally and dark apically, dark. 
Abdomen: Maculae on tergite 2 small ( $<1 / 4$ width of tergite), triangular to oval and close to lateral edges in males (Fig. 7A), larger $(1 / 4-1 / 3$ width of tergite) and narrowly oval in females (Fig. 7C), maculae on tergites $3 \& 4$ are oblique, fairly uniform in width with slight constriction in the middle, maculae do not reach abdominal margin (Figs 7A-C); sternites light with dark fasciae varying in thickness.

Male Genitalia: Surstylus more or less triangular in shape in lateral view, flattened posteriorly, long pile on dorsal, laterodorsal and posterodorsal sides, row of spines on posteriomedial edge; cercus oval with long pile over entire surface; gonostylus boot-shaped with projection on posterodorsal surface that projects posterodorsally, pile on dorsal surface; basiphallus elongate, basally bent at 135 degree angle towards anterodorsum, apical end curved ventrally and is partly membranous dorsally, with spines on posterodorsal side; distiphallus elongate, apical end flared out into horn, which is mostly sclerotized but membranous at apex with minute setulae dorsally, basally bent towards dorsum and enlarged ventrally at bend (Fig. 14B).

Intraspecific variation: Maculae on tergite two can vary in width. In males maculae are typically $<1 / 4$ the width of the tergite, but in rare occasions may be up to $1 / 3$ of the width. In females hey are typically $1 / 4-1 / 3$ the width. Maculae on tergite $3 \& 4$ in the male look similar to those of pauxillus, pacificus and laticaudus, however in the female the maculae tend to be slightly thinner than similar species and sometimes are more narrow on the inner edge. 
Etymology: From the Latin niger, meaning black, dark, dusky, and cornus, meaning horn (possibly referring to antennae).

Distribution: Northern Canada (MB, NL, NT, QC, YT), Northern USA (AK) and Greenland. (Fig. 22)

Ecology: Has been collected from late May - July, from elevations of 762-1250m.

Discussion: Dasysyrphus nigricornis is recognized from the Nearctic for the first time. Long buried within pauxillus in this region, specimens from northern Canada and the US are morphologically and genetically different from pauxillus and the two other species also split off from it here, laticaudus and pacificus. Male genitalia of these Nearctic specimens match the genitalia of the nigricornis paralectotype figured by Bicik \& Laska (1996, Fig. A). Part of the reason that this species has not been recognized in this region before is that much confusion has existed over the use of the name in the Palaearctic. When Verrall provided a new name for obscura in 1873 , because the name was preoccupied, he used specimens from Scotland for his description. However, obscura was described by Zetterstedt from Scandinavia and is not known to occur in the UK (Bicik \& Laska 1996; Speight 2011). The material used by Verrall in his redescription is apparently actually pinastri, leading to the obvious confusion. In 1996, Bicik \& Laska examined the type series of obscura to help straighten out the nomenclature. Upon their examination of the type series 
they found three different species, three specimens of nigricornis s.s., two of pauxillus s.l. and one of pinastri. They nominated a lectotype to stabilize the name, as well as provided figures of genitalia and characters to differentiate nigricornis from pauxillus s.l. and pinastri.

Despite being morphologically distinct from $D$. occidualis, $D$. nigricornis cannot be separated from occidualis by COI DNA.

Type Material: "S. obscura Juckasj." (Zetterstedt label), "S. obscuraticeps Frey" (Heese label, 1969), number: "185", male, deposited in the Museum of Zoology, Lund University (MZLU), Lund, Sweden (Bicik \& Laska 1996). (not examined)

Material Examined:

Canada: Manitoba: Warkworth Creek, Near Churchill, [58.507672 N, -93.997769 W], 10.vi.1952, 1 male, CNCD 13766, C.D. Bird (CNC); Churchill Area, Burn site $\mathrm{N}$ [orth] of Twin lakes, [58.61861111 N, -93.82888889 W], 29.vi.2007, 3 males, CNCD 9204-9206, J. Skevington (CNC); Churchill Area, Bluff D at Bird Cove, [58.76166667 N, -93.89833333 W], 30.vi.2007, CNCD 9274, J. Skevington (CNC); Newfoundland and Labrador: Pinware River Provincial Park, Division No. 9, [51.634267 N, -56.708114 W], 29.vi.1983, 1 male, CNCD 13902, A. Borkent (CNC); Cartwright, Labrador, [53.694772 N, -57.010406 W], 29.vi.1955, 1 female, CNCD 13903, E.E. Sterns (CNC); Northwest Territories: Resdelta, Great Slave Lake, [66.051734 N, -120.780449 W], 22.vi.1944, 1 female, CNCD 13777, J.R. Vockeroth 
(CNC); Km 491 Dempster Highway, [67.182795 N, -135.791046 W], 26.vi.1982, 1 male, CNCD 13772, G. \& M. Wood (CNC); Aklavik, [68.219638 N, -135.010707 W], 30.vi.1956, 1 female, CNCD 13776, E.F. Cashman (CNC); Reindeer Depot, Mackenzie Delta, [68.668974 N, -134.071574 W], 25.vi.1948, 1 male, CNCD 13712, J.R.

Vockeroth (CNC); Reindeer Depot, Mackenzie Delta, [68.668974 N, -134.071574 W], 27.vi.1948, 2 males, CNCD 13714, 15384, J.R. Vockeroth (CNC); Reindeer Depot, Mackenzie Delta, [68.668974 N, -134.071574 W], 28.vi.1948, 1 male, CNCD 13713, J.R. Vockeroth (CNC); Reindeer Depot, Mackenzie Delta, [68.668974 N, -134.071574 W], 6.vii.1948, 1 male, CNCD 13715, J.R. Vockeroth (CNC); Granet Lake, Franklin, [68.701471 N, -125.58813 W], 20.vii.1969, 1 female, CNCD 13778, G.E. Shewell (CNC); 21 miles east Tuktoyaktuk, [69.426844 N, -132.172475 W], 20-25.vi.1971, 4 males, CNCD 13702-13704, 13707, D.M. Wood (CNC); 21 miles east Tuktoyaktuk, [69.426844 N, -132.172475 W], 2-5.vii.1971, 1 male, CNCD 13705, 1 female, CNCD 13774, D.M. Wood (CNC); 21 miles east Tuktoyaktuk, [69.426844 N, -132.172475 W], 8-12.vii.1971, 1 female, CNCD 13775, D.M. Wood (CNC); 20 miles East of Tuktoyaktuk, [69.427058 N, -132.212732 W], 21.vi.1971, 1 male, CNCD 13706, W.R.M. Mason (CNC); Tuktoyaktuk, [69.438369 N, -133.01637 W], 17.vi.1971, 3 males, CNCD 13709-13711, D.M. Wood (CNC); Quebec: Kuujjuaq (Fort Chimo), [58.100076 N, -68.406179 W], 23.vii.1948, 1 female, CNCD 13879, R.H. MacLeod (CNC); Yukon Territory: Swim Lakes, [62.210135 N, -132.809978 W], 975m, 11.vi.1960, 1 female, CNCD 13568, J.E.H. Martin (CNC); Swim Lakes, [62.210135 N, 132.809978 W], 975m, 15.vi.1960, 1 female, CNCD 13733, J.E.H. Martin (CNC); Swim Lakes, [62.210135 N, -132.809978 W], 975m, 25.vi.1960, 2 females, CNCD 
13734, 13735, J.E.H. Martin (CNC); Sheldon Lake, [62.616763 N, -131.266603 W], 1067m, 7.vii.1960, 2 females, CNCD 13737, 13738, E.W. Rockburne (CNC); La Force Lake, [62.683333 N, -132.333333 W], 1006m, 7.vii.1960, 1 female, CNCD 13722, J.E.H. Martin (CNC); La Force Lake, [62.683333 N, -132.333333 W], 1006m, 9.vii.1960, 1 female, CNCD 13723, J.E.H. Martin (CNC); La Force Lake, [62.683333 N, -132.333333 W], 10.vii.1960, 2 females, CNCD 13724, 13725, J.E.H. Martin (CNC); La Force Lake, [62.683333 N, -132.333333 W], 1006m, 26.vi.1960, 1 female, CNCD 13726, J.E.H. Martin (CNC); La Force Lake, [62.683333 N, -132.333333 W], 1006m, 7.vii.1960, 1 female, CNCD 13727, E.W. Rockburne (CNC); La Force Lake, [62.683333 N, -132.333333 W], 1006m, 11.vii.1960, 1 female, CNCD 13729, E.W. Rockburne (CNC); La Force Lake, [62.683333 N, -132.333333 W], 13.vii.1960, 2 females, CNCD 13730, 13731, E.W. Rockburne (CNC); La Force Lake, [62.683333 N, 132.333333 W], 1006m, 25.vi.1960, 1 female, CNCD 13732, E.W. Rockburne (CNC); Hunter Creek, Dawson, [63.779 N, -138.61 W], 914m, 3.vii.1949, 1 male, CNCD 13701, 1 female, CNCD 13740, P.F. Bruggemann (CNC); Dawson, [64.050017 N, 139.41052 W], 16.vi.1949, 1 female, CNCD 13741, W.W. Judd (CNC); North Fork Pass, Ogilvie Mountains, [64.565983 N, -138.250648 W], 1250m, 12.vi.1962, 1 male, CNCD 13700, R.E. Leech (CNC); North Fork Crossing, Mile 43 Peel Pit Road, [64.565983 N, $-138.250648 \mathrm{~W}], 1067 \mathrm{~m}, 4 . v i i .1962,1$ female, CNCD 13736, P.J. Skitsko (CNC); Mile 51, Dempster Highway, [64.605992 N, -138.33789 W], 1721.vi.1973, 1 male, CNCD 13708, 1 female, CNCD 13739, G. \&D.M. Wood (CNC); km 465, Dempster H[igh]w[a]y, [67.046392 N, -136.209668 W], 25-26.vi.1982, 2 males, CNCD 5811, 13720, 1 male and 1 female (pinned together), CNCD 13719, G. \& M. 
Wood (CNC); Wright Pass Dempster Hwy., [67.083297 N, -136.162751 W],

9.vii.1985, 1 female, JSS 20169, S.G. Cannings (UBCZ); Richardson Mountains,

[68.163651 N, - $136.988868 \mathrm{~W}], 762 \mathrm{~m}, 12 . v i i .1982,1$ male and 1 female, CNCD 13721, M. Wood (CNC); Greenland: Sondrestrom Air Base; Near Sugar Loaf Mountain, [67.010556 N, -50.709167 W], 11.vii.1963, 1 male, JSS 23779, T.J. Curtin (USNM); United States of America: Alaska: Matanuska, Matanuska-Susitna County, [61.541945 N, -149.229674 W], 28.v.1945, 1 female, JSS 19518, J.C. Chamberlin (CNC); Matanuska, Matanuska-Susitna County, [61.541945 N, 149.229674 W], 7.vi.1944, 1 female, JSS 19519, J.C. Chamberlin (CNC); Matanuska, Matanuska-Susitna County, [61.541945 N, -149.22.9674 W], 7.vi.1944, 2 males, JSS 19520, 19521, J.C. Chamberlin (USNM); Mount Fairplay, Mile 32 Taylor Highway, [63.679038 N, -142.262944 W], 1097m, 10.vii.1962, 1 female, CNCD 26443, P.J. Skitsko (CNC); Nome Area, Mile 17 of Kougarok Road, [64.70611111 N, 165.2972222 W], 26.vi.2005 28.vi.2005, 1 female, CNCD 30896, J.\&R. Skevington (CNC); Umiat, [69.36666667 N, -152.1333333 W], 24.vi.1947, 1 male, JSS 19522, K.L. Knight (USNM).

Dasysyrphus occidualis Locke \& Skevington sp. nov.

Figures: 10A-C, 12B \& 23

Synonyms and References:

Syrphus amalopis Osten Sacken: 
Williston, 1887: 69 (in part; description)

Metasyrphus amalopis:

Fluke, 1933: 110 (in part; key and redescription)

Dasysyrphus amalopis:

Stone et al., 1965: 563 (in part; catalogue)

Vockeroth, 1992: 63 (in part; key and redescription)

Diagnosis: Abdominal maculae do not meet abdominal margin; maculae on tergites $3 \& 4$ are constricted medially and are either divided or not (Figs 10A-C). Wings cell br usually with a small bare area above base of spurious vein. Surstylus with a prominent posterolateral edge (Fig. 12B). Similar to D. amalopis, but D. amalopis has maculae on tergites $3 \& 4$ usually undivided (Fig. 5A), cell br densely microtrichose and does not have a prominent posterolateral edge on the surstylus (Fig. 12A). Male genitalia should be used to reliably differentiate between these two species. There is a range of overlap in southern Yukon where it may not be possible to distinguish between females of these two species due to variations in the aforementioned characters.

Description:

Body Length: 8.0 - 11mm; Wing Length: $7.2-10.7 \mathrm{~mm}$ 
Head: Frons dark with light pollenose fascia (in males it runs along ventral edge of where eyes meet, in females it is continuous, $1 / 3-1 / 2$ the length between the antennae and ocelli) and dark pile; face light with dark vitta $<1 / 4-1 / 3$ the width of the face, usually not reaching antennal socket, if it does then narrowly so, face with dark pile; gena dark with dark pile ventral to the eye and light pile elsewhere; occiput dark, covered in light pollen, pile light; scape, pedicel and flagellomere dark.

Thorax: Scutum dark, sometime shiny or metallic, pile dark and light, more light anteriorly and posteriorly; scutellum light to dark with darker lateral edges, some light pile along anterior edge and dark pile elsewhere; wing densely microtrichose, cell br usually with small bare areas at above base of spurious vein, $r_{1}$ sometimes bare at base and cup sometimes bare in areas along vein CuP; haltere light; femora basally dark, apically light, tibiae light basally, dark apically, tarsi dark.

Abdomen: Maculae on tergite 2 large, $1 / 3-<1 / 2$ the width of tergite 2 , and oval with constriction medially (sometimes just slight constriction), maculae on tergites $3 \& 4$ are oblique and constricted medially, usually completely dividing maculae into four spots, if not completely divided then maculae on tergite 4 usually more constricted that on tergite three and almost divided, maculae do not reach abdominal margin (Figs 10A-C); sternite 2 is light with a dark triangular to straight macula connecting with the lateral edges of the sternite, sternites $3 \& 4$ are light with dark fasciae of varying thickness. 
Male Genitalia: Surstylus more or less triangular in shape in lateral view, flattened posteriorly with prominent posterolateral edge, long pile on dorsal, laterodorsal and posterodorsal sides, row of spines on posteriomedial edge; cercus oval with long pile over entire surface; gonostylus boot-shaped with pointed projection on posterodorsal surface that projects dorsally, pile on dorsal surface; basiphallus elongate, basally bent at about a 135 degree angle towards the anterodorsum, apical end curved ventrally with a widened apex and is partly membranous dorsally, with spines on posterodorsal side, apex ; distiphallus elongate, apical end flared out into horn, which is mostly sclerotized but membranous at apex with minute setulae dorsally, basally bent towards dorsum and enlarged ventrally at bend (Fig. 12B).

Intraspecific variation: The maculae on tergites $3 \& 4$ are often divided (Fig. 10B). If not completely divided then maculae on tergite 4 usually more constricted that on tergite three and almost divided. Can have maculae on tergites $3 \& 4$ entire, but this less common (Fig. 10A). Wing densely microtrichose, except for cell br, which is usually bare above base of spurious vein, sometimes only in very tiny areas, with one or two microtrichia missing. Also, $\mathrm{r}_{1}$ sometimes bare at base and cup sometimes bare in areas along vein CuP.

Etymology: From the Latin occidualis, meaning western. 
Distribution: Western Canada (AB, BC, YT) and western USA (AK, CO, WA, WY) (Fig. 23).

Ecology: Collected from May - August, but more frequently collected in July. Collected at elevations of $613-3658 \mathrm{~m}$. Habitats listed on labels include "outlet marsh", "sedge fen" and "subalpine222".

Discussion: Until now, occidualis has been hidden within amalopis. With an absence of distinguishing external morphological characters, additional data were necessary to illuminate cryptic species such as this. In this study, COI DNA first highlighted the existence of two distinctly different genetic clusters within amalopis. Upon further inspection of the specimens, the male genitalia showed a prominent posterolateral edge on the surstylus (Fig. 12B), which amalopis does not have (Fig. 12A). When their geographic ranges were studied it was found that specimens of occidualis were only located in western Canada and the US (from Alaska to Colorado), while amalopis was found only in northern Canada. There is an overlap zone in southern Yukon so specimens in this area must be carefully studied to make the correct species determination.

Type Material: “ USA: WA: Mt. Baker-/ Snoqualmie NF: Mt. Baker:/ Silver Fir Campground; $2012 \mathrm{ft} . / 48.904950^{\circ} \mathrm{N}, 121.697917^{\circ} \mathrm{W}$; / 8.vii.2010; M.M.Locke", "J. Skevington/ Specimen \#/ 23708", "HOLOTYPE/ Dạsysyrphus/ occidualis Locke \& 
Skevington/ Det. M.M. Locke", male, deposited in the Canadian Collection if Insects, Arachnids and Nematodes (CNC), Ottawa, Ontario, Canada.

Material Examined:

Canada: Alberta: Kananaskis, Forest Experimental Station Seebe, [51.100879 N, 115.087692 W], 15.vi.1968, 1 female, CNCD 5014, H.J. Teskey (CNC); Banff, Banff National Park, [51.180275 N, -115.568433 W], 15.vii.1922, 3 males, CNCD 1362113623, C.B.D. Garrett (CNC); Banff, Banff National Park, [51.180275 N, -115.568433 W], 1 female, JSS 19432, N.B. Sanson (CNC); [54.962586 N, -114.799987 W], 11.vii.1951, 1 female, CNCD 13619, (CNC); British Columbia: Cathedral Prov. Park, Glacier Lake, [49.055785 N, -120.207299 W], 6.vii.1986, 1 female, Royal British Columbia Museum ENT 9882357, R.A. Cannings (RBCM); Cathedral Prov. Park, fen, Lk. of the Woods trail, [49.08 N, -120.18 W], 26.vii.1988, 1 female, JSS 20186, S.G. Cannings (UBCZ); Squamish, Diamond Head Trail, [49.811126 N, -123.07434 W], 1219m, 11.viii.1953, 1 female, CNCD 13610, W.R.M. Mason (CNC); Duffey L. Blowdown Cr., lake S. of Gott Peak, [50.40416667 N, -122.3416667 W], 21.vii.1988, 1 female, Royal British Columbia Museum ENT 9918197, C.S. Guppy (RBCM); Cornwall Hills Lookout, [50.7 N, -121.5 W], 2037m, 14.vi.1989, 1 male, Royal British Columbia Museum ENT 990626, R.A. Cannings \& C.S. Guppy (RBCM); Kootenay National Park, Daer-Pitts Aspen Control 2, [50.776662 N, -115.936917 W], 17.vi.2.vii.2000, 2 males, CNCD 30908, 30909, 1 female, CNCD 30903, G. Gareau (CNC); Mount Revelstoke National Park, [51.035979 N, -118.142509 W], 1828m, 26.vii.1952, 1 female, CNCD 13612, G.P. Holland (CNC); Mount Revelstoke National 
Park, [51.035979 N, -118.142509 W], 1768m, 15.viii.1952, 1 female, CNCD 13613, G.J. Spencer (CNC); Mount Harry, Mount Revelstoke National Park, [51.035979 N, 118.142509 W], 2073m, 8.viii.1952, 1 female, CNCD 13614, G.J. Spencer (CNC); Mt. Revelstoke, [51.05 N, -118.1333333 W], 1829m, 7.viii.1952, 1 female, Royal British Columbia Museum ENT 99188105, G.P. Holland (RBCM); Floe Lake, Kootenay National Park, [51.054 N, -116.137 W], 1981m, 9.viii.1955, 1 female, CNCD 13611, J.R.W. McGillis (CNC); Eva Lake Trail, Mount Revelstoke National Park, [51.079905 N, -118.108837 W], 1828m, 6.viii.1952, 1 female, CNCD 13615, G.J. Spencer (CNC); Tlell, Queen Charlotte Islands, Skenna-Queen Charlotte R.D., [53.562591 N, 131.93235 W], 25.v.1957, 1 male, CNCD 13620, E.E. MacDougall (CNC); Summit Lake, Mile 392 of Alaska Highway, Northern Rockies R.D., [58.648201 N, 124.666912 W], 1372m, 1.vii.1959, 1 female, CNCD 13603, R.E. Leech (CNC); Summit Lake, Mile 392 of Alaska Highway, Northern Rockies R.D., [58.648201 N, 124.666912 W], 1524m, 6.vii.1959, 1 female, CNCD 13604, E.E. MacDougall (CNC); Summit Lake, Mile 392 of Alaska Highway, Northern Rockies R.D., [58.648201 N, 124.666912 W], 1372m, 1.vii.1959, 1 female, CNCD 13605, E.E. MacDougall (CNC); Summit Lake, Mile 392 of Alaska Highway, Northern Rockies R.D., [58.648201 N, 124.666912 W], 1372m, 2-4.vii.1959, 3 females, CNCD 13606-13608, E.E.

MacDougall (CNC); Ft. Nelson, [58.804594 N, -122.697436 W], 4.vii.1949, 1 female, JSS 19993, J.K. Morie (UBCZ); Liard R. hotsprings, [59.425991 N, -126.096398 W], 23.vii.1987, 3 females, JSS 20176-20178, S.G. Cannings (UBCZ); Fireside, [59.666676 N, -127.14242 W], 24,v, 1980, CNCD 60083, Bruce Gill (CNC); 7 miles N Lions Bay, North of Vancouver, 18.v.1979, 1 male, CNCD 5463, G.F. Hevel (CNC); 
Spanish Hills, Galiano Island, 10.v.1980, CNCD 60094, G.G.E. Scudder (CNC); Yukon Territory: Carcross Dunes at Bennett L. beach, [60.17066 N, $-134.71809 \mathrm{~W}], 666 \mathrm{~m}$, 25.vi.2009, 1 female, JSS 20819, Lee Mennell (RBCM); Takhini R., west dunes $6.8 \mathrm{~km}$ NNE of Kusawa L. outlet, [60.66824 N, -136.07664 W], 19.vi.2009, 1 female, JSS 20820, S. Cannings (RBCM); Sheldon Lake, [62.616763 N, -131.266603 W], 914m, 21.vii.1960, 1 female, CNCD 13561, J.E.H. Martin (CNC); North Klondike River, Km 64 Dempster Highway, [64.45056 N, -138.218356 W], 9.vi.1980, 1 male, JSS 18376, R.J. Cannings (CNC); United States of America: Alaska: Matanuska, MatanuskaSusitna County, [61.541945 N, -149.229674 W], 29.v.1944, 1 female, JSS 19431, J. Chamberlin (CNC); Nome Area, Mile 17 of Kougarok Road, [64.70611111 N, 165.2972222 W], 26-28.vi.2005, 1 female, CNCD 30899, J.\&R. Skevington (CNC); Colorado: Pike's Peak Summit, [38.840342 N, -105.045006 W], 14.viii.1977, 1 female, USNM ENT 258091, A.B. Gurney (USNM); Ragged Mountain, Gunnison County, [39.118885 N, -107.407678 W], 20.vi.1950, 1 male, CNCD 26345, C.A. Downing (CNC); Mount Evans, Park County, [39.25 N, -106.1666667 W], 3658m, 3.viii.1961, 2 females, CNCD 26330, 26331, S.M. Clark (CNC); Mount Evans, Park County, [39.25 N, -106.1666667 W], 3658m, 3.viii.1961, 2 females, CNCD 26332, 26333, W.R.M. Mason (CNC); Mount Evans, Park County, [39.25 N, -106.1666667 W], 3444m, 10.viii.1961, 1 female, CNCD 26334, C.H. Mann (CNC); Mount Evans, Park County, [39.25 N, -106.1666667 W], 3353m, 22.vii.1961, 1 female, CNCD 26335, J.G. Chillcott (CNC); Mount Evans, Park County; [39.25 N, -106.1666667 W], 3658m, 4.viii.1961, 1 male, CNCD 26342, I.R. Stainer (CNC); Guanella Pass, Clear Creek County, [39.594992 N, -105.711109 W], 3536m, 14.vii.1961, 1 female, CNCD 
26340, S.M. Clark (CNC); Timberline, Mount Evans, Clear Creek County, [39.645575 N, -105.594897 W], 3566m, 22.vii.1961, 1 female, CNCD 26336, C.H. Mann (CNC); Timberline, Mount Evans, Clear Creek County, [39.645575 N, -105.594897 W], 3566m, 22.vii.1961, 1 female, CNCD 26337, B.H. Poole (CNC); Echo Lake, Mt. Evans, Clear Creek County, [39.658085 N, -105.6035 W], 3231m, 22.vii.1961, 1 female, CNCD 26338, J.G. Chillcott (CNC); Doolittle Ranch, Mt. Evans, Clear Creek County, [39.675739 N, -105.601348 W], 2987m, 12.viii.1961, 1 female, CNCD 26339, J.R. Stainer (CNC); Brainard Lake Rec. Area 4mi W of Ward Colorado, [40.069261 N, 105.584835 W], 21.viii.1977, 1 male, USNM ENT 258062, A.G. Lavallee (USNM); Brainard Lake Rec. Area 4mi W of Ward Colorado, [40.069261 N, -105.584835 W], 21.viii.1977, 1 male, USNM ENT 258063, A.G. Lavallee (USNM); Cameron Pass, [40.520816 N, -118.227668 W], 28.vii.1954, 1 male, OSAC 133386, Chas. L. Fluke (OSAC); North Park, [40.73 N, -106.18 W], 1.vii.1932, 1 female, JSS 20665, (CSUC); Brainard Lake, 11.viii.1952, 1 female, JSS 21103, R.R. Dreisbach (MSUC); Washington: Mt. Ranier, Paradise Valley, [46.782709 N, -121.727952 W], 15.vii.1920, 1 male, JSS 23880, E.C. Van Dyke (CAS); Mt. Ranier, Paradise Vallley, [46.782709 N, -121.727952 W], 18.vii.1920, 7 males, JSS 23722, 23888, 23889, 23893, 23894, 23900, 23904, E.C. Van Dyke (CAS); Mt. Ranier, Paradise Valley, [46.782709 N, -121.727952 W], 19.vii.1920, 1 male, JSS 23887, 1 female, JSS 23718, E.C. Van Dyke (CAS); Mt. Ranier, Paradise Vallley, [46.782709 N, -121.727952 W], 25.vii.1920, 1 male, JSS 23883, E.C. Van Dyke (CAS); Mount Rainier National Park, Carbon River near Ranger station, [46.85 N, -121.75 W], 15.viii.1999, 1 female, ISS 20681, B. Kondratieff (CSUC); Mt. Rainier NP Louis Lake, Fryingpan Cr., Sunrise Rd., 
[46.85 N, -121.75 W], 17.vi.2004, 1 male, JSS 20804, B. Kondratieff, J. Schmidt, J.

Owens (CSUC); Paradise Valley, Mount Rainier, [46.85 N, -121.75 W], 18.vii.1920, 1 male, JSS 20613, E.C. Van Dyke (EMEC); Seattle, [47.60621 N, -122.332071 W], 16.vi.1901, 1 female, OSAC 133444, (OSAC); Mt. Baker-Snoqualmie National Forest: Mt. Baker: Picture Lake, [48.866217 N, -121.676533 W], 1273m, 7.vii.2010, 1 male, JSS 23706, 1 female, JSS 24625, A. Young (DEBU); Mount Baker, Snoqualmie National Forest, Silver Fir Campground, [48.90495 N, 121.697917 W], 613m, 9.vii.2010, CNCD 60043, M.M. Locke (CNC); Mount Baker, Snoqualmie National Forest, Silver Fir Campground, [48.90495 N, 121.697917 W], 613m, 9.vii.2010, CNCD 60046, CNCD 60047, A. Young (CNC); Mt. Baker-Snoqualmie National Forest; Mt. Baker; Silver Fir Campground, [48.90495 N, -121.697917 W], 8.vii.2010, 1 male, JSS 23708, M.M. Locke (CNC); Mt. Baker-Snoqualmie National Forest; Mt. Baker; Silver Fir Campground, [48.90495 N, -121.697917 W], 9.vii.2010, 1 female, CNCD 35311, M.M. Locke (CNC); Paradise Val., 19.vii.1920, 1 male, JSS 23886, E.C. Van Dyke (CAS); Wyoming: Wyoming, [43.084281 N, -107.556417 W], 1952, 1 female, CNCD 26329, F.M. Hull (CNC); Yellowstone National Park, [44.587719 N, 110.537673 W], 20.vii.1925, 1 male, CNCD 26343, A.A. Nichol (CNC).

\section{Dasysyrphus pacificus (Lovett)}

Figures: 10D, E, 15B \& 24

Synonyms and References: 
Syrphus pacifica Lovett, 1919: 245 (in (Cole \& Lovett 1919); original description) Metasyrphus pacifica (Lovett):

Fluke, 1933: 116 (key, redescription and transfer to Metasyrphus) Dasysyrphus pacificus (Lovett):

Stone et al., 1965: 564 (catalogue and transfer to Dasysyrphus) Dasysyrphus pauxillus (Williston):

Vockeroth, 1986: 203 (in part; synonymy)

Vockeroth, 1992: 68 (in part; key and redescription)

Diagnosis: Abdominal maculae of tergites $3 \& 4$ slightly arcuate, uniform in thickness and do not cross abdominal margin (Figs 10D, E); very similar in appearance to $D$. pauxillus (Fig. 11A), D. nigricornis (Figs 7A, C) and D. laticaudus (Fig. 7E). Wing typically much more bare than any of the three aforementioned species, with cells $\mathrm{br}$ and bm with large dense areas. Male gonostylus with narrow, wavy projection on posterodorsal surface (Fig. 15B). Due to an extremely similar habitus, it is difficult to distinguish $D$. pacificus from $D$. pauxillus. In the contact zone between these species in southern British Columbia male genitalia should be used to correctly identify specimens.

Description:

Body Length: $5.0-8.5 \mathrm{~mm}$; Wing Length: $5.0-7.7 \mathrm{~mm}$ 
Head: Frons dark with light pollenose fascia (in males it runs along ventral edge of where eyes meet, in females it is $1 / 2$ the length between the antennae and ocelli) and dark pile; face light with dark vitta $1 / 4$ the width of the face, reaching antennal socket, face with dark pile; gena dark with some light pollen ventral to eye, dark pile dorsally and light pile ventrally; occiput dark, covered in light pollen, pile light with some dark pile dorsally; scape; pedicel and flagellomere light to dark.

Thorax: Scutum dark, sometimes shiny or metallic, pile light; scutellum light to dark with darker anterior and lateral edges, light pile with some dark pile posteriorly; wing cell br bare on basal half and above spurious vein, bm bare on basal half, cells $c$ and $r_{1}$ bare at base or with at least small bare areas at base, cup call has small bare areas along vein $\mathrm{CuP}$; haltere light to dark; femora basally dark, apically light, tibiae light, metatibia sometimes with dark band close to apex, tarsi light with darker anterior side.

Abdomen: Maculae on tergite 2 large, slender and oval, $1 / 3-1 / 2$ width of tergite, maculae on tergites $3 \& 4$ are slightly arcuate, uniform in thickness, with out a distinct swelling medially, maculae do not reach abdominal margin (Figs $10 \mathrm{E}, \mathrm{D}$ ); sternites light with dark fasciae.

Male Genitalia: Surstylus more or less triangular in shape in lateral view, flattened posteriorly, long pile on dorsal, laterodorsal and posterodorsa! sides, row of spines on posteriomedial edge; cercus oval with long pile over entire surface; gonostylus 
triangular-shaped with narrow, wavy projection on posterodorsal surface projecting posterodorsally, pile on dorsal surface; basiphallus elongate, basally bent at 90 degree angle towards dorsum, apical end curved ventrally with a widened apex and is partly membranous dorsally, with spines on posterodorsal side; distiphallus elongate, apical end flared out into horn, which is mostly sclerotized but membranous at apex with few minute setulae dorsally, basally bent towards dorsum and usually greatly enlarged at bend with a triangular projection, projecting ventrally (Fig. 15B).

Intraspecific variation: Distiphallus sometimes without triangular projection basally. Abdomenal maculae on tergites $3 \& 4$ rarely meet medially, and if so, barely touch.

Etymology: Pacificus is Latin for peaceful or peace-making, but more probably was named so because this species is found along the Pacific coast of North America.

Distribution: Southwestern USA (CA, NV, OR, WA) and southwestern Canada (BC); Cascades and Sierra Nevada Mountain Ranges (Fig. 24).

Ecology:

Collected from April-July, but more commonly in May and June, at elevations of 4753100m. Recorded from willow (Salix, Salicaceae) and bitterbush (Purshia de Candolle ex Poiret, Rosaceae). 
Discussion: Dasysyrphus pacificus is hereby resurrected from synonymy with Dasysyrphus pauxillus (synonymized by Vockeroth (1986)). The type specimen of this species could not be examined as it is out on loan to another individual. However, based on the written description and distribution of D. pacificus (Cole \& Lovett 1919) the author is confident that the individuals examined were of this species. The range of pacificus is from southern British Columbia, south to California, in the Cascades and Sierra Nevada mountain ranges. The type specimen is from Oregon, where it is the only species of the pauxillus s.l. in this range.

Type Material: "Holotype (No. 502), male, in Museum of the California Academy of Sciences, collected at Corvallis, Ore., May 17, by A. L. Lovett" (Cole \& Lovett 1919). (not examined)

Material Examined:

Canada: British Columbia: Bear Mountain, [49.300555 N, -121.740556 W], 6.v.1920, 1 male, CNCD 13743, (CNC); Cranbrook, East Kootenay R.D., [49.517983 N, -115.761504 W], 19.v.1920, 1 male, CNCD 13748, C.B.D. Garrett (CNC); Cranbrook, [49.517983 N, -115.761504 W], 27.iv. 1 male, Royal British Columbia Museum ENT 99188108, H.R. Foxlee (RBCM); 7 miles N Lions Bay, North of Vancouver, 18.v.1979, 1 male, CNCD 5464, G.F. Hevel (USNM); United States of America: California: Riverside Co.[unty], Dark Canyon N of Idyllwild, [33.81666667 N, -116.7166667 W], 24.v.2003, CNCD 8472, J. Skevington (CNC); 
Upper Santa Ana River, San Bernardino County, [34.091253 N, -117.120741 W], 3.vi.1947, 1 female, CNCD 26415, G.H. \& J.L. Sperry (CNC); 1 km NW Wrightwood, Big Pines Hwy, [34.38138889 N, -117.6716667 W], 1974m, 21-28.v.2005, 5 males, JSS 20974, 20976-20978, 20980, 2 females, JSS 20975, 20979, A.R. Cline \& S.L. Winterton (MHPC); Oakhurst, [37.328 N, -119.649315 W], 1.vi.1942, 1 female, JSS 20598, E.C. Linsley (EMEC); Mather Site, Buck Meadows, Tuolumne County, [37.880173 N, -119.856977 W], 1970, 1 male, CNCD 26437, 4 females, CNCD 2640426407, A.R. Moldenke (CNC); Lily Pond, Alpine Lake, Marin Co., [37.953803 N, 122.634977 W], 457m, 11.iv.-25.v.1971, 1 male, CNCD 26344, D.D. Munroe (CNC); Lily Pond, Alpine Lake, Marin Co., [37.953803 N, -122.634977 W], 25.v.1970, 1 female, CNCD 26414, D.D. Munroe (CNC); Calaveras Big Trees SP, S. grove, banks of Beaver Creek, MT \#2, [38.26166667 N, -120.2577778 W], 1375m, 22.v.-11.vi.2007, 6 females, JSS 20966-20970, 20972,A.R. Cline \& P.H. Kerr (MHPC); Indian Grinding Rock St. Pk. dry wash nr. S. Nature Trail MT \#2, [38.41666667 N, -120.6333333 W], 715m, 5.iv.-24.iv.2007, JSS 20981, 1 female, ISS 20992, P. Kerr \& M. Hauser (MHPC); Indian Grinding Rock St. Pk. firebreak nr. envtl camp MT3, [38.41861111 N, 120.635 W], 715m, 24.iv.2007 24.v.2007, 1 female, JSS 20971, P. Kerr \& M. Hauser (MHPC); Sonora Pass, [38.53666667 N, -119.6855556 W], 3100m, 21.vi.2007, 1 female, JSS 20963, S.M. Blank (MHPC); Wright's Lake, [38.84913 N, -120.2318 W], 2.vii.1948, 1 male, JSS 20597, D. Carter (EMEC); Wright's Lake, [38.84913 N, 120.2318 W], 2.vii.1948, 1 female, JSS 20601, L.W. Quate (EMEC); Placer County, [39.090423 N, -120.803069 W], 1524m, iv, 1 female, JSS 19511, W.M. Davidson (CNC); Tahoe City, Alpine Meadow Road Jenkinson Reservoir, [39.42138889 N, - 
120.3333333 W], 2200m, 14.vi.2007, 1 male, JSS 20957, S.M. Blank (MHPC); Tahoe City, Alpine Meadow Road Jenkinson Reservoir, [39.42138889 N, -120.3333333 W], 2200m, 14.vi.2007, 1 female, JSS 20958, S.M. Blank (MHPC); Sinkyone Wilderness State Park, [39.83583333 N, 123 W], 28m, 23.iii.2003, 1 male, JSS 20797, J. \& A. Skevington (CNC); Buck's Lake, Plumas County, [39.890961 N, -121.185778 W], 13.v.1949, 1 female, CNCD 26409, P.D. Hurd (CNC); Buck's Lake, Plumas County, [39.890961 N, -121.185778 W], 13.v.1949, 1 male, CNCD 26432, 1 female, CNCD 26410, W.W. Middlekauff (CNC); Buck's Lake, Plumas County, [39.890961 N, 121.185778 W], 23.vi.1949, 2 females, CNCD 26412, D. Cox (CNC); Buck's Lake, Plumas County, [39.890961 N, -121.185778 W], 23.vi.1949, 1 female, CNCD 26413, J.W. MacSwain (CNC); Buck's Lake, Plumas County, [39.890961 N, -121.185778 W], 13.v.1949, 3 males, CNCD 26433-26435, W.W. Middlekauff (CNC); Buck's Lake, Plumas County, [39.890961 N, -121.185778 W], 13.v.1949, 1 male, JSS 20594, E.0. Essig (EMEC);

Buck's Lake, Plumas County, [39.890961 N, -121.185778 W], 13.v.1949, 1 male, JSS 20595, P.D. Hurd (EMEC); Buck's Lake, Plumas County, [39.890961 N, -121.185778 W], 23.vi.1949, 1 female, JSS 20599, J.W. MacSwain (EMEC); Buck's Lake, Plumas County, [39.890961 N, -121.185778 W], 23.vi.1949, 1 female, ISS 20600, J.W. MacSwain (EMEC); Buck's Lake, Plumas County, [39.890961 N, -121.185778 W], 13.v.1949, 1 female, JSS 20603, P.D. Hurd (EMEC); 60 kilometers North East of Chico on Road 32, Tehama County, [40.19611111 N, -121.5213889 W], 1100m, 15.v.2010, ISS 25002, 25003, 25005-25009, D. Striley, M. Hauser (CSCA); Susan River Campground, Lassen County, [40.492316 N, -121.094484 W], 9.vii.1949, 1 female, 
CNCD 26411, A.T. McClay (CNC); Viola, Shasta County, [40.517921 N, -121.680971 W], 19.v.1941, 1 male, CNCD 26436, 1 female, CNCD 26408, E.G. Linsley (CNC); Manzanita Lake, Lassen National Park, [40.534513 N, 121.56655 W], 8.vi.1941, 1 female, JSS 20596, C.D. Michener (EMEC); Manzanita Lake, Lassen National Park, [40.534513 N, 121.56655 W], 8.vi.1941, 1 female, JSS 20602, Arthur J Walz (EMEC); Ryan Creek, 17.v.1938, 1 female, CNCD 106796, N.F. Hardman (CNC); San Bernardino Mountains; Up Sta. Ana River, 15.vi.1955, 1 female, JSS 23775, A.L. Melander (USNM); Up Sta Ana Riv, 1957, 1 female, JSS 26926, A.L. Melander (USNM); Nevada: Little Valley, Washoe County, [39.806851 N, -119.951585 W], 4.vi.1981, JSS 25100, R.W. Rust (EMUS); Oregon: Lake of the Woods, Klamath County, [42.368634 N, -122.208482 W], 22.vi.1975, JSS 25082-25086, 2508825095, G.F. Knowlton (EMUS); Summit Lake, Klamath County, [43.454905 N, 122.118766 W], 7.vii.1952, 1 male, CNCD 26442, E.R. Jaycox (CNC); Camp Abbot, Deschutes County, [43.874555 N, -121.435449 W], 4.vi.1944, 1 female, CNCD 26401, P.H. Arnaud (CNC); Bellfountain, [44.363611 N, -123.355278 W], 27.v.1922, 1 male, OSAC 133389, 133391, A.L. Lovett (OSAC); Corvallis, Benton County, [44.564564 N, -123.262033 W], v.1977, 1 male, JSS 19507, A.L. Lovett (CNC); Klamath Co., Lake of the Woods, 22.vi.1975, 1 female, CNCD 5457, G.F. Knowlton (USNM); Washington: Wenatche National Forest, White Pass Trail, [46.643317 N, 121.378783 W], 1345m, 6.vii.2010, CNCD 60054, A. Young (CNC); Wenatche National Forest; White Pass Trail, [46.643317 N, -121.378783 W], 6.vii.2010, 1 male, CNCD 35314, 1 female, CNCD 35313, M.M. Locke (CNC); Mount Rainier, [46.853048 N, -121.753127 W], 1 male, CNCD 26429, 1 female, CNCD 26400, 26419, 
F.M. Hull (CNC); Olga, San Juan County, [48.619548 N, -122.835459 W], 18.v.1910, 1 female, JSS 19504, (CNC); Mount Baker, Snoqualmie National Forest, Picture Lake, [48.866217 N, $121.676533 \mathrm{~W}], 1255 \mathrm{~m}, 8 . v i i .2010$, CNCD 60058, A. Young (CNC); Pleasant Valley, Price County, [48.90667 N, -122.70333 W], 21.v.1977, ISS 25097, T.L. Whitworth (EMUS).

\section{Dasysyrphus pauxillus (Williston)}

Figures: $11 \mathrm{~A}, \mathrm{~B}, 16 \mathrm{~A}, 25$

Synonyms and References:

Syrphus pauxillus Williston, 1887: 74 (original description)

Metasyrphus pauxillus:

Fluke, 1933: 115 (key, redescription and transfer to Metasyrphus) Dasysyrphus pauxillus:

Stone et al., 1965: 564 (catalogue and transfer to Dasysyrphus)

Vockeroth, 1986: 203 (discussion)

Vockeroth, 1992: 68 (key and redescription)

Speight, 2011: 70 (discussion)

Diagnosis: Abdominal maculae of tergites $3 \& 4$ slightly arcuate, uniform in thickness and do not cross abdominal margin (Figs 11A, B); very similar in appearance to $D$. pacificus (Fig. 10D), D. nigricornis (Figs 7A, C) and D. laticaudus (Fig. 7E). Wing 
typically densely microtrichose, sometimes with bare areas in cells $r_{1}, b r, b m$ and/or c. Male gonostylus with projection on posterodorsal surface straight (Fig. 16A). Due to an extremely similar habitus, it is difficult to distinguish D. pacificus from D. pauxillus. In the contact zone between these species in southern British Columbia, male genitalia should be used to correctly identify specimens.

\section{Description:}

Body Length: $5.3-9.7 \mathrm{~mm}$; Wing Length: $5.2-9.5 \mathrm{~mm}$

Head: Frons dark with light pollenose fascia (in mäles it runs along ventral edge of where eyes meet, in females it is $1 / 3-1 / 2$ the length between the antennae and ocelli with a separation medially and edges triangular to rounded) and light to dark pile; face light with dark vitta $1 / 4-1 / 3$ the width of the face, coming close to antennal socket if not reaching it, face with dark pile lateral to the eye, some females with varying amounts of light pile interspersed with dark pile; gena with light to dark pile; occiput dark, covered in light pollen, pile light; scape and pedicel light to dark, flagellomere dark with lighter base.

Thorax: Scutum dark, sometime shiny or metallic, pile light to dark; scutellum light to dark with darker lateral edges, pile on female mostly light with some dark, male mostly dark pile some with light interspersed throughout; wing cel! $r_{1}$ with the base bare or very small areas at base bare, br, bm and c may all be entirely microtrichose 
or they may have bare areas along spurious vein, along the middle of the cell form the base to $3 / 4$ or small areas at base, respectively; haltere light; femora basally dark, apically light, tibiae light, meso- and metatibia sometimes with dark band close to apex, tarsi light with darker anterior side.

Abdomen: Maculae on tergite 2 large and oval, lateral edge tends to be more flattened than medial edge, female usually with very small, pointed, anterolateral projection, but it never reaches the edge of the tergite, maculae on tergites $3 \& 4$ are oblique, fairly straight and uniform in width, sometimes with medial edge slightly swollen, maculae do not reach abdominal margin (Figs 11A, B); sternites light with dark fasciae of varying thickness.

Male Genitalia: Surstylus more or less triangular in shape in lateral view, flattened posteriorly, long pile on dorsal, laterodorsal and posterodorsal sides, row of spines on posteriomedial edge; cercus oval with long pile over entire surface; gonostylus boot-shaped but more narrow apically than basally, with projection on posterodorsal surface, projecting dorsally, pile on dorsal surface; basiphallus elongate, basally bent at a slightly more than 90 degree angle towards dorsum, apical end curved ventrally, but does not greatly extend ventrally and is partly membranous dorsally, with spines on posterodorsal side; distiphallus elongate, apical end flared out into horn, which is mostly sclerotized but membranous at apex, with no setulae dorsally, basally bent towards dorsum and usually greatly enlarged at bend with a triangular projection, projecting ventrally (Fig. 16A). 
Extremely similar to D. laticaudus (Fig. 15A).

Intraspecific variation: Wings are typically densely microtrichose with cell $r_{1}$ having bare areas at base ranging from entire base bare to very small areas at base being bare, cells br, bm and c may all be entirely microtrichose or they may have bare areas; female usually have a very small, anterolateral projection on maculae of tergite 2, males do not have this projection.

Etymology: From the Latin pauxillus, meaning small

Distribution: Rocky Mountains; Western Canada (AB, BC, MB, YT) and western USA (AK, CO, ID, NM, UT, WY) (Fig. 25).

Ecology: Collected from March - July, but more commonly collected in June. Collected at elevations of $14-3505 \mathrm{~m}$. Habitats collected from are listed as "moraine lake", "grassy lakeshore", "over stream in fir forest", "on tundra", "spruce forest" and "Pinus-Salix forest". It has been collected from the following plant species: common cow parsnip (Heracleum maximum, Apiaceae), blackberry (Rubus, Rosaceae), willow (Salix, Salicaceae), Canada gooseberry (Ribes oxyacanthoides Linnaeus, Grossulariaceae) and spruce (Picea A. Dietrich, Pinaceae).

Discussion: Many authors have used the name pauxillus in the Palaearctic. During the course of this revision few specimens from that region were examined and no 
DNA evidence could be studied. Therefore, I cannot comment on whether the use of this name is appropriate in the Palaearctic. However, based on the geographic breakdown of pauxillus and its allies (laticaudus, pacificus and nigricornis) in the Nearctic it seems likely that only nigricornis is Holarctic. European and Asian specimens should be studied in greater depth, using both morphology and DNA, to confirm this suspicion.

Due to the widespread use of the name pauxillus much confusion exists over the concept of this species. Many Holarctic species have been named which have an extremely similar habitus to pauxillus. The main reason for such confusion is that the holotype specimen is lost (Fluke, 1933). In Williston's (1887) original designation he describes one female from New Mexico. He does not indicate where this specimen is deposited. The only other information that is provided is that the specimen was collected by G.F. Gaumer. In Fluke's (1933) revision of Metasyrphus he indicates that he was unable to locate the type for examination. No record exists in the literature of any author examining Williston's specimen; either Williston's species description was used or specimens determined by other researchers were used for comparison. Dasysyrphus pauxillus was described in 'Synopsis of the North American Syrphidae', Williston's PhD dissertation (Beckemeyer 2009). The specimens from his dissertation were donated to the USNM (Flint et al. 1976, F.C. Thompson, pers. comm.). According to Thompson (pers. comm.) the type specimen is not in the USNM collection today and it is not known what happened to it. Williston worked in Kansas and Chicago until his death, after which his collection 
was donated to the American Museum of Natural History (AMNH). Byers et al. (Byers et al. 1962) do not list pauxillus as a type in the Kansas collection and the specimen is not listed in the type database of the AMNH (The American Museum of Natural History 2005). A neotype is designated here to bring stability to the name pauxillus. This will create a solid concept for this species, allowing for better reconciliation of closely related species. The specimen used in the original designation provided a locality of 'New Mexico', so the neotype specimen was chosen from this state. This is a locality that is not overlapped by the other species with a similar habitus to pauxillus (pacificus, laticaudus and nigricornis). The neotype varies from Williston's description in a few characters that are variable in many Dasysyrphus species. The facial stripe of the neotype closely approximates the antennae; however, this character is variable in this species. The scutellum in the neotype has a larger yellow patch than indicated by Williston; again this character is variable and the scutellum ranges from yellow to largely brown within the species. The face is also not projecting to the extent described by Williston. The face seems to project slightly more in females than males, but in most specimens the distance between the antennae and the tip of the tubercle is more or less equal to the distance from the antennae to the ocelli. The specimen matches the description in all other characters. The Neotype is a male from Sierra Blanca, Lincoln Co, NM, USA, collected on 10-26.vi.1979 by By: S. \& J. Peck. It is deposited in the CNC and databased with the number Jeff Skevington Specimen 18307. 
Type Material: "NM, Lincoln Co./ Sr. Blanca 9700ft./ 10-26.VI.1979/ S. \& J. Peck”.

"Dasysyrphus pauxillus (Will.)/ Det. J.R. Vockeroth", "J. Skevington/ Specimen \#/ 18307", "NEOTYPE/ Dasysyrphus / pauxillus (Will.)/ Det. M.M. Locke"; male, deposited in the Canadian National Collection of Insects, Arachnids and Nematodes, Ottawa, Ontario, Canada.

Material Examined:

Canada: Alberta: Waterton, Waterton Lakes National Park, [49.051122 N, 113.911807 W], 6.vi.1962, 2 males, CNCD 13764, 13765, 4 females, CNCD 1383613839, W.R.M. Mason (CNC); Waterton, Waterton Lakes National Park, [49.051122 N, -113.911807 W], 6.vi.1962, 2 females, CNCD 13834, 13835, K.C. Herrmann (CNC); Kananaskis 25 km WNW Bragg Creek Powderface trail Rd., [51.02166667 N, 114.9016667 W], 8.vi.2001, 1 male, Royal British Columbia Museum ENT 6006051, C.J. Borkent (RBCM); 30 miles West of Calgary, [51.04575 N, $-113.631333 \mathrm{~W}$, 3.vi.1973, CNCD 60100, G.G.E. Scudder (CNC); Kananaskis, Forest Experimental Station Seebe, [51.100879 N, -115.087692 W], 2.vi.1968, 1 female, CNCD 13840, H.J. Teskey (CNC); Kananaskis, Forest Experimental Station Seebe, [51.100879 N, 115.087692 W], 13.vi.1968, 1 female, CNCD 13841, H.J. Teskey (CNC); Kananaskis, Forest Experimental Station Seebe, [51.100879 N, -115.087692 W], 15.vi.1968, 1 female, CNCD 13842, H.J. Teskey (CNC); Banff, Banff National Park, [51.180275 N, 115.568433 W], 27.v.1925, 1 male, CNCD 13762, E. Hearle (CNC); Banff, Banff National Park, [51.180275 N, -115.568433 W], 2.vi.1922, 1 male, CNCD 13763, 3 females, CNCD 13845-13847, C.B.D. Garrett (CNC); Banff, Banff National Park, 
[51.180275 N, -115.568433 W], 3.vii.1922, 1 female, CNCD 13848, C.B.D. Garrett (CNC); Banff, Banff National Park, [51.180275 N, -115.568433 W], 9.vi.1922, 1 female, CNCD 13849, C.B.D. Garrett (CNC); Banff, [51.180275 N, -115.568433 W], 2.vi.1914, 1 female, JSS 20605, N.B. Sanson (EMEC); Banff, [51.180275 N, 115.568433 W], 2.vi.1948, 1 female, UASM\# 149025, E.H. Strickland (UASM ); Banff, [51.180275 N, -115.568433 W], 30.v.1948, 1 female, UASM\# 149024, E.H. Strickland ( UASM ); Mount Eisenhower, Banff National Park, [51.298694 N, 115.925724 W], 7.vi.1968, 1 male, CNCD 13768, Mosquin \& Seaborn (CNC); Mount Eisenhower, Banff National Park, [51.298694 N, -115.925724 W], 5.vi.1968, 1 male, CNCD 13769, Mosquin \& Seaborn (CNC); Mount Eisenhower, Banff National Park, [51.298694 N, -115.925724 W], 3.vi.1968, 2 males, CNCD 13770, 13771, Mosquin \& Seaborn (CNC); Moraine Lake, Banff National Park, [51.324073 N, -116.184418 W], 2256m, 20.vii.1955, 1 female, CNCD 13850, J.R. McGillis (CNC); 8 km West of Lake Louise, [51.413684 N, -116.306046 W], 31.v.1992, 1 female, JSS 20801, A. Borkent (CNC); Lake Agnes, Banff National Park, [51.415101 N, -116.247314 W], 2073m, 10.vii.1925, 1 male, JSS 19508, O. Bryant (CNC); Snow Creek Pass, Banff National Park, [51.60524 N, -115.808617 W], 2256m, 20.vii.1962, 1 female, CNCD 13844, K.C. Herrmann (CNC); Peyto Lookout, Banff National Park, [51.717631 N, -116.508919 W], 2316m, 23.vii.1962, 1 female, CNCD 13843, W.R.M. Mason (CNC); Edmonton, 12.v.1924, 1 male, JSS 23724, Owen Bryant (CAS); Laggen, Lake Agnes, 10.vii.1925, 1 female, JSS 23714, O. Bryant (CAS); Edmonton; W. Hawrelak Park, 640m, 3.v.2005, 3 males, ISS 25540-25542, W. van Steenis (WVSC); Pincher Creek; Beauvais Lake, 19.v.1989 23.v.1989, 1 female, JSS 25550, C. v. Nidek (WVSC); British Columbia: 
Victoria, Mt. Work, [48.53 N, -123.47 W], 26.v.1982, 1 male, Royal British Columbia Museum ENT 99188111, R.A. Cannings (RBCM); Galiano I. North End, [48.92 N, 123.42 W], 29.iii.1987, 1 male, JSS 20184, G.G.E. Scudder (UBCZ); Cameron Lake, Waterton Lakes National Park, Division No. 3, [49.011457 N, -114.049238 W], 17.vi.1956, 9 males, CNCD 13754-13761, 13767, 9 females, CNCD 13822-13830, E.E. Sterns (CNC); Cameron Lake, Waterton Lakes National Park, Division No. 3, [49.011457 N, -114.049238 W], 18.vi.1956, 1 female, CNCD 13831, E.E. Sterns (CNC); Cameron Lake, Waterton Lakes National Park, Division No. 3, [49.011457 N, -114.049238 W], 19.vi.1956, 2 females, CNCD 13832, 13833, E.E. Sterns (CNC); Robson, [49.333015 N, -117.692871 W], 25.iv.1958, 1 male, JSS 20123, H.R. Foxlee (UBCZ); Robson, Central Kootenay L.D., [49.342029 N, -117.697831 W], 21.iv.1947, 1 male, CNCD 13746, H.R. Foxlee (CNC); Robson, Central Kootenay L.D., [49.342029 N, -117.697831 W], 8.v.1950, 1 male, CNCD 13750, H.R. Foxlee (CNC); Boswell, Central Kootenay A, [49.414407 N, -116.746741 W], 9.v.1958, 1 male, CNCD 13742, H. \& A. Howden (CNC); Meager Creek Hot Springs, Squamish-Lillooet R.D., [50.561617 N, -123.486344 W], 14.v.1987, 1 male, JSS 18370, C.S. Guppy (CNC); Kootenay National Park, Daer-Pitts Aspen Control 1, [50.95 N, -116.0333333 W], 3.vi.2000 17.vi.2000, CNCD 7969, G. Gareau (CNC); 32 miles South West of Terrace, [54.194076 N, -129.140296 W], 30.5m, 11.vi.1960; 1 male, CNCD 13753, B. Heming (CNC); 32 miles South West of Terrace, [54.194076 N, -129.140296 W], 6.vi.1960, 1 female, CNCD 13819, G.E. Shewell (CNC); 32 miles South West of Terrace, [54.194076 $\mathrm{N},-129.140296 \mathrm{~W}], 30.5 \mathrm{~m}, 6 . \mathrm{vi} .1960,1$ female, CNCD 13820, B. Heming (CNC); Shames, 18 Miles South West of Terrace, [54.409648 N, -128.935301 W], 
23.vi.1960, 1 female, CNCD 13818, C. H. Mann (CNC); Kleanza Creek, Near Terrace, [54.597897 N, -128.386241 W], 76.2m, 29.vi.1960, 1 female, CNCD 13817, B. Heming (CNC); Kleanza Creek, Near Terrace, [54.597897 N, -128.386241 W], 30.vi.1960, 1 female, CNCD 13821, J.G. Chillcott (CNC); Atlin, [59.5775 N, 133.69236 W], 670.56m, 2.vi.1955, 1 male, CNCD 13751, H. Huckel (CNC); Atlin, [59.5775.N, -133.69236 W], 670.56m, 20.vi.1955, 1 male, CNCD 14358, 1 female, CNCD 13808, B.A. Gibbard (CNC); Atlin, [59.5775 N, -133.69236 W], 670.56m, 4.vii.1955, 1 female, CNCD 13809, H.J. Huckel (CNC); Kootenay National Park, DaerPitts Aspen Control 2, 17.vi.2000 2.vii.2000, 1 female, CNCD 30906, G. Gareau (CNC); Manitoba: 26km SE Churchill; Twin Lakes, [58.63 N, -93.819 W], 14m, 22.vii.2009, 1 male, 09PROBE 255, Arctic Ecology 2009 (CNC); 26km SE Churchill; Twin Lakes, [58.63 N, -93.819 W], 14m, 19.vii.2009, 1 female, 09PROBE 197, Arctic Ecology 2009 (CNC); Yukon Territory: Snafu Creekl, [60.13333333 N, -138.8 W], 22.vi.1982, CNCD 60079, S.G. Cannings,R.D. Wilkie, L. Vasington (CNC); Whitehorse, [60.71965 N, -135.052213 W], 6.vii.1988, 1 female, CNCD 5812, F. Brodo (CNC); Simpson L. $81 \mathrm{~km} n$ Watson L., [60.75 N, -129.25 W], 15.vi.1984 1.ix.1984, 1 female, JSS 20770, S. \& J. Peck (CNC); Nines Creek, 3 km West of Alaska Highway, [61.18333333 N, -139.7 W], 4.vi.1981, CNCD 60072, C. Guppy (CNC); La Force Lake, [62.683333 N, -132.333333 W], 914m, 26.vi.1960, 1 female, CNCD 13585, E.W. Rockburne (CNC); La Force Lake, [62.683333 N, -132.333333 W], 1006m, 10.vii.1960, 1 female, CNCD 13728, E.W. Rockburne (CNC); United States of America: Alaska: Matanuska, Matanuska-Susitna County, [61.541945 N, 149.229674 W], 12.vi.1944, 1 female, JSS 19502, J. Chamberlin (CNC); Colorado: 
Rocky Gulch, [37.817035 N, -107.579606 W], 3444m, 6.xxii.2002, 1 male, JSS 20810, C. Slater (CSUC); Monarch Pass, 2mi W Garfield, [38.501433 N, -106.327747 W], 3048m, 19.vi.1985, 1 male, USNM ENT 258074, David R. Smith (USNM); Rock Cr., May Mus., [38.705655 N, -104.837421 W], 8.v.1996, 1 female, ISS 20808, D. Leatherman (CSUC); Tenmile Range, unamed Cr. ayalanche chute W side, [39.425 N, -106.111 W], 3200m, 15.vii.1998 17.vii.1998, 1 male, JSS 20694, S. Fitzgerald \& A. Foley (CSUC); [39.550051 N, -105.782067 W], 1 male, JSS 20745, (CSUC); Arapaho National Forest, Mt. Evans, Echo Lake Campground, [39.65622 N, 105.595042 W], 329m, 29.vi.2010, CNCD 60035, M.M. Locke (CNC); Echo Lake, Mt. Évans, Clear Creek County, [39.658085 N, -105.6035 W], 3231m, 13.vii.1961, 1 female, CNCD 26416, C.H. Mann (CNC); Arapaho National Forest, Mt. Evans, Echo Lake, Path on west side of lake, [39.659767 N, -105.604767 W], 3176m, 29.vi.2010, 1 female, JSS 24618, M.M. Locke (CNC); Arapaho National Forest; Mt. Evans; Echo Lake, [39.659767 N, -105.604767 W], 29.vi.2010, 1 female, CNCD 35312, A. Young (CNC); Doolittle Ranch, Mt. Evans, Clear Creek County, [39.675739 N, -105.601348 W], 2987m, 13.vii.1961, 1 female, CNCD 26417, W.R.M. Mason (CNC); Nederland, Science Lodge, Boulder County, [40.031645 N, -105.533886 W], 2896m, 4.vii.1961, 1 female, CNCD 26418, B.H. Poole (CNC); Niwot Ridge, Near Ward, Boulder County, [40.069933 N, -105.607397 W], 3444m, 28.vi.1961, 1 male, CNCD 26439, C.H. Mann (CNC); Niwot Ridge, Near Ward, Boulder County, [40.069933 N, -105.607397 W], 3505m, 4.vi.1961, 1 male, CNCD 26440, C.H. Mann (CNC); North Park, [40.73 N, 106.18 W], 1.vii.1932, 1 female, ISS 20746, (CSUC); Rawah Wilderness, Blue Lake Trail near Fall Creek, [40.76944444 N, -105.9894444 W], 2926m, 20.vi.1998, 1 
male, JSS 20693, S. Fitzgerald \& A. Foley (CSUC); Cherokee Park, [40.90972 N, 105.47917 W], 30.vii.1913, 1 female, JSS 20713, Chas L. Fluke (CSUC); Continental Divide, Mineral County, 3292m, 20.vi.1919, 1 male, JSS 19512, (CNC); Camera Point, Pike's Peak, 16.vi.1956, 1 female, JSS 21157, R. \& K. Dreisbach (MSUC); Idaho: Island Park, [44.424364 N, -111.371064 W], JSS 25099, Bernard Knapp (EMUS); Lake Waha, Nez Perce County, [46.205942 N, -116.837015 W], 18.vi.1930, 1 male, ISS 19514, W.M. Davidson (CNC); Lake Waha, Nez Perce County, [46.206824 N, 116.835552 W], 9.vi.1918, 1 female, CNCD 26402, A.L. Melander (CNC); Waha, Nez Perce County, [46.211439 N, -116.844439 W], 18.vi.1909, 1 female, JSS 19501, (CNC); Moscow Mt., [46.800972 N, -116.866093 W], 8.vi.1921, 1 female, JSS 23776, A.L. Melander (USNM); Moscow Mt., [46.800972 N, -116.866093 W], 26.vi.1922, 1 female, JSS 23777, A.L. Melander (USNM); Mountains Near Moscow, Latah County, [46.807258 N, -116.868969 W], 1.vi.1907, 1 female, CNCD 26403, (CNC); Mountains Near Moscow, Latah County, [46.807258 N, -116.868969 W], 9.vii.1920, 1 female, ISS 19505, A.L. Melander (CNC); Mountains Near Moscow, Latah County, [46.807258 N, -116.868969 W], 26.vi.1920, 1 male, JSS 19506, A.L. Melander (CNC); Mountains Near Moscow, Latah County, [46.807258 N, -116.868969 W], 17.vi.1918, 1 male, JSS 19509, A.L. Melander (CNC); Mountains Near Moscow, Latah County, [46.807258 N, -116.868969 W], vii.1910, 1 female, JSS 19510, R.C. Shannon (CNC); Mountains Near Moscow, Latah County, [46.807258 N, -116.868969 W], 25.vi.1920, 1 male, JSS 19516, R.C. Shannon (CNC); Savary, 29.iv.1916, 1 female, JSS 21329, (MCZ); Waha, 30.v.1924, 1 female, ISS 23778, A.L. Melander (USNM); New Mexico: Las Cruces, Dona Ana, [32.3 N, -106.7666667 W], 1 female, CNCD 26423, F.M. Hull 
(CNC); Sierra Blanca, Lincoln Co., [33.478065 N, -105.552907 W], 2957m, 1026.vi.1979, 1 male, JSS 18307, S. \& J. Peck (CNC); Santa Fe National Forest, San Miguel Co., [35.705996 N, -105.545731 W], 27.v.1981, 1 female, JSS 18346, B.J. Sinclair (CNC); Jemez Springs, Sandoval County, [35.771336 N, -106.690555 W], iv, 1 female, CNCD 26422, (CNC); Utah: Daniels Pass, 2 miles South, Wasatch County, [40.280034 N, -111.223675 W], 2591m, 9.vii.1961, 1 female, CNCD 26421, J.G. Chillcott (CNC); 2 miles West of Mirror Lake, Duschene County, [40.70363 N, 110.928511 W], 23.vi.1968, 1 male, CNCD 26438, B.V. Peterson (CNC); Card Canyon, Cache County, [41.76236 N, -111.663482 W], 13.vi.1937, 1 female, CNCD 26420, A.E. Nye (CNC); Cacha Co., Cowley Canyon, [41.772669 N, -111.60407 W], 1327.v.1989, 1 male, JSS 24612, W.J. Hanson (EMUS); Logan Canyon Summit, Rich County, [41.862735 N, -111.494404 W], 26.vi.-2.vii.1982, JSS 25098, (EMUS); Wasatch-Cache National Forest, Tony Grove Lake, [41.893136 N, -111.641841 W], 1.vii.2010, CNCD 60037-60040, 2 males, JSS 24615, 24616, M.M. Locke (CNC); West Hodges Canyon, Cache County, [41.910305 N, -111.42277 W], 13-20.vi.1980, JSS 25096, (EMUS); Cache Co., Left Hand Fork, Blacksmith Fork Canyon, 24.v.2004, 1 female, JSS 24617, Brammer (EMUS); Wyoming: Lewis Lake, Yellowstone National Park, [44.306126 N, -110.537673 W], 17.vii.1923, 1 female, JSS 19500, A.L. Melander (CNC); Yellowstone Park; Lewis Lake, 17.vii.1923, 1 female, JSS 23774, A.L. Melander (USNM).

\section{Dasysyrphus pinastri (DeGeer)}


Figures: 11C, D, 16B \& 25

Synonyms and References:

Musca pinastri De Geer, 1776: 113, Pl. 7 Fig. 1-7 (original description)

Syrphus lunulatus of authors (nec. Meigen)

Dasysyrphus pinastri (De Geer):

Vockeroth, 1992: 70 (key and redescription)

Doczkal, 1996: 40 (discussion and character chart)

Mutin \& Barkalov, 1999: 398 (key)

Stubbs \& Falk, 2002: 88 (key and discussion)

Reemer, 2002: 14 (key and discussion)

van Veen, 2004: 88 (key)

Barkalov, 2007: 288 (key and redescription)

Bartsch et al., 2009: 199 (key and redescription)

Krpac et al., 2009: 105 (discussion)

Speight, 2011: 71 (discussion)

Diagnosis: Abdominal maculae of tergites $3 \& 4$ slightly arcuate, uniform in thickness and do not cross abdominal margin (Figs 11C, D); very similar in appearance to $D$. pauxillus (Fig. 11A), D. pacificus (Fig. 10D), D. nigricornis (Figs 7A, C) and D. laticaudus (Fig. 7E), sternite 2 with dark macula, dark marking is fasciate in the other species. Male surstylus with posterolateral corner is much less prominent (Fig. 16B) than in other pauxillus group species (Figs 14B, 15A, 15B, 16A). 
Description:

Body Length: $8.4-10.4 \mathrm{~mm}$; Wing Length: $7.8-9.0 \mathrm{~mm}$

Head: Frons dark with light pollenose fascia (in males it runs along ventral edge of where eyes meet, in females it is $1 / 3$ the length between the antennae and ocelli) and dark pile; face light with dark vitta $1 / 4-1 / 3$ the width of the face, not reaching antennal socket, face with light pile; gena dark (sometimes slightly lighter posterioventrally) with light pile and usually some dark pile below eye; occiput dark, covered in light pollen, pile light; scape, pedicel and flagellomere dark, usually uniform in colour, flagellomere sometimes appears slightly lighter.

Thorax: Scutum dark, sometime shiny or metallic, pile light; scutellum light with darker anterolateral edges, dark pile with few light pile along anterior edge; wing cells $r_{1}$ and br usually with bare base (in br above spurious vein only), cell $c$ sometimes with very small bare areas, cup with very small bare areas below vein $\mathrm{CuP}, \mathrm{bm}$ densely microtrichose throughout; haltere light but can be slightly darkened in areas; femora basally dark, apically light, tibiae light, metatibia sometimes with dark band close to apex or darker apical end, tarsi light, meta tarsi with darker anterior side. 
Abdomen: Maculae on tergite 2 large and oval, maculae on tergites $3 \& 4$ are oblique, anterior edge slightly concave and posterior edge straight, never meeting medially, maculae do not reach abdominal margin (Figs $11 \mathrm{C}, \mathrm{D}$ ); sternite 2 with dark macula, sternites $3 \& 4$ light with dark fasciae.

Male Genitalia: Surstylus more or less triangular in shape in lateral view, flattened posteriorly and posterolateral corner is much less prominent than in other pauxillus group species, long pile on dorsal, laterodorsal and posterodorsal sides, row of spines on posteriomedial edge; cercus oval with long pile over entire surface; gonostylus boot-shaped with projection on posterodorsal surface, projecting dorsally, pile on dorsal surface; basiphallus elongate, basally bent at a slightly more than 90 degree angle towards dorsum, apical end curved ventrally and partly membranous dorsally, with spines on posterodorsal side; distiphallus elongate, apical end flared out into horn, which is mostly sclerotized but membranous at apex, with few setulae dorsally, basally it is curved towards dorsum and not greatly enlarged at bend (Fig. 16B).

Intraspecific variation: Gena is dark but in some specimens can be slightly lighter posteroventrally; wing cells $r_{1}$ and br usually with bare base, cell c sometimes with very small bare areas.

Etymology: From the Latin pinus, meaning pine and -aster, a diminutive suffix meaning little. 
Distribution: Greenland and Europe (Fig. 25).

Ecology: Collected from May to July. One specimen was collected from an elevation of $2200 \mathrm{~m}$.

Discussion: Within the Nearctic D. pinastri is only found in Greenland. It is found throughout the Palaearctic from the United Kingdom to Sweden, south to Italy. Due to its similar habitus to other species in the Palaearctic there has been widespread confusion about this species and its concept (Doczkal 1996; Reemer 2002; Speight 2011). The main reason for such confusion is that the syntypes of this species are lost (Thompson \& Pont 1994). They were not in the De Geer collection when it was donated to the Naturhistoriska Riksmuseet (The Swedish Museum of Natural History) in Stockholm, Sweden, in 1834 (Thompson \& Nielsen 1984; Thompson \& Pont 1994). Thompson \& Pont (1994) designated the specimen illustrated by De Geer (1776, Pl. 7 Fig. 1) as the lectotype for pinastri. Since this lectotype has been missing for over 175 years and because this has lead to confusion about the species concept, a neotype is designated here to bring stability of the name pinastri. This designation will create a solid concept for this species, allowing for better reconciliation with closely related species. Characters are consistent with De Geer's (1776) vague description of the adult and with Doczkal's (1996) characters used to distinguish it from pauxillus s.l. and lenensis. De Geer (1776) does not state the locality of his specimens, however, as he was from Sweden and reared these flies 
from larvae it is likely that he collected them close to home. Therefore the Neotype chosen is from Sweden. The Neotype is a male from Holmsund Vb., Sweden, collected on 1.vii.1967 by W.J. Boyes. It is deposited in the CNC and databased with the number Jeff Skevington Specimen 18310. The genitalia have been dissected and a leg was removed to sequence the barcoding region of $\mathrm{COI}$.

Type Material: "Holmsund/ Vb., SWEDEN/ 1.VII.1967/ J.W. Boyes", "Boyes Cytolog./ Coll. \# 615101/ To remain/ in C.N.C.", "J. Skevington/ Specimen \#/ 18310", “Dasysyrphus/ pinastri DeG./ Det. J.R. Vockeroth", "NEOTYPE/ Dasysyrphus/ pinastri/ De Geer/ Det. M.M. Locke"; male; right meso- and metalegs missing; genitalia dissected, in vial on specimen pin; deposited in the Canadian National Collection of Insects, Arachnids and Nematodes (CNC), Ottawa, Ontario, Canada.

Material Examined:

Denmark: North Denmark Region: Bangsbo Skole, North Denmark Region, [57.424683 N, 10.519762 W], 2.vi.1967, 1 male, CNCD 26448, E. Torp (CNC); Syddanmark: Draved Skov, Syddanmark, [55.016651 N, 8.983375 W], 10.v.1966, 1 male, CNCD 26447, 1 female, CNCD 26455, E. Torp (CNC); Greenland: Sondrestom Air Base, [67.010556 N, -50.709167 W], 30.viii.1952, 1 female, CNCD 26459, W.J. Brown (CNC); Italy: Piedmont: Celle de Bellino, Piedmont, [44.579948 N, 7.007859 W], 11-12.vii.1974, intersex, JSS 18309, W. Baker (CNC); Sweden: Vasterbottens Lan: Holmsund, Vasterbottens Lan, [63.705557 N, 20.37888 W], 3.vii.1967, 1 male, JSS 18311, 1 female, CNCD 26453, J.W. Boyes (CNC); Holmsund, Vasterbottens Lan, 
[63.705557 N, 20.37888 W], 1.vii.1967, 1 female, CNCD 26454, J.W. Boyes (CNC); Szklarska Porpba, distr. Telenia Gora Taka, 9.vii.1958, 1 male, CNCD 26449, R. Trojan (CNC); Fellins Skov, 22.v.1967, 1 male, CNCD 26450, E. Torp (CNC); Hytter Kobbel, Sdrj., 24.vi.1967, 1 female, CNCD 26456, E. Torp (CNC); Lillebro, Loso, 25.vi.1966, 1 female, CNCD 26457, E. Torp (CNC); F Zermatt, Rifolalb, 2200m, 23.vi.1959, 1 female, CNCD 26458, F. Keiser (CNC); Switzerland: Jura: Delemont, Jura, [47.365206 N, 7.343584 W], 17-24.v.1969, 1 male, JSS 18308, Herting \& Wood (CNC); United Kingdom: England: Westbury, Bristol, Gloucestershire, [51.502888 N, -2.601311 W], 18.v.1959, 1 male, CNCD 26446, M. Ackland (CNC); Newcastleunder-Lyme, Staffordshire, [53.008764 N, -2.231836 W], 6.vi.1960, 1 female, CNCD 26452, J.R. Vockeroth (CNC); Blea Tarn, Lakes District National Park, Cumbria, [54.429748 N, -3.092892 W], 5.vi.1941, 2 male,s CNCD 26444, 26445, G.E. Shewell $(\mathrm{CNC})$.

\section{Dasysyrphus richardi Locke \& Skevington sp. nov.}

Figures: 9 B, D \& 20

Diagnosis: Similar in appearance to lotus (Figs 9A, C), with maculae on tergites $3 \& 4$ oblique, uniform in thickness, meeting medially and not crossing the abdominal margin (Figs 9 B, D). Dasysyrphus richardi has a black vitta separating the gena from the face (Figs 9B, D) and the wing extensively bare basally, where as lotus is continuously light from face to gena (Fig. 9C) and the wing is more microtrichose. 
Description:

Body Length: $7.2-8.0 \mathrm{~mm}$; Wing Length: $7.2-7.5 \mathrm{~mm}$

Head: Frons dark with light pollenose fascia (in females it is $1 / 2$ the length between the antennae and ocelli and is continuous, sometimes notched medially) and dark pile; face light with dark vitta medially, that is $<1 / 4$ the width of the face, not reaching antennal socket, face with dark pile; gena light with light pile and sometimes interspersed with dark pile, separated from face by dark vitta (Figs 9B, D); occiput dark, covered in light pollen, pile light with some dark pile dorsally; scape and pedicel dark, flagellomere dark, lighter at base.

Thorax: Scutum dark with two faint pollenose vittae medially, extending just past the transverse suture, pile light; scutellum light with darker lateral edges, light pile with some dark pile posteriorly; wing cells br and bm entirely bare, cell $\mathrm{c}$ bare at base and along $S c, r_{1}$ bare at base and cup bare at base and along vein $\mathrm{CuP}$ and posterior edge, $r_{2+3}, r_{4+5}, d m, c u a_{1}$ and anal lobe with bare areas at base; haltere light; femora basally dark, apically light, tibiae light, metatibia sometimes with dark band medially, tarsi light.

Abdomen: Maculae on tergite 2 large, oval to triangular, slightly narrowed on medial end, $1 / 3-<1 / 2$ width of tergite, maculae on tergites $3 \& 4$ are straight and 
oblique, meeting medially, maculae do not reach abdominal margin and are uniform in thickness (Figs. 9B, D); sternites light with dark fasciae of varying thickness.

Male Genitalia: Male unknown.

Intraspecific variation: Maculae on tergite 2 varying slightly in size and shape from $1 / 3-<1 / 2$ width of tergite and oval to slightly more triangular.

Etymology: This species has been named after the systematist Dr. J. Richard Vockeroth, who recognized this as a unique species and labeled the specimens as so, but did not ever describe them.

Distribution: Southwestern USA (NM) (Fig. 20)

Ecology: Two specimens have been collected in June in the southern Rocky Mountains.

Discussion: Known from two female specimens only. They were collected during the same collecting event in New Mexico in 1981.

DNA was not extracted from these specimens as our techniques to extract from 30 year-old specimens need some improvement so that an attempt to extract DNA will have as great a chance as possible of success. With only two known 
specimens in existence this is crucial. Unfortunately with no male specimens, genitalia characters were not available for study. However, because richardi shares such a similar habitus to lotus it is likely that they are within the lotus complex. An effort to find more specimens should be undertaken to locate the male of this species and to have more material made available in order to ascertain the full breadth of its molecular and morphological variation.

Type Material: “New Mexico, Los Alamos/ Bandelier Nat. Mon./ June 1977/ W. Pippen \#D-18", " USNM ENT 00258072", " HOLOTYPE/ Dasysyrphus/ richardi Locke \& Skevington/ Det. M.M. Locke"; female, deposited in the United States National Museum of Natural History, Washington, D.C (USNM).

Material Examined:

Paratype: United States of America: New Mexico: Los Alamos, Bandelier Nat. Mon., [35.783115 N, -106.25619 W], vi.1977, 1 female, USNM ENT 258073, W. Pippin (USNM).

\section{Dasysyrphus venustus (Meigen)}

Figures: 8 A, B, 17B, 26

Synonyms and References:

Scaeva arcuata Fallén: 1817: 42 (original description) 
Dusek \& Laska, 1969: 171 (name suppression proposal)

International Commission on Zoological Nomenclature 1972, 1972: 9 (name suppression)

Syrphus venustus Meigen, 1822: 299 (original description)

Syrphus lunulatus Meigen 1822: 299 (original description)

Syrphus reflectipennis Curran, 1921: 157 (original description)

Metasyrphus reflectipennis (Curran):

Fluke, 1933: 109 (key, redescription and transfer to Metasyrphus)

Metasyrphus venustus (Meigen):

Fluke, 1933: 112 (key, redescription and transfer to Metasyrphus)

Dasysyrphus lunulatus (Meigen):

Enderlein, 1938: 208 (transfer to Dasysyrphus)

Stone et al., 1965: 564 (catalogue and transfer back to Dasysyrphus)

Goeldlin de Tiefnau, 1974: 208 (lectotype designation)

Syrphella lunulatus (Meigen):

Goffe, 1944: 129 (transfer to Syrphella)

Syrphella venustus (Meigen):

Goffe, 1944: 129 (transfer to Syrphella)

Dasysyrphus arcuatus (Fallén):

Stone et al., 1965: 563 (catalogue and transfer to Dasysyrphus)

Peck, 1974: 128 (key)

Dasysyrphus reflectipennis (Curran):

Stone et al., 1965: 564 (catalogue and transfer to Dasysyrphus) 
Dasysyrphus venustus (Meigen):

Dusek \& Laska, 1967: 354 (transfer to Dasysyrphus?)

Goeldlin de Tiefnau, 1974: 208 (lectotype designation)

Vockeroth, 1986: 203 (discussion)

Peck, 1988: 16 (catalogue)

Vockeroth, 1992: 70 (key and redescription)

Mutin \& Barkalov, 1999: 397 (key)

Stubbs \& Falk, 2002: 88 (key and discussion)

van Veen, 2004: 87 (key)

Barkalov, 2007: 294 (key and redescription)

Bartsch et al., 2009: 202 (key and redescription)

Speight, 2011: 72 (discussion)

Diagnosis: Abdominal maculae cross abdominal margin; maculae on tergite 2 large

$(>1 / 3$ width of tergite) oval-shaped; maculae on tergites $3 \& 4$ are transverse, constricted slightly medially, with medial edge swollen slightly (Figs 8A, B). Similar to D. limatus however maculae on tergite 2 are always small in D. limatus (Fig. 8C), less than $1 / 4$ the width of the tergite, and are much larger in $D$. venustus, greater than $1 / 3$ the width of the tergite. Also often confused with species in D. intrudens complex, however, species in this complex have maculae that are arcuate, lunulate and/or greatly constricted medially.

Description: 
Body Length: $7.9-11.2 \mathrm{~mm}$; Wing Length: $7.4-10.4 \mathrm{~mm}$

Head: Frons dark with light pollenose fascia (in males it runs along ventral edge of where eyes meet, in females it is $1 / 3-1 / 2$ the length between the antennae and ocelli and widely separated medially) and dark pile; face light with dark vitta $1 / 4-1 / 3$ the width of the face, usually reaching antennal socket, face with light to dark pile or combination of light and dark pile; gena dark with dark to light pile or a combination of light and dark pile; occiput dark, covered in light pollen, pile light; scape and pedicel light, flagellomere usually light basally and darker apically, sometimes mostly dark.

Thorax: Scutum dark, sometime shiny or metallic, pile light; scutellum light with 'darker anterolateral edges, light pile some with few dark pile along posterior edge; wings can range from being entirely densely microtrichose to having wing cells $r_{1}$, br and bm bare base (in br above spurious vein only) and cell cup with small bare areas below vein CuP; haltere light; femora basally dark, apically light, tibiae light, metatibia with dark band close to apex, tarsi light, meta tarsi darker.

Abdomen: Maculae on tergite 2 large and oval with anterolateral edge extending to edge of abdomen, maculae on tergites $3 \& 4$ are transverse, constricted slightly medially, with medial edge swollen slightly, almost never meeting medially, . 
maculae reach abdominal margin (Figs 8A, B); sternite 2 either all light or with dark fascia (usually distinct, sometimes faint), sternites $3 \& 4$ light with dark fasciae.

Male Genitalia: Surstylus more or less triangular in shape in lateral view, flattened anteroposteriorly with long pile on posterior side and spines on anteroventral side, similar to D. intrudens complex (Fig. 17A) and D. limatus (Fig. 13B); cercus oval with long pile over entire surface; gonostylus is pointed ventrally, dorsally flattened at base then it expands dorsally at apex, oblique ridge near apex, with ends of ridge projecting into points; pile on dorsal surface; basiphallus is basally bent at about a 90 degree angle towards dorsum, apical end enlarged and curved ventrally, fully sclerotized all the way around with no pile or spines; distiphallus long with no enlarged area basally, apical end flared out into horn with the dorsal side flattened slightly, which is mostly sclerotized but membranous at apex with setulae dorsally (Fig. 17B).

Intraspecific variation: The abdominal maculae of the male are sometimes more arcuate and with a more swollen medial edge of maculae. Wings cells $r_{1}, b r, b m$, and cup range from being entirely microtrichose to having bare areas.

Etymology: From the Latin venustus, meaning charming, lovely, beautiful, graceful.

Distribution: Canada (AB, BC, MB, NT, NS, ON, QC, YT) and US (AK, ID, ME, MI, NH, NM, NC, PA, UT, VT, VA, WV), also widespread over Europe and Asia (Fig. 26). 
Ecology: Collected from May - August, but more frequently in May and June. Collected at elevations from 260-2957m. Habitats listed on labels include "bog", "fell-field tundra", "Mh/Be forest" (mixed hardwood/beech), "pine forest", "boreal mixed wood forest", "black spruce forest (25\%) with moss", "Picea-Betula forest". Specimens have been collected from hilltops.

Discussion: The species concept for $D$. venustus has been uncertain for quite some time now, especially in the Nearctic. Fluke's (1933) concept was much like that of this one, distinguishing venustus as having markings that are more straight than specimens in the intrudens complex, which is treated by Fluke as laticaudatus, amalopis (in part) and osburni. Vockeroth (1986) synonymized the intrudens complex under venustus as he believed it was simply a variable species. While abdominal maculae vary greatly, many of the other characters are similar, including the male genitalia.

Dasysyrphus osburni, intrudens, disgregus and laticaudatus were all considered synonyms of venustus by Vockeroth (1992), however these four names fall within the intrudens complex.

The only specimen of $D$. reflectipennis is that of the holotype (located at the $\mathrm{CNC}$ ). It is a female with its abdomen broken and glued to a point below the specimen. It was treated as a unique species by Fluke (1933) and by Vockeroth 
(1986). However, at the time, Vockeroth believed that reflectipennis was a specimen of venustus with aberrant markings. He stated that he was not ready to treat it as a synonym until other specimens were found. Other specimens were not found, but in 1992 he synonymized reflectipennis with venustus. The abdominal maculae of tergite 3 are like that of venustus, however they narrowly meet medially. I have seen no other specimens where the maculae meet, but since the specimen of reflectipennis looks like that of venustus in every other way, it is logical to conclude that Vockeroth was correct in saying that the specimen of reflectipennis is actually a specimen of venustus with aberrant markings.

There are five specimens with identical morphology to venustus that differ from it in their COI sequences (CNCD 5458, 30889, 30890, 30902, JSS 20982; Fig. 4). There is no difference in geographic range either. It is possible that this is a cryptic species but until more specimens are available to provide more precise geographical and ecological evidence and/or morphological and DNA characters they are retained under the name venustus.

Type Material: From Germany, deposited in the Muséum National d'Histoire Naturelle (The National Museum of Natural History), Paris, France (Pape \& Thompson, 2010). (not examined; requested but not obtained)

Material Examined: 
Canada: Alberta: Pigeon Lake, Itaska Audubon Reserve, [53 N, -114 W], 18.v.2008, 1 male, JSS 21073, F.A.H. Sperling (FAHS); Pigeon Lake, Itaska Audubon Reserve, [53 N, -114 W], 18.v.2008, 1 female, JSS 21074, F.A.H. Sperling (FAHS); Drayton, [53.220865 N, -114.978236 W], 29.vi.1988, 1 female, UASM\# 149016, E.H. Strickland (UASM); Edmonton, Division No.11, [53.540941 N, -113.493698 W], 5.vi.1948, 1 female, CNCD 14537, J.R. Vockeroth (CNC); Edmonton, Division No.11, [53.540941 N, -113.493698 W], 20.v.1923, 1 female, CNCD 14538, E.H. Strickland (CNC); Edmonton, Division No.11, [53.540941 N, -113.493698 W], 12.v.1924, 1 female, JSS 19540-19545, 19551-19554, 19561-19564, 0. Bryant (CNC); Edmonton, Division No.11, [53.540941 N, -113.493698 W], 13.v.1924, 1 female, JSS 19559, 19560, 19565, O. Bryant (CNC); Edmonton, Division No.11, [53.540941 N, 113.493698 W], 16.v.1924, 1 female, JSS 19558, O. Bryant (CNC); Elk Island National Park, [53.604578 N, -112.859291 W], 19.v.1981, 1 female, CNCD 14540, J.E. O'Hara (CNC); Bilby, Division No. 13, [53.698357 N, -114.10198 W], 1.vi.1924, 1 female, JSS 19546, 0. Bryant (CNC); British Columbia: Robson, [49.333015 N, 117.692871 W], 14.v.1954, 1 male, JSS 20034, H.R. Foxlee (UBCZ); Robson, [49.333015 N, -117.692871 W], 17.v.1954, 1 male, JSS 20025, H.R. Foxlee (UBCZ); Robson, [49.333015 N, -117.692871 W], 19.v.1954, JSS 20127, H.R. Foxlee (UBCZ); Robson, [49.333015 N, -117.692871 W], 26.v.1954, 1 male, JSS 19999, H.R. Foxlee (UBCZ);

Robson, [49.333015 N, -117.692871 W], 20.v.1955, 1 male, JSS 19998, H.R. Foxlee (UBCZ); Robson, [49.333015 N, -117.692871 W], 7.v.1956, 1 female, ISS 20015, H.R. Foxlee (UBCZ); Robson, [49.333015 N, -117.692871 W], 8.v.1956, 1 male, JSS 
20019, H.R. Foxlee (UBCZ); Robson, [49.333015 N, -117.692871 W], 14.v.1956, 3 males, JSS 20023, 20164, H.R. Foxlee (UBCZ); Robson, [49.333015 N, -117.692871 W], 15.v.1956, 1 male, JSS 20132, 1 female, JSS 20130, H.R. Foxlee (UBCZ); Robson, [49.333015 N, -117.692871 W], 6.v.1957, 1 male, ISS 20012, H.R. Foxlee (UBCZ); Robson, [49.333015 N, -117.692871 W], 11.v.1957, 1 female, JSS 20002, H.R. Foxlee (UBCZ); Robson, [49.333015 N, -117.692871 W], 21.v.1957, 1 female, JSS 20095, H.R. Foxlee (UBCZ); Robson, [49.333015 N, -117.692871 W], 7.vi.1965, 1 female, JSS 20122, H.R. Foxlee (UBCZ); Robson, [49.333015 N, -117.692871 W], 25.v.1965, 1 female, JSS 20092, H.R. Foxlee (UBCZ); Robson, Central Kootenay L.D., [49.342029 N, -117.697831 W], 11.v.1948, 1 female, CNCD 14242, H.R. Foxlee (CNC); Robson, Central Kootenay L.D., [49.342029 N, -117.697831 W], 2.vi.1948, 1 female, CNCD 14303, H.R. Foxlee (CNC); Robson, Central Kootenay L.D., [49.342029 N, 117.697831 W], 14.v.1947, 1 male, CNCD 14510, H.R. Foxlee (CNC); Miracle Beach, Near Oyster Bay, [49.854574 N, -125.098409 W], 13.vi.1955, 1 female, CNCD 14314, J.R. McGillis (CNC); Salmon Arm, [50.703923 N, -119.273924 W], 2.v.1930, 1 female, JSS 20159, Hugh B. Leech (UBCZ); 32 miles South West of Terrace, [54.194076 N, 129.140296 W], 30.5m, 11.vi.1960, 2 females, CNCD 14217, 14341, B. Heming (CNC); 32 miles South West of Terrace, [54.194076 N, -129.140296 W], 8.vi.1960, 1 male, CNCD 14509, G.E. Shewell (CNC); Spring Creek, Terrace, [54.539511 N, 128.618192 W], 1.vi.1960, 1 female, CNCD 14231, C.H. Mann (CNC); Kitsumkalum Lake, 20 Miles North of Terrace, [54.762473 N, -128.770968 W], 16.vi.1960, 1 female, CNCD 14227, J,G. Chillcott (CNC); Kispiox, [55.348319 N, -127.687346 W], 24.v.1958, 1 male, JSS 20152, J. Foster (UBCZ); Atlin, [59.5775 N, -133.69236 W], 
670.56m, 20.vi.1955, 1 female, CNCD 14223, B.A. Gibbard (CNC); Atlin, [59.5775 N, 133.69236 W], 670.56m, 9.vi.1955, 2 females, CNCD 14251, 14252, B.A. Gibbard (CNC); Atlin, [59.5775 N, -133.69236 W], 670.56m, 6.vii.1955, 1 female, CNCD 14284, B.A. Gibbard (CNC); Atlin, [59.5775 N, -133.69236 W], 670.56m, 7.vii.1955, 1 female, CNCD 14289, H.J. Huckel (CNC); Atlin, [59.5775 N, -133.69236 W], 670.56m, 20.vi.1955, 1 male, CNCD 14360, H. Huckel (CNC); Blanchard R. Haines Rd., [11.vi.1979, 1 female, JSS 20146, G.J. Spencer (UBCZ); Kiskatinaw Public Campground, Alaska Highway DC-20 (31.4km), [26.vi.1978, 1 female, JSS 23719, Paul H. Arnaud, Jr. (CAS); Manitoba: Aweme, [49.708529 N, -99.602758 W], 19.v.1926, 1 female, CNCD 14550, R.D. Bird (CNC); Aweme, [49.708529 N, 99.602758 W], 20.vi.1925, 1 female, CNCD 14551, R.M. White (CNC); Aweme, [49.708529 N, -99.602758 W], 27.v.1925, 1 female, CNCD 14554, R.D. Bird (CNC); Aweme, [49.708529 N, -99.602758 W], 1.vii.1966, 1 female, CNCD 14555, R.D. Bird (CNC); 5 miles South West of Shilo, [49.763964 N, -99.717403 W], 28.v.1958, 1 female, CNCD 14552, J.F. McAlpine (CNC); 5 miles South West of Shilo, [49.763964 N, -99.717403 W], 5.vi.1958, 1 female, CNCD 14553, C.D.F. Miller (CNC); Erickson, [50.498283 N, -99.9078 W], 17.vi.1983, 1 female, JSS 20195, D.H. Pengelly (JBWM); Churchill Area, Burn site N[orth] of Twin lakes, [58.61861111 N, -93.82888889 W], 4.vii.2007, 1 female, CNCD 9495, J. Skevington (CNC); Churchill Area, Burn site $\mathrm{N}$ [orth] of Twin lakes, [58.61861111 N, $-93.82888889 \mathrm{~W}], 5 . v i i .2007,1$ female, CNCD 9582, J. Skevington (CNC); Churchill, Bus Stop, [7.viii.2007, 1 female, JSS 24644, P. Kevan (CNC); New Brunswick: Chamcook, Charlotte Co., [45.126407 N, 67.071693 W], 28.vi.1965, 3 females, CNCD 14817-14819, G.E. Shewell (CNC); 
Doaktown, Northumberland Co., [46.556585 N, -66.126203 W], 6.vii.1971, 1 female, CNCD 14820, B.V. Peterson (CNC); Kouchibouguac National Park, Kent Co., [46.819201 N, -64.96788 W], 19.vi.1978, 2 females, CNCD 14815, 14816, S.J. Miller (CNC); Snow Cap Park, H[igh]w[a]y 17, [5.vii.1971, 1 female, CNCD 14821, B.V. Peterson (CNC); Northwest Territories: Jean-Marie Creek, 42 Miles South West of Fort Simpson, [61.372555 N, -122.224182 W], 13.vi.1972, 1 female, CNCD 14047, B.V. Peterson (CNC); Coppermine, [62.562732 N, -115.092764 W], 30.vi.1951, 1 female, CNCD 14058, S.D. Hicks (CNC); Wrigley, Mackenzie, [63.209367 N, 123.34594 W], 15.vi.1969, 1 female, CNCD 14056, G.E. Shewell (CNC); Nova Scotia: Kings County, [45.017428 N, -64.610757 W], 23.vi.1983, 1 female, CNCD 14778, J.R. Vockeroth (CNC); Kings County, [45.017428 N, -64.610757 W], 22.vi.1983, 1 female, CNCD 14779, J.R. Vockeroth (CNC); Truro, Colchester Co., [45.364959 N, -63.279846 W], 7.vii.1919, 1 male, CNCD 14769, (CNC); Baddeck, Victoria Co., Cape Breton Island, [46.10001 N, -60.753507 W], 15.vi.1931, 1 female, CNCD 14780, G. Fairchild (CNC); Cape Breton Highlands National Park, French Lake, Inverness Co., [46.728452 N, -60.86483 W], 24-30.vi.1984, 2 females, CNCD 14774, 14775, H.J. Teskey (CNC); Cape Breton Highlands National Park, North Mtn. Bog, Inverness Co., [46.805111 N, $-60.688224 \mathrm{~W}], 400 \mathrm{~m}, 7 . v i .1984,2$ males, CNCD 14759, 14760, B.E. Cooper (CNC); Cape Breton Highlands National Park, Mackenzie Mountain, Inverness Co., [46.773977 N, -60.818772 W], 400m, 9.vi.1984, 1 male, CNCD 14762, B.E. Cooper (CNC); Cape Breton Highlands National Park, Pleasant Bay, Inverness Co., [46.822961 N, -60.799065 W], 6.vi.1984, 1 male, CNCD 14765, B.E. Cooper (CNC); Cape Breton Highlands National Park, Pleasant Bay, Inverness Co., 
[46.822961 N, -60.799065 W], 11.vi.1984, 1 male, CNCD 14763, B.E. Cooper (CNC); Cape Breton Highlands National Park, Pleasant Bay, Inverness Co., [46.822961 N, 60.799065 W], 12.vi.1984, 3 males, CNCD 14757, 14758, 14766, 2 females, CNCD 14770, 14771, B.E. Cooper (CNC); Cape Breton Highlands National Park, Pleasant Bay, Inverness Co., [46.822961 N, -60.799065 W], 25-29.vi.1984, 1 female, CNCD 14776, H.J. Teskey (CNC); Dingwall, Victoria Co., [46.896638 N, -60.469402 W], 23.vi.1983, 1 female, CNCD 14777, J.R. Vockeroth (CNC); Ontario: Crawford, [44.253882 N, -80.929867 W], 8-17.vii.1998, ROMEnt Spec. No. 74031, A. Sugar (ROME); Crawford twp., [44.261716 N, -80.932034 W], 7-16.vi.1998, 4 females, ROMEnt Spec. No. 57872, 57887-57889, Alissa Sugar (ROME); Cranberry Lake, Runtz property, [44.44111111 N, -76.29055556 W], 18-24.v.2007, 2 females, CNCD 30889, 30890, J. Skevington, H. Douglas, D. Runtz (CNC); Chaffeys Lock, Leeds County, [44.579098 N, -76.319816 W], 28.v.1972, 1 female, CNCD 14593, P. Ward (CNC); Orillia, Simcoe Co., [44.609505 N, -79.42068 W], 7.vi.1925, 1 female, CNCD 14594, C.H. Curran (CNC); Orillia, Simcoe Co., [44.609505 N, -79.42068 W], 30.v.1921, 1 female, CNCD 14595, C.H. Curran (CNC); Orillia, Simcoe Co., [44.609505 N, -79.42068 W], 26.v.1913, 1 female, CNCD 15386, C.H. Curran (CNC); Midland, Simcoe Co., [44.752113 N, -79.887253 W], 26.v.1959, 1 female, CNCD 14600, J.G. Chillcott (CNC); Griffith, 7 miles East, [45.243279 N, -77.031193 W], 22.v.1982, 1 male, CNCD 14567, B.E. Cooper (CNC); Stittsville, Ottawa Division, [45.263479 N, 75.925163 W], 24.vi.1963, 1 female, CNCD 14598, W.R.M. Mason (CNC); Nepean, 24 Gervin Street, [45.31694444 N, -75.72 W], 28.v.2006 4.vi.2006, 1 female, CNCD 30888, J.E. O'Hara (CNC); Mer Bleu, 5 Miles East of Ottawa, Ottawa Division, 
[45.40331 N, -75.5083 W], 31.v.1923, 1 female, CNCD 14579, C.H. Curran (CNC); Mer Bleu, 5 Miles East of Ottawa, Ottawa Division, [45.40331 N, -75.5083 W], 5.vi.1966, 2 females, CNCD 14585, 14586, D.D. Munroe (CNC); Mer Bleu, 5 Miles East of Ottawa, Ottawa Division, [45.40331 N, -75.5083 W], 13.vi.1966, 1 female, CNCD 14587, D.D. Munroe (CNC); Mer Bleu, 5 Miles East of Ottawa, Ottawa Division, [45.40331 N, -75.5083 W], 15.vi.1966, 1 female, CNCD 14588, D.D. Munroe (CNC); Mer Bleu, 5 Miles East of Ottawa, Ottawa Division, [45.40331 N, -75.5083 W], 17.vi.1966, 1 female, CNCD 14589, D.D. Munroe (CNC); Mer Bleu, 5 Miles East of Ottawa, Ottawa Division, [45.40331 N, -75.5083 W], 19.vi.1966, 2 females, CNCD 14590, 14591, D.D. Munroe (CNC); Mer Bleu, 5 Miles East of Ottawa, Ottawa Division, [45.40331 N, -75.5083 W], 1.vii.1966, 1 female, CNCD 14592, D.D. Munroe (CNC); Ottawa, Ottawa Division, [45.411604 N, -75.688669 W], 29.v.1925, 1 female, CNCD 14571, C.H. Curran (CNC); By Florence L. Algonquin P.P. [Florence Lake, Algonquin Provincial Park], [45.44371 N, -78.49012 W], 13.v.2007 29.v.2007, 1 male, JSS 21385, E. Proctor (CNC); Waubamick, Parry Sound District, [45.446658 N, -80.017278 W], 14.vi.1915, 2 females, JSS 19533, 19534, E.S. Parish (CNC); By Cecil Lake, Algonquin Provincial Park, [45.4511 N, -78.4956 W], 17.v.2007 29.v.2007, 1 male, JSS 21371, E. Proctor (CNC); 2 km E of Pondweed L. [Lake], Algonquin Provincial Park, [45.4654 N, -78.4298 W], 16-28.v.2007, 2 males, JSS 21394, 21397, E. Proctor (CNC); Dirleton, Ottawa Division, [45.494944 N, -76.142874 W], 29.iv.1968, 1 female, CNCD 14599, J.E.H. Martin (CNC); Algonquin Park, [46 N, 78.08333333 W], 10.vii.2003 17.vii.2003, 1 female, ROMEnt Spec. No. 98534, Mark Vanderwel (ROME); Algonquin Park, [45.86666667 N, -78.38333333 W], 12- 
19.vi.2003, 1 female, ROMEnt Spec. No. 98532, Mark Vanderwel (ROME); Algonquin Park, [45.76666667 N, -78.08333333 W], 13.-20.vi.2003, 7 females, ROMEnt Spec. No. 98522-98525, 98535, 98536, 98540, Mark Vanderwel (ROME); Algonquin Park, [45.86666667 N, -78.38333333 W], 17-24.vi.2003, 1 female, ROMEnt Spec. No. 98541, Mark Vanderwel (ROME); Algonquin Park, [45.86666667 N, -78.38333333 W], 18-25.vi.2003, 3 females, ROMEnt Spec. No. 98526, 98527, 98528, 98533, Mark Vanderwel (ROME); Algonquin Park, [45.7 N, -78.08333333 W], 20-27.vi.2003, 2 females, ROMEnt Spec. No. 98530, 98531, Mark Vanderwel (ROME); Atikokan, 14 miles East on Hwy 11, [48.723518 N, -91.313476 W], 3.vii.1978 4.vii.1948, 1 female, CNCD 14582, H.J. Teskey (CNC); Iroquois Falls, [48.761811 N, -80.685262 W], 20.vi.1987, 1 female, JSS 20773, J.R. Vockeroth (CNC); LowBush, Lake Abitibi, [48.91608 N, -80.139852 W], 6.vi.1925, 1 male, BOC 34653, N.K. Bigelow (ROME); LowBush, Lake Abitibi, [48.91608 N, -80.139852 W], 7.vi.1925, 2 females, ROMEnt Spec. No. 115126, 115127, N.K. Bigelow (ROME); Low Bush, Lake Abitibi, Cochrane District, [48.91608 N, -80.139852 W], 9.vi.1925, 1 female, CNCD 14572, N.K. Bigelow (CNC); LowBush, Lake Abitibi, [48.91608 N, -80.139852 W], 13.vi.1925, 2 females, ROMEnt Spec. No. 115128, 115130, N.K. Bigelow (ROME); Low Bush, Lake Abitibi, Cochrane District, [48.91608 N, -80.139852 W], 15.vi.1925, 1 female, CNCD 14573, N.K. Bigelow (CNC); Low Bush, Lake Abitibi, Cochrane District, [48.91608 N, -80.139852 W], 16.vi.1925, 2 females, CNCD 14574-14576,N.K. Bigelow (CNC); LowBush, Lake Abitibi, [48.91608 N, -80.139852 W], 16.vi.1925, 1 female, ROMEnt Spec. No. 115131, N.K. Bigelow (ROME); LowBush, Lake Abitibi, [48.91608 N, 80.139852 W], 17.vi.1925, 1 female, ROMEnt Spec. No. 115132, N.K. Bigelow 
(ROME); LowBush, Lake Abitibi, [48.91608 N, -80.139852 W], 18.vii.1925, 1 female, ROMEnt Spec. No. 115129, N.K. Bigelow (ROME); LowBush, Lake Abitibi, [48.91608 N, -80.139852 W], 23.vi.1925, 1 female, ROMEnt Spec. No. 115133, N.K. Bigelow (ROME); LowBush, Lake Abitibi, [48.91608 N, -80.139852 W], 1.vii.1925, 1 female, UASM\# 149015, N.K. Bigelow ( UASM ); LowBush, Lake Abitibi, [48.91608 N, 80.139852 W], 1.vii.1925, 4 females, BOC 34649-34652, N.K. Bigelow (ROME); Low Bush, Lake Abitibi, [48.91608 N, -80.139852 W], 1.vii.1925, 1 female, JSS 21349, N.K. Bigelow (MCZ); Low Bush, Lake Abitibi, Cochrane District, [48.91608 N, 80.139852 W], 14.vii.1925, 1 female, CNCD 14578, N.K. Bigelow (CNC); Low Bush, Lake Abitíbi, [48.91608 N, -80.139852 W], 14.vii.1925, 1 female, JSS 21350, N.K. Bigelow (MCZ); LowBush, Lake Abitibi, [48.91608 N, -80.139852 W], 14.vii.1925, 1 female, BOC 34654, N.K. Bigelow (ROME); LowBush, Lake Abitibi, [48.91608 N, 80.139852 W], 18.vii.1925, 3 females, BOC 34655-34657, N.K. Bigelow (ROME); Macdiarnid, Lake Nipigon, [49.438216 N, -88.12695 W], 7.vi.1921, 1 female, BOC 34659, N.K. Bigelow (ROME); Macdiarnid, Lake Nipigon, [49.438216 N, -88.12695 W], 24.vi.1923, 1 female, BOC 34660, N.K. Bigelow (ROME); Quebec: Covey Hill, Le Haut-Saint-Laurent, [45.019681 N, -73.745746 W], 20.vi.1924, 1 female, CNCD 14755, G.S. Walley (CNC); Aylmer, [45.400224 N, -75.817137 W], 28.v.1923, 1 male, CNCD 14653, C.H. Curran (CNC); Ste. Anne de Bellevue, [45.406417 N, -73.950944 W], 12.v.1965, 1 female, LEM 8806, W. Boyle (LEMQ); Ste. Anne de Bellevue, [45.406417 N, -73.950944 W], 13.v.1965, 1 female, LEM 8807, G. Jamieson (LEMQ); Ste. Anne de Bellevue, [45.406417 N, -73.950944 W], 1.vi.1966, 1 female, LEM 8804, Wayne Boyle (LEMQ); Ste. Anne de Bellevue, [45.406417 N, -73.950944 W], 
15.v.1967, 1 male, LEM 8805, A. Campbell (LEMQ); Abbotsford, [45.437637 N, 72.887923 W], vii.1935, 1 female, CNCD 14711, G.E. Shewell (CNC); Abbotsford, [45.437637 N, -72.887923 W], 25.vi.1937, 1 female, CNCD 14725, G. Shewell (CNC); Abbotsford, [45.437637 N, -72.887923 W], 14.vi.1972, 1 female, CNCD 14689, D.M. Wood (CNC); [Vaudreuil Co., Summit of] Mount Rigaud, [45.46638889 N, 74.32638889 W], 19.v.2004, 1 male, CNCD 68, J. Skevington, L. Bartels, E. St. Louis, J. King (CNC); Summit of M[oun]t Rigaud, [45.46638889 N, 74 W], 24.v.2007, CNCD $8656,8658,8675$, J. Skevington, A. Bailie, R. Farmahin, A. Kealey, S. Kelso, J. King (CNC); Summit of King Mountain, Old Chelsea, Les Collines-des-L'Outaouais, [45.498058 N, -75.864116 W], 351m, 14.vi.1963, 1 male, CNCD 14680, 2 females, CNCD 14737, 14738, J.R. Vockeroth (CNC); Summit of King Mountain, Old Chelsea, Les Collines-des-L'Outaouais, [45.498058 N, -75.864116 W],351m, 21.vi.1966, 1 male, CNCD 14651, 1 female, CNCD 14686, J.R. Vockeroth (CNC); Summit of King Mountain, Old Chelsea, Les Collines-des-L'Outaouais, [45.498058 N, -75.864116 W], 351m, 13.vi.1970, 6 males, CNCD 14606, 14608-14612, J.R. Vockeroth (CNC); Summit of King Mountain, Old Chelsea, Les Collines-des-L'Outaouais, [45.498058 N, -75.864116 W], 9.vi.1971, 1 male, CNCD 14681, D.M. Wood (CNC); Summit of King Mountain, Old Chelsea, Les Collines-des-L'Outaouais, [45.498058 N, -75.864116 W], 351m, 11.vi.1971, 2 males, CNCD 14654, 14679, 1 female, CNCD 14736, J.R. Vockeroth (CNC); Summit of King Mountain, Old Chelsea, Les Collines-desL'Outaouais, [45.498058 N, -75.864116 W], 351m, 16.vi.1971, 1 male, CNCD 14671, I.R. Vockeroth (CNC); Summit of King Mountain, 0ld Che!sea, Les Collines-desL'Outaouais, [45.498058 N, -75.864116 W], 351m, 12.vi.1980, 1 female, CNCD 
14693, J.R. Vockeroth (CNC); Champlain Lookout, Gatineau Park, Les Collines-del'Outaouais, [45.508314 N, $-75.913273 \mathrm{~W}], 335 \mathrm{~m}, 1 . v i .1965,1$ male, CNCD 14672, J.R. Vockeroth (CNC); Montreal Area, Communaute-Urbaine-de-Montreal, [45.526155 N, -73.702807 W], 6.vi.1964, 2 females, CNCD 14692, 14694, J.W. Boyes (CNC); Mont Saint-Hilaire, La Vallee-du-Richelieu, [45.552948 N, -73.155276 W], 411m, 11.vi.1967, 1 male, CNCD 14675, J.W. Boyes (CNC); Lac-Megantic, Le Granit, [45.57781 N, -70.884105 W], 18.vi.1923, 1 male, CNCD 14646, C.H. Curran (CNC); Lac-Megantic, Le Granit, [45.57781 N, -70.884105 W], 20.vi.1923, 1 female, CNCD 14753, C.H. Curran (CNC); Lac-Megantic, Le Granit, [45.57781 N, -70.884105 W], 30.v.1971, 1 male, CNCD 14667, D.M. Wood (CNC); Duncan Lake, near Rupert, [45.68138889 N, -76.05027778 W], 21.vi.1969, 1 female, CNCD 14745, J.F. McAlpine (CNC); Cté Gatineau Masham Duncan Lake, [45.68138889 N, -76.05027778 W], 260m, 19.v.1987, 1 male, JSS 20767, D.M. Wood (CNC); Lac Cloutier, [46.184459 N, 73.64769 W], 30.v.1987 7.vii.1987, 1 female, LEM 13620, (LEMQ); Schefferville, Iron Arm Fern, [54.805276 N, -66.805274 W], 28.vi.1990, 1 female, LEM 13928, P. McElligott (LEMQ); Schefferville, Iron Arm Fern, [54.805276 N, -66.805274 W], 34.vii.1990, 1 female, LEM 13459, P. McElligott (LEMQ); Great Whale River, [55.116816 N, -76.405554 W], 7.vii.1949, 1 female, CNCD 14718, J.R. Vockeroth (CNC); Great Whale River, [55.116816 N, -76.405554 W], 12.vii.1949, 3 females, CNCD 14715-14717, J.R. Vockeroth (CNC); Great Whale River, [55.116816 N, 76.405554 W], 24.vii.1949, 1 female, CNCD 14724, J.R. Vockeroth (CNC); Great Whale River, [55.116816 N, -76.405554 W], 25.vii.1949, 1 female, CNCD 14714, J.R. Vockeroth (CNC); Great Whale River, [55.116816 N, -76.405554 W], 31.vii.1949, 1 
female, CNCD 14719, J.R. Vockeroth (CNC); Indian House Lake, [56.328482 N, 64.720845 W], 8.vii.1954, 1 female, CNCD 14721, W.R. Richards (CNC); Indian House Lake, [56.328482 N, -64.720845 W], 10.vii.1954, 1 male, CNCD 14683, W.R. Richards (CNC); Indian House Lake, [56.328482 N, -64.720845 W], 17.vii.1954, 1 female, CNCD 14722, W.R. Richards (CNC); Kuujjuaq (Fort Chimo), [58.100076 N, 68.406179 W], 29.v.1948, 1 male, CNCD 14684, H.N. Smith (CNC); Kuujjuaq (Fort Chimo), [58.100076 N, -68.406179 W], 14.vii.1948, 1 female, CNCD 14712, R.H. MacLeod (CNC); Kuujjuaq (Fort Chimo), [58.100076 N, -68.406179 W], 28.vii.1948, 1 female, CNCD 14713, R.H. MacLeod (CNC); Macd. College, [21.v.1959, 1 male, LEM 8815, (LEMQ); Great River, [29.vi.1990 2.vii.1990, 1 female, LEM 13674, G. Pelletier (LEMQ); Saskatchewan: Great Deer, [52.580321 N, -107.056629 W], 21.v.1949, 1 female, CNCD 14547, J.R. Vockeroth (CNC); Nipawin, Division No.14, [53.365608 N, 104.011519 W], 2.vi.1948, 1 female, CNCD 14548, J.R. Vockeroth (CNC); Yukon Territory: Otter Lake, [62.486817 N, -130.4166667 W], 1219m, 17.vii.1960, 1 female, CNCD 13966, J.E.H. Martin (CNC); Sheldon Lake, [62.616763 N, -131.266603 W], 1067m, 3.vii.1960, 1 female, CNCD 13967, J.E.H. Martin (CNC); Sheldon Lake, [62.616763 N, -131.266603 W], 1067m, 3.vii.1960, 1 female, CNCD 13976, E.W. Rockburne (CNC); Sheldon Lake, [62.616763 N, -131.266603 W], 1067m, 7.vii.1960, 1 female, CNCD 13968, J.E.H. Martin (CNC); Sheldon Lake, [62.616763 N, 131.266603 W], 1067m, 7.vii.1960, 2 females, CNCD 13974, 13975, E.W. Rockburne (CNC); La Force Lake, [62.683333 N, -132.333333 W], 1006m, 28.vi.1960, 1 female, CNCD 13955, E.W. Rockburne (CNC); La Force Lake, [62.683333 N, -132.333333 W], 1006m, 7.vii.1960, 1 female, CNCD 13949, J.E.H. Martin (CNC); Mile 40, Dempster 
Highway, [64.448494 N, -138.223783 W], 1-6.vii.1973, 1 male, CNCD 14029, G. \& D.M. Wood (CNC); Engineer Cr., km 152 Dempster Hwy, [65.070828 N, -138.2399 W], 30.vi.1982, 1 female, JSS 20140, S.G. Cannings, L. Vasington, R.A. Moore (UBCZ); km 416 Dempster Highway, [66.644581 N, -136.324471 W], 750m, 22-28.vi.1980, 1 female, CNCD 14005, Wood \& Lafontaine (CNC); Rampart House, [67.421455 N, 140.983336 W], 18.vii.1951, 1 female, CNCD 13981, C.C. Loan (CNC); Old Crow, [67.571219 N, -139.833877 W], 2.vii.1983, 1 female, JSS 20138, R.A. Cannings (UBCZ); British Mountains, Firth River, [68.848549 N, -140.48328 W], 24.vii.1956, 1 female, CNCD 14053, R.E. Leech (CNC); Firth River, [69.207056 N, -140.071033 W], 950m, 24.vi.1984, 1 female, JSS 18367, S.G. Cannings (CNC); Kluane; Ruby Ranges; Pika Camp, [16.vi.2004 23.vi.2004, 1 female, JSS 25586, E.S. Bakker (WVSC); United States of America: Alaska: Anchorage, Anchorage County, [61.175333 N, 149.861715 W], 13.vi.1921, 1 female, JSS 19547, J.M. Aldrich (CNC); Matanuska, Matanuska-Susitna County, [61.541945 N, -149.229674 W], 4.vi.1944, 1 female, JSS 19557, J. Chamberlin (CNC); Matanuska, Matanuska-Susitna County, [61.541945 N, 149.229674 W], 10.vi.1944, 1 female, JSS 19537, J.C. Chamberlin (CNC); Matanuska, Matanuska-Susitna County, [61.541945 N, -149.229674 W], 11.vi.1944, 1 female, JSS 19536, J.C. Chamberlin (CNC); Matanuska, Matanuska-Susitna County, [61.541945 N, -149.229674 W], 17.vi.1944, 1 female, JSS 19538, J.C. Chamberlin (CNC); Matanuska, Matanuska-Susitna County, [61.541945 N, -149.229674 W], 1.vi.1945, 2 females, JSS 19535, 19539, J.C. Chamberlin (CNC); Trapper Creek, 36 miles North of Willow; [62.31666667 N, -150.2166667 W], 19.vii 1984, CNCD 60064, S. \& J. Peck (CNC); Unalakleet, [63.86666667 N, -160.7833333 W], 
17.vi.1961, 1 female, CNCD 26575, B.S. Heming (CNC); Unalakleet, [63.86666667 N, -160.7833333 W], 16.viii.1961, 2 females, CNCD 26577, 26578, R. Madge (CNC); Big Delta, Lake Boleo, Southeast Fairbanks County, [64.154432 N, -145.836524 W], 19.v.1951, 1 female, CNCD 26553, W.R.M. Mason (CNC); Nome Area, Mile 55 of Kougarok Road, [65.08972222 N, -164.6722222 W], 20-26.vi.2005, 1 female, CNCD 30893, J.\&R. Skevington (CNC);Nome Area, Mile 17 of Kougarok Road, [64.70611111 N, -165.2972222 W], 21-23.vi.2005, 2 females, CNCD 30881, 30902, J.\&R. Skevington (CNC); Nome Area, Mile 17 of Kougarok Road, [64.70611111 N, 165.2972222 W], 26-28.vi.2005, 2 females, CNCD 30895, 30897, 30898, J.\&R.

Skevington (CNC); Colorado: Chambers Lake, [40.603663 N, -105.853645 W], 3.viii.1932, 1 female, JSS 20714, Chas L. Fluke (CSUC); Idaho: Keene Valley, [44.18333333 N, -73.78333333 W], 25.v.1910, JSS 21351, H. Notman (MCZ); Maine: Bar Harbor, [44.38333333 N, -68.2 W], 1 female, JSS 21348, (MCZ); Dryden, Franklin County, [44.586729 N, -70.209783 W], 20.vii.1959, 1 male, CNCD 26734, G.H. Heinrich (CNC); Massachusetts: Hampshire County, [42.335786 N, -72.644117 W], .vi.1922, 1 male, CNCD 26739, P.A. Readio (CNC); Melrose Highlands, Middlesex County, [42.470929 N, -71.061441 W], 16.vi.1911, 1 male, CNCD 26740, J.D. Tothill (CNC); Michigan: Manistee Lake, [44.24749 N, -86.30471 W], 22.v.1955, 1 male, JSS 21138, R.W. Hodges (MSUC); Sagola, [46.09028 N, -88.0775 W], 6.vi.1983, 1 female, JSS 21136, M. Arduser (MSUC); Isle Royale, [48 N, -88.83333333 W], 3.vii.1957, 1 female, JSS 21142, R.W. Hodges (MSUC); Isle Royale, [48 N, -88.83333333 W], 15.vii.1957, 2 females, ISS 21144, ISS 21147, R.W. Hodges (MSUC); Isle Royale, [48 N, -88.83333333 W], 18.vii.1957, 1 female, JSS 21143, R.W. Hodges (MSUC); Isle 
Royale, [48 N, -88.83333333 W], 11.viii.1957, 1 female, JSS 21141, R.W. Hodges (MSUC); Kalkaska County, Michigan, 14.vi.1949, 1 female, JSS 21139, R.R. Dreisbach (MSUC); Kalkaska County, Michigan, 12.vi., 1 female, JSS 21140, R.R. Dreisbach (MSUC); Chippewa County, Michigan, 3.vi.1957, 2 females, JSS 21145, 21146, R. \& K. Dreisbach (MSUC); Clare County, Michigan, 16.v.1951, 1 female, JSS 21119, R.R. Dreisbach (MSUC); Clare County, Michigan, 23.v.1959, 2 females, JSS 21148, 21149, R. Dreisbach (MSUC); New Hampshire: Franconia, Rt.3 Fanconia North, [44.209935 N, -71.685178 W], 20-21.vi.1972, 1 female, USNM ENT 258066, B.J. \& F.C. Thompson (USNM); Pittsburg, Rt.3 Connecticut Lakes, [45.104264 N, -71.259207 W], 22-24.vi.1972, 4 females, USNM ENT 258067-258070, B.J. \& F.C. Thompson (USNM); Jaffrey, Cheshire County, [42.814957 N, -72.022399 W], 1941, 1 male, CNCD 26741, C.W. Johnson (CNC); Bretton Woods, [44.25 N, -71.43333333 W], 27.vi., 1 male, CNCD 26742, C.W. Johnson (CNC); Mount Washington, Coos County, [44.270481 N, -71.30255 W], 6.vii., 1 male, CNCD 26735, (CNC); Lancaster Mt. Prospect, [44.449317 N, -71.570888 W], 625m, 19.vi.1982, 1 female, JSS 20780, J.R. Vockeroth (CNC); New Mexico: Sierra Blanca, Lincoln Co.; [33.478065 N, 105.552907 W], 2957m, 10-26.vi.1979, 1 female, JSS 18336, S. \& J. Peck (CNC); New York: Oliverea, Ulster County, [42.065369 N, -74.460152 W], 18.vi.1918, 1 male, CNCD 26745, (CNC); Maplecrest, Catskill Mts., [42.275919 N, -74.186809 W], 18.vi.1927, JSS 19255, F.M. Schott (FMNH); Heart Lake, Mount Jo, Essex Co., [44.182434 N, -73.966816 W], 13.vi.1935, 1 male, CNCD 26743, C.W. Johnson (CNC); North Carolina: Shining Rock Wild. Area, [35.355 N, , -82.84388889 W], 23.v.1993, 1 female, JSS 20807, Kondratieff \& Kirchner (CSUC); Pisgah National 
Forest, 10km SE Linville on [R---] Road, [36 N, $-81.8 \mathrm{~W}$ ], 760m, 25.v.1999, 1 female, JSS 20988, M.I. Erwin (MHPC]; 13km W Boone on Blue Ridge Pkwy, [36.23333333 N, -81.51666667 W], 1050m, 24.v.1999, 1 female, JSS 20987, M.I. Erwin (MHPC); 13km W Boone on Blue Ridge Pkwy, [36.23333333 N, -81.51666667 W], 1050m, 25.v.1999, 1 male, JSS 20983, M.I. Erwin (MHPC); Pennsylvania: Black Moshannon State Park, [40.896041 N, -78.044398 W], 13.v.1990, 1 male, USNM ENT 258081, FC Thompspn (USNM); Centre Co., Bear Meadows Natural Area, 11.v.1983, 1 male,

CNCD 5458, F.C. Thompson (USNM); Utah: Cache Co., Tony Grove Crk, [41.892197 N, -111.634869 W], 8-15.vii.1994, 1 female, JSS 24642, W.J. Hanson (EMUS);

Vermont: 5 Miles West of Bloomfield, Essex Co., [44.753513 N, -71.727243 W], 28.vi.1972, 1 female, JSS 18338, H.J. Teskey (CNC); 6 Miles West of Bloomfield, Essex Co., [44.753513 N, -71.727243 W], 28.vi.1972, 1 female, JSS 18339, H.J.

Teskey (CNC); Virginia: Smyth Co., Hurricane Creek, 22.v.1981, 1 male, CNCD 5456, F.C. \& B.J. Thompson (USNM); West Virginia: Standard, off I-77, [38.136111 N, 81.400556 W], 6.v.2005, 1 female, JSS 20692, B. Kondratieff, R. Zuelig, R.F. Kirchner (CSUC).

4.4 Discussion

Nearctic Dasysyrphus show low rates of intraspecific genetic variation for COI (Table 2). Almost all species have a maximum intraspecific variation of less than $1 \%$. Where the intraspecific variation is higher, it is likely that cryptic species still 
exist. In terms of interspecific variation, seven species have a $>2 \%$ genetic divergence from their nearest neighbour (Table 2). This $2 \%$, or greater, difference between species was found to exist in more than $98 \%$ of species pairs examined by Hebert et al. (2003) but was likely biased upward as their work was not revisionary. Twelve species of Nearctic Dasysyrphus have $<2 \%$ genetic divergence from their nearest neighbour, with the majority of those being less than $1 \%$ (Table 2). Even though low divergence values exist for many species, there is still strong evidence for the species concepts put forth above.

Nearctic Dasysyrphus pauxillus s.l. is here split into four species with two names being resurrected from synonymy (D. laticaudus and D. pacificus) and one species being recognized in this region that was previously only known from the Palaearctic (D. nigricornis). Dasysyrphus laticaudus, D. pacificus and D. pauxillus form a clade as expected; however, D. nigricornis is not directly related (Figs $2 \& 3$ ). All four of these species have an intraspecific variation value below $0.5 \%$ (Table 2). Dasysyrphus nigricornis and D. occidualis have identical COI barcoding regions, rendering them indistinguishable using COI data. They both have low mean intraspecific variation $(0.31 \%$ and $0.11 \%$ respectively) and they are both each other's nearest neighbour with a distance to the nearest neighbour of $0 \%$.

Further investigation should be done into $D$. bilineatus. This Palaearctic/Oriental species shows great variation in size. It has a maximum intraspecific variation of $0.93 \%$, which is higher than the maximum intraspecific 
variation of most other species (ignoring the complex of intrudens and lotus and venustus, which may also be two species each; Table 2). However, at just below $1 \%$, this may just be a species with high intraspecific variation. This species falls beyond the scope of this paper and too few specimens were available for both morphological and genetic study to draw more compelling conclusions.

In this study, the intrudens complex is split off from venustus. Vockeroth synonymized the intrudens complex under venustus in 1986, treating it as a highly morphologically variable group. It is difficult to use morphology, ecology and geography to help delimit the boundaries of species in venustus sensu Vockeroth. Genitalia are extremely similar, in all specimens of the intrudens complex and venustus, even though you can identify venustus by the more straight abdominal maculae, as outlined above. Within the intrudens complex, no congruent patterns exist between morphology, COI, ecology and range. Morphological traits often vary more within a genetic cluster than between clusters. There is some congruence between COI data and putative species range, with clusters representing typical cordilleran, boreal or Carolinian species patterns; however, none of these patterns are reflected in morphology. More intense work needs to focus on this group to attempt to resolve the true nature of the species and their relations. If more DNA regions are examined they may provide evidence that shows a clearer pattern. With COI providing so few molecular synapomorphies to support Dasysyrphus species concepts it is not surprising that more rapidly evolving markers are required to help sort out this remaining complex of species. Perceived morphological variation 
will undoubtedly be minimized when more rapidly evolving genes are applied to the problem. It would be interesting to figure out how species of Dasysyrphus that are so similar perceive each other and maintain reproductive isolation.

Vockeroth (1969) pointed out, based on morphology, that Dasysyrphus is one of several groups of syrphines with low interspecific variation and that species were difficult to distinguish. He noted that they tend to have low variation in characters like abdominal colour pattern, shape and size, thoracic colour, head shape and face colour, antennal shape, wing shape, hair characters and structure of male genitalia. Not surprisingly, we now see that their COI sequences show low interspecific variation as well. This low interspecific variation makes delimiting species a difficult task. In the case of pauxillus and allies, COI helped to clarify the distinctions between species. However, in the case of the intrudens complex it has been of no use. Although some progress has been made, such as separating the intrudens complex from venustus, this group still needs work. Despite a failure to resolve the intrudens group problem, bringing new data to Dasysyrphus has clarified most species concepts and should allow research on species in the genus to move forward.

A total evidence approach was useful in delimiting species of Dasysyrphus. Neither COI nor morphology alone was able to resolve all species. Dasysyrphus nigricornis and D. occidualis had identical barcodes and would not have been distinguished if not for morphology. Likewise, sorting the pauxillus sensu Vockeroth 
specimens based on morphology alone would likely have not resulted in the same degree of delimitation due to their extremely similar habitus and very few and subtle differences. While both of these data sets combined were not able to resolve the $D$. intrudens complex, the combination of COI and morphology worked for the rest of Dasysyrphus. Likely, with more molecular data sets the intrudens complex will be able to be resolved. Using multiple data sets and taking a total evidence approach should be used when possible, as each data set can provide information that another might not.

References

American Museum of Natural History (2005) AMNH Invertebrate Zoology Type Specimens Database. http://research.amnh.org/iz/types db/ (Feb. 27, 2012). Barkalov, A.V. (2007) Hoverflies of the genus Dasysyrphus Enderlein, 1937 (Diptera, Syrphidae) from the Urals, Siberia and the Far East. Euroasian Entomological Journal, 6, 273-298.

Bartsch, H., Binkiewicz, E., Råden, A. \& Nasibov, E. (2009) Nationalnyckeln till Sverigesflora och fauna. Tvåvingar: Blomflugor: Syrphinae. Diptera: Syrphidae: Syrphinae. ArtDatabanken, SLU, Uppsala.

Becker, T. (1921) Neue Dipteren meiner Sammlung. I. Syrphidae. Mitteilungen aus dem Zoologischen Museum in Berlin, 10, 1-93. 
Beckemeyer, R.J. (2009). Samuel Wendell Williston - The first and foremost Kansas dipterist. Transactions of the Kansas Academy of Science, 112(4), 225-228.

Bicik, V. \& Laska, P. (1996) Revision of type material of Scaeva obscura Zetterstedt, 1838 (Diptera, Syrphidae). Acta universitatis Palackianae Olomucensis Facultas Rerum Naturalium Biologica, 33, 7-14.

Brouillet, L., Coursol, F., Meades, S.J., Favreau, M., Anions, M., Bélisle, P. \& Desmet, P. (2010) VASCAN, the Database of Vascular Plants of Canada. http://data.canadensys.net/vascan/(May 1,2012).

Brown, R.W. (1956) Composition of Scientific Words. Smithsonian Institution Press, Washington, D.C.

Byers, G.W., Blank, F., Hanson, W.J., Beneway, D.F. \& Fredrichson, R.W. (1962) Catalogue of they types in the Snow Entomological Museum. Part III (Diptera). University of Kansas Science Bulletin, 43, 131-181.

Cole, F.R. \& Lovett, A.L. (1919) New Oregon Diptera. Proceedings of the California Academy of Sciences, 9, 221-255.

Cumming, J.M. \& Wood, D.M. (2009) Adult morphology and terminology. In: Manual of Central American Diptera, 2-50.

Curran, C.H. (1921) A revision of Syrphus species belonging to the ribesii group (Dipt.). The Canadian Entomologist, 152-160.

Curran, C.H. (1925) Contribution to a Monograph of the American Syrphidae from North of Mexico. Bulletin of the University of Kansas - Science Bulletin, XV, 1283.

Darwin, C. (1859) On the Origin of Species. Random House Inc, New York, NY. 
De Geer, C. (1776) Mémoires pour servir à l'histoire des Insects. Hosselberg, Stockholm, Sweden, $523 \mathrm{pp}$.

De Queiroz, K. (2005) Ernst Mayr and the modern concept of species. Proceedings of the National Academy of Sciences, 102, 6600-6607.

De Queiroz, K. (2007) Species concepts and species delimitation. Systematic Biology, $56,879-886$.

Dillon, S. \& Fjeldså, J. (2005) The implications of different species concepts for describing biodiversity patterns and assessing conservation needs for African birds. Ecography, 28, 682-692.

Doczkal, D. (1996) Hoverflies from Germany: first records and little known species (Diptera, Syrphidae). Volucella, 2, 36-62.

Du Rietz, G.E. (1930) The fundamental units of biological taxonomy. Svensk Botanisk Tidskrift, 24, 333-428.

Dusek, J. \& Laska, P. (1967) Versuch zum aufbau eines Naturlichen Systems mitteleuropaischer Arten der Unterfamilie Syrphinae (Diptera). Acta scientiarum naturalium Academiae bohemoslovacae Brno, 1, 349-390.

Dusek, J. \& Laska, P. (1969) Scaeva arcuata Fallen, 1817 (Insecta, Diptera): Proposed suppression of the specific name under the plenary powers. Z.N.(S.) 1885. Bulletin of Zoological Nomenclature, 26, 171-173.

Dusek, J. \& Laska, P. (1974) Influence of temperature during pupal development on the colour of syrphid adults (Syrphidae, Diptera). Folia Facultatis Scientiarum Naturalium Universitatis Purkyninae Bruennsis, 77-81. 
Enderlein, G. (1938) Beiträge zur kenntnis der Syrphiden. Sitzungsberichte der Gesellschaft Naturforschender Freunde Berlin, 1937, 192-237.

Evenhuis, N.L. (2012)) Abbreviations for Insect and Spider Collections of the World. http://hbs.bishopmuseum.org/codens/codens-inst.html (February 3, 2012).

Fallén, F.C. (1817) Syrphici Sveciae. In: Diptera Sveciae / descripta a Carlo Frederico Fallén. Literis Berlingianis, Lundae, pp 1-14 [1816.06.08], 15-22 [1816.06.08], $23-30[1817.05 .10], 31-42[1817.05 .20], 43-54$ [1817.05.21], 55-62. $[1817.05 .22]$.

Farris, J.S., Albert, V.A., Källersjö, M., Lipscomb, D. \& Kluge, A.G. (1996) Parsimony jackknifing outperforms neighbor-joining. Cladistics, 12, 99-124.

Flint, O.S.J., Menke, A.S., Thompson, F.C. \& Ward, R.A. (1976) The United States National Entomological Collections. Smithsonian Institution Press, Washington, D.C: .

Fluke, C.L. (1933) Revision of the Syrphus flies of America north of Mexico (Diptera, Syrphidae, Syrphus S.L.) Part 1. Transactions of the Wisconsin Academy of Sciences, Arts and Letters, 28, 63-127.

Folmer, O., Black, M., Hoeh, W., Lutz, R. \& Vrijenhoek, R. (1994) DNA primers for amplifcation of mitochondrial cytochrome c oxidase subunit I from diverse metazoan invertebrates. Molecular Marine Biology and Biotechnology, 3, 294299.

Frey, R. (1950) Dipterfaunan vid Tana älv i Utsjoki sommaren 1949. Notulae Entomologica, 30, 5-17. 
Ghorpade, K. (1994) Diagnostic keys to new and known genera and species of Indian subcontinent Syrphini (Diptera: Syrphidae). Colemania: Insect Biosystematics, 3, 1-15.

Gibson, J.F., Kelso, S., Jackson, M.D., Kits, J.H., Miranda, G.F.G. \& Skevington, J.H. (2011) Diptera-specific polymerase chain reaction amplification primers of use in molecular phylogenic research. Annals of the Entomological Society of America, 104, 976-997.

Gibson, J.F., Skevington, J.H. \& Kelso, S. (2010) Placement of Conopidae (Diptera) within Schizophora based on mtDNA and nrDNA gene regions. Molecular Phylogenetics and Evolution, 56, 91-103.

Goeldlin de Tiefenau, P. (1974) Contribution a l'etude systematique et ecologique des Syrphidae (Dipt.) de la Suisse occidentale. Mitteilungen der Schweizerischen Entomologischen Gesellschaft, 47, 151-251.

Goffe, E.R. (1944) Some changes in generic nomenclture in Syrphidae (Diptera). Entomologist's Monthly Magazine, 80, 128-132.

Hajibabaei, M., deWaard, J.R., Ivanova, N.V., Ratnasingham, S., Dooh, R.T., Kirk, S.L., Mackie, P.M. \& Hebert, P.D.N. (2005) Critical factors for assembling a high volume of DNA barcodes. Philosophical Transactions of the Royal Society B, $360,1959-1967$.

Hebert, P.D.N., Ratnasingham, S. \& de Waard, J.R. (2003) Barcoding animal life: cytochrome c oxidase subunit 1 divergence among closely related species. Proceedings of the Royal Society B, 270, S96-S99. 
Hennig, W. (1966) Phylogenetic Systematics. University of Illinois Press, Champaign, IL.

Hey, J. (2006) On the failure of modern species concepts. Trends in Ecology and Evolution, 21, 447-450.

Hine, J.S. (1922) Descriptions of Alaskan Diptera of the family Syrphidae. Ohio Journal of Science, 22, 143-147.

Huo, K.-K., Zhang, H.-J. \& Zheng, Z.-M. (2005) Two new species of Dasysyrphus (Diptera, Syrphidae) from China, with a key to species from China. Acta Zootaxonomica Sinica, 30, 847-851.

ICZN: International Commission on Zoological Nomenclature of 1972 (1972) Opinion 978: Scaeve arcuate Fallén, 1817 (Insecta, Diptera): Suppressed under the plenary powers. Bulletin of Zoological Nomenclature, 29, 9-10.

Krpac, V.T., Vujic, A., Simic, S., Radenkovic, S. \& Lazarevska, S. (2009) Revision of the genus Dasysyrphus Enderlein, 1938 (Diptera: Syrphidae) in the fauna of Macedonia. Kragujevac Journal of Science, 31, 103-108.

Laska, P. \& Bicik, V. (1996) On the problems of the species Dasysyrphus venustus (Zetterstedt) and D. hilaris (Meigen) (Dipt., Syrphidae). Entomologist's Monthly Magazine, 132, 305-309.

Maddison, W.P. \& Maddison, D.R. (2010) Mesquite: a modular system for evolutionary analysis Version 2.73 (build 544). http://mesquiteproject.org (February 3, 2012).

Matsumura, S. (1918) New species of the economic Syrphidae of Japan. Journal of the College of Agriculture Hokkaido Imperial University, 8, 1-31. 
Matsumura, S. \& Adachi, J. (1917) Synopsis of the economic Syrphidae of Japan (pt. III). The Entomological Magazine, Kyoto, 3, 14-46.

Mayr, E. (1942) Systematics and the Origin of Species. Columbia University Press, New York, NY.

Meigen, J.W. (1822) Systematische Beschreibung der bekannten europaischen zweiflugeligen Insekten. Dritter Theil. Schulz-Wundermann, Hamm, Germany, $416 \mathrm{pp}$.

Mengual, X., Ståhls, G. \& Rojo, S. (2008) First phylogeny of predatory flower flies (Diptera, Syrphidae, Syrphinae) using mitochondrial COI and nuclear $28 \mathrm{~S}$ rRNA genes: conflict and congruence with the concurrent tribal classification. Cladistics, 24, 543-562.

Miller, M.A., Pfeiffer, W. \& Schwartz, T. (2010) Creating the CIPRES Science Gateway for inference of large phylogenetic trees in Proceedings of the Gateway Computing Environments Workshop (GCE), 14 Nov. 2010, New Orleans, LA pp $1-8$.

Mutin, V.A. \& Barkalov, A.V. (1999) Syrphidae. In: Lehr, P.A. (Ed.) Key to the Insects of the Russian Far East. Nauka Publishing House, Leningrad, pp. 655.

Neilsen, T.R. (1998) Hoverflies (Dipt., Syrphidae) in the arctic Pasvik valley, Norway. Fauna Norvegica Series B, 45, 83-92.

Nylander, J.A.A. (2004) MrModetest v2. Program distributed by the author. Evolutionary Biology Centre, Uppsala University. 
Osten Sacken, C.R. (1875) On the North American species of the genus Syrphus (in the narrowest sense). Proceedings of the Boston Society of Natural history, 18, 135-153.

Osten Sacken, C.R. (1877) Western Diptera: Descriptions of new genera and species of Diptera from the region west of the Mississippi and especially from California. Bulletin of the United States Geological and Geographical Survey of the Territories, 3, 189-354.

Pape, T. \& Thompson, F. C. (2010a) Dasysyrphus Enderlein. Systema Dipterorum, Version 1.0. 44 work records (not peer-reviewed material). http://www.diptera.org/ (January 17, 2012).

Pape, T. \& Thompson, F. C. (2010b) Dasysyrphus albostriatus. Systema Dipterorum, Version 1.0. 1 work record (not peer-reviewed material). http://www.diptera.org/ (December 15, 2011).

Pape, T. \& Thompson, F. C. (2010c) Dasysyrphus venustus. Systema Dipterorum, Version 1.0. 1 work record (not peer-reviewed material). http://www.diptera.org/ (December 15, 2011).

Peck, L.V. (1974) New species of hover flies (Diptera, Syrphidae) in the Palearctic fauna. Entomologicheskoe Obozrenie, 53, 903-915.

Peck, L.V. (1988) Family Syrphidae. In: Soos, A. \& Papp, L. (Eds.) Catalogue of Palaearctic Diptera. Volume 8. Syrphidae-Conopidae. Akadémiai Kiadó, Budapest, Hungary, pp. 11-230. 
Penney, H.D., Hassal, C., Skevington, J.H., Abbott, K.R. \& Sherratt, T.N. (2012) A comparative analysis of the evolution of imperfect mimicry. Nature, 483, 461464.

Ratnasingham, S. \& Hebert, P.D.N. (2007) BOLD : The Barcode of Life Data System (www.barcodinglife.org). Molecular Ecology Notes, 7, 355-364.

Reemer, M. (2002) Dasysyrphus lenensis in the Netherlands (Diptera: Syrphidae). Nederlandse Faunistische Mededelingen, 17, 13-18.

Rojo, S., Gilbert, F.S., Marcos-Garcia, M.A., Nieto, J.M. \& Mier, M.P. (2003) A World Review of Predatory Hoverflies (Diptera, Syrhidae: Syrphinae) and Their Prey. CIBIO Ediciones, Alicante, Spain,pp.

Ronquist, F., Huelsenbeck, J. \& Teslenko, M. (2011) Draft MrBayes version 3.2 Manual: Tutorials and Model Summaries. http://mrbayes.sourceforge.net/mb3.2 manual.pdf (March 2, 2012).

Rotheray, G. \& Gilbert, F. (2011) The Natural History of Hoverflies. Forrest Text, Cardigan, UK.

Rotheray, G.E. (1993) Colour Guide to Hoverfly Larvae (Diptera, Syrphidae). Derek Whiteley, Sheffield, England, $156 \mathrm{pp}$.

Shorthouse, D.P. (2010) SimpleMappr, an online tool to produce publication-quality point maps. http://www.simplemappr.net (January 5, 2012).

Simpson, G.G. (1951) The species concept. Evolution, 5, 285-298.

Snow, W.A. (1892) Notes and Descriptions of Syrphidae. Kansas University Quarterly, 1, 33-38. 
Snow, W.A. (1895) Diptera of Colorado and New Mexico. Kansas University Quarterly, 3, 225-247.

Sorenson, M.D. \& Franzosa, E.A. (2007) TreeRot, version 3. Boston University, Boston, MA.

Speight, M.C.D. (2011) Species accounts of European Syrphidae (Diptera), Glasgow, 2011. Syrph the Net Publications, Dublin, 285 pp.

Stone, A., Sabrosky, C.W., Wirth, W.W., Foote, R.H. \& Coulson, J.R. (1965) A catalog of the Diptera of America north of Mexico. U.S. Dep. Agric. Handb., Washington D.C.

Stubbs, A.E. \& Falk, S. (2002) British hoverflies: an illustrated identification guide. Second edition. British Entomological and Natural History Society, 1-469 pp.

Swofford, D. L. (2003) PAUP*. Phylogenetic Analysis Using Parsimony ( ${ }^{*}$ and Other Methods) Version 4.0. Sinauer Associates, Sunderland, MA.

Thompson, F.C. (1999) A key to the genera of the flower flies (Diptera: Syrphidae) of the Neotropical Region including descriptions of new genera and species and a glossary of taxonomic terms. Contributions on Entomology, International, 3 , $322-378$.

Thompson, F.C. \& Nielsen, T.R. (1984) Synonymic notes on some Palaearctic species of Dasysyrphus Enderlein (Diptera: Syrphidae)

Thompson, F.C. \& Pont, A. (1994) Systematic Database of Musca Names (Diptera). Theses Zoologicae, 20, 221.

USDA, NRCS (2012) The PLANTS Database. National Plant Data Team, Greensboro, NC 27401-4901 USA. http://plants.usda.gov (January 9, 2012). 
van Veen, M.P. (2004) Hoverflies of Northwest Europe: Identification keys to the Syrphidae. KNNV Publishing, Utrecht, The Netherlands.

Vockeroth, J.R. (1969) A revision of the genera of the Syrphini (Diptera: Syrphidae). Memoirs of the Entomological Society of Canada, 62, 1-176.

Vockeroth, J.R. (1986) Nomenclatural notes on Nearctic Eupeodes (including Metsyrphus) and Dasysyrphus (Diptera: Syrphidae). Canadian Entomologist, $118,199-204$.

Vockeroth, J.R. (1992) The flower flies of the subfamily Syrphinae of Canada, Alaska and Greenland. Canada Communications Group - Publishing, Ottawa, 456 pp.

Wahlberg, N. (2010) The Nymphalidae systematics group-molecular methods. http://nymphalidae.utu.fi/Nymphalidae/Molecular.htm (February 4, 2012).

Wheeler, Q.D. \& Platnick, N.I. (2000) A Defense of the Phylogenetic Species Concept (sensu Wheeler and Platnick). In: Wheeler, Q.D. \& Meier, R. (Eds.) Species Concepts and Genetic Theory: A Debate. Columbia University Press, New York, NY, pp. 55-69.

Williston, S.W. (1887) Synopsis of the North American Syrphidae. Bulletin of the United States National Museum, 31, 1-335.

Zetterstedt, J.W. (1842) Diptera Scandinaviæ disposita et descripta. ex Officina Lundbergiana, sumtibus auctoris, Lundæ. 
Table 1: Voucher information for Dasysyrphus species, including the geographc origin of the specimen and its unique voucher number.

\begin{tabular}{|c|c|c|c|}
\hline Species & Country & $\begin{array}{l}\text { Geographic Origin } \\
\text { State }\end{array}$ & Voucher Number \\
\hline Dasysyrphus albostriatus & Germany & & JSS 20734 \\
\hline Dasysyrphus amalopis & Canada & Manitoba & 10PROBE-13673 \\
\hline Dasysyrphus amalopis & Canada & Manitoba & 10PROBE-13805 \\
\hline Dasysyrphus amalopis & Canada & Manitoba & 10PROBE-10909 \\
\hline Dasysyrphus amalopis & Canada & Manitoba & CNCD 9607 \\
\hline Dasysyrphus amalopis & Canada & Manitoba & 07PROBE-04723 \\
\hline Dasysyrphus amalopis & Canada & Manitoba & 09PROBE-IW0176 \\
\hline Dasysyrphus amalopis & Canada & Manitoba & PROBE-TW0025 \\
\hline Dasysyrphus amalopis & Canada & Manitoba & 10PROBE-15042 \\
\hline Dasysyrphus amalopis & Canada & Manitoba & 10PROBE-14269 \\
\hline Dasysyrphus amalopis & Canada & Manitoba & 10PROBE-15283 \\
\hline Dasysyrphus amalopis & Canada & Yukon Territory & CNCD 13587 \\
\hline Dasysyrphus bilineatus & Russia & Primorye & JSS 21000 \\
\hline Dasysyrphus bilineatus & South Korea & & JSS 20997 \\
\hline Dasysyrphus bilineatus & South Korea & Kangwon-do & JSS 20995 \\
\hline Dasysyrphus bilineatus & South Korea & Kangwon-dó & JSS 20996 \\
\hline Dasysyrphus creper & Canada & Alberta & ISS 18293 \\
\hline Dasysyrphus creper & Canada & British Columbia & JSS 18368 \\
\hline Dasysyrphus creper & Canada & British Columbia & RBCM ENT $988-5672$ \\
\hline Dasysyrphus creper & Canada & British Columbia & CNCD 13631 \\
\hline Dasysyrphus creper & United States & Arizona & USNM ENT 00258093 \\
\hline Dasysyrphus creper & United States & Arizona & JSS 20683 \\
\hline Dasysyrphus creper & United States & California & JSS 20950 \\
\hline Dasysyrphus creper & United States & California & JSS 20956 \\
\hline Dasysyrphus creper & United States & California & JSS 20951 \\
\hline Dasysyrphus creper & United States & California & JSS 20962 \\
\hline Dasysyrphus creper & United States & California & JSS 20955 \\
\hline Dasysyrphus creper & United States & California & JSS 20954 \\
\hline Dasysyrphus creper & United States & Colorado & JSS 20684 \\
\hline Dasysyrphus creper & United States & Colorado & JSS 20685 \\
\hline Dasysyrphus creper & United States & Colorado & ISS 24626 \\
\hline Dasysyrphus creper & United States & Colorado & JSS 20809 \\
\hline Dasysyrphus creper & United States & Colorado & ISS 20806 \\
\hline Dasysyrphus creper & United States & Colorado & ISS 20805 \\
\hline Dasysyrphus creper & United States & Colorado & JSS 20682 \\
\hline Dasysyrphus creper & United States & Utah & JSS 24611 \\
\hline Dasysyrphus creper & United States & Wyoming & CNCD 5444 \\
\hline Dasysyrphus creper & United States & Wyoming & JSS 20686 \\
\hline Dasysyrphus eggeri & Spain & Granada & USNM ENT 00258107 \\
\hline Dasysyrphus intrudens sp. complex & & & JSS 20830 \\
\hline Dasysyrphus intrudens sp. complex & Austria & Steiermark & JSS 18301 \\
\hline Dasysyrphus intrudens sp. complex & Canada & Alberta & JSS 18347 \\
\hline Dasysyrphus intrudens sp. complex & Canada & Alberta & CNCD 5818 \\
\hline Dasysyrphus intrudens sp. complex & Canada & Alberta & RBCM ENT 006-006034 \\
\hline Dasysyrphus intrudens sp. complex & Canada & Alberta & ISS 21072 \\
\hline Dasysyrphus intrudens sp. complex & Canada & British Columbia & JSS 18372 \\
\hline Dasysyrphus intrudens sp. complex & Canada & British Columbia & SFU 720561 \\
\hline Dasysyrphus intrudens sp. complex & Canada & British Columbia & SFU 717487 \\
\hline Dasysyrphus intrudens sp. complex & Canada & British Columbia & SFU 717389 \\
\hline Dasysyrphus intrudens sp. complex & Canada & British Columbia & SFU 717383 \\
\hline Dasysyrphus intrudens sp. complex & Canada & British Columbia & CNCD 5816 \\
\hline Dasysyrphus intrudens sp. complex & Canada & British Columbia & SFU 717335 \\
\hline Dasysyrphus intrudens sp. complex & Canada & British Columbia & SFU 716998 \\
\hline Dasysyrphus intrudens sp. complex & Canada & British Columbia & SFU 716995 \\
\hline
\end{tabular}


Table 1: con't

\begin{tabular}{|c|c|c|c|}
\hline \multirow[t]{2}{*}{ Species } & \multicolumn{2}{|r|}{ Geographic Origin } & \multirow[t]{2}{*}{ Voucher Number } \\
\hline & Country & State & \\
\hline Dasysyrphus intrudens sp. complex & Canada & British Columbia & SFU 716919 \\
\hline Dasysyrphus intrudens sp. complex & Canada & British Columbia & SFU 716878 \\
\hline Dasysyrphus intrudens sp. complex & Canada & British Columbia & SFU 716763 \\
\hline Dasysyrphus intrudens sp. complex & Canada & British Columbia & CNCD 14312 \\
\hline Dasysyrphus intrudens sp. complex & Canada & British Columbia & SFU 716544 \\
\hline Dasysyrphus intrudens sp. complex & Canada & British Columbia & SFU 716481 \\
\hline Dasysyrphus intrudens sp. complex & Canada & British Columbia & SFU 716181 \\
\hline Dasysyrphus intrudens sp. complex & Canada & British Columbia & SFU 716133 \\
\hline Dasysyrphus intrudens sp. complex & Canada & British Columbia & SFU 715931 \\
\hline Dasysyrphus intrudens sp. complex & Canada & British Columbia & JSS 20826 \\
\hline Dasysyrphus intrudens sp. complex & Canada & British Columbia & JSS 20823 \\
\hline Dasysyrphus intrudens sp. complex & Canada & British Columbia & SFU 715868 \\
\hline Dasysyrphus intrudens sp. complex & Canada & British Columbia & SFU 715565 \\
\hline Dasysyrphus intrudens sp. complex & Canada & British Columbia & SFU 715497 \\
\hline Dasysyrphus intrudens sp. complex & Canada & British Columbia & CNCD 30911 \\
\hline Dasysyrphus intrudens sp. complex & Canada & British Columbia & CNCD 30910 \\
\hline Dasysyrphus intrudens sp. complex & Canada & British Columbia & CNCD 30907 \\
\hline Dasysyrphus intrudens sp. complex & Canada & British Columbia & CNCD 30905 \\
\hline Dasysyrphus intrudens sp. complex & Canada & British Columbia & CNCD 30904 \\
\hline Dasysyrphus intrudens sp. complex & Canada & British Columbia & JSS 20744 \\
\hline Dasysyrphus intrudens sp. complex & Canada & British Columbia & UASM 141937 \\
\hline Dasysyrphus intrudens sp. complex & Canada & Manitoba & 09PROBE-JW0140 \\
\hline Dasysyrphus intrudens sp. complex & Canada & Newfoundland and Labrador & JSS 18344 \\
\hline Dasysyrphus intrudens sp. complex & Canada & Nova Scotia & CNCD 14772 \\
\hline Dasysyrphus intrudens sp. complex & Canada & Ontario & ROMEnt Spec. No. 98537 \\
\hline Dasysyrphus intrudens sp. complex & Canada & Ontario & ROMEnt Spec. No. 98520 \\
\hline Dasysyrphus intrudens sp. complex & Canada & Ontario & ROMEnt Spec. No. 57891 \\
\hline Dasysyrphus intrudens sp. complex & Canada & Ontario & ROMEnt Spec. No. 74269 \\
\hline Dasysyrphus intrudens sp. complex & Canada & Ontario & ROMEnt Spec. No. 98517 \\
\hline Dasysyrphus intrudens sp. complex & Canada & Ontario & ROMEnt Spec. No. 98529 \\
\hline Dasysyrphus intrudens sp. complex & Canada & Ontario & ROMEnt Spec. No. 98538 \\
\hline Dasysyrphus intrudens sp. complex & Canada & Ontario & CNCD 14568 \\
\hline Dasysyrphus intrudens sp. complex & Canada & Ontario & CNCD 14602 \\
\hline Dasysyrphus intrudens sp. complex & Canada & Ontario & JSS 24634 \\
\hline Dasysyrphus intrudens sp. complex & Canada & Ontario & JSS 24624 \\
\hline Dasysyrphus intrudens sp. complex & Canada & Ontario & ISS 21360 \\
\hline Dasysyrphus intrudens sp. complex & Canada & Quebec & JSS 18343 \\
\hline Dasysyrphus intrudens sp. complex & Canada & Quebec & JSS 20800 \\
\hline Dasysyrphus intrudens sp. complex & Canada & Quebec & JSS 20796 \\
\hline Dasysyrphus intrudens sp. complex & Canada & Quebec & JSS 20794 \\
\hline Dasysyrphus intrudens sp. complex & Canada & Quebec & JSS 20792 \\
\hline Dasysyrphus intrudens sp. complex & Canada & Quebec & CNCD 14633 \\
\hline Dasysyrphus intrudens sp. complex & Canada & Quebec & CNCD 14634 \\
\hline Dasysyrphus intrudens sp. complex & Canada & Quebec & CNCD 30878 \\
\hline Dasysyrphus intrudens sp. complex & Canada & Quebec & CNCD 30877 \\
\hline Dasysyrphus intrudens sp. complex & Canada & Quebec & ISS 19738 \\
\hline Dasysyrphus intrudens sp. complex & Canada & Quebec & JSS 24620 \\
\hline Dasysyrphus intrudens sp. complex & Canada & Quebec & JSS 15331 \\
\hline Dasysyrphus intrudens sp. complex & Canada & Quebec & JSS 17748 \\
\hline Dasysyrphus intrudens sp. complex & Canada & Yukon Territory & CNCD 5814 \\
\hline Dasysyrphus intrudens sp. complex & Canada & Yukon Territory & CNCD 5815 \\
\hline Dasysyrphus intrudens sp. complex & Canada & Yukon Territory & CNCD 14030 \\
\hline Dasysyrphus intrudens sp. complex & Canada & Yukon Territory & CNCD 14033 \\
\hline Dasysyrphus intrudens sp. complex & Canada & Yukon Territory & CNCD 14034 \\
\hline Dasysyrphus intrudens sp. complex & Canada & Yukon Territory & ISS 20821 \\
\hline
\end{tabular}


Table 1: con't

\begin{tabular}{|c|c|c|c|}
\hline \multirow[t]{2}{*}{ Species } & \multicolumn{2}{|r|}{ Geographic Origin } & Voucher Number \\
\hline & Country & State & \\
\hline Dasysyrphus intrudens sp. complex & Canada & Yukon Territory & JSS 20818 \\
\hline Dasysyrphus intrudens sp. complex & Canada & Yukon Territory & JSS 20817 \\
\hline Dasysyrphus intrudens sp. complex & Canada & Yukon Territory & CNCD 14028 \\
\hline Dasysyrphus intrudens sp. complex & Canada & Yukon Territory & CNCD 14027 \\
\hline Dasysyrphus intrudens sp. complex & Canada & Yukon Territory & CNCD 14035 \\
\hline Dasysyrphus intrudens sp. complex & Canada & Yukon Territory & LEM 0014304 \\
\hline Dasysyrphus intrudens sp. complex & Canada & Yukon Territory & LEM 0014302 \\
\hline Dasysyrphus intrudens sp. complex & Canada & Yukon Territory & LEM 0014466 \\
\hline Dasysyrphus intrudens sp. complex & Canada & Yukon Territory & LEM 0014469 \\
\hline Dasysyrphus intrudens sp. complex & Germany & Baden-Wuerttemberg & JSS 18299 \\
\hline Dasysyrphus intrudens sp. complex & Norway & & CNCD 35316 \\
\hline Dasysyrphus intrudens sp. complex & Russia & & JSS 20829 \\
\hline Dasysyrphus intrudens sp. complex & United States & Alaska & JSS 18329 \\
\hline Dasysyrphus intrudens sp. complex & United States & Alaska & CNCD 30901 \\
\hline Dasysyrphus intrudens sp. complex & United States & Alaska & CNCD 30900 \\
\hline Dasysyrphus intrudens sp. complex & United States & Alaska & CNCD 30894 \\
\hline Dasysyrphus intrudens sp. complex & United States & Alaska & CNCD 30892 \\
\hline Dasysyrphus intrudens sp. complex & United States & Alaska & CNCD 30891 \\
\hline Dasysyrphus intrudens sp. complex & United States & Alaska & CNCD 30880 \\
\hline Dasysyrphus intrudens sp. complex & United States & Alaska & CNCD 30879 \\
\hline Dasysyrphus intrudens sp. complex & United States & Arizona & CNCD 5461 \\
\hline Dasysyrphus intrudens sp. complex & United States & California & JSS 20791 \\
\hline Dasysyrphus intrudens sp. complex & United States & California & JSS 20994 \\
\hline Dasysyrphus intrudens sp. complex & United States & California & JSS 20991 \\
\hline Dasysyrphus intrudens sp. complex & United States & California & JSS 19706 \\
\hline Dasysyrphus intrudens sp. complex & United States & California & JSS 20990 \\
\hline Dasysyrphus intrudens sp. complex & United States & California & JSS 20973 \\
\hline Dasysyrphus intrudens sp. complex & United States & California & JSS 20965 \\
\hline Dasysyrphus intrudens sp. complex & United States & California & JSS 20964 \\
\hline Dasysyrphus intrudens sp. complex & United States & California & JSS 20961 \\
\hline Dasysyrphus intrudens sp. complex & United States & California & JSS 20960 \\
\hline Dasysyrphus intrudens sp. complex & United States & California & JSS 20959 \\
\hline Dasysyrphus intrudens sp. complex & United States & California & JSS 20953 \\
\hline Dasysyrphus intrudens sp. complex & United States & California & ISS 20952 \\
\hline Dasysyrphus intrudens sp. complex & United States & Colorado & JSS 20691 \\
\hline Dasysyrphus intrudens sp. complex & United States & Colorado & JSS 20688 \\
\hline Dasysyrphus intrudens sp. complex & United States & Colorado & JSS 20687 \\
\hline Dasysyrphus intrudens sp. complex & United States & Colorado & CNCD 5441 \\
\hline Dasysyrphus intrudens sp. complex & United States & Colorado & JSS 24637 \\
\hline Dasysyrphus intrudens sp. complex & United States & Colorado & ISS 24621 \\
\hline Dasysyrphus intrudens sp. complex & United States & Colorado & ISS 23704 \\
\hline Dasysyrphus intrudens sp. complex & United States & Colorado & ISS 23702 \\
\hline Dasysyrphus intrudens sp. complex & United States & New Mexico & JSS 20993 \\
\hline Dasysyrphus intrudens sp. complex & United States & New Mexico & JSS 20989 \\
\hline Dasysyrphus intrudens sp. complex & United States & Oregon & JSS 18330 \\
\hline Dasysyrphus intrudens sp. complex & United States & Oregon & JSS 24641 \\
\hline Dasysyrphus intrudens sp. complex & United States & Tennessee & JSS 20799 \\
\hline Dasysyrphus intrudens sp. complex & United States & Tennessee & JSS 20798 \\
\hline Dasysyrphus intrudens sp. complex & United States & Utah & JSS 24633 \\
\hline Dasysyrphus intrudens sp. complex & United States & Utah & JSS 24632 \\
\hline Dasysyrphus intrudens sp. complex & United States & Utah & JSS 24631 \\
\hline Dasysyrphus intrudens sp. complex & United States & Utah & JSS 24619 \\
\hline Dasysyrphus intrudens sp. complex & United States & Washington & USNM ENT 00258094 \\
\hline Dasysyrphus intrudens sp. complex & United States & Washington & CNCD 5462 \\
\hline Dasysyrphus intrudens sp. complex & United States & Washington & ISS 20803 \\
\hline
\end{tabular}


Table 1: con't

Species

\begin{tabular}{l}
\hline Dasysyrphus intrudens sp. complex \\
Dasysyrphus intrudens sp. complex \\
Dasysyrphus intrudens sp. complex \\
Dasysyrphus intrudens sp. complex \\
Dasysyrphus intrudens sp. complex \\
Dasysyrphus intrudens sp. complex \\
Dasysyrphus intrudens sp. complex \\
Dasysyrphus intrudens sp. complex \\
Dasysyrphus intrudens sp. complex \\
Dasysyrphus intrudens sp. complex \\
Dasysyrphus laticaudus
\end{tabular}

Dasysyrphus laticaudus

Dasysyrphus laticaudus

Dasysyrphus laticaudus

Dasysyrphus laticaudus

Dasysyrphus laticaudus

Dasysyrphus laticaudus

Dasysyrphus laticaudus

Dasysyrphus laticaudus

Dasysyrphus laticaudus

Dasysyrphus laticaudus

Dasysyrphus laticaudus

Dasysyrphus laticaudus

Dasysyrphus laticaudus

Dasysyrphus laticaudus

Dasysyrphus laticaudus

Dasysyrphus laticaudus

Dasysyrphus laticaudus

Dasysyrphus laticaudus

Dasysyrphus laticaudus

Dasysyrphus laticaudus

Dasysyrphus lenensis

Dasysyrphus limatus

Dasysyrphus limatus

Dasysyrphus limatus

Dasysyrphus limatus

Dasysyrphus limatus

Dasysyrphus limatus

Dasysyrphus lotus

Dasysyrphus lotus

Dasysyrphus lotus

Dasysyrphus occidualis

Dasysyrphus occidualis

Dasysyrphus occidualis

Dasysyrphus occidualis

Dasysyrphus occidualis

Dasysyrphus occidualis

Dasysyrphus occidualis Dasysyrphus occidualis

Dasysyrphus occidualis

Dasysyrphus occidualis

Dasysyrphus occidualis

Dasysyrphus occidualis

Dasysyrphus nigricornis

Dasysyrphus nigricornis
Geographic Origin

Country State

United States Washington

United States Washington

United States Washington

United States Washington

United States Washington

United States Washington

United States Washington

United States Washington

United States Washington

United States Washington

Canada New Brunswick

Canada

New Brunswick
Ontario

Canada

Canada

Ontario

Ontario

Canada

Ontario

Canada

Canada

Ontario

Canada

Ontario

Canada

Ontario

Canada

Ontario

Canada

Canada

Ontario

Canada

Ontario

Ontario

Canada

Ontario

Canada

Ontario

Canada

Canada

Quebec

Quebec

Canada

Quebec

Canada

Canada

Canada

Germany

Quebec

Quebec

Quebec

Canada

Canada

Canada

Yukon Territory

Niedersachsen

Quebec

Yukon Territory

Canada

Yukon Territory

Canada

United States

Yukon Territory

Colombia

Yukon Territory

United States Arizona

Canada British Columbia

Canada British Columbia

Canada

Canada

British Columbia

Canada

Yukon Territory

United States Alaska

United States Washington

United States Washington

United States Washington

United States Washington

United States Washington

United States Washington

Canada

Manitoba
Voucher Number

JSS 20690
JSS 20689
JSS 20738
JSS 24638
JSS 20740
JSS 24640
JSS 24627
JSS 24623
JSS 24622
JSS 23707
CNCD 13898
JSS 21355
ISS 24614
JSS 24613
JSS 21356
CNCD 13858
CNCD 30883
JSS 19761
CNCD 30882
CNCD 30885
CNCD 30887
JSS 19757

ROMEnt Spec. No. 98519

ROMEnt Spec. No. 98518

ISS 18342

ISS 17647

JSS 17665

JSS 17664

ISS 17663

ISS 17662

ISS 18373

ISS 20737

JSS 20795

CNCD 13691

CNCD 13685

CNCD 13677

CNCD 13676

JSS 24607

USNM ENT 00035113

CNCD 26394

JSS 18303

CNCD 30909

CNCD 30903

CNCD 30908

ISS 20820

JSS 20819

CNCD 30899

CNCD 35311

ISS 20681

JSS 23706

JSS 23708

JSS 20804

JSS 24625

JSS 20831

CNCD 9206 
Table 1: con't

\begin{tabular}{|c|c|c|c|}
\hline Species & Country & $\begin{array}{l}\text { Geographic Origin } \\
\text { State }\end{array}$ & Voucher Number \\
\hline Dasysyrphus nigricornis & Canada & Manitoba & CNCD 9274 \\
\hline Dasysyrphus nigricornis & Canada & Manitoba & CNCD 9204 \\
\hline Dasysyrphus nigricornis & Canada & Manitoba & CNCD 9205 \\
\hline Dasysyrphus nigricornis & Canada & Northwest Territories & CNCD 13772 \\
\hline Dasysyrphus nigricornis & United States & Alaska & CNCD 30896 \\
\hline Dasysyrphus pacificus & United States & California & JSS 20974 \\
\hline Dasysyrphus pacificus & United States & California & JSS 20972 \\
\hline Dasysyrphus pacificus & United States & California & JSS 20978 \\
\hline Dasysyrphus pacificus & United States & California & JSS 20971 \\
\hline Dasysyrphus pacificus & United States & California & JSS 20970 \\
\hline Dasysyrphus pacificus & United States & California & JSS 20969 \\
\hline Dasysyrphus pacificus & United States & California & JSS 20968 \\
\hline Dasysyrphus pacificus & United States & California & JSS 20967 \\
\hline Dasysyrphus pacificus & United States & California & JSS 20966 \\
\hline Dasysyrphus pacificus & United States & California & JSS 20963 \\
\hline Dasysyrphus pacificus & United States & California & ISS 20979 \\
\hline Dasysyrphus pacificus & United States & California & JSS 20958 \\
\hline Dasysyrphus pacificus & United States & California & JSS 20980 \\
\hline Dasysyrphus pacificus & United States & California & ISS 20992 \\
\hline Dasysyrphus pacificus & United States & California & JSS 20957 \\
\hline Dasysyrphus pacificus & United States & California & JSS 20797 \\
\hline Dasysyrphus pacificus & United States & California & JSS 20977 \\
\hline Dasysyrphus pacificus & United States & California & JSS 20976 \\
\hline Dasysyrphus pacificus & United States & California & ISS 20975 \\
\hline Dasysyrphus pacificus & United States & Oregon & CNCD 5457 \\
\hline Dasysyrphus pacificus & United States & Washington. & CNCD 35313 \\
\hline Dasysyrphus pacificus & United States & Washington & CNCD 35314 \\
\hline Dasysyrphus pauxillus & Canada & Alberta & JSS 20801 \\
\hline Dasysyrphus pauxillus & Canada & British Columbia & CNCD 30906 \\
\hline Dasysyrphus pauxillus & Canada & British Columbia & CNCD 7969 \\
\hline Dasysyrphus pauxillus & Canada & Manitoba & O9PROBE-JW0197 \\
\hline Dasysyrphus pauxillus & Canada & Manitoba & 10PROBE-10249 \\
\hline Dasysyrphus pauxillus & Canada & Manitoba & 09PROBE-JW0255 \\
\hline Dasysyrphus pauxillus & Canada & Manitoba & CNCD 9198 \\
\hline Dasysyrphus pauxillus & United States & Colorado & JSS 20808 \\
\hline Dasysyrphus pauxillus & United States & Colorado & JSS 20693 \\
\hline Dasysyrphus pauxillus & United States & Colorado & JSS 20694 \\
\hline Dasysyrphus pauxillus & United States & Colorado & JSS 20810 \\
\hline Dasysyrphus pauxillus & United States & Colorado & JSS 20814 \\
\hline Dasysyrphus pauxillus & United States & Colorado & JSS 24618 \\
\hline Dasysyrphus pauxillus & United States & Colorado & CNCD 35312 \\
\hline Dasysyrphus pauxillus & United States & New Mexico & JSS 18346 \\
\hline Dasysyrphus pauxillus & United States & Utah & JSS 24615 \\
\hline Dasysyrphus pauxillus & United States & Utah & JSS 24616 \\
\hline Dasysyrphus pauxillus & United States & Utah & JSS 24617 \\
\hline Dasysyrphus pinastri & Sweden & Vasterbotten & CNCD 26454 \\
\hline Dasysyrphus pinastri & Sweden & Vasterbotten & ISS 18310 \\
\hline Dasysyrphus Sp. 19 & Costa Rica & encon & USNM ENT 00035102 \\
\hline Dasysyrphus Sp. 19 & Costa Rica & & JSS 18356 \\
\hline Dasysyrphus Sp. 19 & Costa Rica & & JSS 18351 \\
\hline Dasysyrphus Sp. 19 & Costa Rica & San lose & USNM ENT 00033245 \\
\hline Dasysyrphus Sp. 23 & South Korea & Kangwon-do & JSS 21067 \\
\hline Dasysyrphus tricinctus & Poland & & CNCD 5459 \\
\hline Dasysyrphus tricinctus & South Korea & Kangwon-do & JSS 21015 \\
\hline Dasysyrphus tricinctus & Sweden & Vasterbotten & ISS 18315 \\
\hline
\end{tabular}


Table 1; con't

\begin{tabular}{|c|c|c|c|}
\hline \multirow[t]{2}{*}{ Species } & \multicolumn{2}{|r|}{ Geographic Origin } & \multirow[t]{2}{*}{ Voucher Number } \\
\hline & Country & State & \\
\hline Dasysyrphus venustus & Canada & Manitoba & CNCD 9582 \\
\hline Dasysyrphus venustus & Canada & Manitoba & CNCD9582 \\
\hline Dasysyrphus venustus & Canada & Manitoba & CNCD 9495 \\
\hline Dasysyrphus venustus & Canada & Manitoba & HLC-26539 \\
\hline Dasysyrphus venustus & Canada & Manitoba & JSS 24644 \\
\hline Dasysyrphus venustus & Canada & Nova Scotia & CNCD 14759 \\
\hline Dasysyrphus venustus & Canada & Ontario & ROMEnt Spec. No. 57889 \\
\hline Dasysyrphus venustus & Canada & Ontario & ROMEnt Spec. No. 57888 \\
\hline Dasysyrphus venustus & Canada & Ontario & ROMEnt Spec. No. 57887 \\
\hline Dasysyrphus venustus & Canada & Ontario & ROMEnt Spec. No. 98521 \\
\hline Dasysyrphus venustus & Canada & Ontario & ROMEnt Spec. No. 98536 \\
\hline Dasysyrphus venustus & Canada & Ontario & ROMEnt Spec. No. 98535 \\
\hline Dasysyrphus venustus & Canada & Ontario & ROMEnt Spec. No. 98526 \\
\hline Dasysyrphus venustus & Canada & Ontario & ROMEnt Spec. No. 98540 \\
\hline Dasysyrphus venustus & Canada & Ontario & ROMEnt Spec. No. 98530 \\
\hline Dasysyrphus venustus & Canada & Ontario & JSS 21369 \\
\hline Dasysyrphus venustus & Canada & Ontario & JSS 21371 \\
\hline Dasysyrphus venustus & Canada & Ontario & ISS 21385 \\
\hline Dasysyrphus venustus & Canada & Ontario & JSS 21394 \\
\hline Dasysyrphus venustus & Canada & Ontario & JSS 21397 \\
\hline Dasysyrphus venustus & Canada & Ontario & CNCD 30890 \\
\hline Dasysyrphus venustus & Canada & Ontario & ROMEnt Spec. No. 98534 \\
\hline Dasysyrphus venustus & Canada & Ontario. & CNCD 30889 \\
\hline Dasysyrphus venustus & Canada & Ontario & CNCD 30888 \\
\hline Dasysyrphus venustus & Canada & Ontario & ROMEnt Spec. No. 98527 \\
\hline Dasysyrphus venustus & Canada & Ontario & ROMEnt Spec. No. 98528 \\
\hline Dasysyrphus venustus & Canada & Ontario & ROMEnt Spec. No. 98539 \\
\hline Dasysyrphus venustus & Canada & Ontario & ROMEnt Spec. No. 98533 \\
\hline Dasysyrphus venustus & Canada & Ontario & ROMEnt Spec. No. 98531 \\
\hline Dasysyrphus venustus & Canada & Ontario & ROMEnt Spec. No. 98541 \\
\hline Dasysyrphus venustus & Canada & Ontario & ROMEnt Spec. No. 98542 \\
\hline Dasysyrphus venustus & Canada & Ontario & ROMEnt Spec. No. 98524 \\
\hline Dasysyrphus venustus & Canada & Ontario & ROMEnt Spec. No. 98522 \\
\hline Dasysyrphus venustus & Canada & Ontario & ROMEnt Spec. No. 98523 \\
\hline Dasysyrphus venustus & Canada & Ontario & ROMEnt Spec. No. 98525 \\
\hline Dasysyrphus venustus & Canada & Ontario & ROMEnt Spec. No. 98532 \\
\hline Dasysyrphus venustus & Canada & Ontario & CNCD 14567 \\
\hline Dasysyrphus venustus & Canada & Quebec & CNCD 68 \\
\hline Dasysyrphus venustus & Canada & Quebec & JSS 20793 \\
\hline Dasysyrphus venustus & Canada & Quebec & CNCD 8658 \\
\hline Dasysyrphus venustus & Canada & Quebec & CNCD 8656 \\
\hline Dasysyrphus venustus & Canada & Quebec & CNCD 8675 \\
\hline Dasysyrphus venustus & Canada & Quebec & CNCD 14607 \\
\hline Dasysyrphus venustus & Canada & Quebec & CNCD 14611 \\
\hline Dasysyrphus venustus & Germany & Niedersachsen & JSS 20735 \\
\hline Dasysyrphus venustus & South Korea & Kangwon-do & JSS 21016 \\
\hline Dasysyrphus venustus & South Korea & Kangwon-do & JSS 21060 \\
\hline Dasysyrphus venustus & South Korea & Kangwon-do & JSS 21057 \\
\hline Dasysyrphus venustus & South Korea & Kangwon-do & JSS 21050 \\
\hline Dasysyrphus venustus & South Korea & Kangwon-do & JSS 21055 \\
\hline Dasysyrphus venustus & Spain & Castilla y Leon & ISS 18324 \\
\hline Dasysyrphus venustus & United States & Alaska & CNCD 30902 \\
\hline Dasysyrphus venustus & United States & Alaska & CNCD 30897 \\
\hline Dasysyrphus venustus & United States & Alaska & CNCD 30895 \\
\hline Dasysyrphus venustus & United States & Alaska & CNCD 30893 \\
\hline
\end{tabular}


Table 1: con't

\begin{tabular}{llll}
\multicolumn{1}{c}{ Species } & Country & Geographic Origin & Voucher Number \\
\hline Dasysyrphus venustus & United States & Alaska & CNCD 30881 \\
Dasysyrphus venustus & United States & North Carolina & JSS 20983 \\
Dasysyrphus venustus & United States & North Carolina & ISS 20985 \\
Dasysyrphus venustus & United States & North Carolina & JSS 20987 \\
Dasysyrphus venustus & United States & North Carolina & JSS 20988 \\
Dasysyrphus venustus & United States & North Carolina & JSS 20982 \\
Dasysyrphus venustus & United States & North Carolina & JSS 20807 \\
Dasysyrphus venustus & United States & Pennsylvania & USNM ENT 00258081 \\
Dasysyrphus venustus & United States & Pennsylvania & CNCD 5458 \\
Dasysyrphus venustus & United States & Utah & JSS 24642 \\
Dasysyrphus venustus & United States & Vermont & JSS 18338 \\
Dasysyrphus venustus & United States & Virginia & CNCD 5456 \\
Dasysyrphus venustus & United States & West Virginia & JSS 20692 \\
Eupeodes lapponicus & Canada & Manitoba & CHU05-FL-091 \\
Eupeodes lapponicus & Canada & Manitoba & JSS 18400 \\
Eupeodes sp. & Serbia & & JSS 19735 \\
Melangyna cincta & Germany & & CNCD 105572 \\
Melangyna guttata & Norway & CNCD 105574 \\
Melangyna lasiophthalma & Canada & Ontario & JSS 19748 \\
Melangyna sp. & South Korea & Kangwon-do & JSS 21045 \\
Melangyna triangulifera & Canada & British Columbia & CNCD 19312 \\
Scaeva dignota & Serbia & JSS 19737 \\
Scaeva pyrastri & United States & Colorado & JSS 20802
\end{tabular}


Table 2: Dasysyrphus mean and maximum intraspecific values and nearest neighbour values.

\begin{tabular}{|c|c|c|c|c|c|}
\hline Species & $\begin{array}{l}\text { Number of } \\
\text { Specimens }\end{array}$ & $\begin{array}{c}\text { Mean } \\
\text { Intraspecific } \\
\text { Variation (\%) } \\
\end{array}$ & $\begin{array}{c}\text { Maximum } \\
\text { Intraspecific } \\
\text { Variation (\%) }\end{array}$ & $\begin{array}{c}\text { Nearest } \\
\text { Neighbour (NN) }\end{array}$ & $\begin{array}{c}\text { Distance to } \\
\text { Nearest Neighbour } \\
(\%)\end{array}$ \\
\hline $\begin{array}{l}\text { D. Sp. } 19 \text { (from } \\
\text { Costa Rica) }\end{array}$ & 4 & 0.4 & 0.72 & $\begin{array}{l}\text { D. intrudens } \\
\text { complex }\end{array}$ & 5.46 \\
\hline $\begin{array}{l}\text { D. Sp. } 23 \text { (from } \\
\text { South Korea) }\end{array}$ & 1 & N/A & $\mathrm{N} / \mathrm{A}$ & D. pinastri & 0.16 \\
\hline D. albostriatus & 1 & $\mathrm{~N} / \mathrm{A}$ & $\mathrm{N} / \mathrm{A}$ & D. eggeri & 2.62 \\
\hline D. amalopis & 11 & 0.14 & 0.5 & D. lenensis & 0.31 \\
\hline D. bilineatus & 4 & 0.46 & 0.93 & $\begin{array}{l}\text { D. intrudens } \\
\text { complex }\end{array}$ & 5 \\
\hline D. creper & 22 & 0.31 & 0.87 & D. laticaudus & 1.29 \\
\hline D. eggeri & 1 & $N / A$ & $\mathrm{~N} / \mathrm{A}$ & D. albostriatus & 2.62 \\
\hline $\begin{array}{l}\text { D. intrudens } \\
\text { complex }\end{array}$ & 135 & 1.92 & 4.79 & D. amalopis & 3.24 \\
\hline D. laticaudus & 21 & 0.17 & 1.24 & D. pauxillus & 0.46 \\
\hline D. lenensis & 1 & $\mathrm{~N} / \mathrm{A}$ & $\mathrm{N} / \mathrm{A}$ & D. amalopis & 0.31 \\
\hline D. limatus & 6 & 0.25 & 0.62 & D. tricinctus & 0.46 \\
\hline D. lotus & 3 & 3.09 & 4.30 & D. amalopis & 3.61 \\
\hline D. nigricornis & 7 & 0.31 & 0.94 & D. occidualis & 0 \\
\hline D. occidualis & 12 & 0.11 & 0.32 & D. nigricornis & 0 \\
\hline D. pacificus & 22 & 0.43 & 1.18 & D. laticaudus & 1.4 \\
\hline D. pauxillus & 18 & 0.1 & 0.54 & D. laticaudus & 0.46 \\
\hline D. pinastri & 2 & 0 & 0 & $\begin{array}{l}\text { D. Sp. } 23 \text { (from } \\
\text { South Korea) }\end{array}$ & 0.16 \\
\hline D. tricinctus & 3 & 0 & 0 & D. limatus & 0.46 \\
\hline D. venustus & 70 & 0.82 & 3.15 & $\begin{array}{l}\text { D. intrudens } \\
\text { complex }\end{array}$ & 3.62 \\
\hline
\end{tabular}




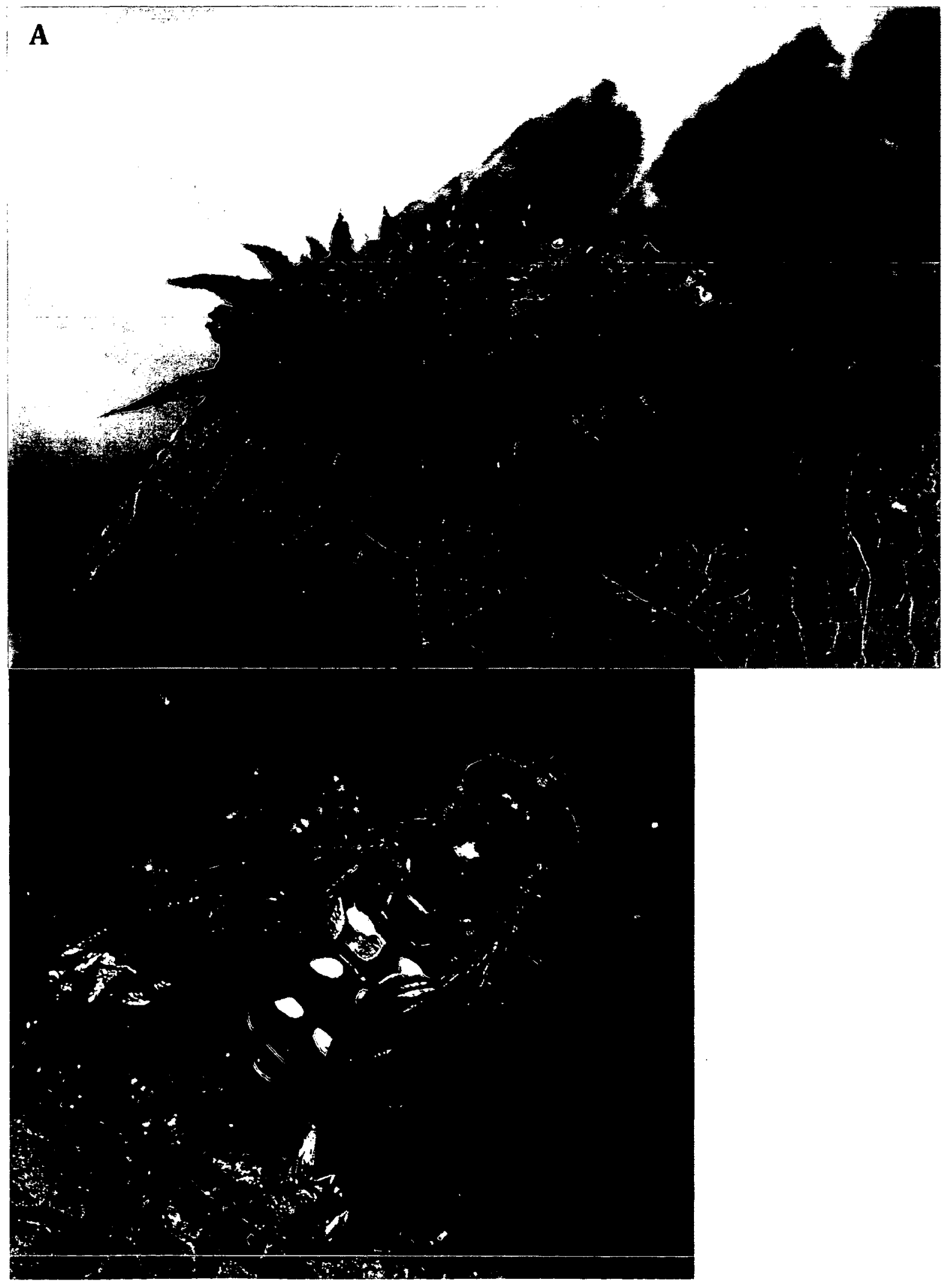

Fig. 1: A: Dasysyrphus sp. larva (Russia; courtsey of Vladimir Davydov); B: Dasysyrphus intrudens complex adult female (Washington, USA; courtsey of Andrew Young). 


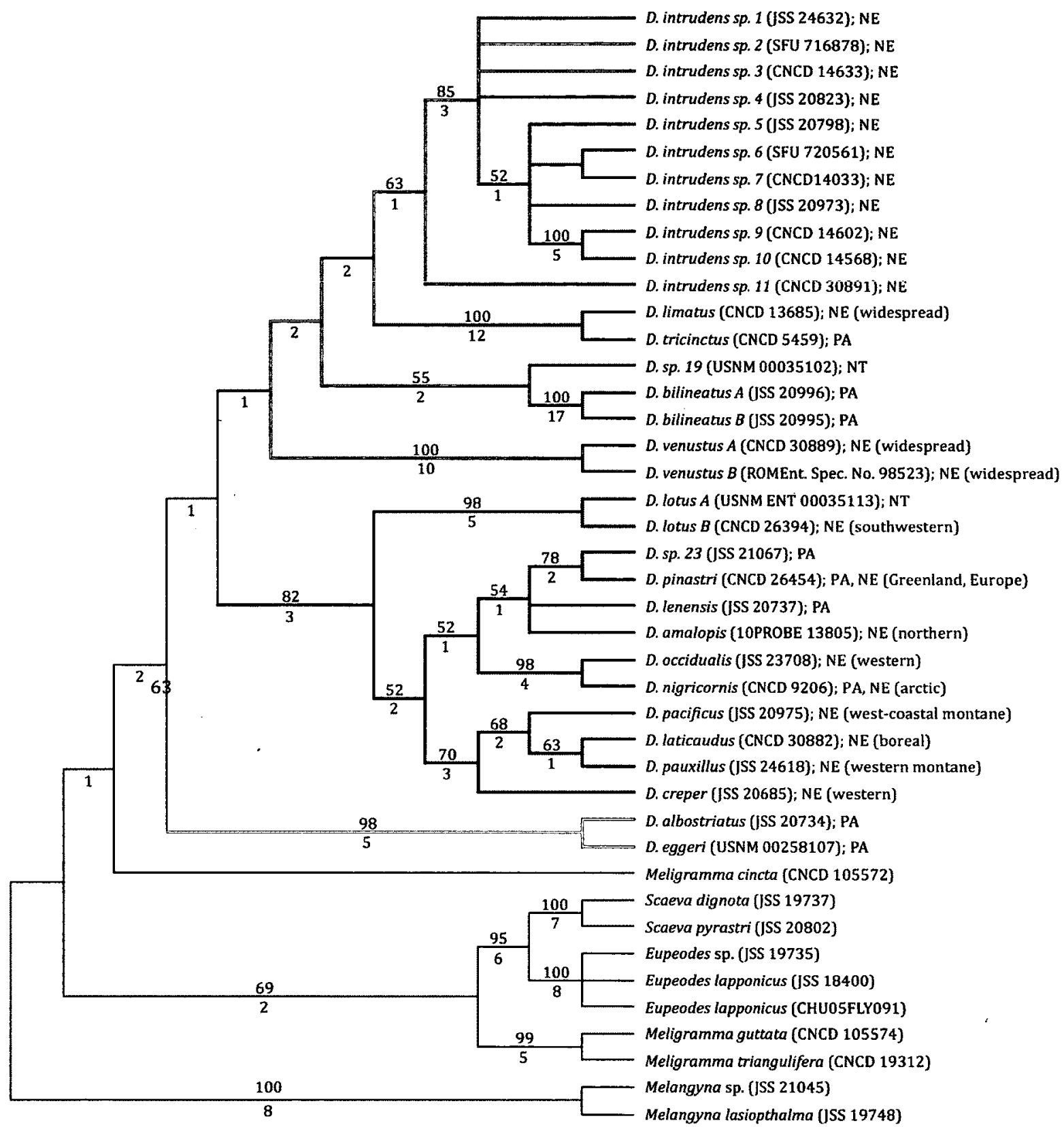

Fig. 2: Semi-strict consensus tree from 26 most parsimonious trees (Consistency Index $(\mathrm{CI})$ excluding uninformative characters $=0.4141$, Retention Index $(\mathrm{RI})=$ 0.6826 ); showing Jackknife support values above and Bremer support values below. Species groups are highlighted as follows: albostriarus group: orange, pinastri group: blue, tricinctus group: green and venustus group: red (follows updated species groups). 


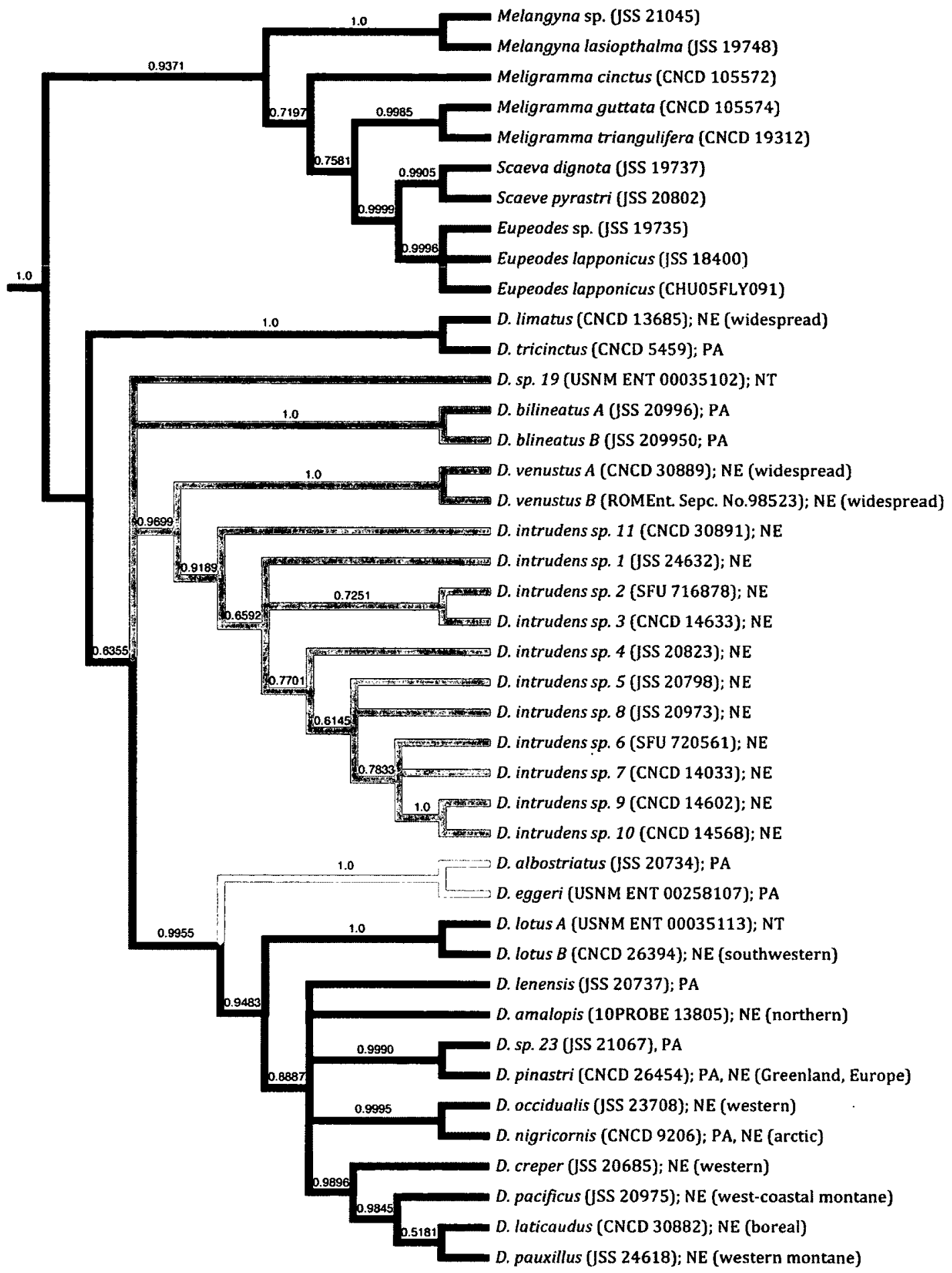

Fig. 3:Majority rule consensus tree from 8002 trees of Bayesian Markov Chain Monte Carlo analysis. Species groups are highlighted as follows: albostriarus group: orange, pinastri group: blue, tricinctus group: green and venustus group: red (follows updated species groups). 


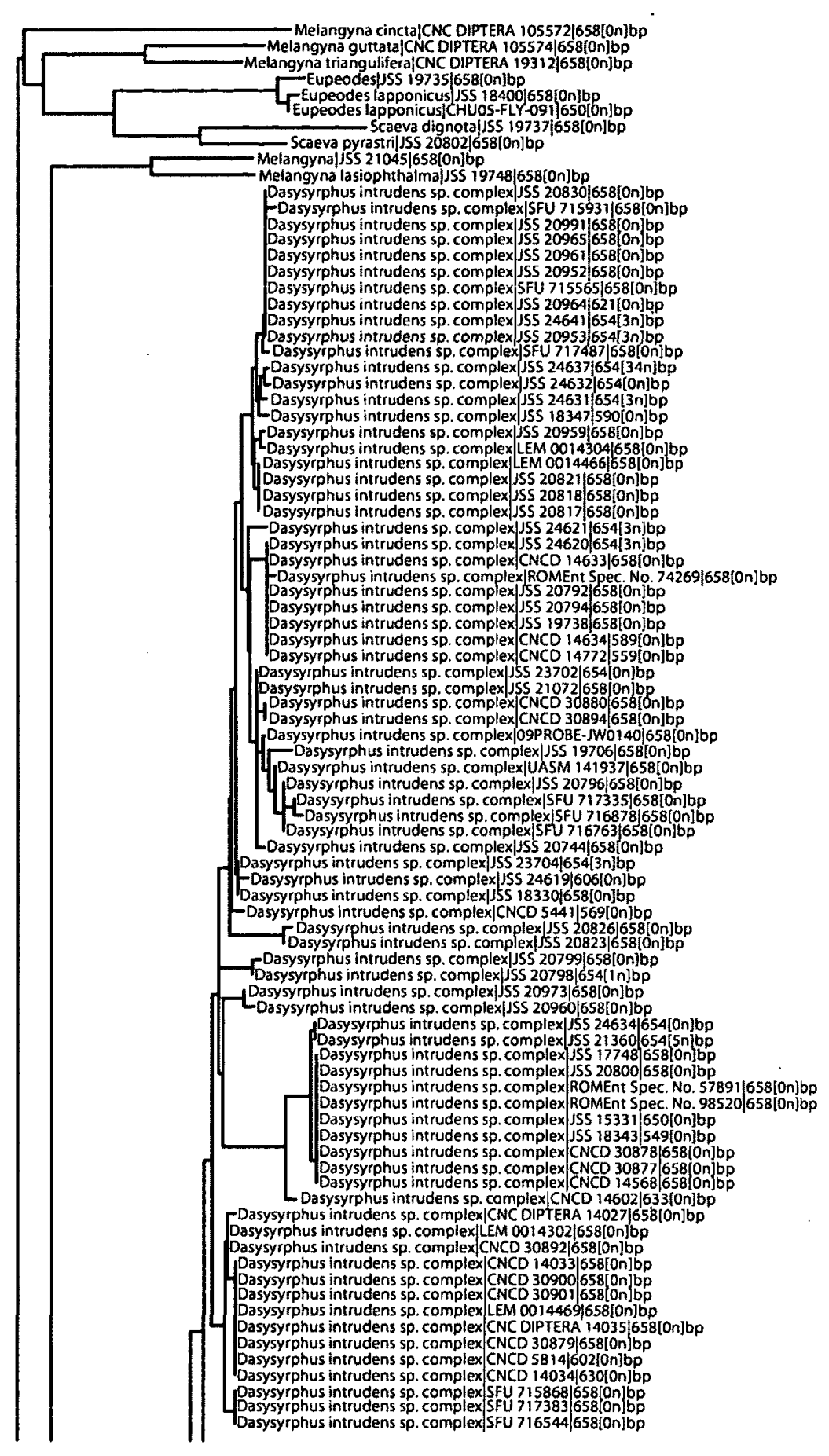

Fig. 4: Neighbour-joining tree of $354 \mathrm{COI}$ sequences. 


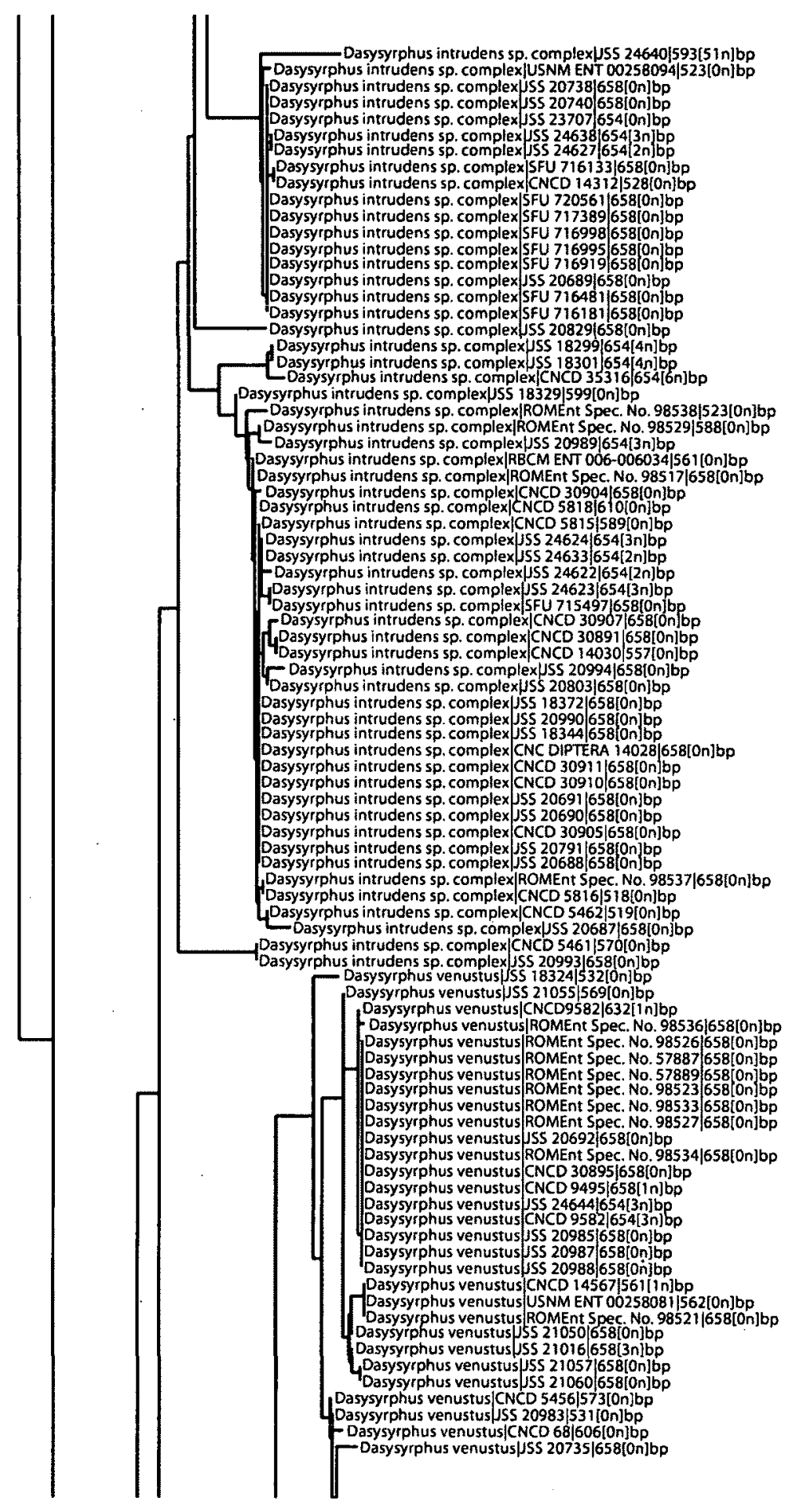

Fig. 4: continued. 


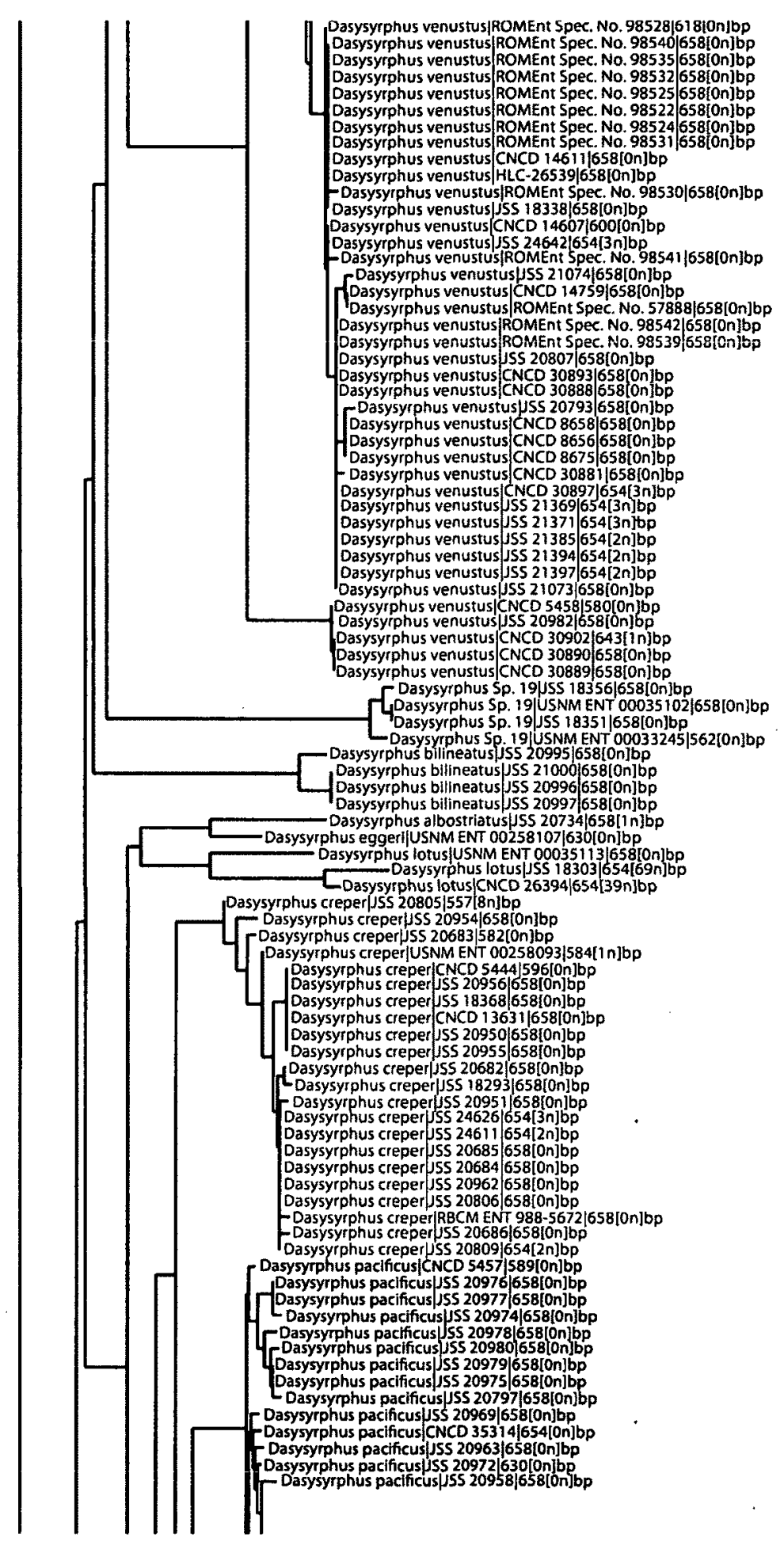

Fig. 4: continued. 


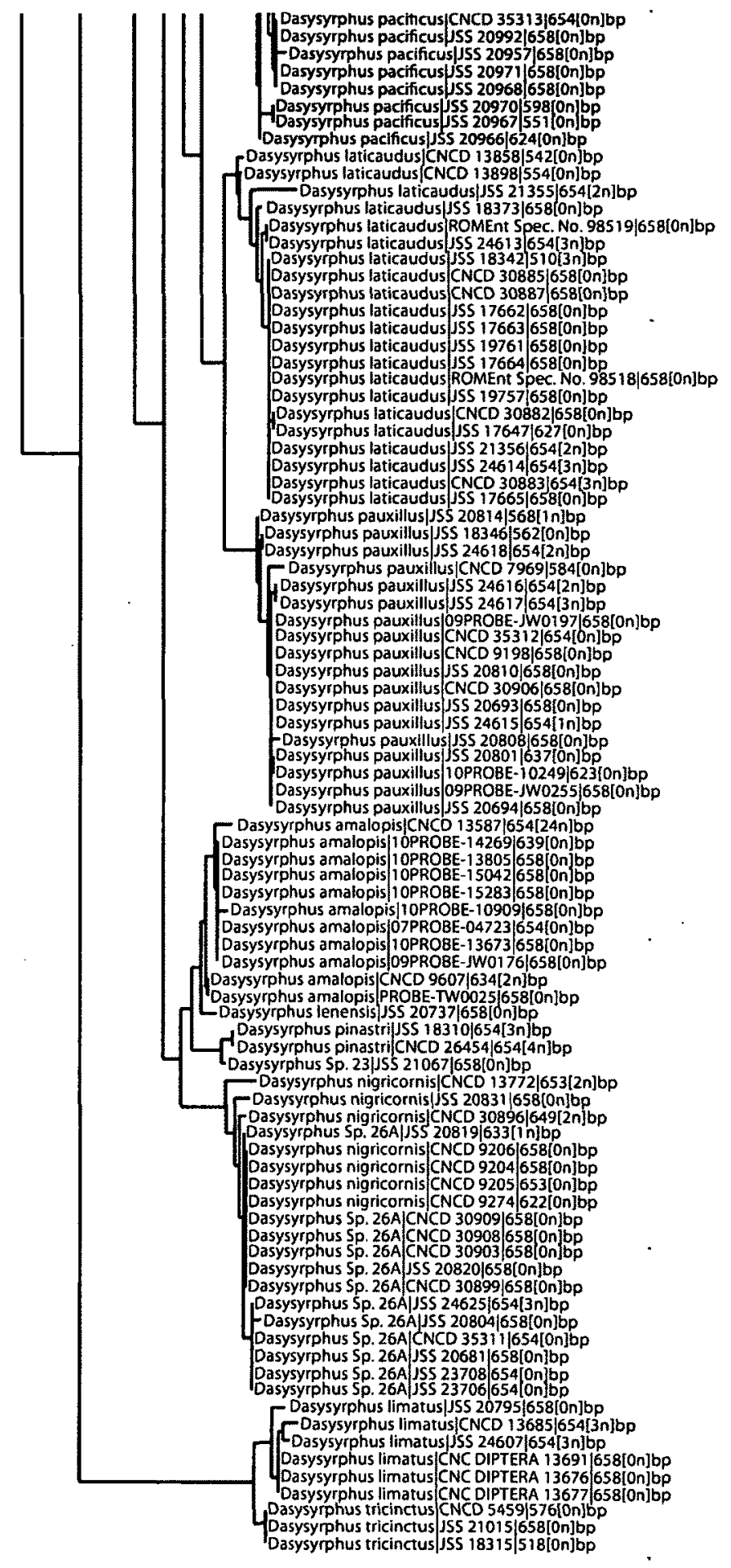

Fig. 4: continued. 

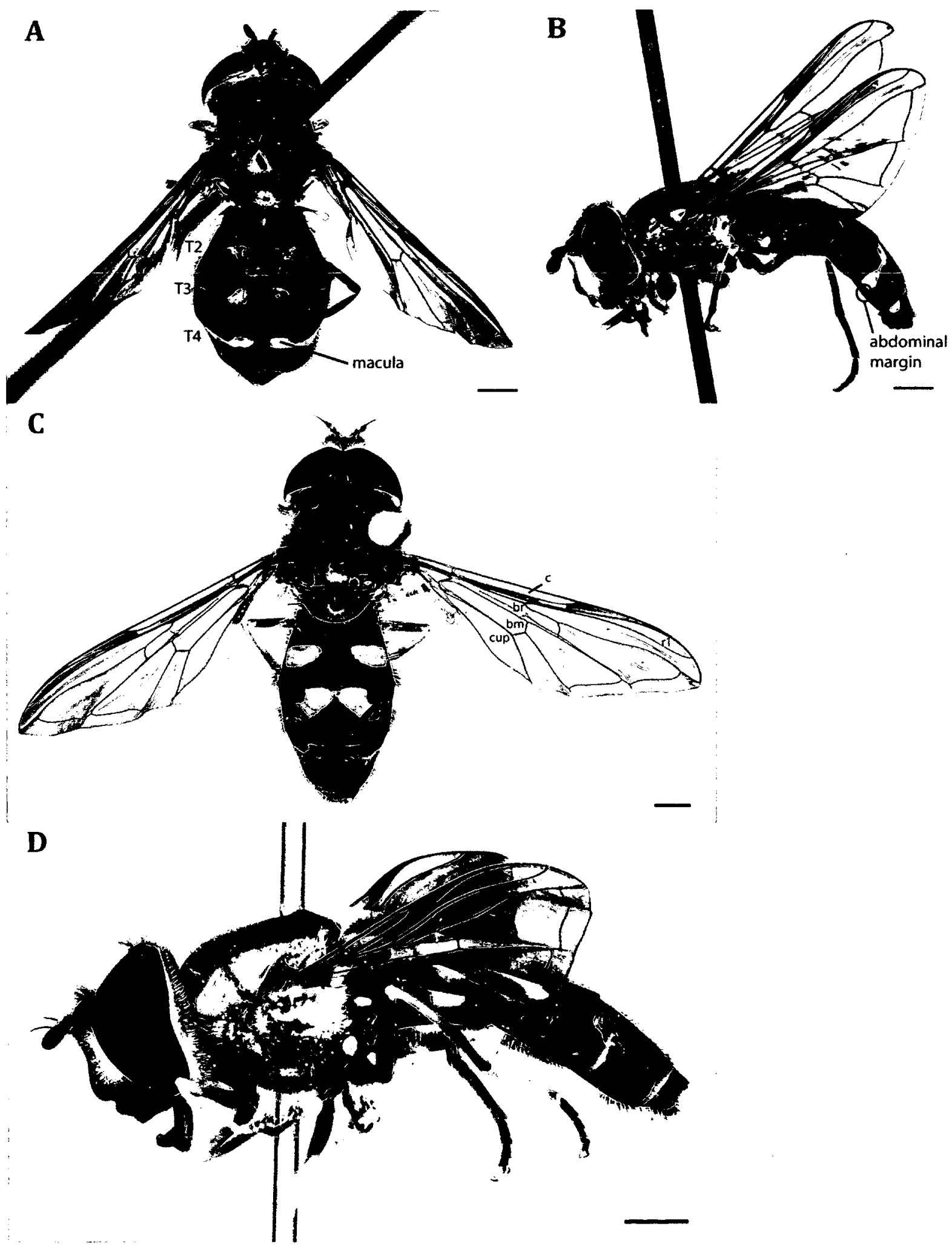

Fig. 5: A: Dasysyrphus amalopis (CNCD 13616) female dorsal habitus; B: D. amalopis (CNCD 13616) female lateral habitus; C: D. creper (CNCD 26355) male dorsal habitus; D: D. creper (CNCD 26355) male lateral habitus, genitalia removed. $\mathrm{T}=$ tergite. Scale bar $=1 \mathrm{~mm}$. 


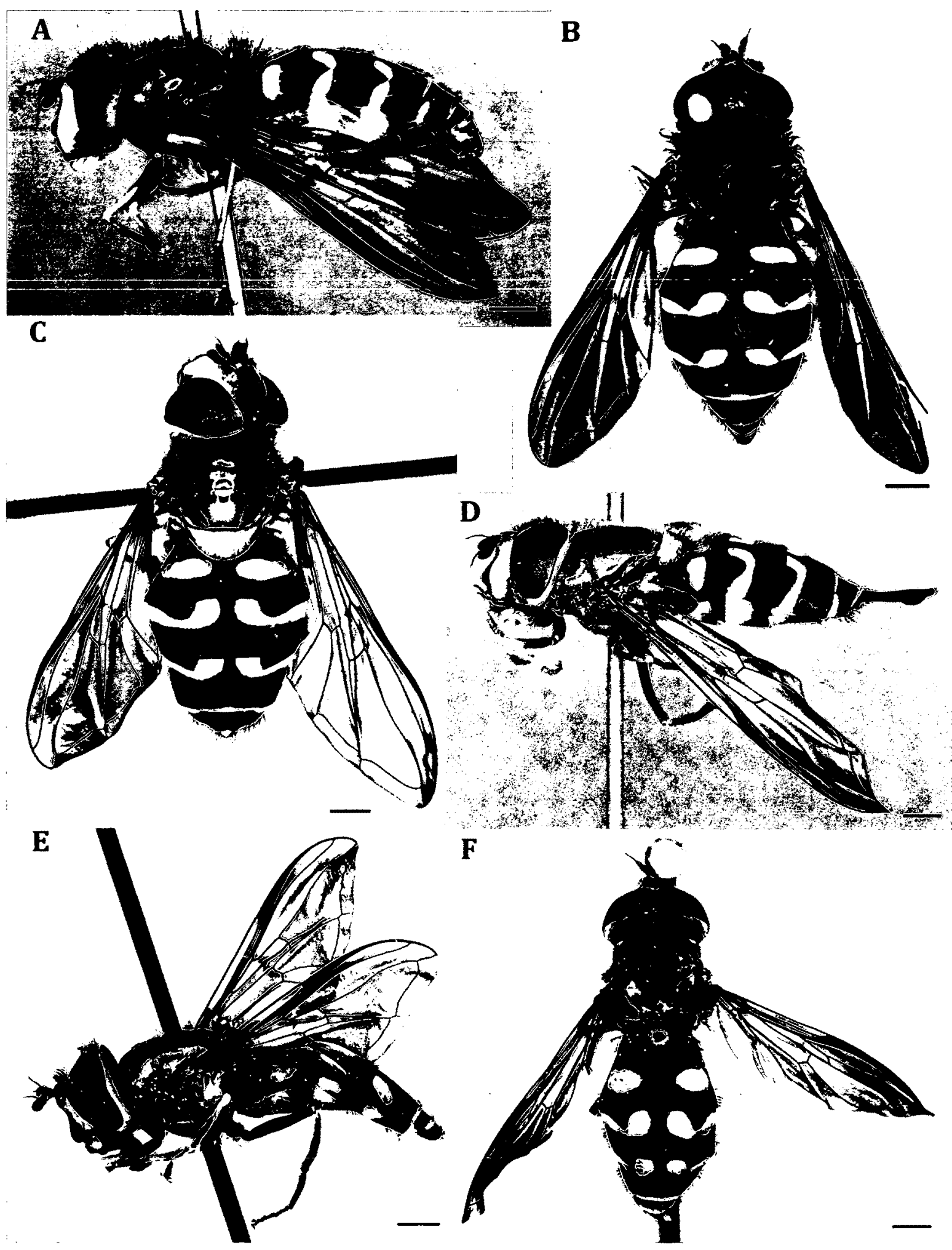

Fig: 6: Dasysyrphus intrudens complex: A: (CNCD 14312) female lateral habitus; B: (CNCD 14312) female dorsal habitus, C: (CNCD 30911) female dorsal habitus, D: (CNCD 30911) female lateral habitus, E: (JSS 23704) male lateral habitus, F: (JSS 23704) male dorsal habitus. Scale bar $=1 \mathrm{~mm}$. 


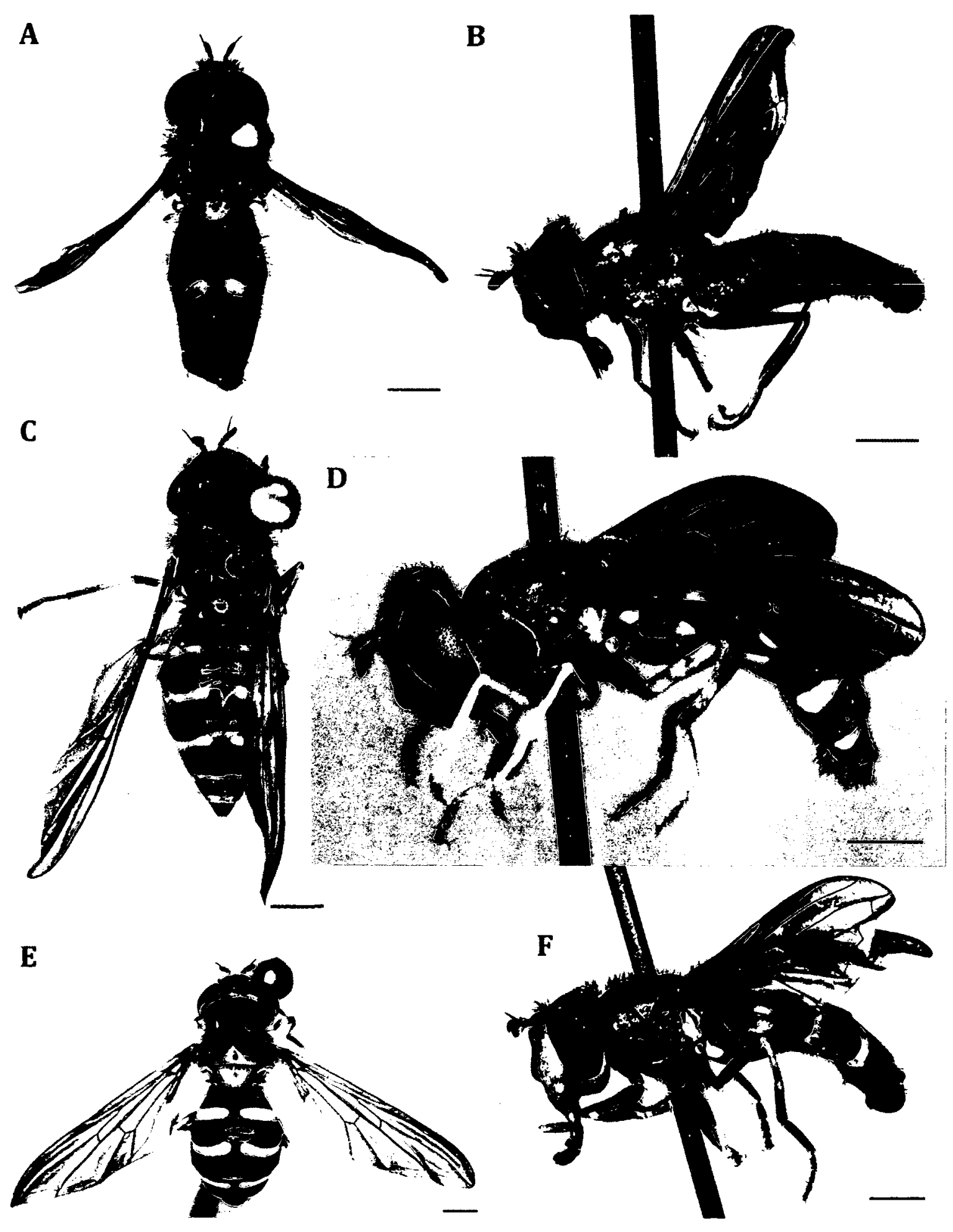

Fig. 7: A: Dasysyrphus nigricornis (CNCD 13711) male dorsal habitus; B: D. nigricornis (CNCD 13711) male lateral habitus; C: D. nigricornis (CNCD 13568) female dorsal habitus; D: D. laticaudus (CNCD 13875) female lateral habitus; E: D. laticaudus (CNCD 13875) female dorsal habitus; F: (JSS 20763) male lateral habitus. Scale bar $=1 \mathrm{~mm}$. 


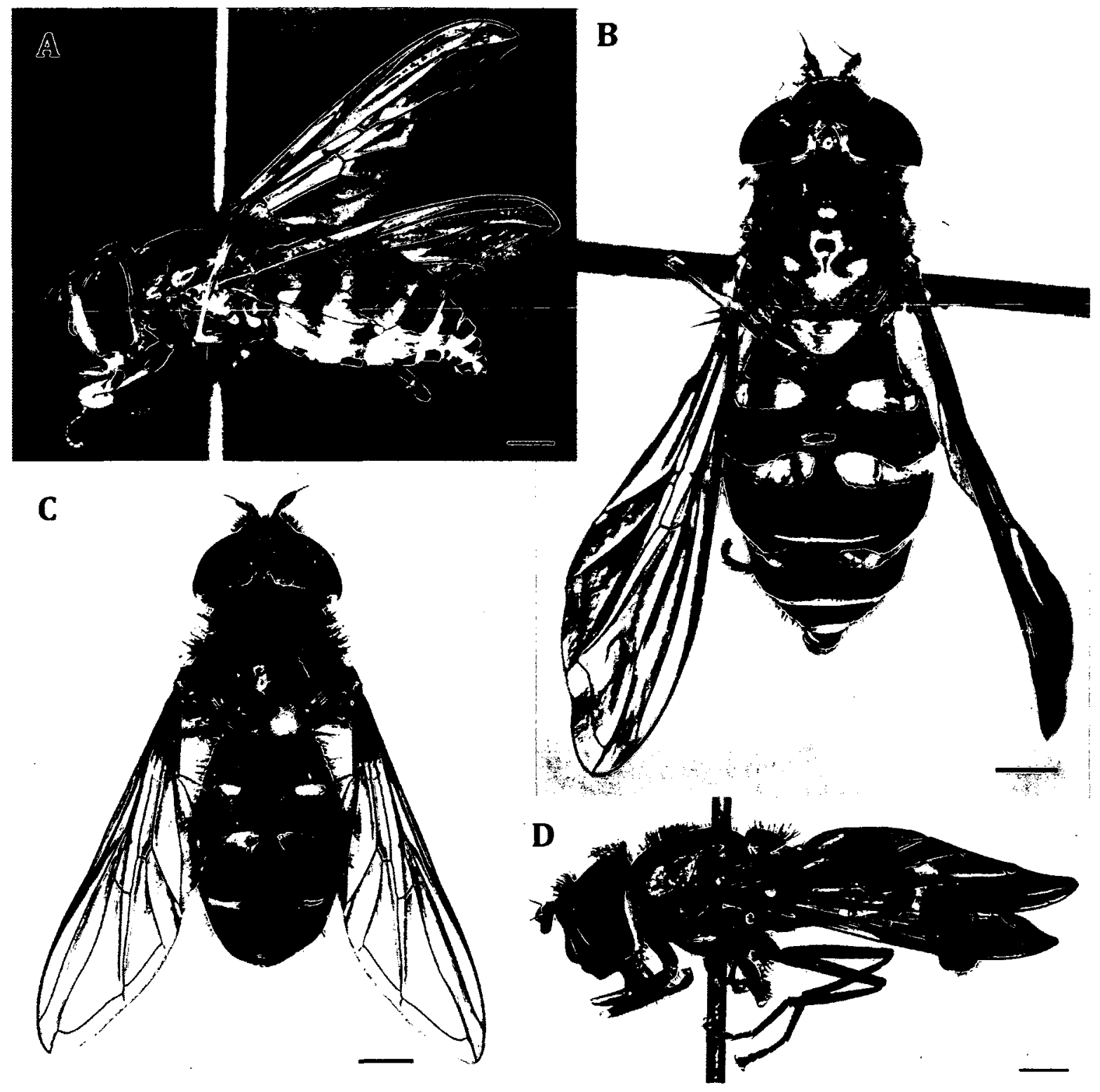

Fig. 8: A: Dasysyrphus venustus (CNCD 30888) fenake lateral habitus; $B$ : $D$. venustus (CNCD 30888) female dorsal habitus; C: D. limatus (CNCD 13659) male dorsl habitus; D: D. limatus (CNCD 13659) male lateral habitus. Scale bar $=1 \mathrm{~mm}$. 


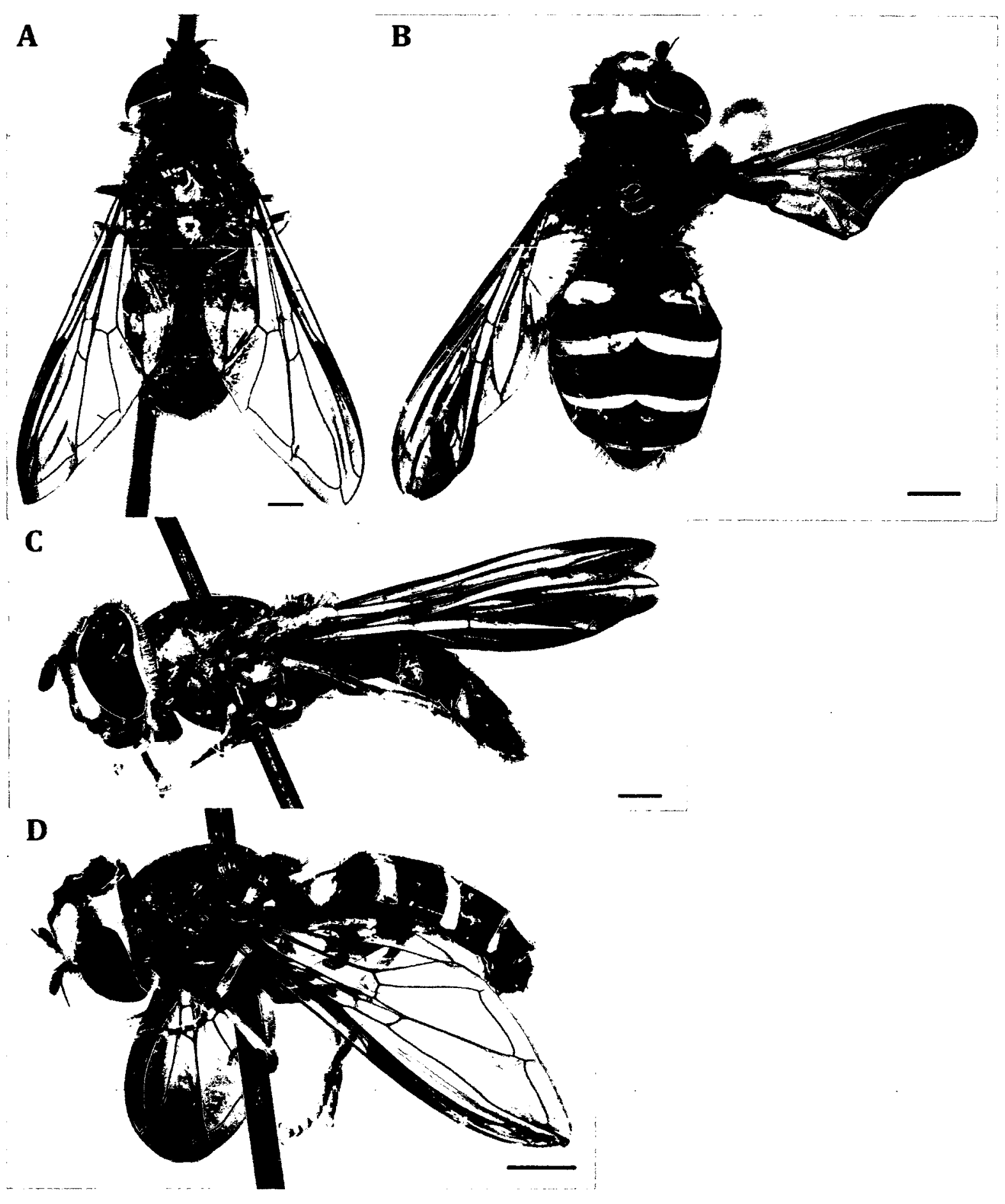

Fig. 9: A: Dasysyrphus lotus (CNCD 26389) female dorsal habitus; B: D. richardi (USNM ENT 00258072) female dorsal habitus; C: D. lotus (CNCD 26389) female lateral habitus; D: D. richardi (USNM ENT 00258072) lateral habitus. Scale bar = $1 \mathrm{~mm}$. 


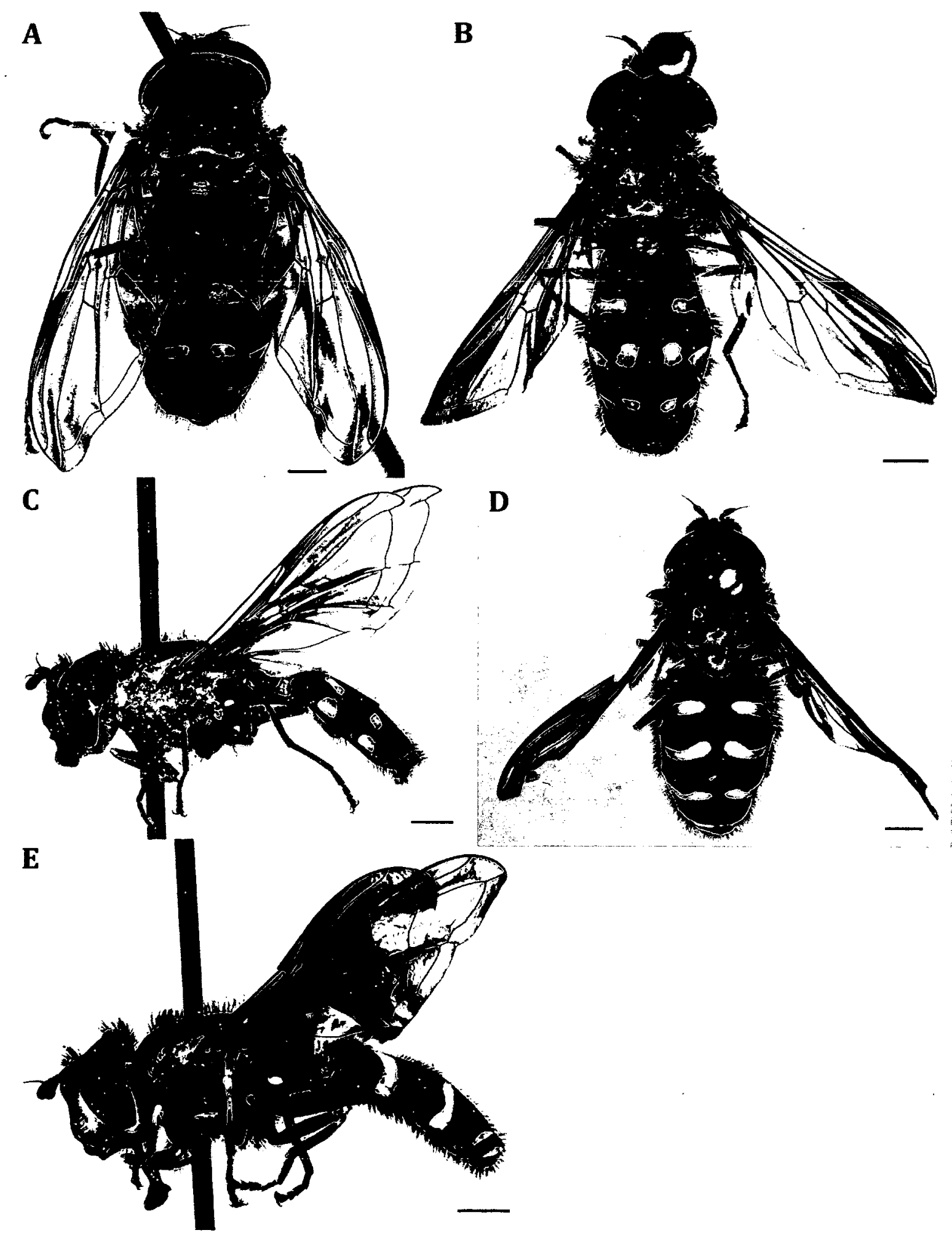

Fig. 10: A: Dasysyrphus occidualis (CNCD 60043) female dorsal habitus; B: D. occidualis (OSU 0000133386) male dorsal habitus; C: D. occidualis (OSU 0000133386) male lateral habitus; D: D. pacificus (CNCD 35314) male dorsal habitus; E: D. pacificus (CNCD 35314) male lateral habitus. Scale bar $=1 \mathrm{~mm}$. 


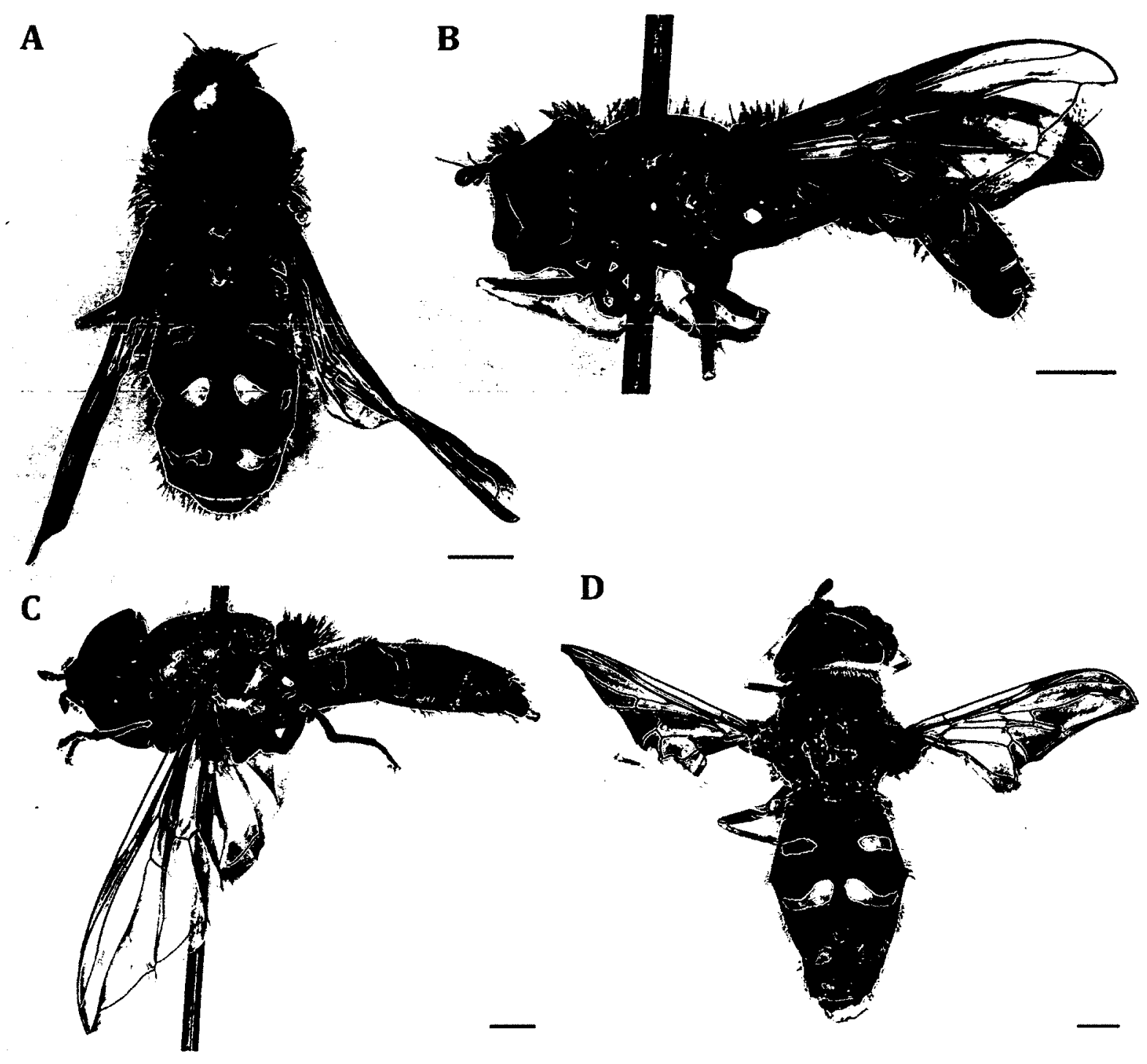

Fig. 11: A: Dasysyrphus pauxillus (JSS 24616) male dorsal habitus; B: D. pauxillus (JSS 24616) male lateral habitus; C: D. pinastri (JSS 18310) male lateral habitus (genitalia removed); D: D. pinastri (JSS 18310) dorsal habitus (genitalia removed). Sacle bar $=1 \mathrm{~mm}$. 
A

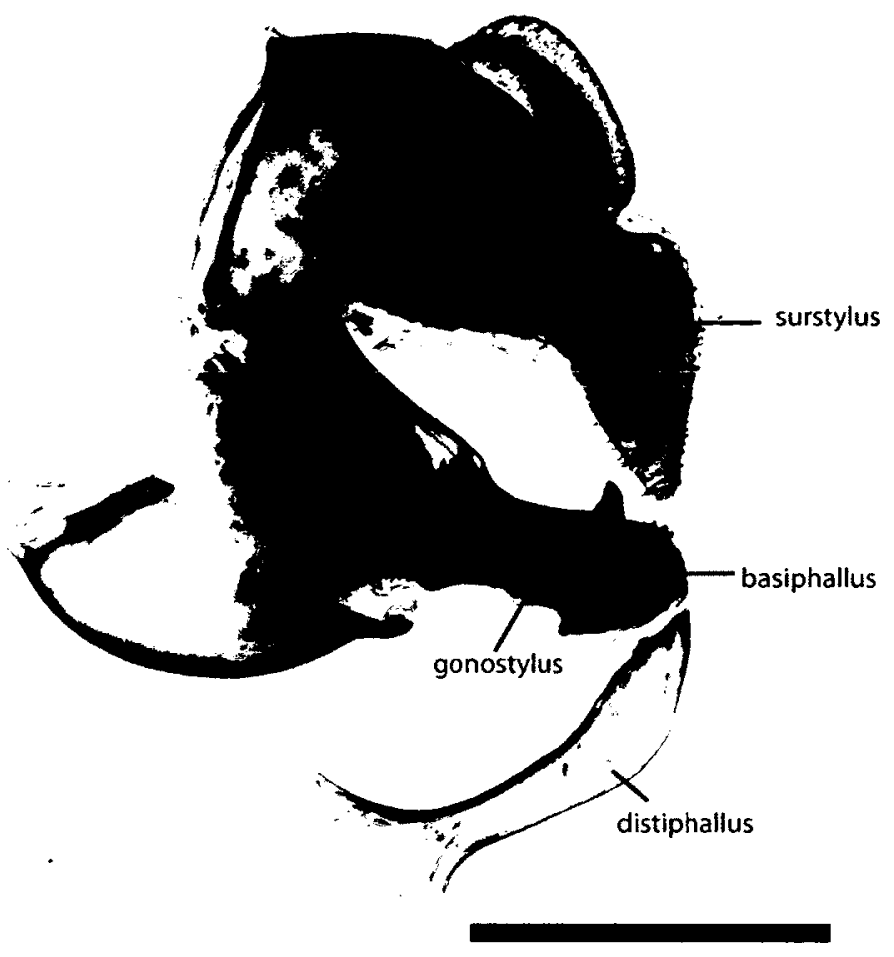

B

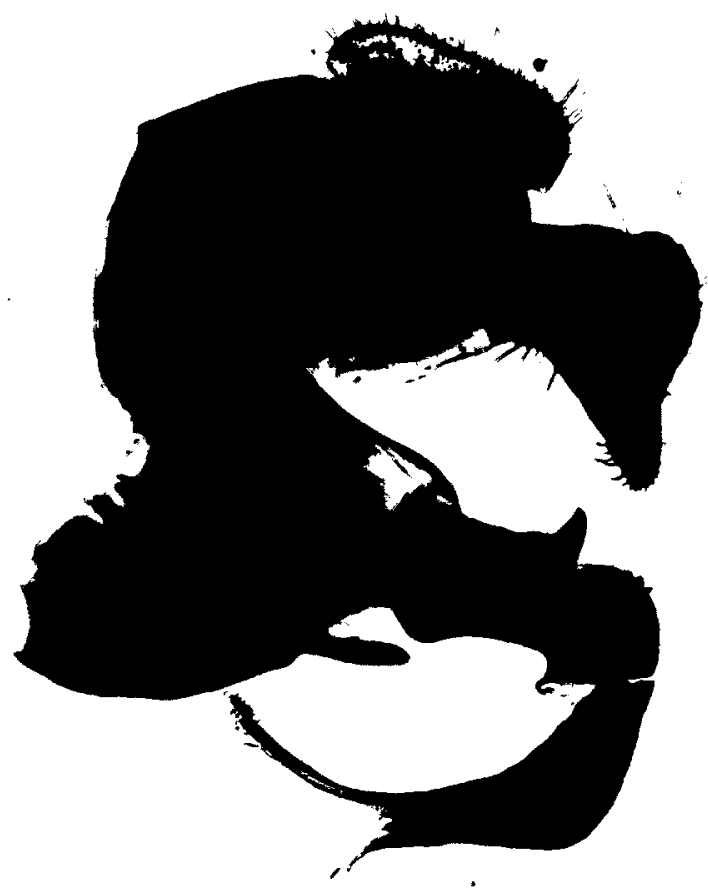

Fig 12: A: Dasysyrphus amalopis (CNCD 9607) lateral genitalia; B: D. occidualis (JSS 20804) lateral genitalia. Scale bar $=500 \mu \mathrm{m}$. 
A

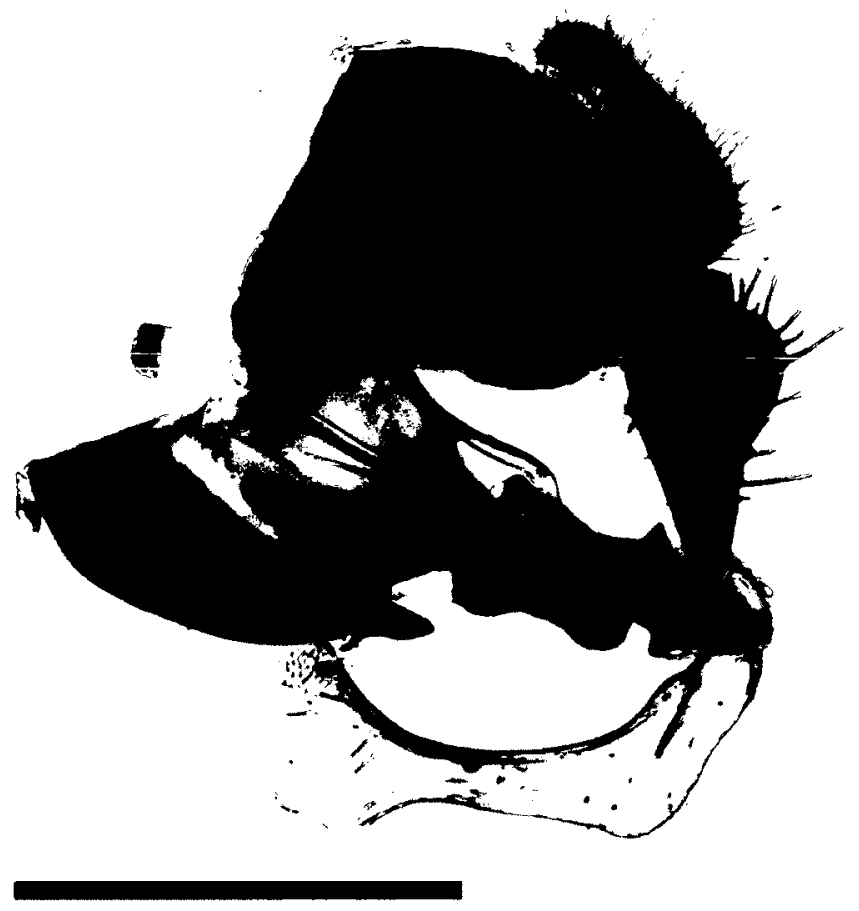

B

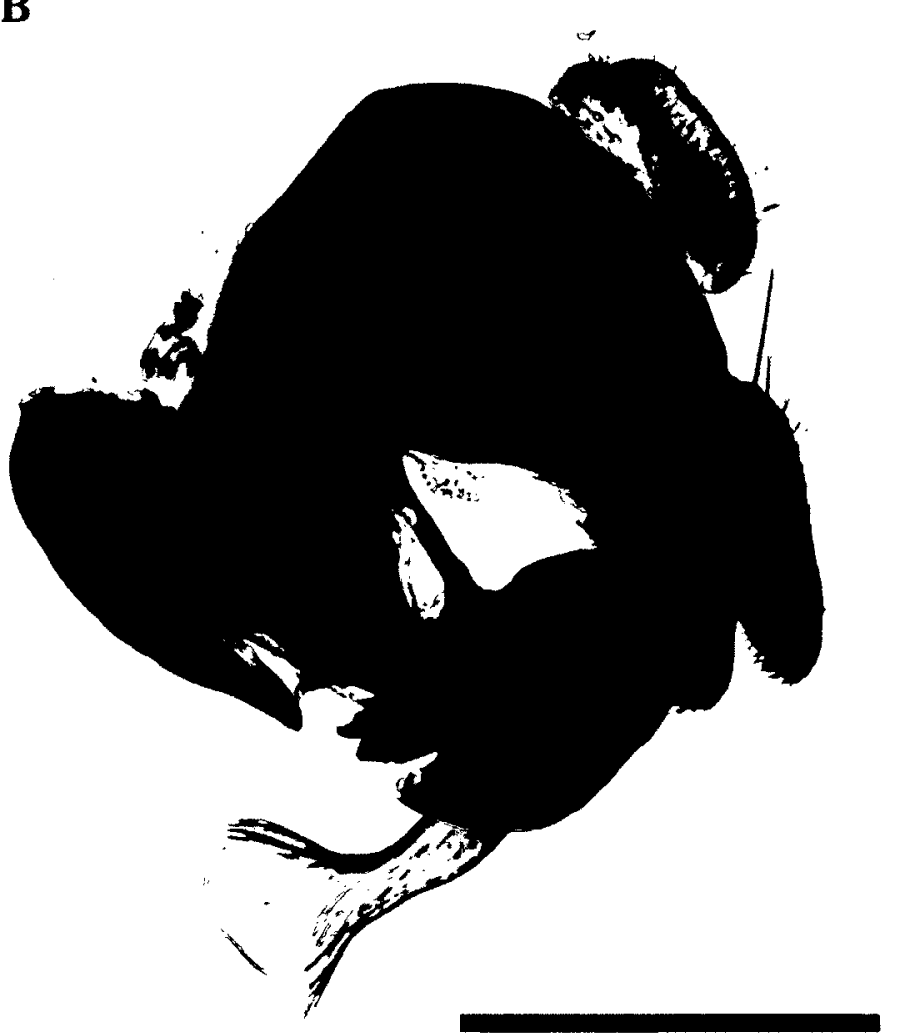

Fig 13: A: Dasysyrphus creper (JSS 20682) lateral genitalia; B: D. limatus (JSS 20796) lateral genitalia. Scale bar $=500 \mu \mathrm{m}$. 

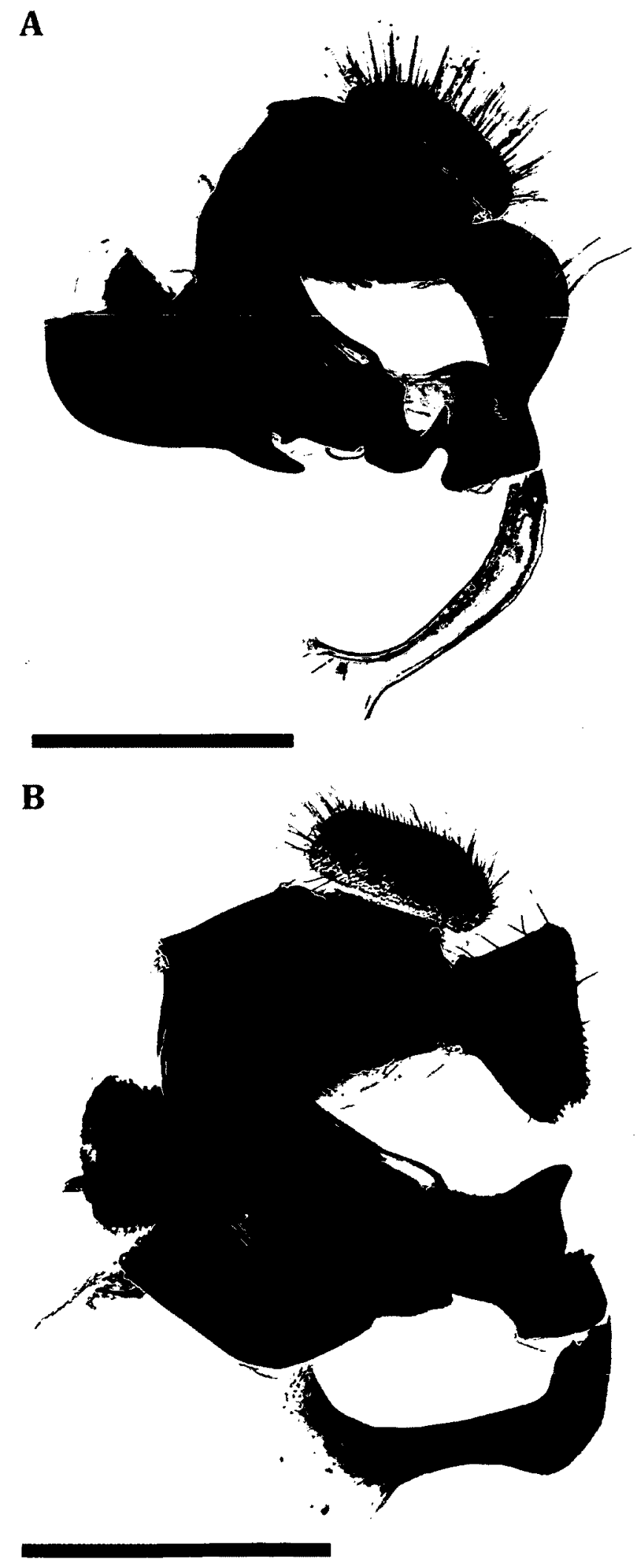

Fig 14: A: Dasysyrphus lotus (JSS 18303) lateral genitalia; B: D. nigricornis (CNCD 9206) lateral genitalia. Scale bar $=500 \mu \mathrm{m}$. 
A
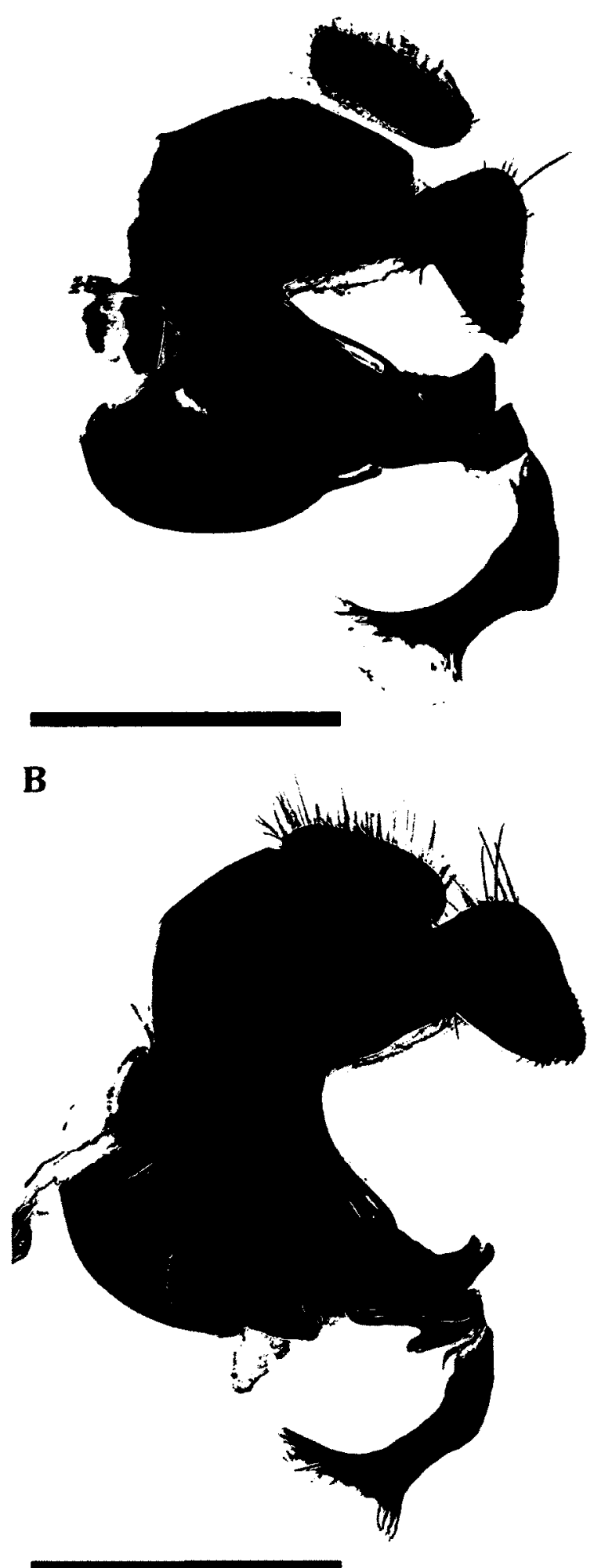

Fig 15: A: Dasysyrphus laticaudus (CNCD 30087) lateral genitalia; B: D. pacificus (CNCD 26435) lateral genitalia. Scale bar $=500 \mu \mathrm{m}$. 
A
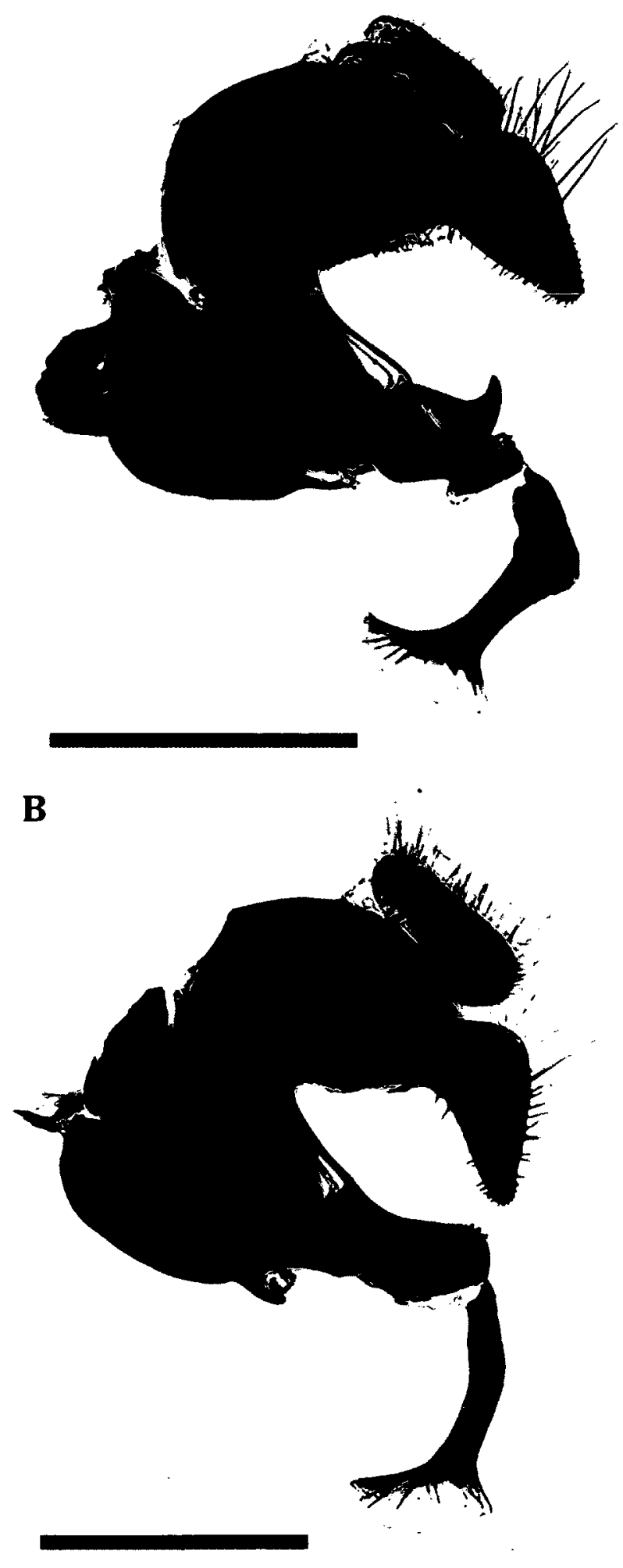

Fig 16: A: Dasysyrphus pauxillus (CNCD 13746) lateral genitalia; B: D. pinastri (JSS 18310) lateral genitalia. Scale $b a r=500 \mu \mathrm{m}$. 


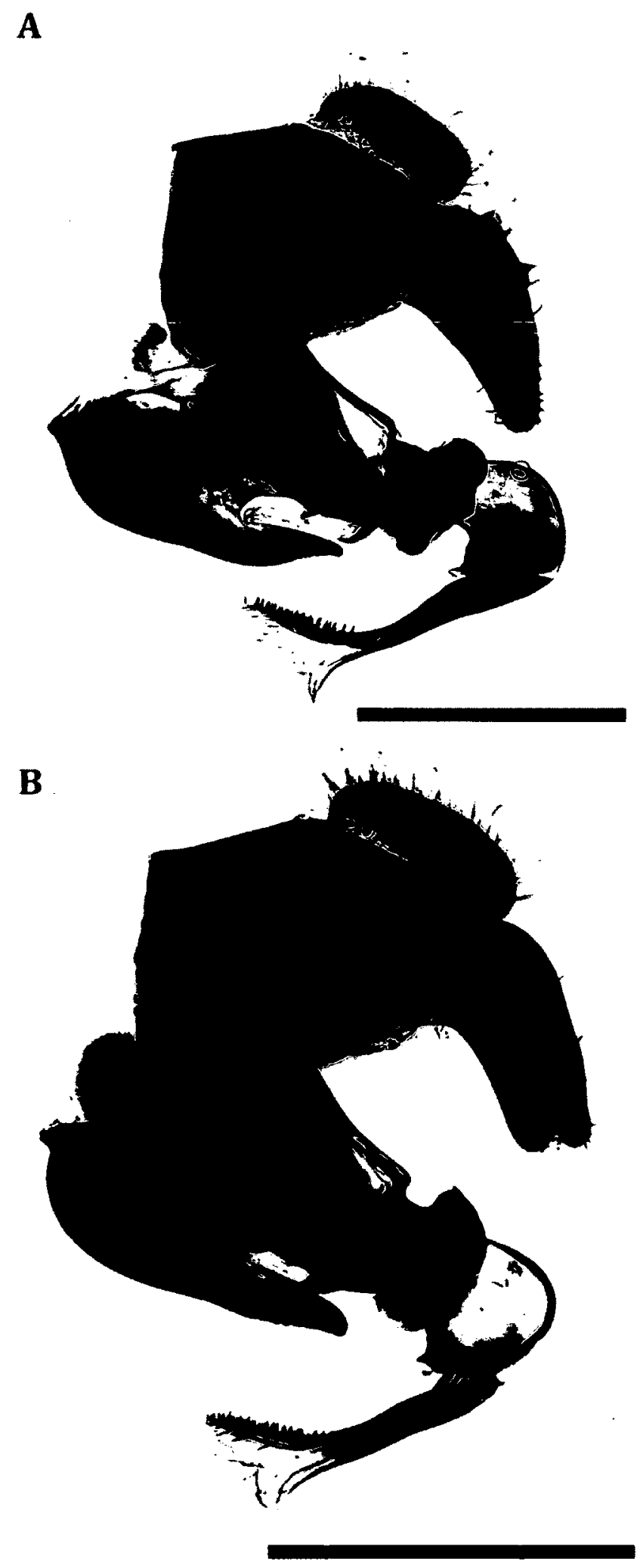

Fig 17: A: Dasysyrphus intrudens complex (CNCD 30894) lateral genitalia; B: $D$. venustus (CNCD 14567) lateral genitalia. Scale bar $=500 \mu \mathrm{m}$. 


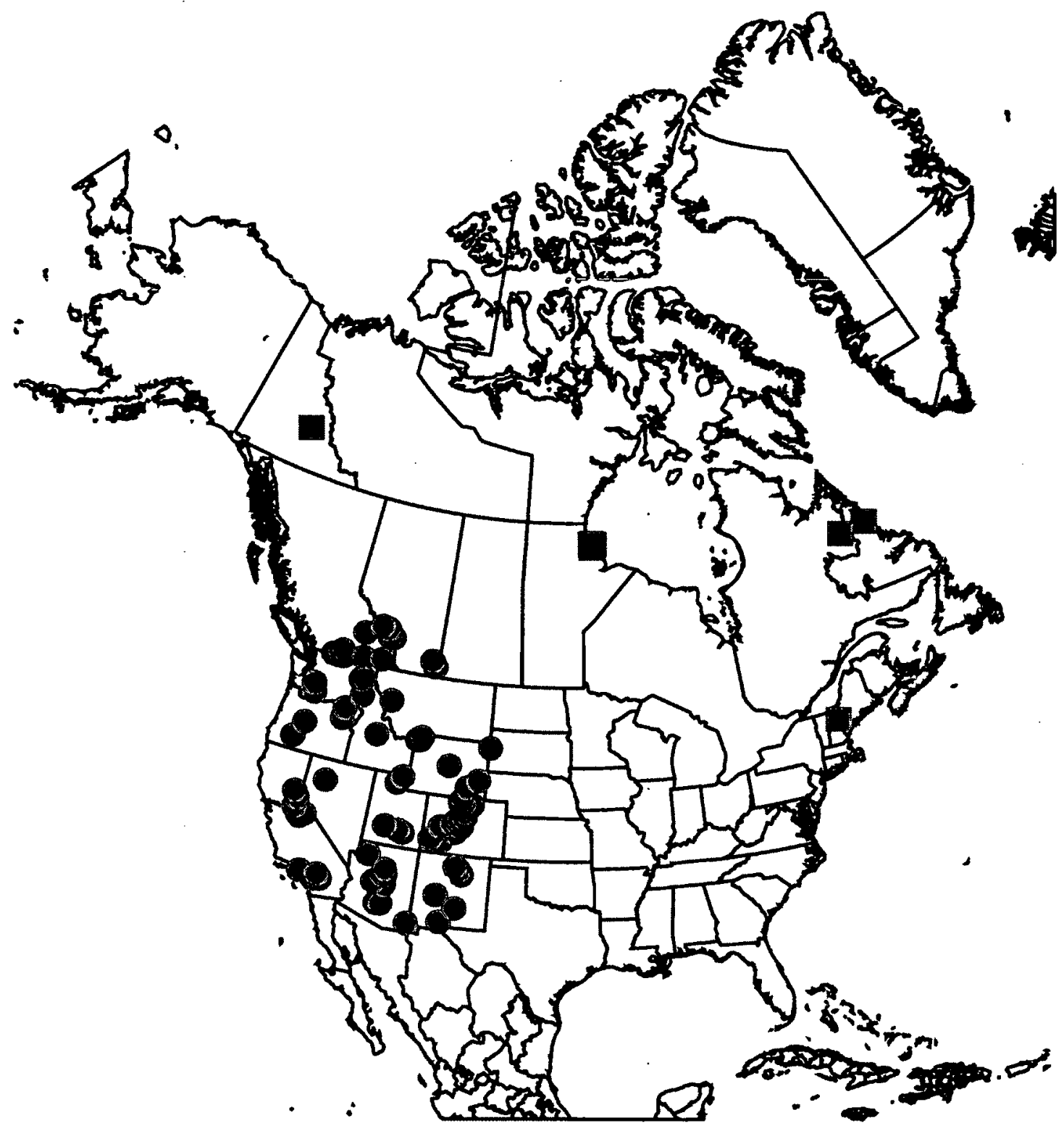

Fig. 18: Dasysyrphus amalopis (squares) and D. creper (circles) range map (symbols indicate specimen point data). 


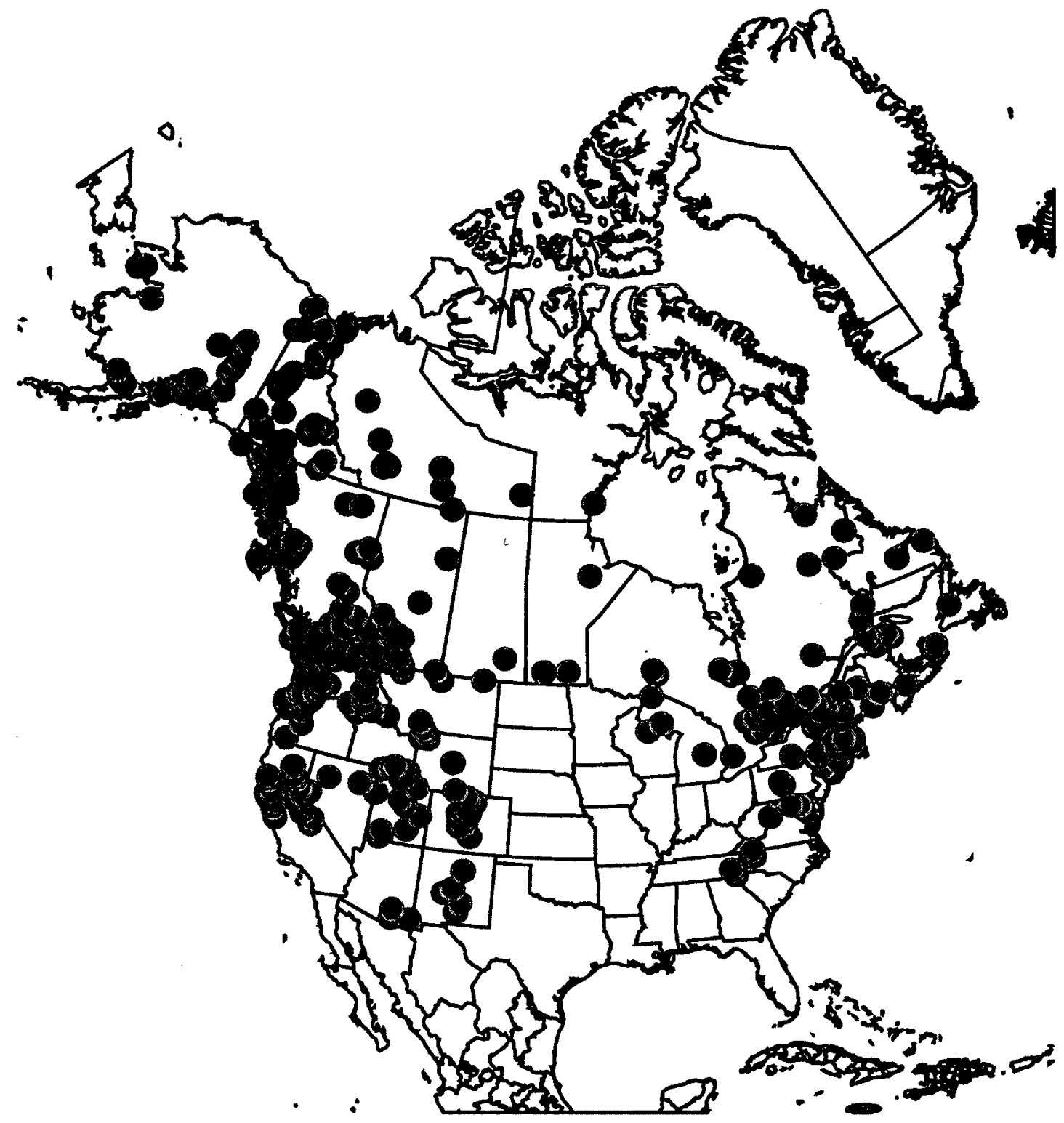

Fig. 19: Dasysyrphus intrudens complex range map (circles indicate specimen point data). 


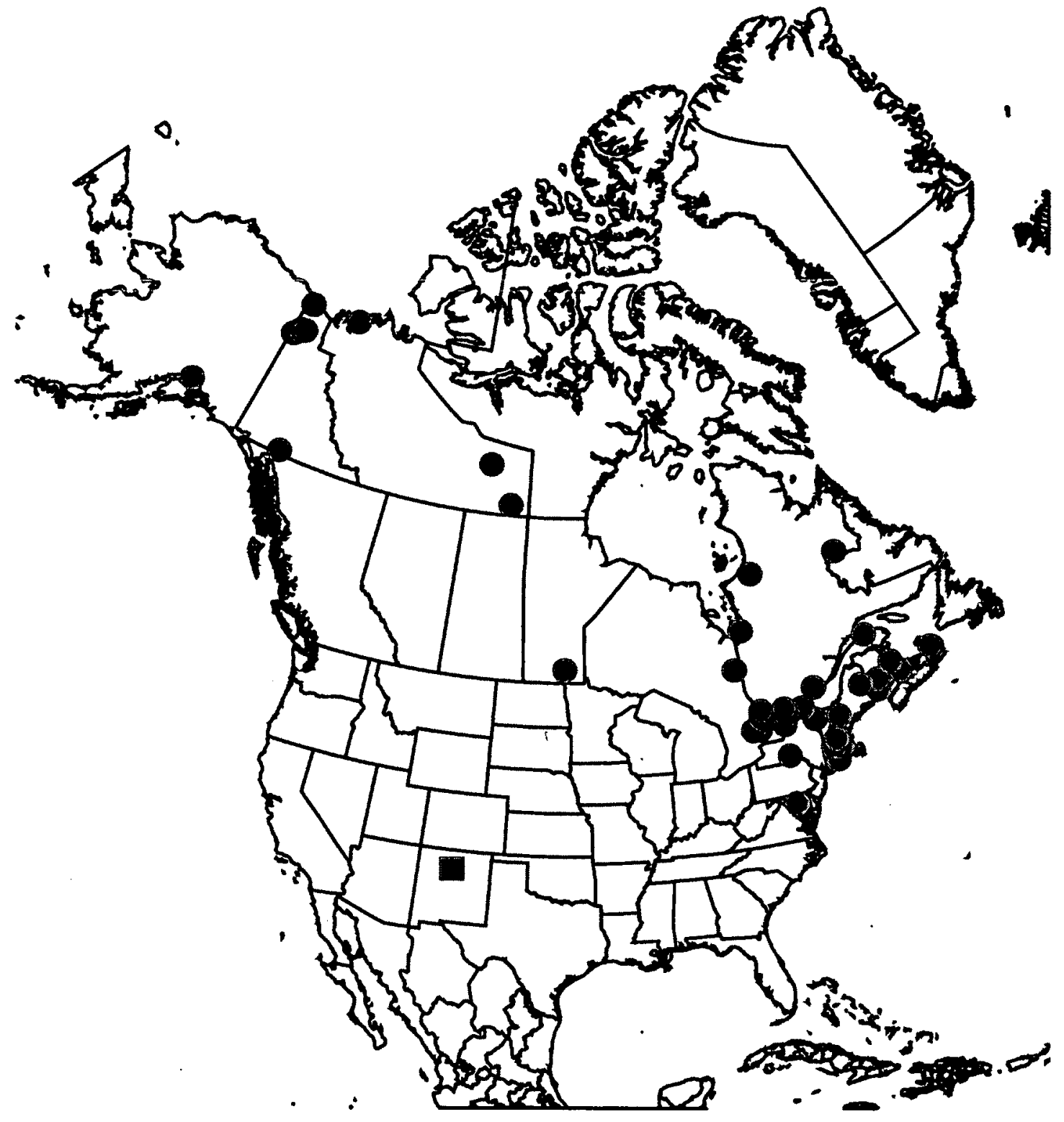

Fig. 20: Dasysyrphus laticaudus (circles) and D. richardi (squares) range map (symbols indicate specimen point data). 


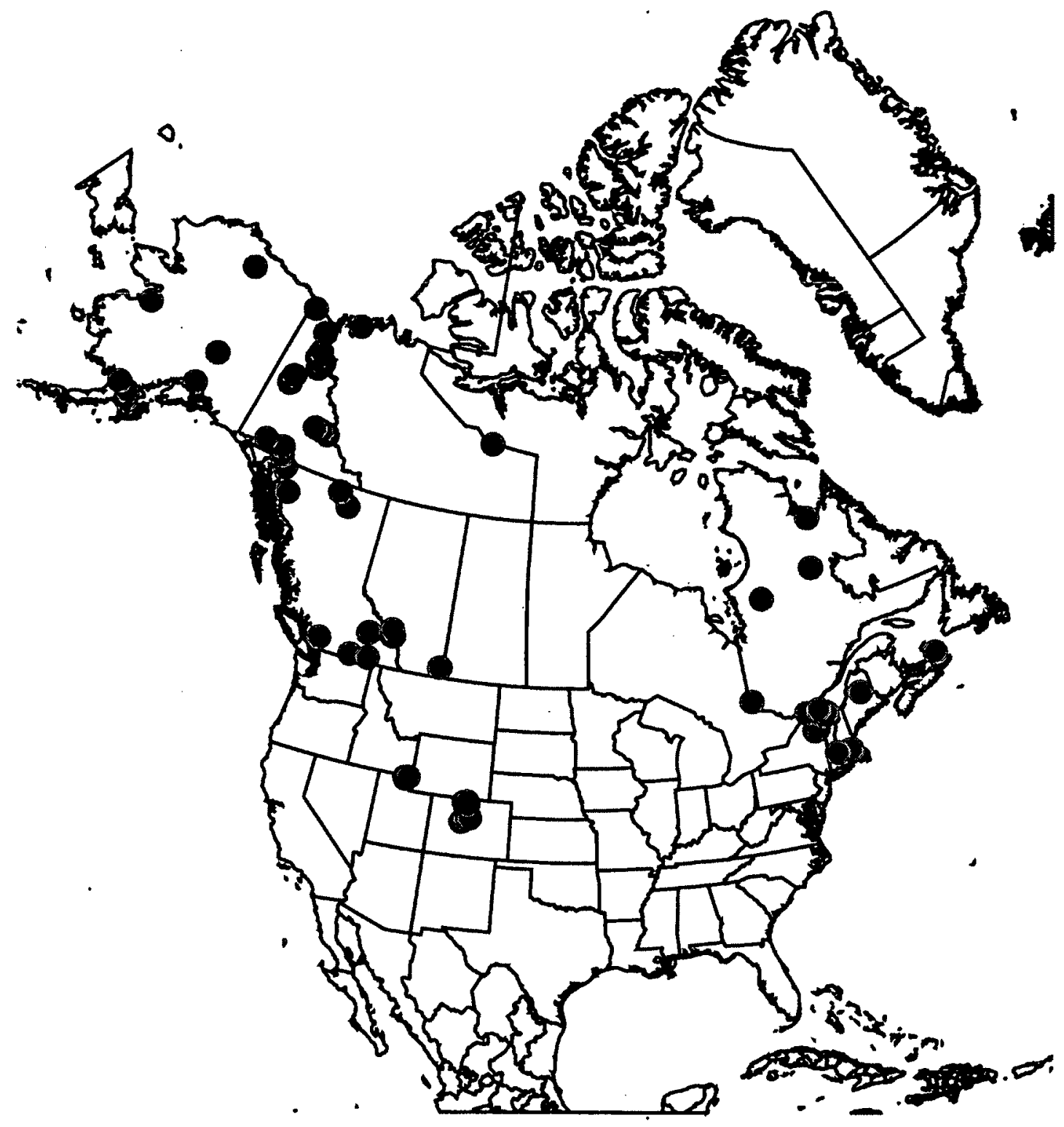

Fig. 21: Dasysyrphus limatus range map (circles indicate specimen point data) 


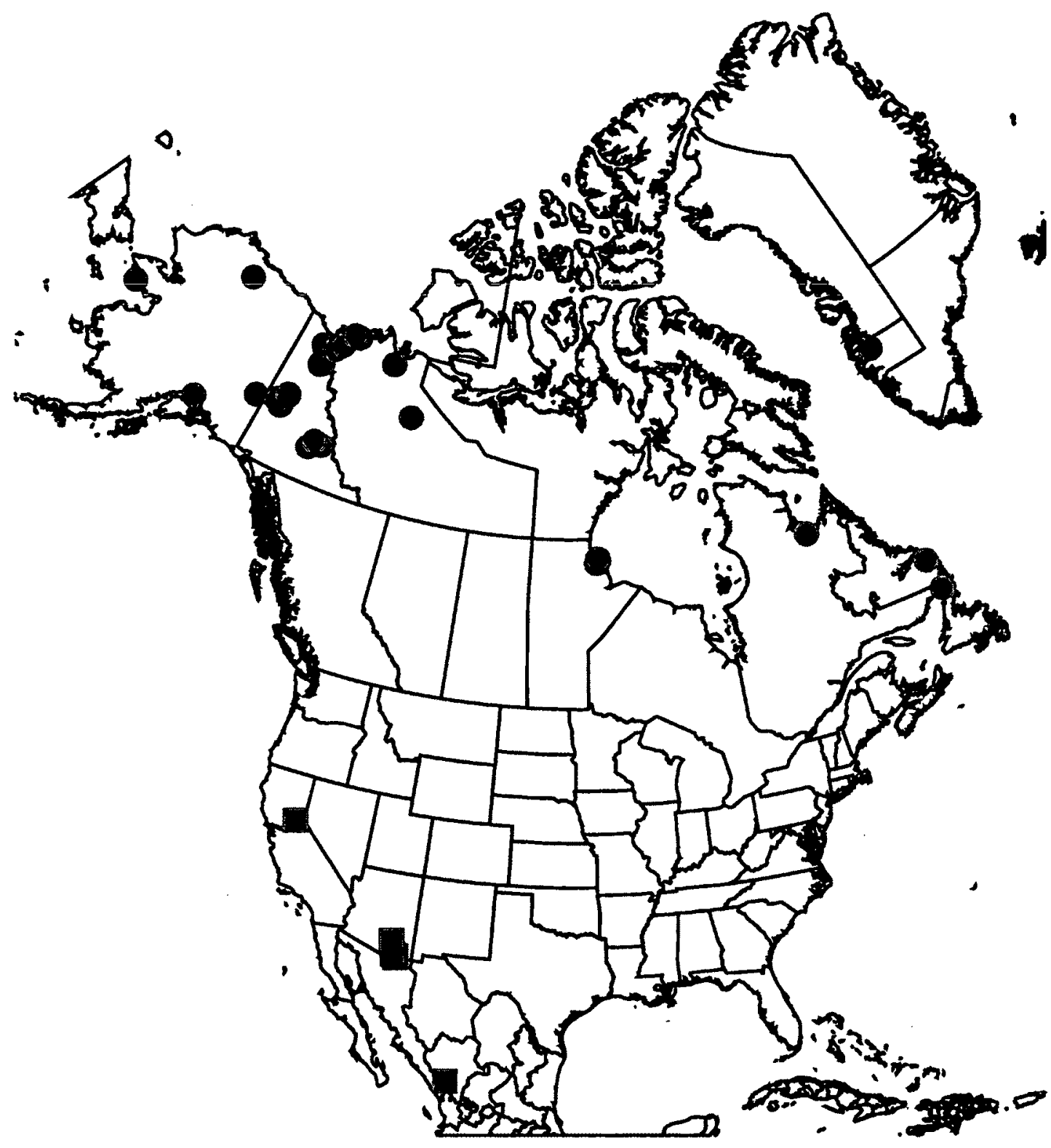

Fig. 22: Dasysyrphus lotus (squares) and D. nigricornis (circles) range map (symbols indicate specimen point dataj. 


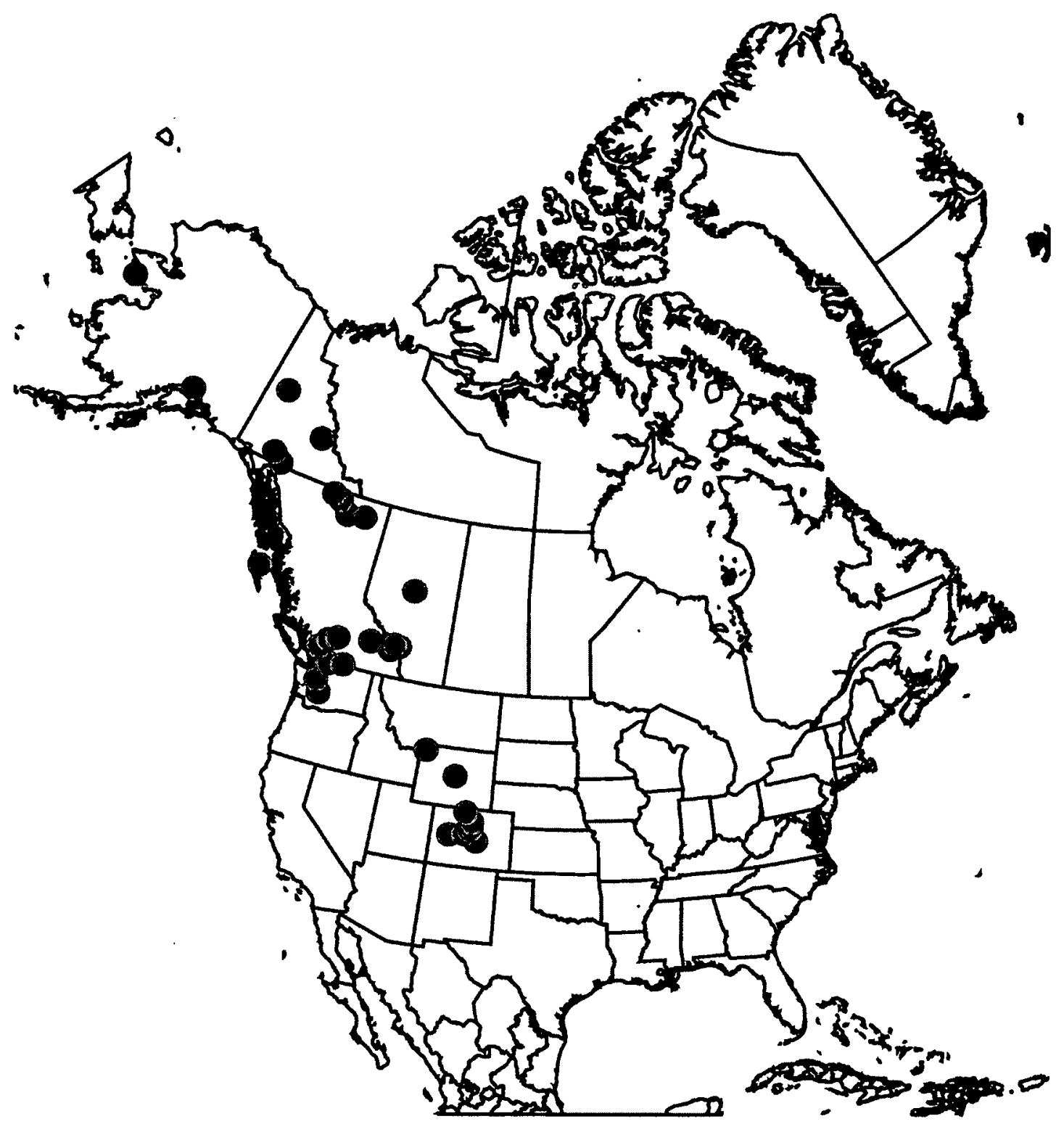

Fig. 23: Dasysyrphus occidualis range map (circles indicate specimen point data). 


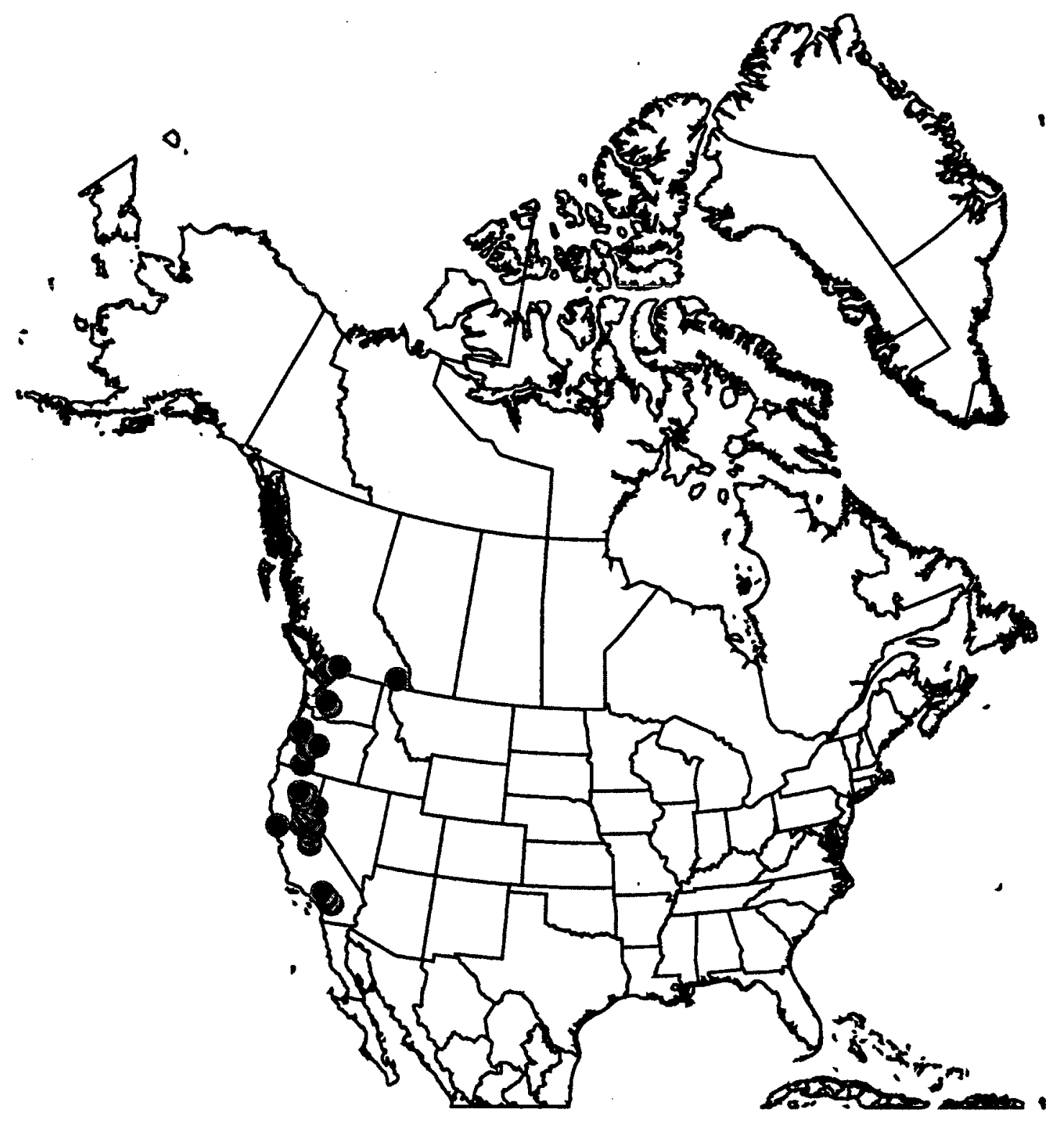

Fig. 24: Dasysyrphus pacificus range map (circles indicate specimen point data). 


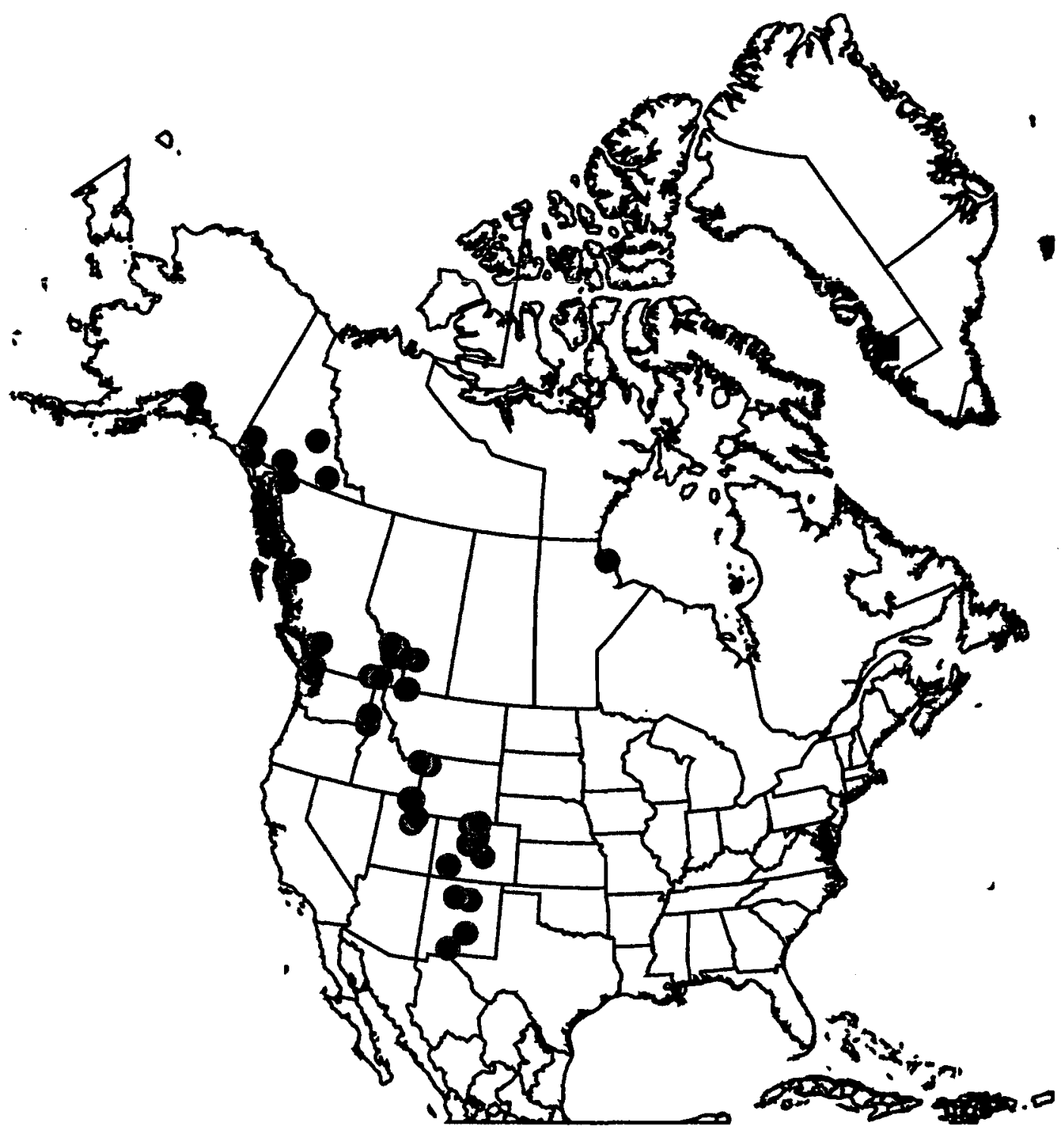

Fig. 25: Dasysyrphus pauxillus (circles) and D. pinastri (squares) range map (symbols indicate specimen point data). 


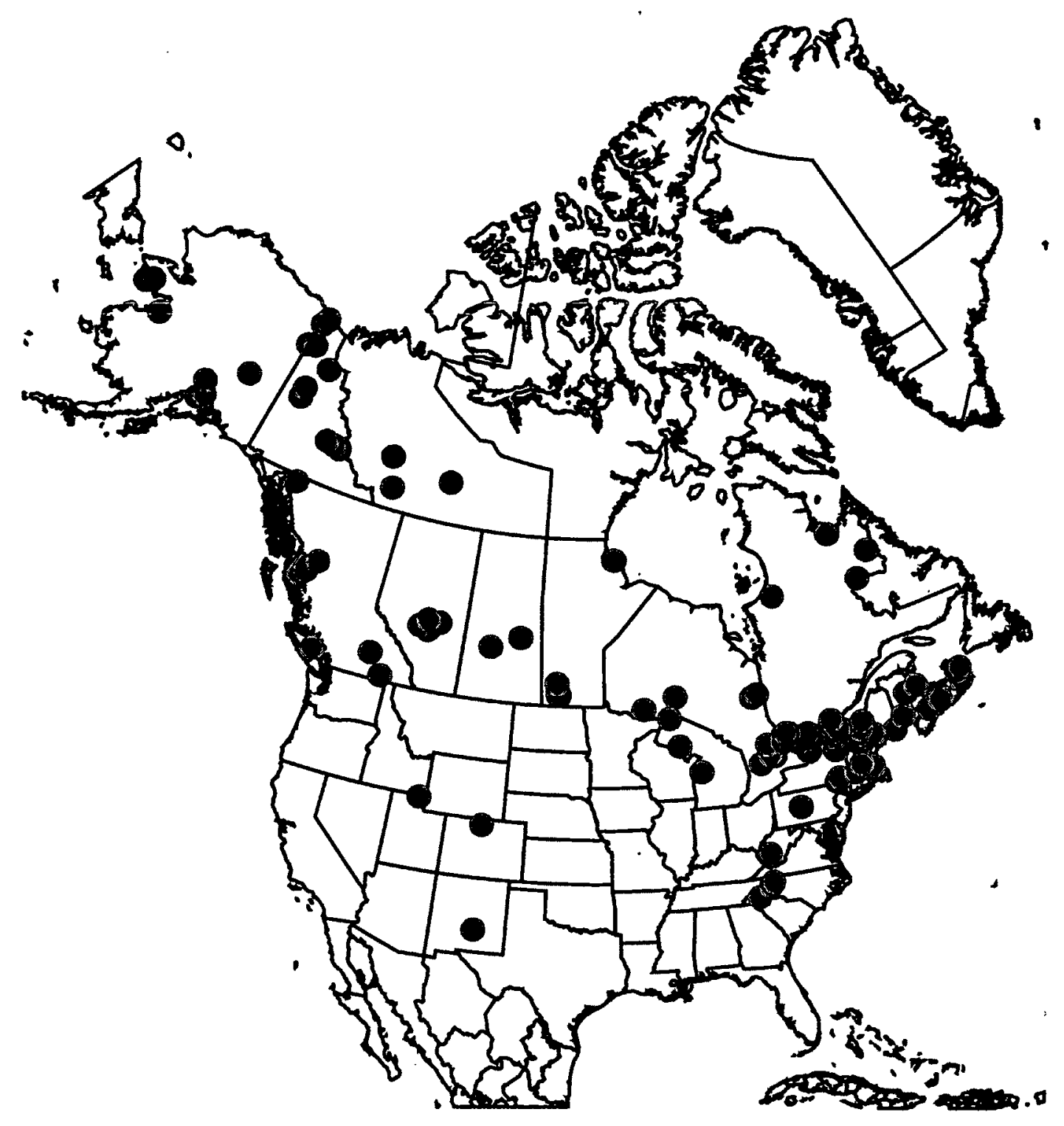

Fig 26: Dasysyrphus venustus range map (circles indicate specimen point data). 